\title{
A GENERIC-LEVEL REVISION OF THE SPIDER SUBFAMILY COELOTINAE (ARANEAE, AMAUROBIIDAE)
}

\section{XIN-PING WANG}

Department of Invertebrate Zoology, American Museum of Natural History; Current address: Department of Entomology, California Academy of Sciences, Golden Gate Park, San Francisco, CA 94118

BULLETIN OF THE AMERICAN MUSEUM OF NATURAL HISTORY CENTRAL PARK WEST AT 79TH STREET, NEW YORK, NY 10024

Number 269, 150 pp., 448 figures

Issued May 30, 2002 


\section{CONTENTS}

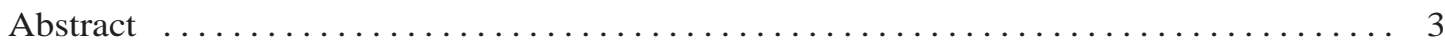



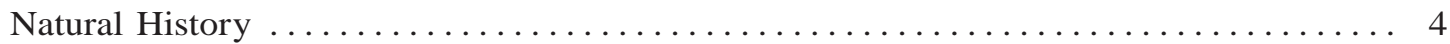

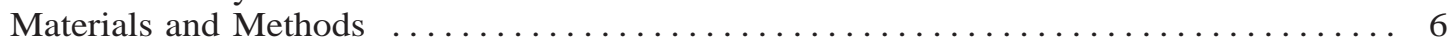

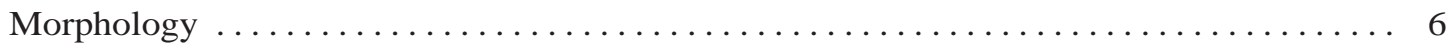

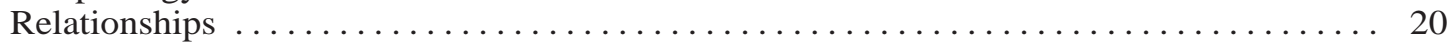

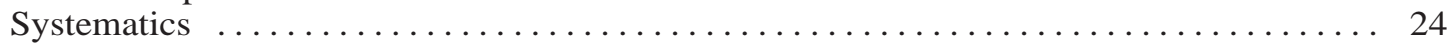

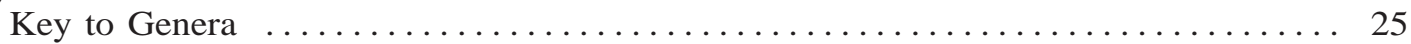

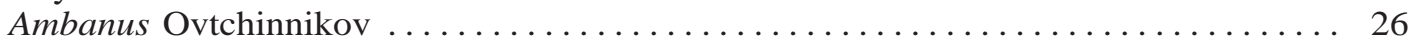



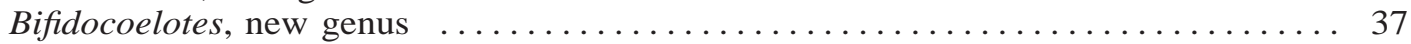

Coelotes Blackwall ................................... 40

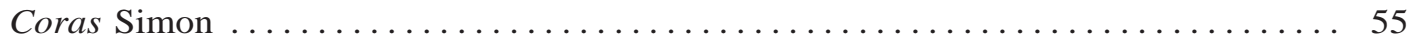

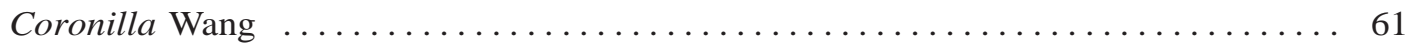

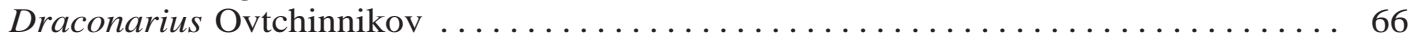

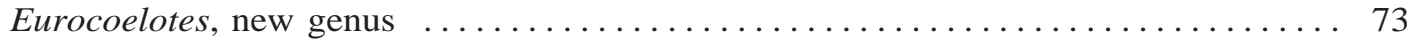

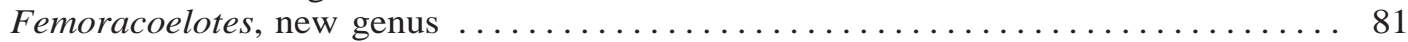

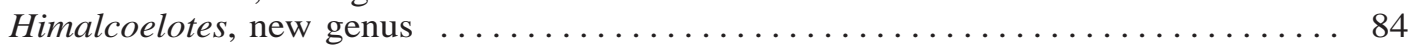

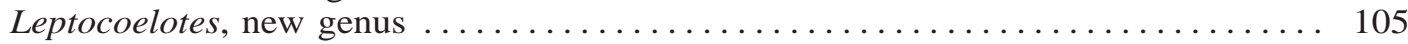

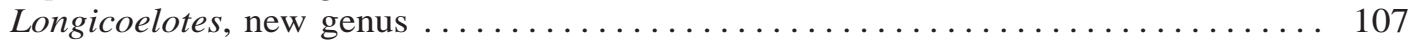



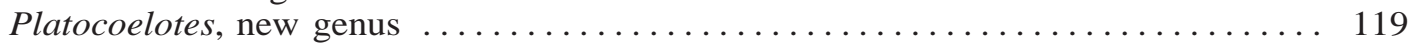

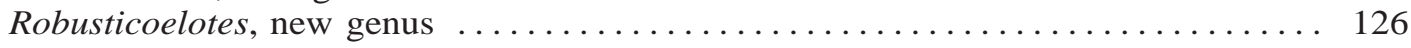

Spiricoelotes, new genus ................................ 129

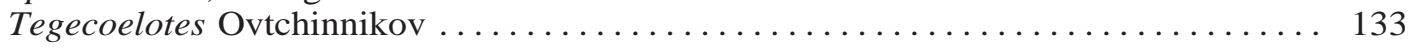

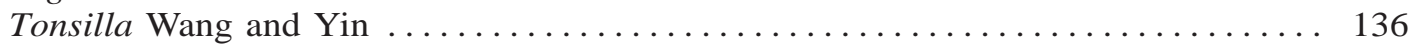





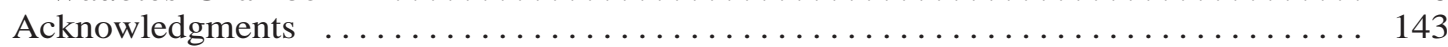

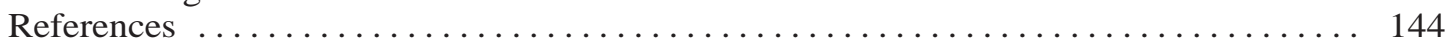

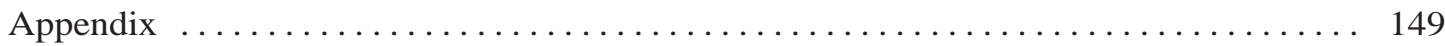




\begin{abstract}
The Holarctic coelotine spiders are revised at the generic level. Coelotine morphology, particularly genitalic morphology, is described, the subfamily and generic limits are defined, and a cladistic hypothesis of generic relationships is presented.

The subfamily Coelotinae is defined to include 20 genera and 277 species. The 20 genera are: Ambanus Ovtchinnikov, 1999, with 18 species from far eastern Russia, northeastern China, Japan, and Korea (including 6 new combinations); Asiacoelotes, new genus, with 15 East Asian species (all new combinations, with 3 new synonyms); Bifidocoelotes, new genus, with 2 species from China (both new combinations); Coelotes Blackwall, 1841, with 123 species from Europe and Asia (including 2 new synonyms); Coras Simon, 1898, with 15 North American species and 2 species from China; Coronilla Wang, 1994, with 2 species from China (including 2 new synonyms); Draconarius Ovtchinnikov, 1999, with 26 Asian species (including 25 new combinations and 1 new synonym); Eurocoelotes, new genus, with 11 European species (all new combinations); Femoracoelotes, new genus, with 2 species from Taiwan (both new combinations); Himalcoelotes, new genus, fully revised here, with 10 species from the Himalayas (including 2 new combinations and 8 new species); Leptocoelotes, new genus, with 2 species from China (both new combinations); Longicoelotes, new genus, with 1 new species from China; Paracoelotes Brignoli, 1982, with 16 European and Asian species (including 5 new combinations and 5 new synonyms); Platocoelotes, new genus, with 4 species from China (all new combinations); Robusticoelotes, new genus, with 1 newly combined species from China; Spiricoelotes, new genus, with 2 East Asian species (both new combinations); Tegecoelotes Ovtchinnikov, 1999, with 5 species from far eastern Russia, northeastern China, Japan, and Korea (including 4 new combinations); Tonsilla Wang and Yin, 1992, with 3 species from China; Urocoras Ovtchinnikov, 1999, with 5 species from East Europe (including 2 new combinations); and Wadotes Chamberlin, 1925, with 11 species from North American and 1 from China.
\end{abstract}

\section{INTRODUCTION}

The first known coelotine, Paracoelotes segestriformis (Dufour, 1820), was described from France as Drassus. Blackwall (1841) described the new genus Coelotes, in the Agelenidae, for the species Clubiona saxatilis Blackwall, 1833, which was later placed as a junior synonym of Coelotes atropos (Walckenaer, 1830). Several other coelotines were described before 1842 but were not placed in the genus Coelotes: Drassus trucidator Walckenaer, 1830, and Clubiona tigrina Walckenaer, 1842 (both are junior synonyms of C. atropos); Amaurobius subterraneus C. L. Koch, 1837, and Amaurobius tigrinus C. L. Koch, 1837 (both are junior synonyms of C. terrestris); Amaurobius roscidus C. L. Koch, 1834 (a junior synonym of $P$. segestriformis); and Tegenaria nemorensis Blackwall, 1841 (a junior synonym of Coras medicinalis Hentz, 1821). From 1855 to 1898 , a series of about 43 new species of coelotines were described from Europe (Koch, 1855, 1868, 1878; Kroneberg, 1875; Simon, 1870, 1875, 1876, 1880b, 1891; O. P.-Cambridge, 1873; Kulczyn'ski, 1887,
1898; Chyzer and Kulczyn'ski, 1897), Asia (Simon, 1880a; Karsch, 1879, 1881; O. P.Cambridge, 1885), and North America (Simon, 1898; Keyserling, 1881, 1887; Emerton, 1889; Banks, 1892, 1895, 1898;). F.O.P.Cambridge (1893) proposed the subfamily Coelotinae, in the family Agelenidae, to include the genus Coelotes. Simon (1898) proposed the genus Coras with the North American coelotine, $C$. medicinalis, as type species.

During the 1900s, more than 250 new coelotines were described, mostly in the genus Coelotes; however, some were put in the genera Amaurobius (Kulczyn'ski, 1906; Drensky, 1915; Schenkel, 1925), Tegenaria (Bösenberg and Strand, 1906), and Agelena (Bösenberg and Strand, 1906; Charitonov, 1969). In 1925, Chamberlin proposed a third coelotine genus, Wadotes, that is distributed in the eastern part of North America. It was revised by Muma (1947) and Bennett (1987), with 11 species recognized. A fourth coelotine genus, Paracoelotes, was proposed by Brignoli (1982), with the type species Coelotes armeniacus Brignoli, 1978, from Tur- 


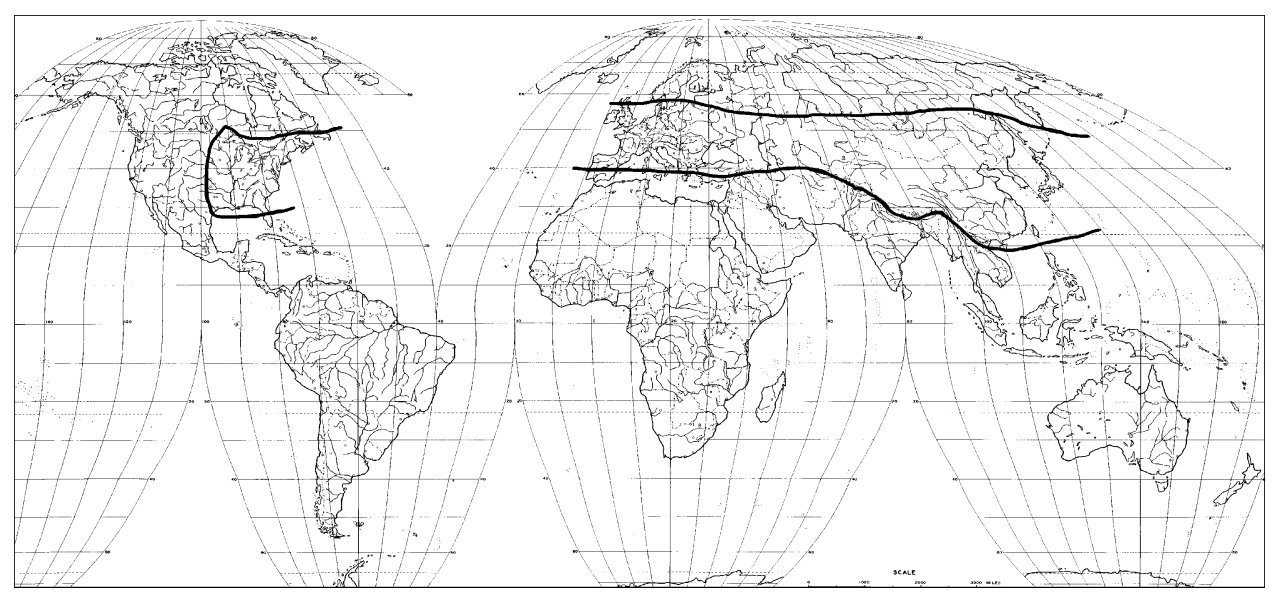

Fig. 1. Records of Coelotinae.

key, to accommodate some 10 European and Asian species. The genera Tonsilla Wang and Yin, 1992, and Coronilla Wang, 1994, were established with 3 and 2 species, respectively, from China. In 1999, Ovtchinnikov recognized four new genera: Ambanus Ovtchinnikov, 1999, from northern China, Korea, and far eastern Russia, with 11 species; Draconarius Ovtchinnikov, 1999, from Middle Asia, with only 1 species; Tegecoelotes Ovtchinnikov, 1999, from northeastern China, far eastern Russia, Japan, and Korea, with 5 species described; and Urocoras Ovtchinnikov, 1999, from eastern Europe, with 3 species included.

The most comprehensive species-level revisions of Palearctic coelotines were those of de Blauwe (1973) and Deltshev (1990), who revised coelotines from the Mediterranean region and Bulgaria, respectively. Coelotines from other Palearctic regions have been treated only in isolation. Many species are known only from one sex, and many may be synonyms. The genera included in Coelotinae have never been revised. Although Lehtinen (1967) and Brignoli (1982) exposed problems existing in Coelotinae taxonomy, thus far no work has been done on the world genera and species.

In addition to problems existing in defining and recognizing coelotine genera and species, the phylogenetic relationships of the group in relation to other taxa were not resolved. F.O.P.-Cambridge's subfamily Coelotinae was not accepted by Simon (1898) and Roewer (1954). Lehtinen (1967) was the first to list Coelotes, Coras, and Wadotes in Coelotinae, although he pointed out that coelotines strongly resemble the cribellate amaurobiid genera Amaurobius C. L. Koch, 1837, Tamgrinia Lehtinen, 1967, and Taira Lehtinen, 1967, in general appearance and also in habits (i.e., sheet web is reduced to a mere dwelling tube). Wunderlich (1986) synonymized Amaurobiidae and Agelenidae. Unfortunately, no evidence for this was given in his paper. Platnick (1989) did not accept the synonymy of Agelenidae and Amaurobiidae, but he (Platnick, 1989, 1993, 1997) followed Wunderlich in considering Coelotes and allied genera to be more closely related to Amaurobius than to Agelena, and therefore listed coelotines under the family Amaurobiidae.

In this paper, 20 valid coelotine genera are recognized, of which 10 are newly described. The genera are diagnosed, composition and distribution information is provided, and the type species are described. The relationships among coelotine genera are analyzed.

\section{NATURAL HISTORY}

The Holarctic spider subfamily Coelotinae is one of the most common spider taxa in North America west to the Rocky Mountains and north to southern Canada, in Europe north to southern Sweden and Finland, and in Asia north to far eastern Russia and south to Nepal and northern Vietnam (fig. 1). Coe- 


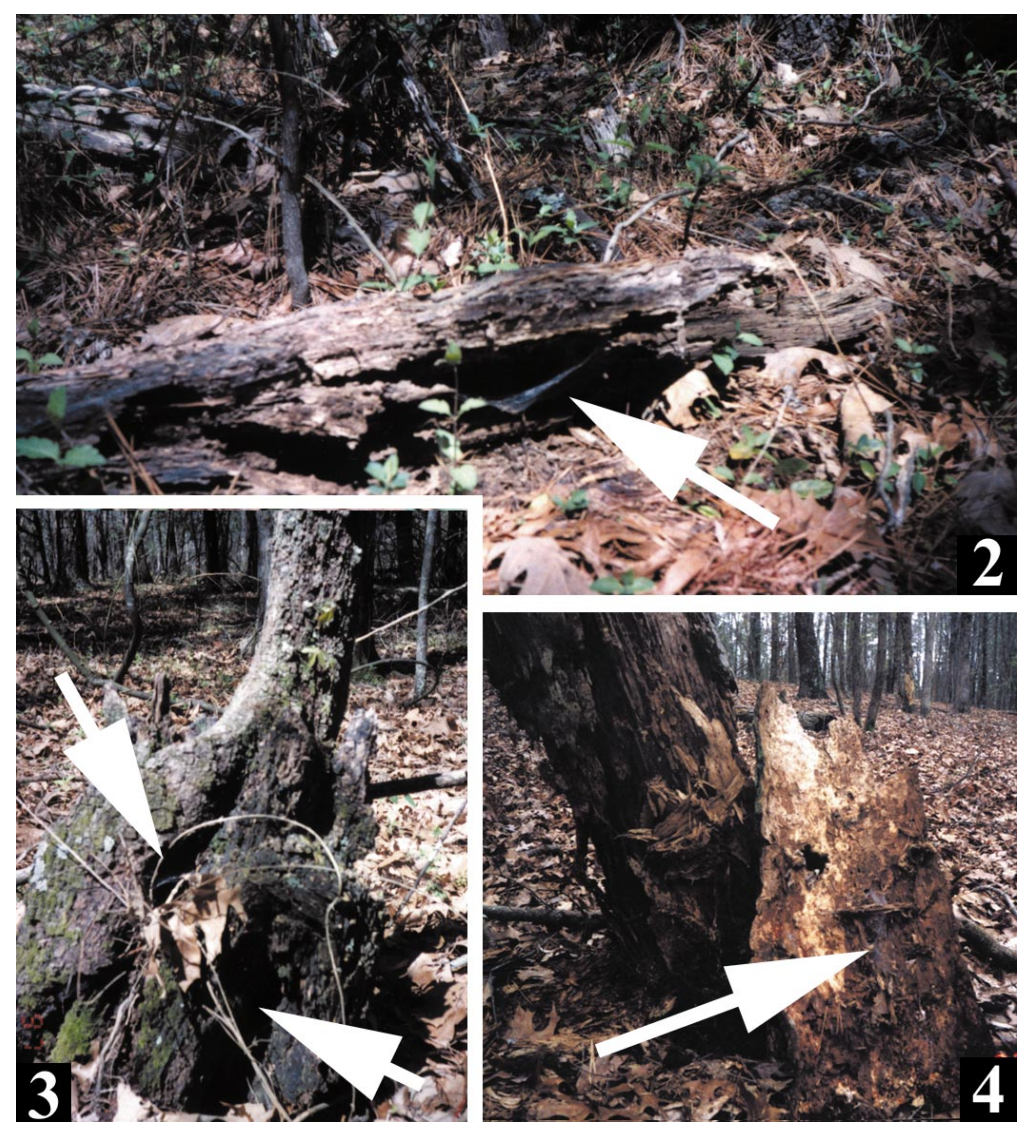

Figs. 2-4. Habitats of Coras spp., from Mississippi. 2. Under dead log. 3. Inside tree holes. 4. Under tree bark.

lotines occur from sea level to as high as $4500 \mathrm{~m}$ in the Himalayas, and are found on cliffs, under logs, stones, bark, leaf litter, and other objects, in corners, holes, and crevices of buildings, and in caves. Some species, such as Coelotes terrestris, can be found in densities of as much as 1.2 webs per square meter in an ideal microclimate, such as in hedges with the ground covered by litter (Petto, 1990). All species appear to build small funnel webs with several retreats beneath objects, usually deep into tree holes, rock crevices, walls, or ground slopes (figs. 2-4).

Female coelotines make oval egg sacs attached to their webs. The egg sacs of Coras species can be collected either in late fall or in spring. A female of Coras juvenilis (Keyserling, 1881), collected in October, made its egg sacs in late October and November in the lab oratory, and 82 young were hatched in a single egg sac. Numerous females of Coras lamellosus (Keyserling, 1887) were collected together with egg sacs in March from Mississippi. A female of Coras medicinalis (Hertz, 1821) was collected in April from Connecticut, made its egg sac in May with about 75 eggs inside, and died in July. Another female Coras medicinalis, collected in June from Connecticut, made 4 egg sacs, and it died the following January.

Coelotine life history is poorly known. Catley (1992) found that Coelotes atropos is winter-active, and juveniles were capable of silk production at $-5^{\circ} \mathrm{C}$. The biology of $\mathrm{Coe}$ lotes terrestris (Wider, 1834) was studied by Tretzel (1961; from Petto, 1990): "The lifecycle of the females is biennial and of the male annual. After mating in the autumn most males die. Females hibernate and then 
build one or two egg-cocoons in May or June of the following year. Spiderlings hatch about four weeks later, stay in their mother's web for some weeks and feed on her prey during this time." Based on collections examined and fieldwork, adults may be found throughout the year. In North America, many adult males and females may survive from September to the following spring. In Europe and Middle Asia, mass occurrences of adult coelotines seem to begin as early as July and last to late fall. Published collection dates of coelotines from China show that adult males and females occur in large numbers in September in the north and in late October in the south. An observation of pitfall collections (Wang et al., 2001) shows that adult coelotines in central Taiwan may occur year-round but are most abundant from October to January.

The prey of coelotines includes active arthropods captured on the sheet web (Tretzel, 1961). The prey composition of $C$. terrestris in habitat such as hedges with ground litter included more than $60 \%$ beetles and about 20\% Diptera (Petto, 1990).

\section{MATERIALS AND METHODS}

The morphology, including somatic and genitalic characters, of Coelotes atropos (Walckenaer, 1830) is described in detail. The type species of each genus (except for that of Draconarius, which was not available) is described based on available type specimens. Due to similarities in coelotine somatic morphology, descriptions of the type species of each genus use only variable informative structures, such as eyes, chelicerae, spinnerets, and male and female genitalic structures, with special emphasis on genitalic structures in redescriptions of existing species. Eyes, legs, and body lengths are given in the new species descriptions.

All measurements are in millimeters. Leg measurements are shown as: total length (femur, patella + tibia, metatarsus, tarsus). All scale lines are $0.2 \mathrm{~mm}$ long except where indicated otherwise. Eye sizes are measured as the maximum diameter from either above or in front. Leg spines are described for the dorsal $(\mathrm{d})$, prolateral $(\mathrm{p})$, ventral $(\mathrm{v})$, and retrolateral (r) surfaces.
Tracheae were examined by first dissecting off the dorsal cuticle of the abdomen, boiling in about $10 \% \mathrm{KOH}$ for a few minutes, and then cleaning carefully in water. Male palps were expanded by first being immersed in about $10 \% \mathrm{KOH}$ for about 24 hours, and then transferred to water for complete expansion. All materials observed under SEM were either critical point-dried or air-dried and then sputter-coated.

\section{AbBreviations}

$\begin{array}{ll}\text { AC } & \text { aciniform gland spigots } \\ \text { ALE } & \text { anterior lateral eyes } \\ \text { ALS } & \text { anterior lateral spinneret } \\ \text { AME } & \text { anterior median eyes } \\ \text { C } & \text { conductor } \\ \text { CD } & \text { copulatory duct } \\ \text { CDd } & \text { conductor dorsal apophysis } \\ \text { CL } & \text { conductor lamella } \\ \text { CY } & \text { cylindrical gland spigots } \\ \text { EM } & \text { embolus } \\ \text { EMb } & \text { embolic base } \\ \text { MA } & \text { median apophysis } \\ \text { mAP } & \text { minor ampullate gland spigots } \\ \text { MAP } & \text { major ampullate gland spigots } \\ \text { PI } & \text { piriform gland spigots } \\ \text { PLE } & \text { posterior lateral eyes } \\ \text { PLS } & \text { posterior lateral spinneret } \\ \text { PME } & \text { posterior median eyes } \\ \text { PMS } & \text { posterior median spinneret } \\ \text { RTA } & \text { retrolateral tibial apophysis } \\ \text { S } & \text { spermathecae } \\ \text { SH } & \text { spermathecal head } \\ \text { ST } & \text { subtegulum } \\ \text { T } & \text { tegulum } \\ \text { TS } & \text { tegulum sclerite } \\ & \end{array}$

\section{MORPHOLOGY}

\section{Coelotes atropos (Walckenaer)}

No coelotine has ever been described in detail. A representative species, Coelotes atropos, which is a relatively common European species and the type species of the genus Coelotes, was chosen here for detailed description.

Body form (fig. 5): Ecribellate spiders. Total length about 9.00-12.0 (female) and 8.00-9.50 (male). Carapace elongate, reddish brown, moderately narrowed in ocular area, sparsely covered with short, long black setae; few strong setae on clypeus, ocular area, and middle carapace; longitudinal fovea moder- 


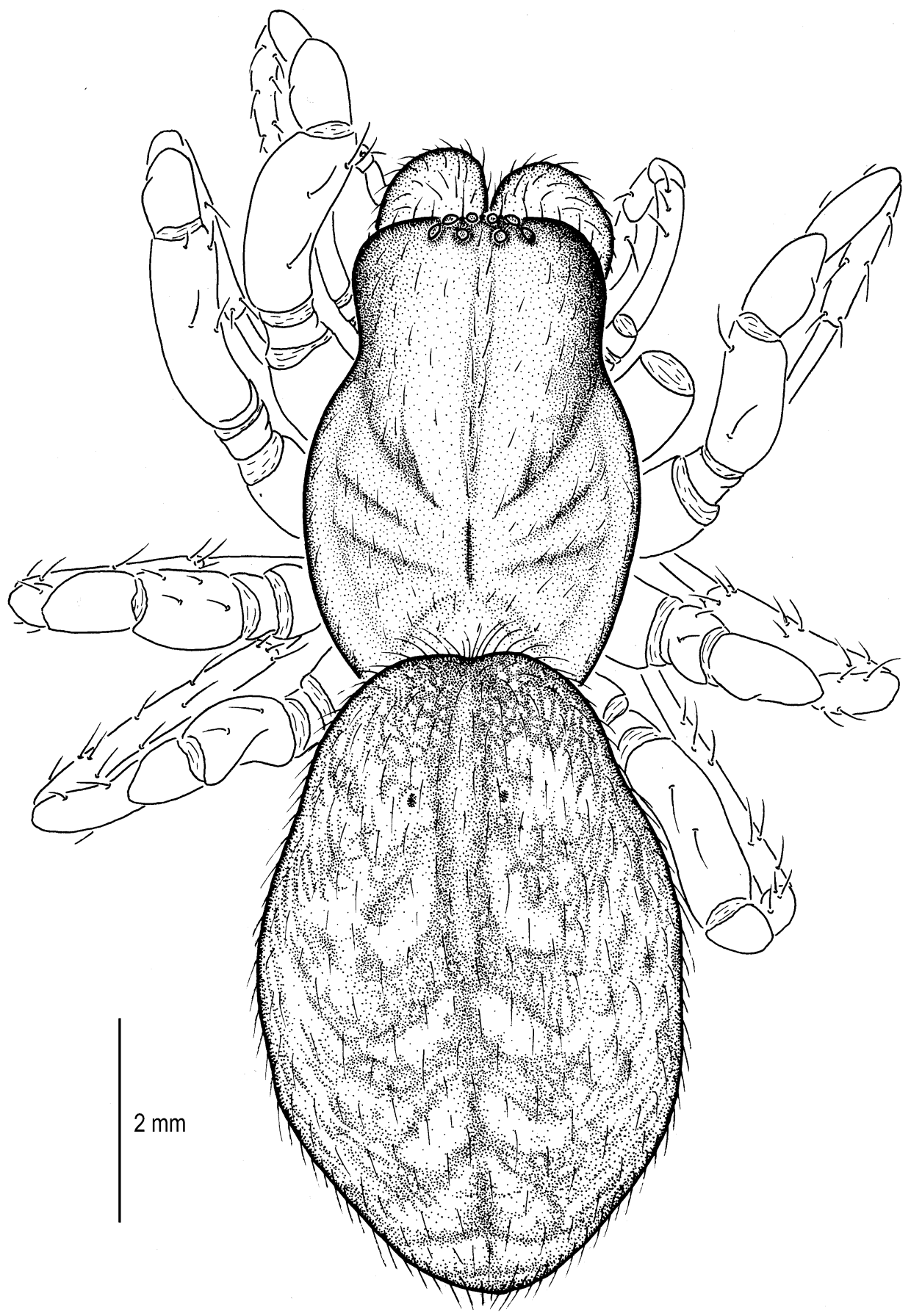

Fig. 5. Coelotes atropos (Walckenaer), female body, dorsal view. 
ately depressed. Legs moderately long, strong. Abdomen dark brown, with dark maculation, heavily covered with short setae. Spinnerets moderately elongated.

Eyes (fig. 6): From front, anterior eye row slightly procurved, posterior row procurved; eye sizes and arrangements: AME smaller than ALE, ALE slightly larger than PLE, PME subequal to PLE, slightly larger than AME; AME may be separated by their diameter or slightly less than their diameter, AME-ALE slightly shorter than AME-AME, PME separated by their diameter, PME-PLE widely separated by 1.5-2.0 PLE diameter, lateral eyes close together; AME-PME separated by PME diameter.

Clypeus (fig. 6): Height two times AME diameter, covered with long, strong setae; chilum divided, hairless.

Chelicerae (figs. 6-8, 12-19): Three promarginal teeth, with middle one largest, and three retromarginal teeth, subequal in size (fig. 8); condyle large (figs. 7, 8); cheliceral glands open distally near base of fang on both dorsal and ventral sides, gland openings situated inside striated grooves (figs. 13, 14); dorsal chelicerae covered with long setae, with strongly elevated base (figs. 6, 7); chelicerae ventrally flat, with proximal short setae and inner long, fine setae (figs. 8, 12, 15, 16); fangs moderately long (figs. 8, 18, 19); venom gland large, reaching middle of thorax; anterior face of chelicerae covered with dense, long, strong setae (figs. 8, 17).

Endites (fig. 9): Rectangular, with anterior scopula and linear serrula.

Labium (fig. 9): Rectangular, slightly longer than wide, slightly notched distally.

Sternum (fig. 11): Shield-shaped, sparsely covered with long dark setae (setae not in figure), heavily sclerotized.

Legs (figs. 20-30): Legs medium to long. I, IV longest, almost subequal, leg III shortest; length of 1st leg patella+tibia shorter than carapace length; trochanters not notched or with very weak notch; tibiae with about four rows of trichobothria; metatarsi and tarsi with one row of trichobothria, their length gradually increasing distally; trichobothria with large hoods transversely striated, small hoods smooth. Tarsal organ situated close to distal end of tarsus, slightly anterior of most distal trichobothrium. Tarsi with three claws, superior tarsal claws with 10-12 teeth, inferior tarsal claws usually with 2-3 teeth; scopulae absent; leg spination often varies among individuals, typical leg spination pattern: femur: I p0-0-2, d1-1-1(0); II p0-0-1, r0-02 , d1-1-1(0); III p0-1-2, r0-0-1, d1-1-0; IV p0-0-1, d1-1-0; patella: p1(0), r1(0), $\mathrm{d} 1(0)$; tibia: I p0-0-0(1), r0-0-2, v2-2-2; II p1-1-0, v2-2-2; III, IV p1-1-0, r1-1-0, v22-2; metatarsus: I p0-0-0(1), v2-2-2; II p01-2, v2-2-2; III p1-2-2, r1-2-2, v2-2-2; IV p1(2)-2-2, r1(2)-2-2, v2-2-2; tarsus: III, IV p1-1-0, r1-1-0.

Trachea (fig. 31): Simple, limited to abdomen; median spiracle situated close to spinnerets and connected to relatively narrow atrium from which two lateral and two median tubes arise.

Spinnerets (figs. 32-37): Colulus absent; ALS cylindrical, apex of ALS with 2 major ampullate gland spigots (MAP) at mesal margins, 15-30 piriform gland spigots; PMS with spigots situated on distal half of segment, both sexes PMS with 1 or 2 minor ampullate gland spigots (mAP), 30-50 aciniform gland spigots (AC), female PMS with 2 cylindrical gland spigots (CY); PLS with second segment about same length as first segment, with 30-40 aciniform gland spigots in both sexes, and 2 cylindrical gland spigots in female.

Female genitalia (figs. 38-43): Epigynal teeth short, situated on lateral atrium; atrium reduced to atrial slits; copulatory ducts short; spermathecal heads short, broad, situated anteriorly; spermathecae longitudinally elongated with indistinct stalks and bases.

Male genitalia (figs. 44-54): Palpal femur long, with three strong spines dorsally, femoral apophysis absent; patella short, with long, strong dorsal spine; patellar apophysis broad; tibia short, almost same length as patella, with three conspicuous strong spines on prolateral side; retrolateral tibial apophysis (RTA) extended along tibia length, with distal end slightly extended beyond tibia; a small apophysis, called lateral tibial apophysis here, arises from tibia retrolaterally on dorsal side of RTA, and differs both in morphology and position with the dorsal tibial apophysis in other taxa, such as some phyxelidine species (Griswold, 1990) and amaurobiid species (Leech, 1972); cymbium long, 



Figs. 6-11. Coelotes atropos (Walckenaer), female. 6. Eyes and chelicerae, front view. 7. Chelicera, lateral view. 8. Chelicera, ventral view. 9. Labium, endite, and palp, ventral view. 10. Palp, dorsal view. 11. Sternum. 


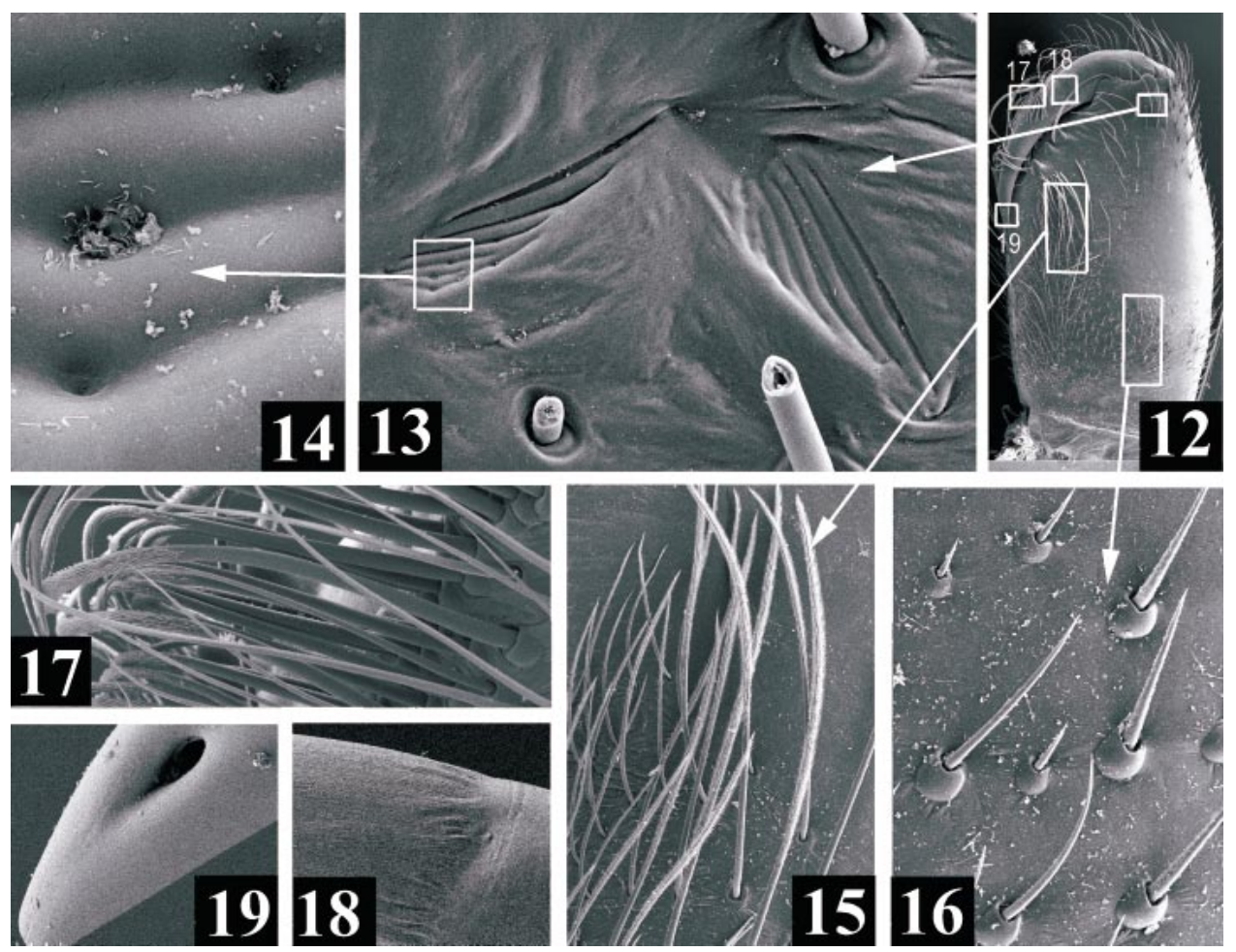

Figs. 12-19. Coelotes atropos (Walckenaer), female. 12. Chelicera, ventral view. 13. Cheliceral glands. 14. Cheliceral gland, close view. 15. Inner, distal long setae of chelicera. 16. Proximal short setae of chelicera. 17. Promargin setae of chelicera. 18. Median depression of cheliceral fang. 19. Apex of cheliceral fang.

strongly extended anteriorly beyond alveolus, with many conspicuous, long spines on distal and prolateral surfaces; cymbial furrow short, less than half cymbial length, with dorsal edge slightly concave; a rectangular sclerotization of the distal tegular surface, called tegular sclerite here, is present and unique for coelotines; treated tegular sclerite as radix (Bennett, 1987) was not appropriate because radix usually refers to the proximal segment connecting the embolus to the tegulum (Gering, 1953; Comstock, 1910); conductor short; an apophysis arises dorsally on the conductor, which has been described as terminal apophysis (Bennett, 1987), is here called the conductor dorsal apophysis; terminal apophysis often related to embolus rather than to conductor (Comstock, 1910; Sierwald, 1990); conductor dorsal apophysis broad, with rough surface; conductor lamella moderately developed; embolus moderately long, prolateral in origin; median apophysis spoonlike, short, rounded, with smooth distal end.

\section{Coelotinae General Morphology}

Within coelotines, both male and female somatic structures are relatively uniform; this condition offers few characters for identification and phylogenetic analysis of species and genera. However, a few characters vary within coelotines, as discussed here.

Eyes: Coelotines often have AME smaller than ALE. AME are much smaller than ALE (e.g., Bifidocoelotes, fig. 90) or slightly smaller (e.g., Himalcoelotes martensi, fig. 270). In Coras, AME are larger than ALE (fig. 147).

Cheliceral teeth: In most coelotines, the promargins of the chelicerae are relatively constant with three teeth, but in Leptocoelotes, Robusticoelotes, and Spiricoelotes, there are more than four teeth (figs. 301, 356, 365). 


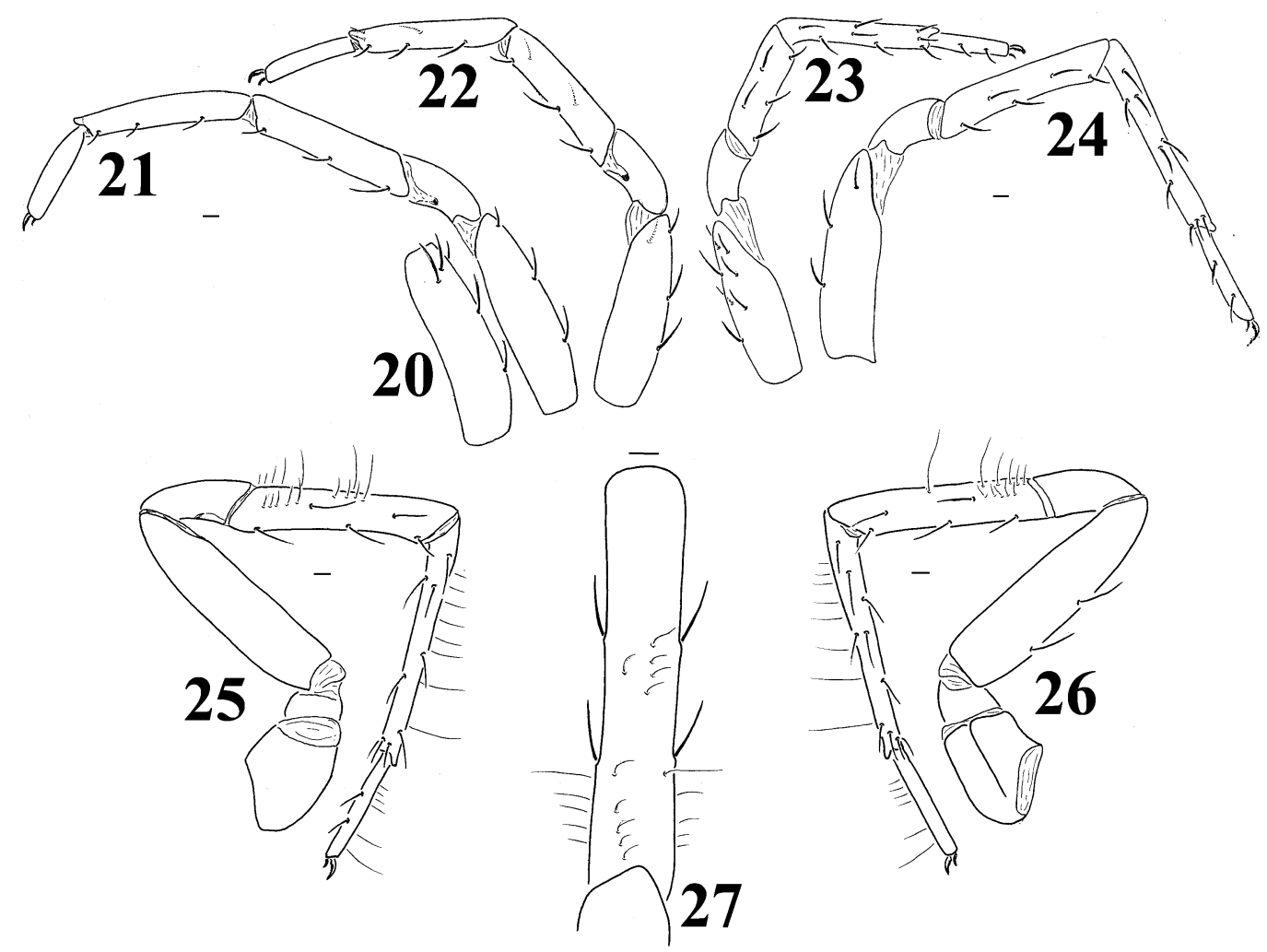

Figs. 20-27. Coelotes atropos (Walckenaer), female, legs. 20. First leg femur, right. 21-24. First to fourth legs, left. 25, 26. Fourth leg, showing trichobothria distribution. 27. Fourth leg tibia, showing trichobothria distribution.

The cheliceral retromargin varies from two (e.g., Ambanus and Wadotes, figs. 63, 418) to three (e.g., Coelotes and Paracoelotes, figs. 8, 329), four (e.g., Coronilla and Leptocoelotes, figs. 170, 301), or even more than five teeth (e.g., Robusticoelotes and Spiricoelotes, figs. 356, 365).

Trichobothria and tarsal organ: The distribution of trichobothria in coelotines is like that of $C$. atropos. The tarsal organ is usually situated close to the distal end of tarsus and anterior to the most distal trichobothrium (as in most coelotines, figs. 28, 172, 391). However, there are variations. In examined species of Draconarius, the tarsal organ is situated far from the distal end and close to the second most distal trichobothrium or even farther proximally (figs. 186, 202); in $\mathrm{Fe}$ moracoelotes (figs. 234, 235), the tarsal organ is near the third most distal trichobothrium; in Leptocoelotes, it is near distalmost trichobothrium (figs. 305, 306). The tarsal organ was not seen in examined specimens of Platocoelotes (figs. 343, 345).

Spinnerets: The types of spigots are constant in coelotines, but the number varies among genera and species and between sexes: the number of piriform gland spigots on the ALS varies from about 15 to 110 , and the number of aciniform gland spigots on the PMS and PLS also varies from 20 to 120; both the PMS and PLS usually have 2 cylindrical gland spigots, but Paracoelotes has 3 or 4 cylindrical gland spigots on PMS (fig. 334), and 3 cylindrical gland spigots were seen on Ambanus PLS (fig. 66).

\section{Coelotinae Genitalic Morphology}

Female

In Platocoelotes, the sclerotized epigynum is strongly enlarged to occupy most of the 

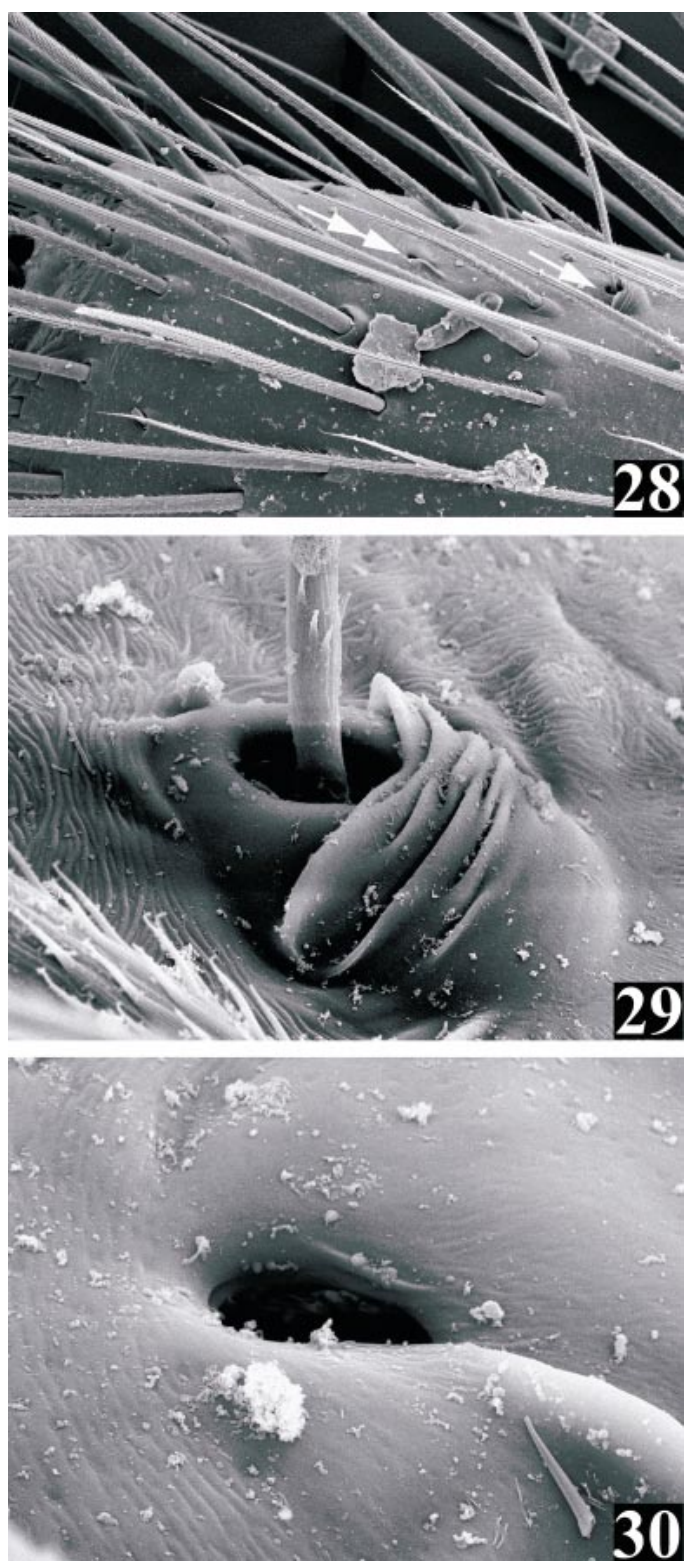

Figs. 28-30. Coelotes atropos (Walckenaer). 28. Tarsus, showing position of tarsal organ (double arrows) and the distalmost trichobothrium (single arrow). 29. Trichobothrium. 30. Tarsal organ.

front epigastric furrow (fig. 336). In Coronilla, the posterior margin of the epigynum is strongly expanded posteriorly (figs. 158, 159). The epigynum is characterized by the atrium, epigynal teeth, and epigynal hoods.

Atrium: The atrium here refers to the con- cavity on the epigynum plate which leads to the copulatory ducts of the vulva. It may be large (e.g., Ambanus and Paracoelotes, figs. 57,323 ) or merely an atrial slit (e.g., $W a$ dotes and Asiacoelotes, figs. 410, 70). In most coelotines, the atrium is situated medially or more or less anteriorly on the epigynal plate, but in Coelotes rufulus (Wang et al., 1990), the atrium originates posteriorly on the tonguelike posterior extension of the epigynum. The anterior atrial margin may be strongly extended posteriorly, as in Tonsilla and Paracoelotes (figs. 384, 323). In Coronilla, the middle part of the atrium may form a transverse carina (figs. 158, 159). In most coelotines, the lateral atrial margins are more or less parallel and widely separated, whereas in Coras, the lateral atrial margins are more widely separated anteriorly than posteriorly and enclose a vase-shaped area (figs. 137, 139, 141, 142).

Epigynal teeth: These are present in most coelotines but absent in some (e.g., Coronilla and Longicoelotes, figs. 158, 310). There may be a single, medially situated epigynal tooth, as in Bifidocoelotes and Wadotes (figs. 86,410 ), or teeth may be paired (most coelotines). If present, it or they may be extremely long (e.g., Himalcoelotes and Urocoras, figs. 265, 400), broad (e.g., Leptocoelotes and Tegecoelotes, figs. 296, 375), or slender. In Asiacoelotes, epigynal teeth occur anteriorly on epigynal plate (fig. 70), whereas in Tonsilla, epigynal teeth are located close together on the anterior atrial margin (fig. 384).

Epigynal hoods: These are a pair of small, sclerotized, hairless plates that are usually situated laterally on the epigynal plate (e.g., Ambanus, fig. 57). In Platocoelotes (fig. 336), however, the hoods are located posteriorly and close to the epigastric furrow. A pair of large muscles, which are attached inside the hoods, may be important in female genitalic movement during copulation. The hoods can be either strongly depressed, as in Spiricoelotes (figs. 360, 362), or only moderately depressed.

Fertilization ducts: These are very small in all coelotines and arise from the posterior end of the spermathecae.

Copulatory ducts: These are usually membranous and may be large and broad (e.g., 

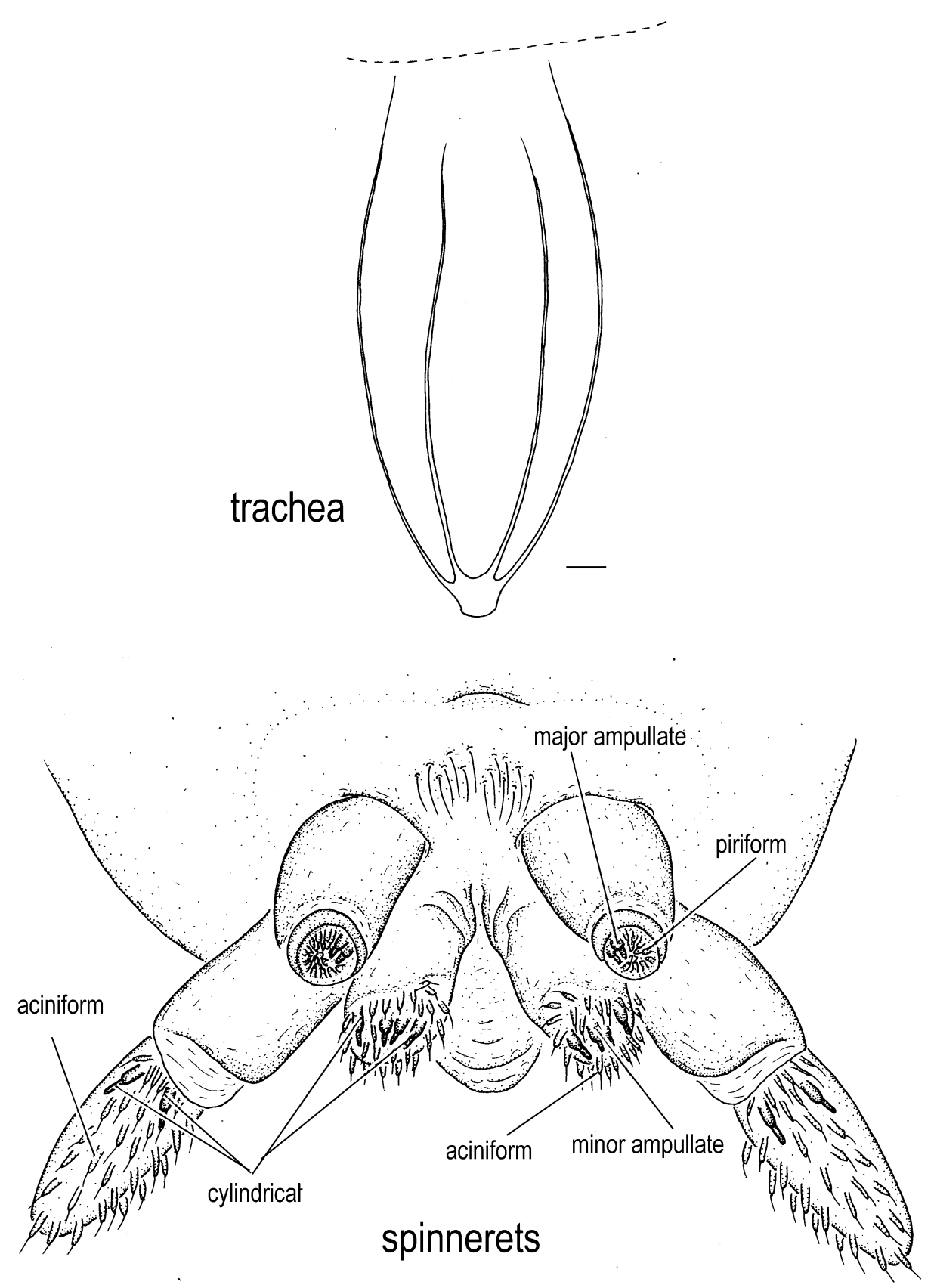

Fig. 31. Coelotes atropos (Walckenaer). Trachea and spinnerets.

Paracoelotes, fig. 324) or small (e.g., Tegecoelotes, fig. 376). The copulatory ducts may originate anteriorly, as in most coelotines, or posteriorly as in Draconarius (figs. 182, 193).

Spermathecae: These are the structures be- tween copulatory ducts and fertilization ducts. The spermathecae can be either strongly elongated as in Asiacoelotes (fig. 71), strongly elongated and highly convoluted as in many Coras species (figs. 138, 140), or short and simple as in Coelotes (fig. 39). 

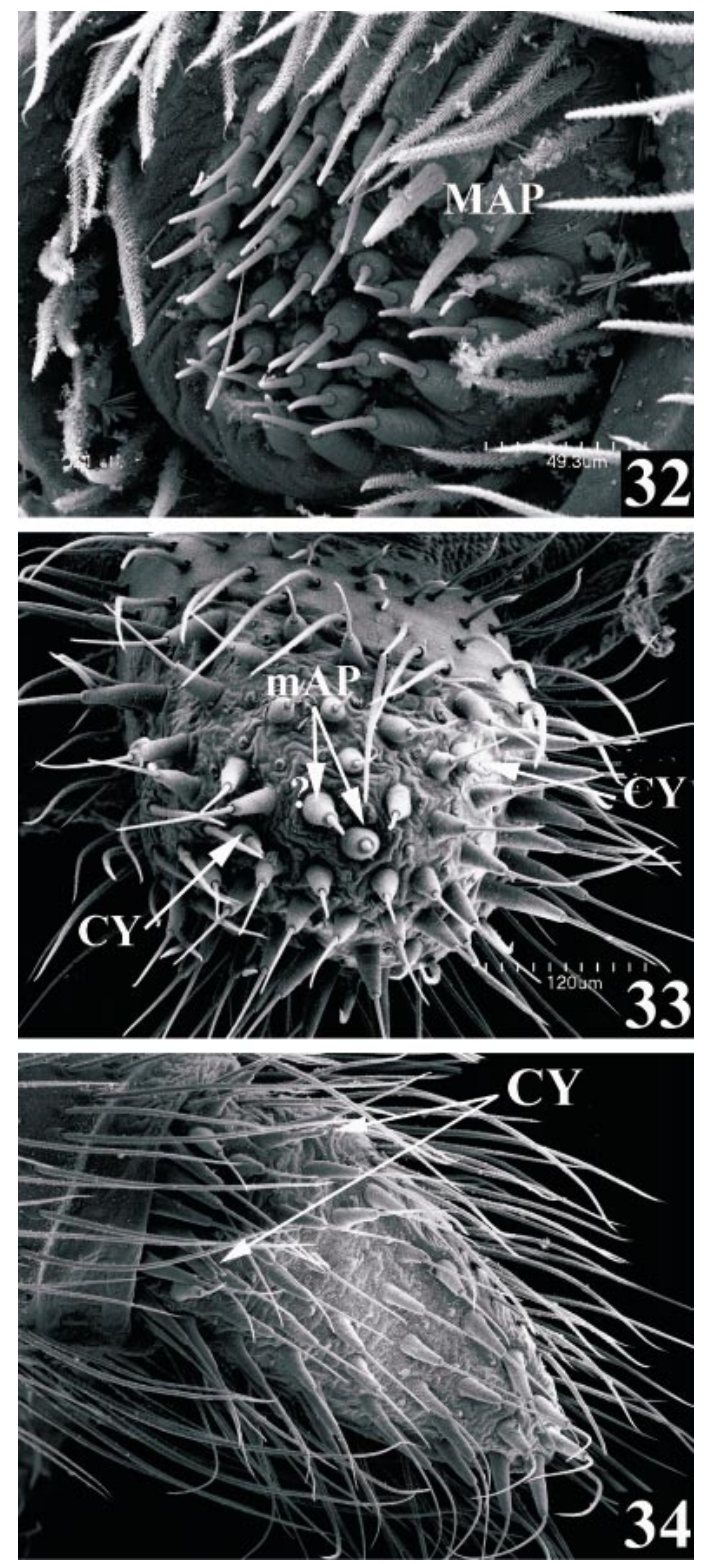

Figs. 32-34. Coelotes atropos (Walckenaer), female spinnerets. 32. ALS, left. 33. PMS, right. 34. PLS, right. Abbreviations are spelled out on p. 6.

Many coelotines possess more or less complex spermathecae of three distinct parts: spermathecal heads, stalks, and bases. Spermathecal heads are the apical, dilated parts of the spermathecae that connect to the spermathecal bases through the spermathecal


Figs. 35-37. Coelotes atropos (Walckenaer), male spinnerets, ventral view, left. 35. ALS. 36. PMS. 37. PLS.

stalks; they arise near the copulatory ducts and are usually situated anteriorly (figs. 324, 376); the size varies between species. Spermathecal stalks may be elongated, convoluted, and distinct from their bases (e.g., Coras, fig. 138). However, in many coelotines, stalks may be short or indistinct from the ba- 

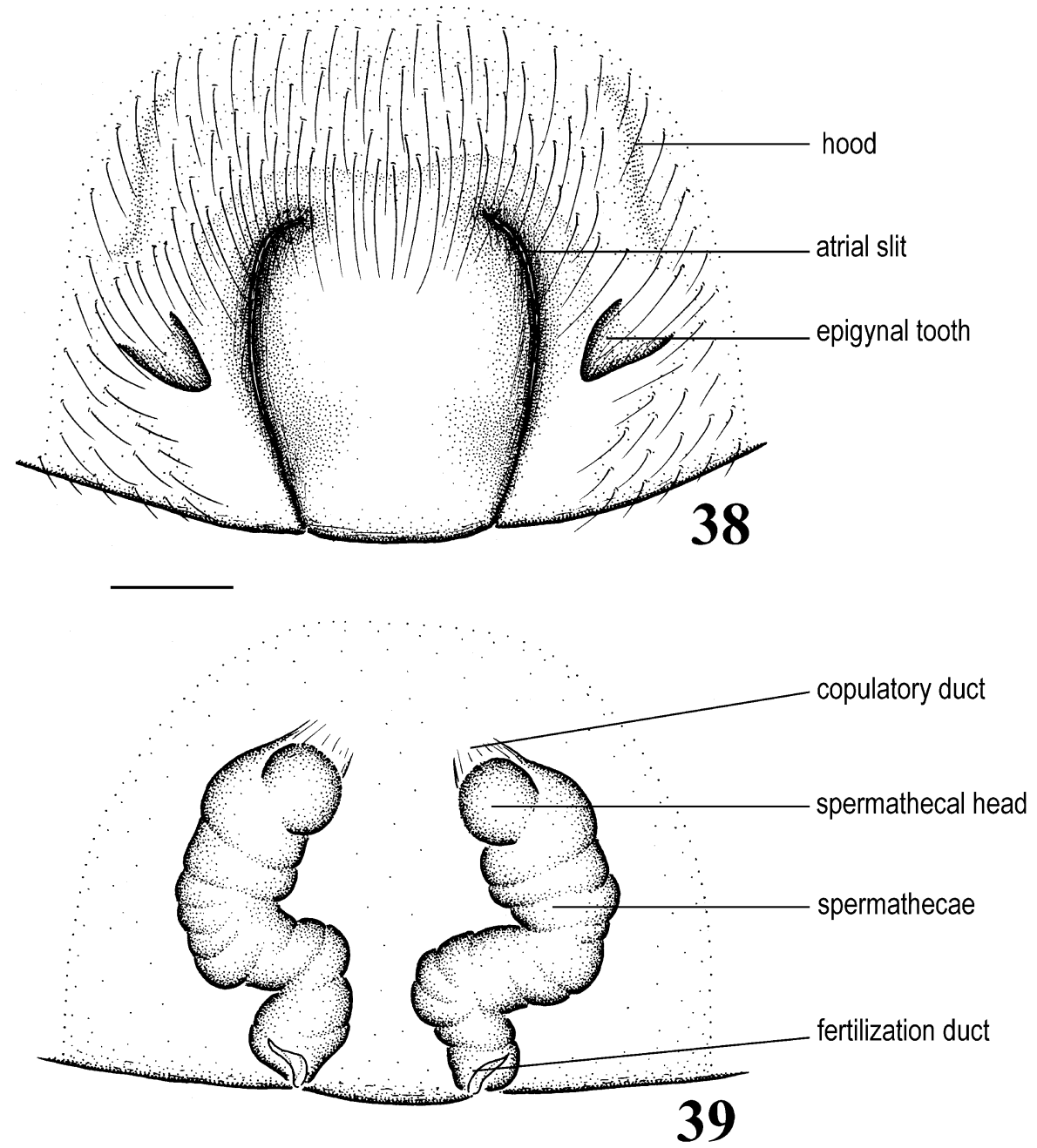

Figs. 38, 39. Coelotes atropos (Walckenaer), female. 38. Epigynum. 39. Vulva.

ses (e.g., Paracoelotes, fig. 324), which themselves are highly sclerotized, slightly convoluted, and dilated relative to the stalks.

\section{Male}

Femur: The femur has, in most coelotines, three distinctive strong spines dorsally (figs. 47, 48). In Eurocoelotes gasperinii (Simon, 1891), 5 or 6 short strong spines arise only distally and 1 arises medially. Most coelotines have no femoral apophysis, but in $\mathrm{Fe}$ moracoelotes a femoral apophysis arises distally (figs. 229, 230), and prolaterally there is cluster of numerous short, strong setae (fig. 231).
Patella: In Tegecoelotes, the patella is elongated to at least twice the tibial length (figs. 377-380). A long strong spine always arises distally on the dorsal patella, and most coelotines have a relatively short spine proximally (figs. 45, 403). A patellar apophysis may be absent (e.g., Ambanus, fig. 60) or may vary in number from one (as in most coelotines, figs. 146, 313), two (as in Coronilla, figs. $162,165,168$ ), to three (as in Robusticoelotes, figs. 353, 354). The apophysis may be small (as in Paracoelotes, fig. 326) or broad (as in Coelotes, figs. 45, 49). In the Coelotes charitonovi species group, the patellar apophysis is large, broad, and strongly 

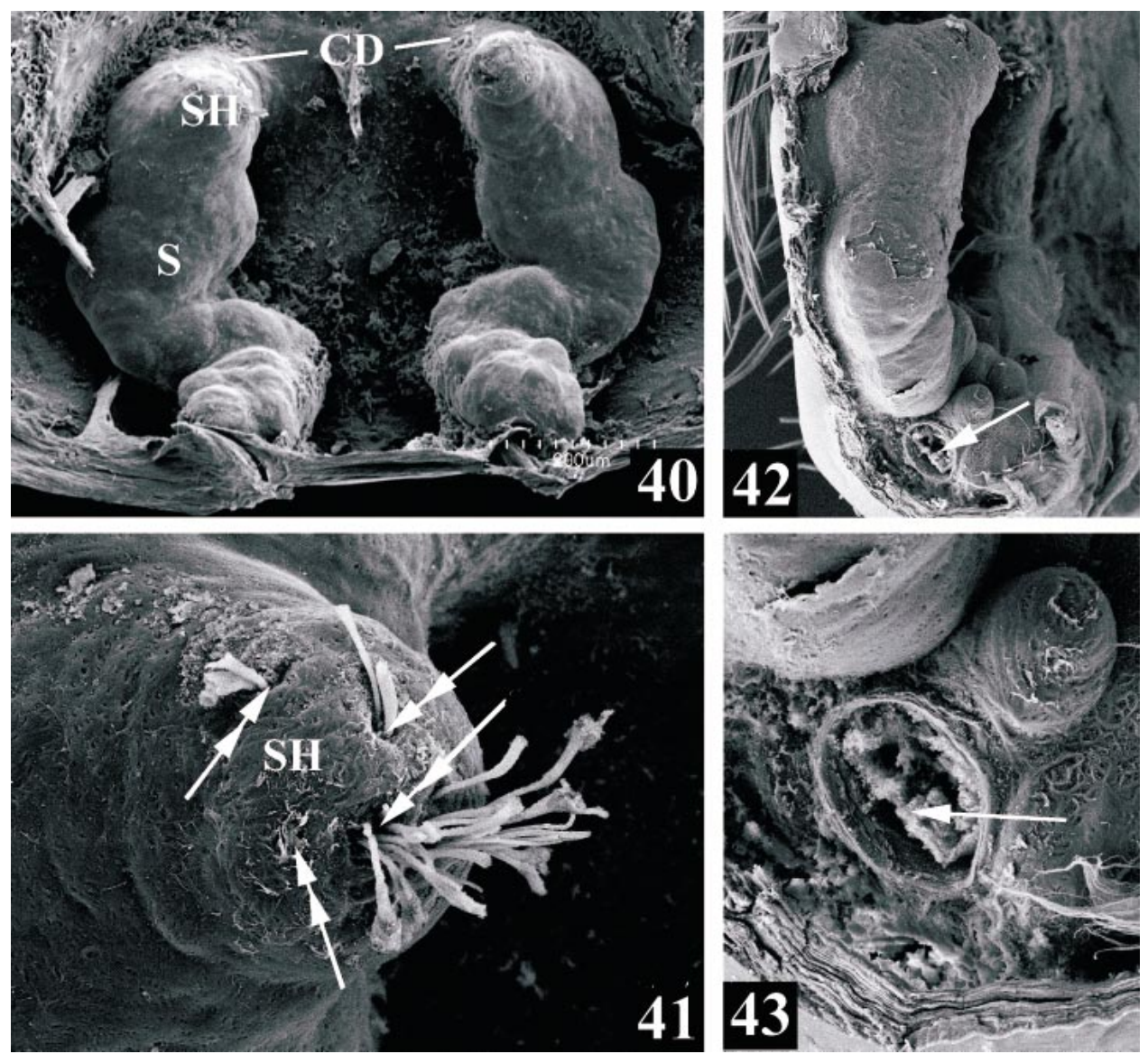

Figs. 40-43. Coelotes atropos (Walckenaer), female vulva. 40. Vulva. 41. Spermathecal head, double arrows show the pores. 42. Spermathecae, lateral view, showing the large pore at lateral side of spermathecal base. 43. Large pore at spermathecal base, enlarged. Abbreviations are spelled out on p. 6.

modified (fig. 118). In Spiricoelotes, the distal part of the apophysis is sharply bent dorsally (fig. 368). In the genus Longicoelotes, the apophysis is strongly elongated, longer than the tibia (fig. 313).

Tibia: The tibia is short, almost the same length or slightly longer than the patella. The retrolateral tibial apophysis (RTA) usually occupies most of the tibia. In Paracoelotes tianchiensis (Wang et al., 1990), the tibia is very long and the RTA occupies only onefourth to one-fifth of the tibial length. The tip of the RTA varies between species: it can be blunt, only slightly extended (fig. 368), or strongly extended anteriorly (fig. 378). Lateral tibial apophyses are present in most coelotines, with the size ranging from large (e.g.,
Tegecoelotes, fig. 378) to small (e.g., Leptocoelotes, fig. 304) or absent (e.g., Coronilla and Femoracoelotes, figs. 162, 168, 230). In Ambanus (fig. 60) and Wadotes (fig. 413), the lateral tibial apophysis is located distally on the tibia and is strongly modified into a small concavity. In Robusticoelotes (fig. 354) and some species of Coras (figs. 144, 146), the retrolateral tibial surface is also strongly modified. Three strong spines always occur on the prolateral tibial surface (figs. 46, 49, 412).

Cymbium: The retrolateral side of the cymbium is modified into a cymbial furrow in all coelotines. Similar furrows are also present in other spider families, such as Campostichomma Karsch, 1891 (Miturgidae, 

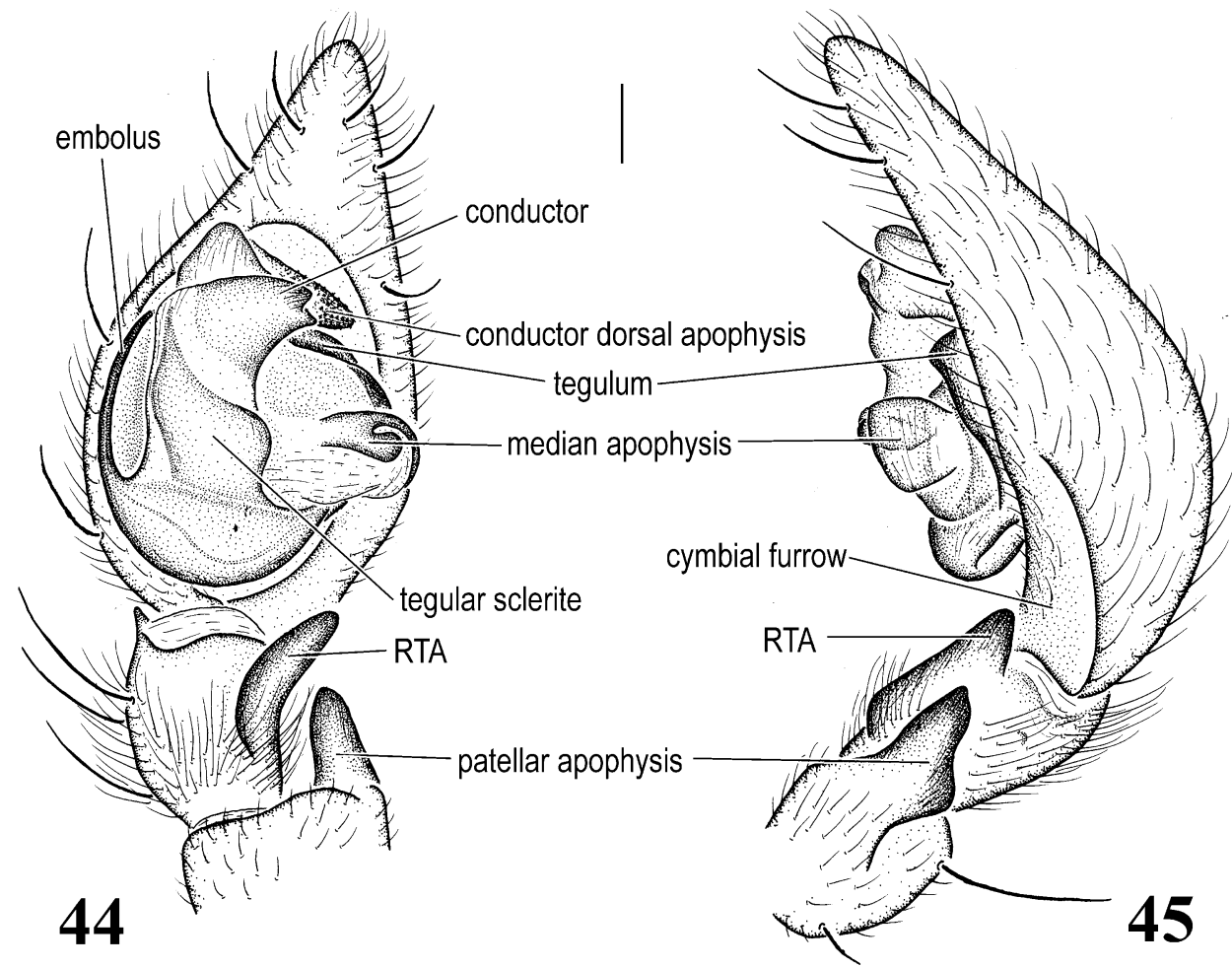

Figs. 44, 45. Coelotes atropos (Walckenaer), male palp. 44. Ventral view. 45. Retrolateral view.

see Griswold, 1993) and Mallinella Strand, 1906 (Zodariidae, see Wang et al., 1999), but with quite different palpal structures and apparently not closely related. The coelotine cymbial furrow may be short and shallow (e.g., Ambanus, Coronilla, and Longicoelotes, figs. 60, 162, 314) or strongly elongated to more than half the cymbial length, with a strongly concave dorsal edge (e.g., Asiacoelotes and Bifidocoelotes, figs. 73, 89). In all coelotines, three to six strong spines occur on the ventral distal cymbium, and four to six strong spines occur on the prolateral cymbium (figs. 44, 49), although they are not always so strong as the distal spines. In $\mathrm{Wa}$ dotes, the basal cymbium bears retrolateral and prolateral apophyses (figs. 412, 414, 415). Trichobothria are usually absent on the cymbium, but many Himalayan species of both the genus Himalcoelotes and Draconarius have a row of two to five trichobothria on the retrolateral side, just anterior to the cymbial furrow.

Basal hematodocha, subtegulum and me- dian hematodocha: All coelotines possess a large basal hematodocha with a well-developed petiolus on the retrolateral side (figs. 49, 167). The subtegulum is sclerotized, funnel-shaped, and contains the fundus of the sperm duct and three to five anelli prolaterally (figs. 49, 166); distally it is highly sclerotized. A short and relatively indistinct median hematodocha connects the distal subtegulum to the tegulum (fig. 49).

Tegulum: The tegulum and its structures differ considerably among species and genera. The ringlike wall of tegulum is highly sclerotized. The distal inflatable surface of the tegulum is relatively membranous and houses some distinct structures, namely the tegular sclerite, conductor, embolus, and median apophysis.

Tegular sclerite: In most coelotines, the tegular sclerite is simple, but some speciesspecific structures do occur. In Asiacoelotes xinhuiensis, a small sharp apophysis arises from its retrolateral side (fig. 72). In some Coras species, the anterior tegular sclerite is 


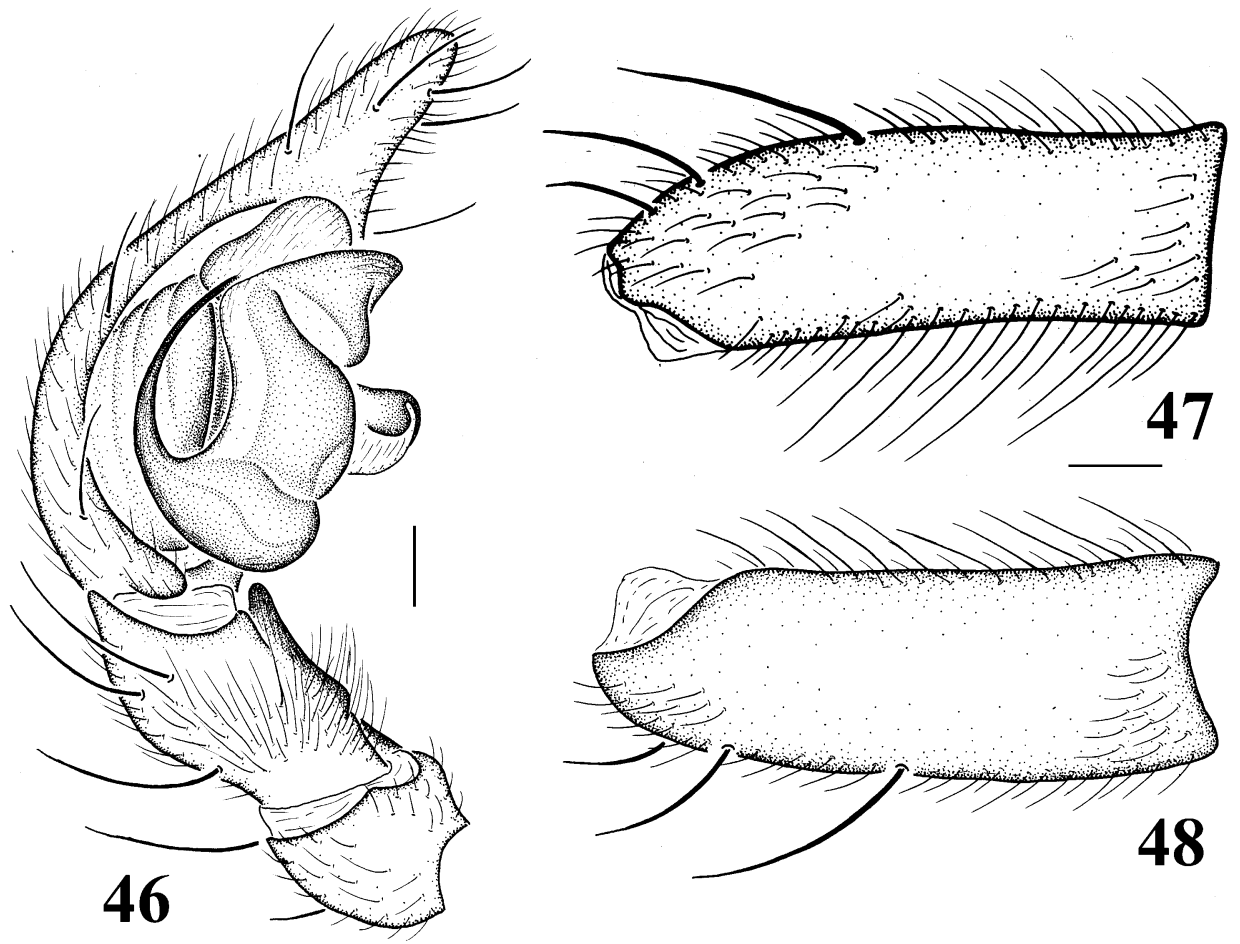

Figs. 46-48. Coelotes atropos (Walckenaer), male palp. 46. Prolateral view. 47. Femur, retrolateral view. 48. Femur, prolateral view.

prolaterally expanded and forms a broad apophysis (fig. 143). In Ambanus, the tegular sclerite is weakly sclerotized (fig. 59).

Conductor: This arises prolaterally on the anterior tegular sclerotized ring and is closely associated with the anterior part of the tegular sclerite. The conductor base is usually modified to form a membranous lamella and varies from small and moderately developed (e.g., Coelotes and Paracoelotes, figs. 44, 46,

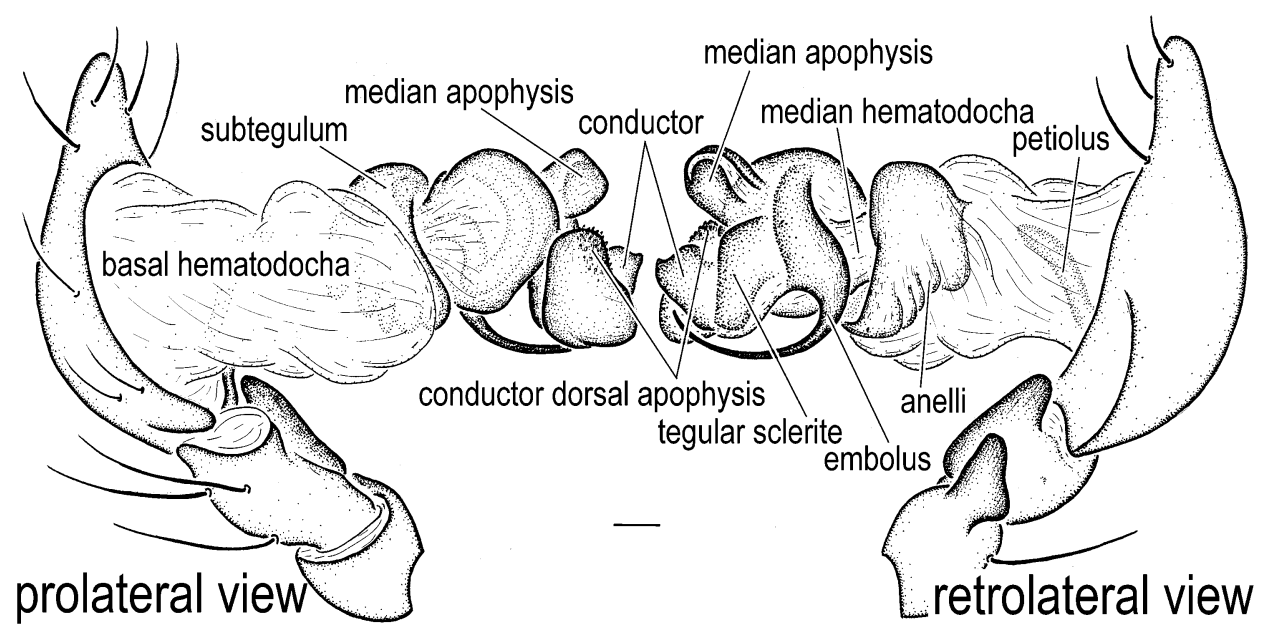

Fig. 49. Coelotes atropos (Walckenaer), male palp, expanded. 

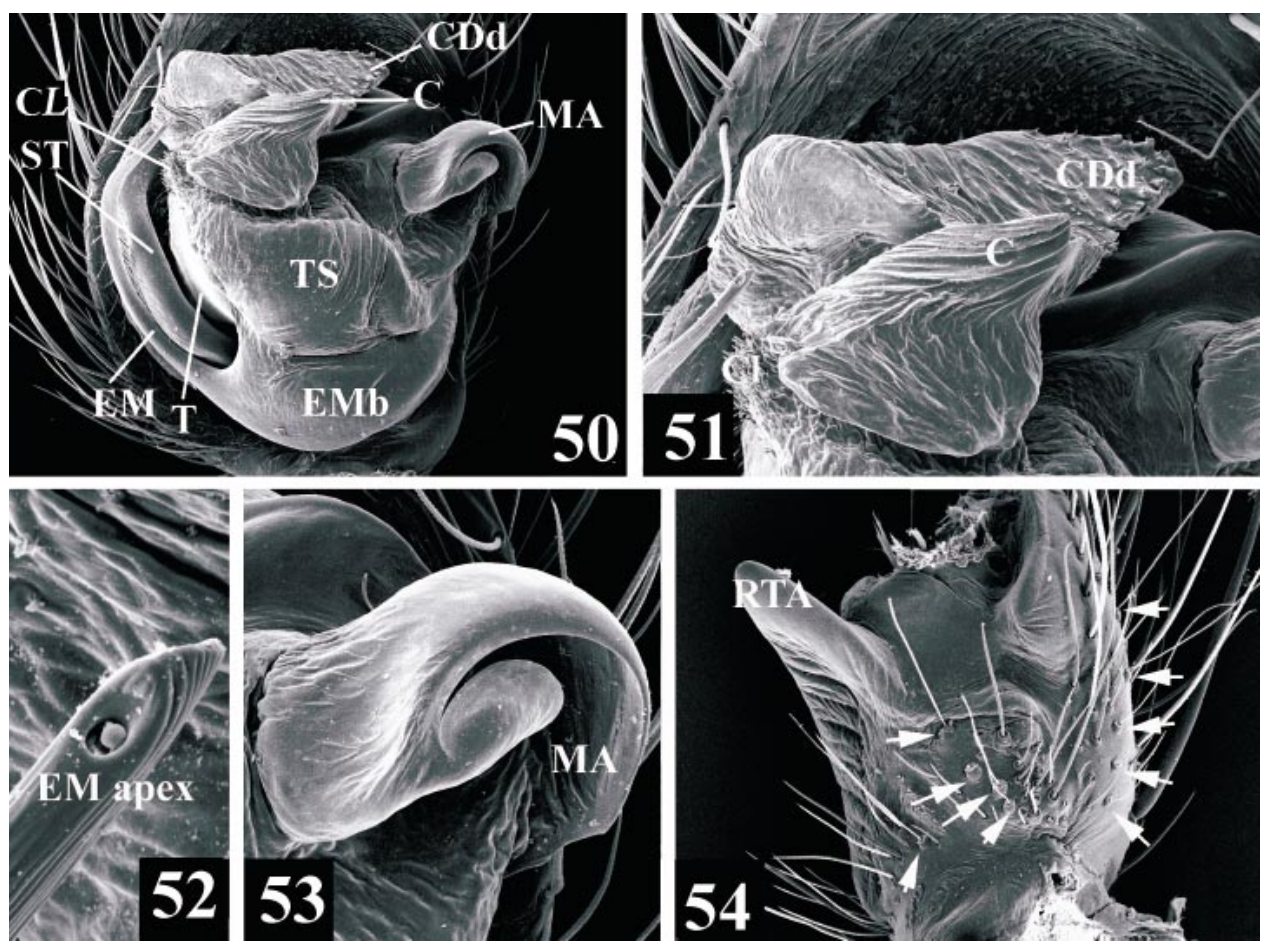

Figs. 50-54. Coelotes atropos (Walckenaer), male palp. 50. Bulb, ventral view. 51. Enlarged, showing conductor lamella (CL), conductor (C), and conductor dorsal apophysis (CDd). 52. Enlarged, showing embolus (EM) apex. 53. Enlarged, showing median apophysis (MA). 54. Tibia, showing retrolateral tibial apophysis (RTA) and tibial trichobothria (indicated by arrows). Other abbreviations: EMb, embolic base; ST, subtegulum; T, tegulum; TS, tegular sclerite. Abbreviations are spelled out on p. 6.

$325)$, to large and well developed (e.g., Asiacoelotes and Draconarius, figs. 72, 74, 183, 184). The conductor dorsal apophysis may be absent (e.g., Asiacoelotes and Paracoelotes, figs. 73,326 ) or present (as in most coelotines, figs. 44, 213). In some Coras species, an apophysis, here called the paraconductor, arises between the conductor and the conductor dorsal apophysis (figs. 143, 144). The paraconductor is similar and parallel to the conductor. In Coelotes atropos, the conductor is very short, whereas the conductor dorsal apophysis is broad and strong (fig. 44). In Draconarius sinualis, both the conductor and its dorsal apophysis are slender and long (fig. 198). The conductor of Paracoelotes is extremely long, flat, spiral, and forms one or two loops, but it lacks a conductor dorsal apophysis (figs. 325, 326). In the genus Wadotes, the conductor dorsal apophysis is strongly modified into a long, toothed process (figs. 413, 414, 416). The conductor is the male palpal structure that varies both in size and shape between taxa.

Embolus: This arises from the posterior tegular ring and is closely associated with the posterior tegular sclerite. The ejaculatory duct of the sperm duct lies inside the embolus. In coelotines, the embolus is usually linear and slender, but it can also be broad and strong (e.g., Ambanus and Robusticoelotes, figs. 59, 351). In most coelotines, the embolus is moderately long (e.g., Coelotes, fig. 44), but it can be very long (e.g., Asiacoelotes, fig. 72). The apex of the embolus usually lies inside the longitudinal groove of the conductor. The embolus may be prolateral (e.g., Coelotes and Eurocoelotes, figs. 44, 213) or posterior in origin (e.g., Asiacoelotes and Spiricoelotes, figs. 72, 367).

Median apophysis: The median apophysis in coelotines is a sclerotized structure located in the retrolateral membranous tegular surface (Bennett, 1987). In most coelotines, the 
median apophysis is spoonlike, but it can be either a very small apophysis (e.g., most Longicoelotes, fig. 313) or large but not spoonlike (as in Coronilla, fig. 162). In some species, the median apophysis is absent (e.g., Femoracoelotes). The spoonlike median apophysis may be rounded with a smooth distal end as in Coelotes (fig. 44) or with a more or less extended distal end as in Paracoelotes (figs. 325, 326). In Himalcoelotes, the median apophysis is small, rounded, medially notched, and strongly extended (figs. 267, 268). In some species, a small tegular carina may be present between the median apophysis and tegular sclerite. In Coras, the tegular carina is well developed and extends either transversely or longitudinally (figs. 143, 144).

\section{RELATIONSHIPS}

Lehtinen (1967) "defined" the subfamily Coelotinae as a monophyletic group characterized by the "strange modification of the cymbium" and further suggested that the coelotine species strongly resemble those of the cribellate amaurobiid genera Tamgrinia, Taira, and Amaurobius, both in general appearance and habits. However, few taxonomic studies have been done on coelotine genera, and none has discussed relationships among the coelotine genera.

This study is the first generic revision and phylogenetic analysis for the Coelotinae, based on 31 characters and 22 taxa (see fig. 55 ), including 2 outgroup taxa.

\section{TAXA}

For the ingroup, all 20 coelotine genera were included in the analysis. Two putatively closely related amaurobiid genera, Tamgrinia and Amaurobius, were chosen as outgroup taxa to root the tree.

\section{CHARACTERS}

For the character analysis, taxa from the Amaurobiidae, Agelenidae, Dictynidae, Hahniidae, Phyxelididae, and Titanoecidae were examined. This permitted a better understanding of coelotine characters and helped determine which are unique to the subfamily Coelotinae. After extensive search for char- acters that might resolve relationships of the coelotine genera, 31 characters were used.

Character coding for each terminal is mainly based on an exemplar species, which in most cases is the type species of the genus. Exceptions are the amaurobiid genus Tamgrinia (type species with only the male described) and the coelotine genus Draconarius (type species not available). As a result, $T$. laticeps (Schenkel, 1963) and D. wudangensis (Chen and Zhao, 1997) were chosen as exemplars. All multistate characters were treated as unordered. Missing or inapplicable data were coded as -. A list of characters follows:

Character 0. Cheliceral promarginal teeth: (0) three; (1) four or more. State 1 is present in Robusticoelotes, Spiricoelotes, Leptocoelotes, and Amaurobius.

Character 1. Cheliceral retromargin teeth: (0) two; (1) three; (2) four; (3) five or more. State 1 is present in most coelotine species, including Tegecoelotes, Coelotes, Eurocoelotes, Urocoras, Coras, and Paracoelotes. State 2 is known only in Coronilla, Femoracoelotes, and Aisacoelotes. Robusticoelotes, Leptocoelotes, and Spiricoelotes have more than four retromarginal teeth. All other coelotines known have two retromarginal teeth.

Character 2. AMS: (0) cribellum; (1) hairs. All known coelotines are ecribellate spiders.

Character 3. Calamistrum: (0) present; (1) absent. Coelotines lack a calamistrum.

Character 4. PLS with second segment: (0) short; (1) long. Ecribellate coelotines have the second segment of PLS at least as long as the first segment or longer.

Character 5. PMS cylindrical spigots: (0) two; (1) three or four. Paracoelotes has at least three cylindrical gland spigots on PMS, and Tamgrinia has four.

Character 6. Epigynal atrium: (0) large (fig. 57); (1) small. The large atrium occurs in Coronilla, Ambanus, and Robusticoelotes. Femoracoelotes, Tonsilla, Paracoelotes, and Draconarius also have a large atrium, although it is not as big as in the former three genera. All other known coelotines have the atrium reduced to atrial slits.

Character 7. Posterior extension of anterior atrial margin: (0) absent; (1) present (fig. 
323). State 1 occures only in Tonsilla and Paracoelotes.

Character 8. Atrial septum: (0) absent; (1) present. State 1 is present only in Tamgrinia and Coronilla (figs. 158, 159).

Character 9. Epigynal teeth: (0) absent; (1) present. State 1 is present in most coelotines (fig. 38), but is absent in Coronilla, Femoracoelotes, Ambanus, Robusticoelotes, Longicoelotes, Platocoelotes, and Spiricoelotes.

Character 10. Epigynal teeth: (0) one; (1) two. Only Bifidocoelotes and Wadotes have one epigynal tooth.

Character 11. Epigynal teeth: (0) short; (1) strongly elongated. State 1 is found only in Himalcoelotes and Urocoras (fig. 265).

Character 12. Epigynal teeth: (0) slender; (1) broad. Only Tegecoelotes and Leptocoelotes have broad epigynal teeth (figs. 296, 375).

Character 13. Copulatory ducts: (0) small; (1) large. Copulatory ducts small in Longicoelotes, Leptocoelotes, Tegecoelotes, Coelotes, Himalcoelotes, Coras, Asiacoelotes, Platocoelotes, and Spiricoelotes (fig. 39). Other known coelotines have large copulatory ducts (fig. 324).

Character 14. Spermathecae: (0) short (fig. 39); (1) strongly elongated, highly convoluted together; (2) strongly elongated, loosely convoluted. State 1 found only in Coras (fig. 138). State 2 found only in Draconarius, Asiacoelotes, Bifidocoelotes, Spiricoelotes, and Platocoelotes (fig. 71).

Character 15. Patellar apophysis: (0) absent; (1) present. In these data, state 1 is found in all Coelotinae (fig. 45) except Ambanus and Eurocoelotes.

Character 16. Lateral tibial apophysis: (0) absent; (1) present. In these data, state 1 is found in all coelotines (fig. 304), except $\mathrm{Co}$ ronilla and Robusticoelotes.

Character 17. Anterior part of lateral tibia: (0) normal; (1) modified with small concavity. State 1 is present in Ambanus and $\mathrm{Wa}$ dotes (figs. 60, 413).

Character 18. Tibia lateral surface modification: (0) absent; (1) present. State 1 found only in some Coras species and Robusticoelotes (figs. 146, 354).

Character 19. Cymbial furrow: (0) absent; (1) present. State 1 found in all known Coelotinae (fig. 45).
Character 20. Cymbial furrow: (0) short, less than half cymbial length (fig. 45); (1) long, more than half cymbial length in Draconarius, Asiacoelotes, Bifidocoelotes, Spiricoelotes, and Platocoelotes (fig. 89).

Character 21. Tegular sclerite: (0) absent; (1) longitudinally situated on tegulum. State 1 found in all known Coelotinae (fig. 44).

Character 22. Conductor lamella: (0) absent; (1) present. State found in all known Coelotinae (figs. 72, 108).

Character 23. Conductor lamella: (0) small (fig. 108); (1) large. State 1 found only in Wadotes, Draconarius, Asiacoelotes, Bifidocoelotes, Spiricoelotes, and Platocoelotes (fig. 72).

Character 24. Conductor dorsal apophysis: (0) absent in Leptocoelotes, Paracoelotes, Urocoras, Asiacoelotes, and Spiricoelotes; (1) present in most coelotines (figs. 44, 213, 214).

Character 25. Embolic base: (0) prolateral or anterior in origin (fig. 44); (1) posterior in origin. State 1 found only in Draconarius, Asiacoelotes, Bifidocoelotes, Spiricoelotes, and Platocoelotes (fig. 72).

Character 26. Embolus: (0) short (fig. 44); (1) extremely long. State 1 found only in Draconarius, Asiacoelotes, Bifidocoelotes, Spiricoelotes, and Platocoelotes (fig. 72).

Character 27. Embolus: (0) linear; (1) broad, as in Femoracoelotes, Ambanus, Robusticoelotes, and Urocoras (fig. 59).

Character 28. Median apophysis: (0) absent; (1) present. Most coelotines have a median apophysis (figs. 44, 162, 386), but it is absent in Femoracoelotes, Leptocoelotes, Platocoelotes, and Spiricoelotes (figs. 303, 304).

Character 29. Median apophysis: (0) not spoonlike (fig. 162); (1) spoonlike, as in most coelotines, including Coelotes, Eurocoelotes, Coras, Himalcoelotes, Urocoras, Tonsilla, Paracoelotes, Wadotes, Draconarius, Asiacoelotes, and Bifidocoelotes (fig. 44).

Character 30. Spoonlike median apophysis: (0) with smooth, rounded distal end (fig. 44); (1) with strongly extended distal end, as in Coras, Tonsilla, Paracoelotes, Wadotes, Draconarius, and Asiacoelotes (fig. 386). 


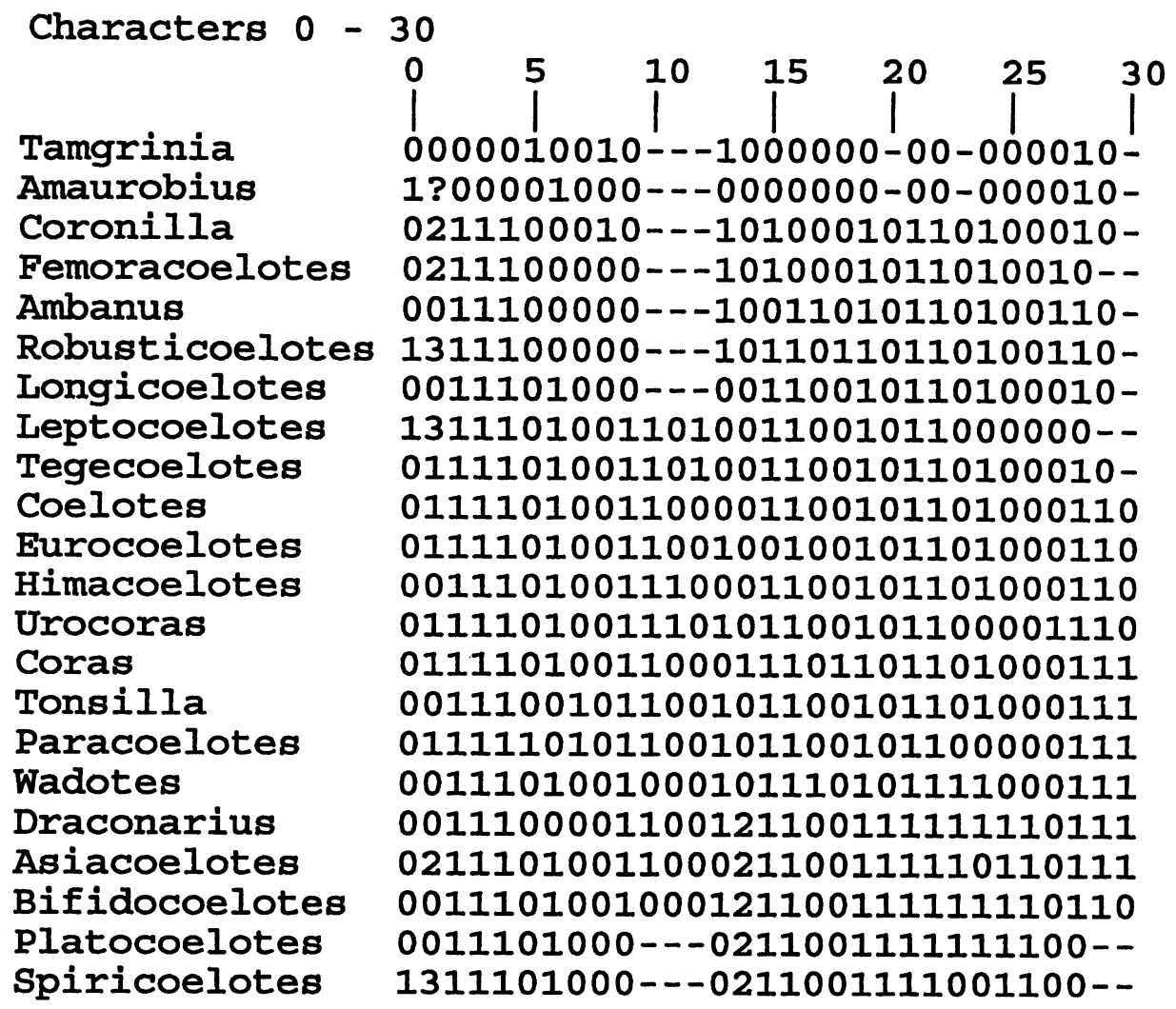

Fig. 55. Character matrix for 22 genera. See text for character description.

\section{ANALYSIS}

The data (fig. 55) were first run using Hennig86, version 1.5 (Farris, 1988) using the $\mathrm{mh}^{*}$ and $\mathrm{bb}^{*}$ tree-generating algorithms and branch swapping, which produced a total of 2102 cladograms (length 70, CI 0.48, RI $0.63)$. This suggests that some characters are homoplasious, and as a result do not deserve as much weight as others. Two rounds of successive approximations weighting (Farris, 1969) produced a stable solution of 60 cladograms, with length 202, CI 0.87 , and RI 0.94. Among these resulting cladograms, 19 have the genus Tegecoelotes sister to node 5 (of the preferred tree, fig. 56), but they were supported only by a highly homoplasious character (presence of three retromarginal cheliceral teeth). Of the remaining 41 trees, 20 of them support a sister-group relationship between the genera Leptocoelotes and Tegecoelotes based on broad epigynal teeth. The broad epigynal teeth of Tegecoelotes are strong, highly sclerotized, whereas the genus Leptocoelotes has weak, slightly sclerotized epigynal teeth. The differences between these two genera in epigynal teeth and male palp suggest that they may not form a monophyletic group.

In 8 of the remaining 19 trees, a highly homoplasious character (presence of three retromarginal cheliceral teeth) weakly supports either the relationship among Coras, Coelotes, Eurocoelotes, node 12, or the relationship among Coelotes, Eurocoelotes, node 12. In 9 other trees, the genus Coras and node 11 (genera Tonsilla and Paracoelotes) remain unresolved either with Coelotes, Eucoelotes, node 12 , or with node 8 .

The differences between the remaining four cladograms are only in the relationship of node 9 taxa. Two show Bifidocoelotes as sister to Platocoelotes + Spiricoelotes, but they were weakly supported by the smooth distal end of the median apophysis (median 




Fig. 56. Preferred cladogram for 22 genera. See text for discussion.

apophysis absent in both Platocoelotes and Spiricoelotes). Two remaining cladograms, after collapsing an unsupported clade, are the same topology, which was chosen as the preferred tree for final analysis (fig. 56).

Implied weighting (Goloboff, 1993a) using PeeWee, version 2.6 (Goloboff, 1997), with the concavity function settings $1-5$ of the homoplasy, found the same eight trees, and one of them is same as figure 56 except that Asiacoelotes is weakly supported as sister group to Platocoelotes + Spiricoelotes by the presence of small copulatory ducts. The concavity function setting 6 also found 8 trees but three of them are different from the setting 1-5 trees. One of the "setting 6 trees" is same as figure 56 except that Tegecoelotes is supported as sister group to Leptocoelotes, and Asiacoelotes is weakly supported as sister group to Platocoelotes + Spiricoelotes.

Successive approximations weighting on the trees produced by Nona, version 1.8 (Goloboff, 1993b) resulted in 8 trees and stabilized in the second iteration. One of them is same as figure 56 except that Tegecoelotes is weakly supported as sister group to node 5 by the presence of three retromarginal cheliceral teeth, and Asiacoelotes is weakly supported as sister group to Platocoelotes + Spiricoelotes by the presence of small copulatory ducts. The remaining cladograms show no improvements when compared to the preferred tree (fig. 56).

\section{CONCLUSIONS}

The subfamily Coelotinae is resolved at node 1 , supported by loss of the cribellum and calmistrum and by the presence of the elongated PLS second segment, the patellar apophysis (secondarily lost in Ambanus and Eurocoelotes), the cymbial furrow, the lon- 
gitudinally extended tegular sclerite on the tegulum, the conductor lamella at the conductor base, and the conductor dorsal apophysis (lost in Leptocoelotes, Paracoelotes, Urocoras, Eurocoelotes, and Asiacoelotes).

Within the Coelotinae, Coronilla and $\mathrm{Fe}$ moracoelotes are united at node 14 as the sister group of all other coelotines; this is supported by the presence of four cheliceral retromargin teeth (paralleled in Asiacoelotes).

The presence of a small apophysis on the lateral side of the tibia (lateral tibial apophysis) unites the remaining coelotines at node 2.

Ambanus + Robusticoelotes (node 13) is supported by the broad embolus (parallel in Femoracoelotes and Urocoras), and is the sister group to other node 2 coelotines.

Two characters, reduced atrium (reversed to large atrium in Draconarius, Tonsilla, and Paracoelotes) and small copulatory ducts (reversed to large copulatory ducts in Eurocoelotes, Urocoras, Wadotes, Draconarius, Bifidocoelotes, Tonsilla, and Paracoelotes), support a group (at node 3) including Longicoelotes plus all coelotines with epigynal teeth at node 4.

Node 4 is supported by presence of epigynal teeth on the female epigynum (lost in Platocoelotes and Spiricoelotes) and by the presence of three cheliceral retromarginal teeth (highly homoplasious, reversed to two, four, or five teeth in nine genera).

Node 4 comprises most coelotine species, with Leptocoelotes and Tegecoelotes remaining unresolved. Although both Leptocoelotes and Tegecoelotes have broad epigynal teeth, they may not be homologous. In fact, the broad epigynal teeth of Leptocoelotes are very weak, less sclerotized, whereas those of Tegecoelotes are robust and highly sclerotized.

The remaining node 4 coelotines cluster together at node 5 by presence of a spoonlike median apophysis and slender, normal epigynal teeth. Coelotes and Eurocoelotes remain unresolved here with node 12 and node 6.

The presence of strongly elongated epigynal teeth clusters Himalcoelotes and Urocoras at node 12 .

Node 6 is supported by strongly extended distal end of the median apophysis (reversed to smooth, rounded distal end in the genus Bifidocoelotes).

Node 11 and node 8 are united together at node 7 by presence of large copulatory ducts (reverse to small copulatory ducts in Asiacoelotes, Platocoelotes, and Spiricoelotes). Tonsilla and Paracoelotes are monophyletic, supported at node 11 by presence of a large atrium and posteriorly extended anterior atrial margin.

Node 8 is supported by presence of two cheliceral retromarginal teeth (reversed to four or five teeth in Asiacoelotes and Spiricoelotes) and by strongly developed, enlarged conductor lamella at the conductor base. This node supports the sister-group relationship between Wadotes and the node 9 coelotines.

Node 9 is supported by strongly elongated spermathecal tubes, long, strongly extended cymbial furrow, posteriorly originating embolic base, and long embolus. The genera Draconarius, Asiacoelotes, and Bifidocoelotes remain unresolved. The absence of both epigynal teeth and median apophysis unites Platocoelotes and Spiricoelotes at node 10.

\section{SYSTEMATICS}

\section{Coelotinae F.O.P.-CAMBridge}

Coelotinae F.O.P.-Cambridge, 1893: 158. (type genus Coelotes Blackwall). - Lehtinen, 1967: 350.

Diagnosis: Ecribellate spiders. Coelotines differ from other amaurobiids by the following combination of characters: the presence of patellar apophyses, a cymbial furrow, the tegular sclerite, a conductor lamella, the spoonlike median apophysis (in most species) of males (figs. 44-46), and the distinct epigynal teeth (in most species) of females (fig. 38). The female spinnerets have 2 cylindrical gland spigots on the PMS (except some species of Paracoelotes, with 3 or 4 cylindrical spigots) and 2 on PLS (except some species of Ambanus, with 3 cylindrical spigots). The PLS are long, with the second segment at least the length of the first segment (figs. 31, 81, 82, 373, 374).

Distribution: Coelotine spiders are common in North America west to the Rocky Mountains and north to southern Canada, in 
Europe north to southern Sweden and Finland, and in Asia north to southern far eastern Russia and south to Nepal and northern Vietnam (fig. 1).

Composition: Twenty genera, including 10 new genera.

\section{KEY TO GENERA}

\section{Males}

1. Femoral apophysis absent .........2

- Femoral apophysis present (fig. 230) ... ................Femoracoelotes

2. Median apophysis simple, not spoonlike (figs. 162, 378) or absent (fig. 304) ....... 3

- Median apophysis spoonlike (figs. 44, 213, 268, 386) ............. 10

3. Patella strongly elongated, at least twice tibial length (fig. 378) ......... Tegecoelotes

- Patella normal, about same length as tibia . ..................... 4

4. Patellar apophysis present ........ 5

- Patellar apophysis absent (fig. 60) ..... .................. Ambanus

5. Lateral tibia strongly modified; embolus broad; patella with three apophyses (figs. 351, 354) ......... Robusticoelotes

- Lateral tibia not modified; embolus linear, patella with only one or two apophyses (fig. 162) .................6 6

6. Median apophysis long, with apex slightly curved (figs. 161, 162), lateral tibial apophysis absent ............ Coronilla

- Median apophysis short, not pronounced (fig. 313 ) or absent (figs. 304, 339, 368), lateral tibial apophysis present (figs. 339, 304) . . .................... 7

7. Conductor with a long, strong, posteriorly extended conductor posterior apophysis (figs. 338,340 ), patella usually with two patellar apophyses (sometimes one of them very small) (fig. 339) ........ Platocoelotes

- Conductor without conductor posterior apophysis, patellar always with one patellar apophysis (figs. 304, 314, 368) .... . 8 8

8. Conductor complex, strongly bifurcated; patellar apophysis with apex straight (figs. $303,304,313) \ldots \ldots \ldots \ldots \ldots \ldots 9$

- Conductor simple, spiral, anteriorly extended; patellar apophysis with apex sharply curved dorsally (figs. 367, 368) ..... ................. Spiricoelotes

9. Patellar apophysis strongly elongated to at least tibial length or longer, extended anteriorly (figs. 313, 314) ... Longicoelotes

- Patellar apophysis short, extended dorsally (fig. 304) ........... Leptocoelotes
10. Cymbium with proximal cymbial apophysis (figs. 412, 413) .......... Wadotes

- Cymbium without proximal cymbial apophysis .................. 11

11. Spoonlike median apophysis small, rounded, medially notched, and extended strongly (figs. 267, 268) ....... Himalcoelotes

- Spoonlike median apophysis normal (figs. $109,213,325) \ldots \ldots \ldots \ldots \ldots 12$

12. Cymbial furrow more than half of cymbial length; embolic base with posterior origin; embolus extremely long (figs. 72, 73, 88, $89,184,185,198,199) \ldots \ldots \ldots 13$

- Cymbial furrow short; embolic base with prolateral origin; embolus short ... 15

13. Conductor dorsal apophysis absent (figs. 72, 73) ............ Asiacoelotes

- Conductor dorsal apophysis present (figs. $89,184,185,198,199) \ldots \ldots \ldots 14$

14. Conductor simple (figs. 184, 198) ....

................ Draconarius

- Conductor broad, spiraled, with bifid apex (fig. 88) ........... Bifidocoelotes

15. Conductor strongly elongated, broad, flat, spiral with one to two apparent loops (figs. 325, 326) ....... Paracoelotes

- Conductor short .............. 16

16. Median apophysis with smooth distal end (figs. 44, 45, 213, 214, 402, 403) . . 17

- Median apophysis with distal end strongly extended, forming a sharp apophysis ...

..................... 19

17. Patellar apophysis present (figs. 45, 403) .. $\ldots \ldots \ldots \ldots \ldots \ldots \ldots \ldots$

- Patellar apophysis absent (fig. 214) ... .................Eurocoelotes

18. Patellar apophysis broad; embolus slender (figs. 44, 45, 107, 116, 130) . . Coelotes

- Patellar apophysis slender; embolus wide (figs. 403, 404) ......... Urocoras

19. Conductor with posterior lobe; patellar apophysis long, dorsally curved; conductor dorsal apophysis slender (figs. 386, 387); AME < ALE (fig. 389); cheliceral retromargin with 2 teeth (fig. 390) .... ................... Tonsilla

- Conductor without posterior lobe; patellar apophysis short, if long then not dorsally curved; conductor dorsal apophysis broad (figs. 143, 144); AME > ALE (fig. 147); cheliceral retromargin with 3 teeth (fig. 148) ................. Coras

\section{Females}

1. Epigynal teeth absent (figs. 57, 349, 360) .. 
- Epigynal teeth present (figs. 38, 265, 296,

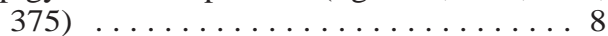

2. Atrium large (figs. 57, 158, 227, 349); copulatory ducts large (figs. 58, 160, 228, 350) $\ldots \ldots \ldots \ldots \ldots \ldots \ldots$

- Atrium small (figs. 310, 336, 360); copulatory ducts short (figs. $311,337,361$ ) . .6

3. Epigynum with posterior margin strongly expanded posteriorly; atrial septum present, transversely extended (figs. 158, 159); spermathecae close together (fig. 160) ... ................... Coronilla

- Epigynum with posterior margin not expanded; atrial septum absent; spermathecae widely separated ............ 4

4. Spermathecae slender, long, with distinct stalks (fig. 350) ...... Robusticoelotes

- Spermathecae broad, short, with stalks and bases indistinct (figs. 58, 228) . . . . 5 5

5. Spermathecae longitudinally extended (fig. 228) ............. Femoracoelotes

- Spermathecae transversely extended (fig. 58)

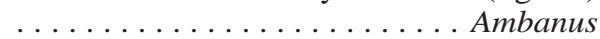

6. Epigynum strongly enlarged to occupy most epigynal plate; epigynal hoods situated posteriorly close to epigastric furrow (fig. 336) ............. Platocoelotes

- Epigynum and epigynal hoods situated anteriorly $\ldots \ldots \ldots \ldots \ldots \ldots \ldots \ldots$

7. Epigynum modified to form a round, sclerotized plate (fig. 310); spermathecae moderately long (fig. 311) .... Longicoelotes

- Epigynal normal, without median plate (fig. 360); spermathecae strongly elongated, highly convoluted (figs. 361, 363) ... ............... Spiricoelotes

8. Epigynum with single tooth (figs. 86,410 ) $\ldots \ldots \ldots \ldots \ldots \ldots$

- Epigynum with two teeth . ....... 10

9. Epigynal tooth broad, with apex not bifid or slightly bifid (fig. 410); spermathecae short, rounded (fig. 411) . . . . . . . Wadotes

- Epigynal tooth slender, with apex strongly bifid (fig. 86); spermathecae strongly elongated longitudinally (fig. 87) ........ ............... Bifidocoelotes

10. Epigynal teeth strongly elongated (figs. 265,

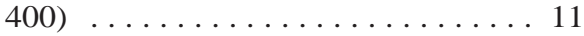

- Epigynal teeth normal length (figs. 38, 323, $296,375) \ldots \ldots \ldots \ldots \ldots \ldots \ldots 12$

11. Copulatory ducts large (fig. 401) ..... .................. Urocoras

- Copulatory ducts small (figs. 251, 255), or not visible (fig. 266) .... Himalcoelotes

12. Epigynal teeth broad (figs. 296, 375) . . 13

- Epigynal teeth small or slender (figs. 38, 211) ................. 14
13. Broad epigynal teeth strongly sclerotized, apparent (fig. 375) . . . . . . Tegecoelotes

- Broad epigynal teeth slightly sclerotized, weak (fig. 296) . . . . . . Leptocoelotes

14. Lateral atrial margins wider apart anteriorly, and convergent posteriorly (fig. 137) ...

................ Coras

- Lateral atrial margins parallel to each other, or more or less wider apart posteriorly (figs. 38, 181, 211, 323, 384), or not apparent (fig. 70) . . . . . . . . . . 15

15. Copulatory ducts large (figs.182, 212, 324, 385) ................ 16

- Copulatory ducts short (figs. 39, 71) . 19

16. Copulatory ducts with posterior origin (figs. 182, 193) ........... Draconarius

- Copulatory ducts with median or anterior origin (figs. 212, 324, 385) . . . . . . 17

17. Anterior atrial margin with posterior exten-

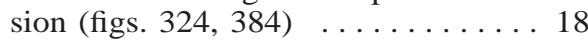

- Anterior atrial margin without posterior extension (fig. 211) ....... Eurocoelotes

18. Epigynal teeth situated close together on anterior atrial margin (fig. 384) ......

Tonsilla

- Epigynal teeth widely separated (fig. 323) ............. Paracoelotes

19. Epigynal teeth situated anteriorly (fig. 70); spermathecae strongly elongated, convoluted (fig. 71) ...........Asiacoelotes

- Epigynal teeth situated laterally (fig. 38); spermathecae short, not strongly convoluted (fig. 39) ............ Coelotes

\section{AMBANUS OVTCHINNIKOV}

Ambanus Ovtchinnikov, 1999: 63.

TyPe SPECIEs: Ambanus mandzhuricus Ovtchinnikov, 1999, by original designation.

Diagnosis: Females resemble Robusticoelotes in having a large epigynal atrium, broad copulatory ducts, and in lacking epigynal teeth, but they have short, posteriorly situated spermathecae (figs. 57, 58). Males lack a patellar apophysis and have a modified lateral tibial apophysis (figs. 59-61). Both sexes have only three promarginal and two retromarginal cheliceral teeth (fig. 63), unlike those of Robusticoelotes, which have more than five promarginal and retromarginal cheliceral teeth.

DESCRIPTION: See description of type species (below).

Distribution: Northeastern China, far eastern Russia, Japan, Korea (map 1).

Composition: Eighteen species are included, including 6 new combinations. 

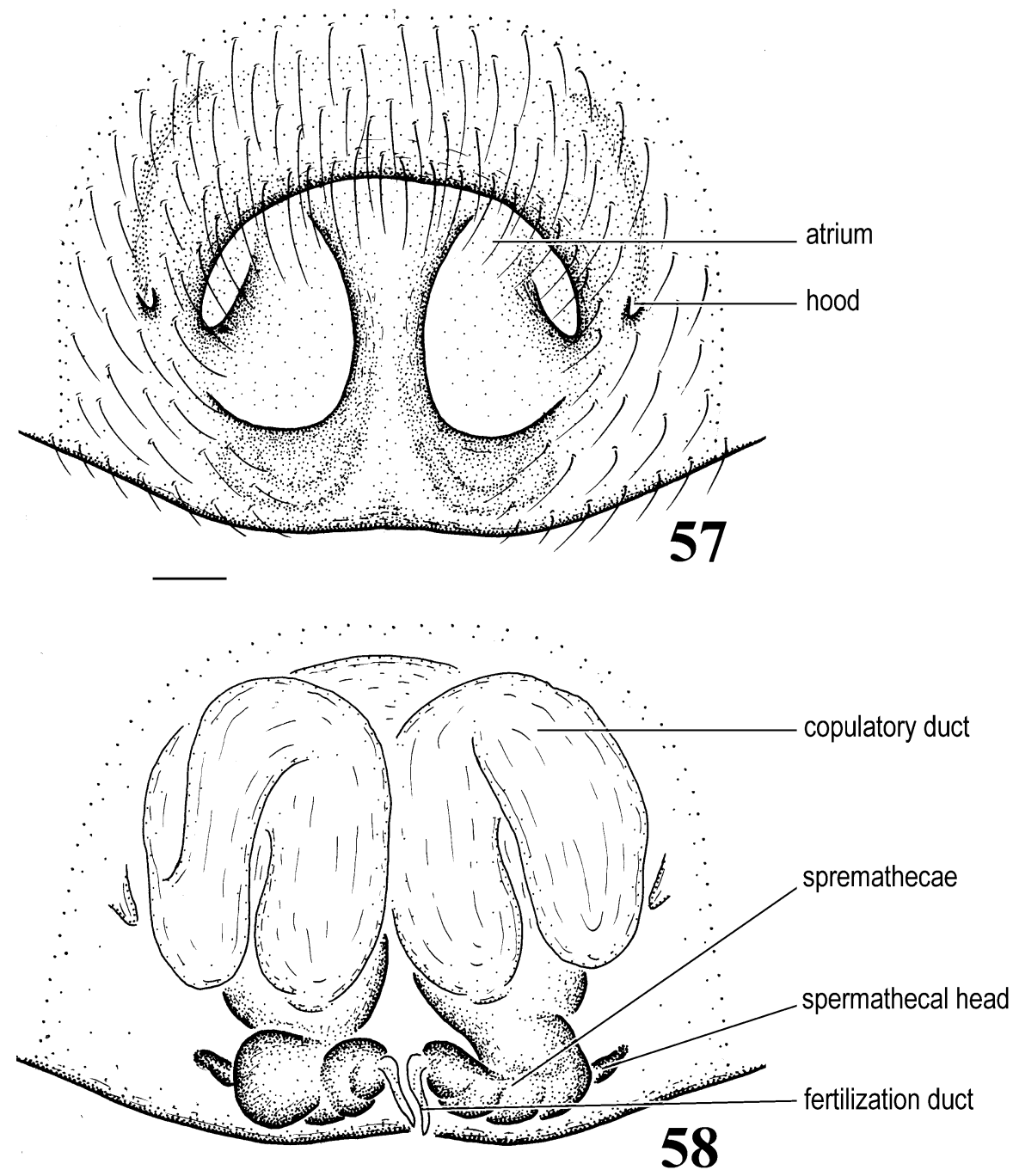

Figs. 57, 58. Ambanus mandzhuricus Ovtchinnikov, female. 57. Epigynum. 58. Vulva.

1. Ambanus amurensis Ovtchinnikov, 1999: female holotype from Khaborovsk, far eastern Russia, in CSO, not examined.

2. Ambanus bifidus (Paik, 1976): female holotype from Mt. Kaya, Korea, in Kyungpook National University, Korea, not examined. NEW COMBINATION (transferred here from Coelotes).

3. Ambanus circinalis (Gao et al., 1993): One male paratype from Liaoning, China, in IZB, examined. NEW COMBINATION (transferred here from Coelotes).

4. Ambanus dimidiatus (Paik, 1974): male holotype, male and female paratypes from
Mt. Pargong, Dongwha-sa, Korea, in Kyungpook National University, Korea, not examined.

5. Ambanus euini (Paik, 1976): female holotype and paratypes from Mt. Ilwol, Korea, in Kyungpook National University, Korea, not examined.

6. Ambanus grandivulvus (Yaginuma, 1969): female holotype from Mt. Mitake, Tsushima, Nagasaki, in NSMT, examined.

7. Ambanus kayasanensis (Paik, 1972): male holotype from Korea, in Kyungpook National University, Korea, not examined.

8. Ambanus kimi (Paik, 1974): male ho- 




Figs. 59, 60. Ambanus mandzhuricus Ovtchinnikov, male palp. 59. Ventral view. 60. Retrolateral view.

lotype and paratypes from Mt. Kaya and Mt. Pargong, Korea, in Kyungpook National University, Korea, not examined.

9. Ambanus lunatus (Paik, 1976): female holotype and paratypes from Mt. Sokkri and Kwangreung, Korea, in Kyungpook National University, Korea, not examined. One female specimen from Korea, in KAI, examined.

10. Ambanus mandzhuricus Ovtchinnikov, 1999: male and female paratypes from Kedrovaya Pad Reservation, S-Primorie, far eastern Russia, in SZM, examined.

11. Ambanus meniscatus (Zhu and Wang, 1991): female holotype and paratypes from Tonghua, Jilin, China, in NBUMS, not examined. NEW COMBINATION (transferred here from Coelotes).

12. Ambanus napolovi Ovtchinnikov, 1999: male and female paratypes from Jasnoje viu, Przhewalsky Mts. East end, S-Primorie, far eastern Russia, in AMNH, examined).

13. Ambanus ovatus (Paik, 1976): female holotype from Mt. So-Paik, Korea, in
Kyungpook National University, Korea, not examined.

14. Ambanus paiki Ovtchinnikov, 1999: male and female paratypes from far eastern Russia, in SZM, examined.

15. Ambanus paikwunensis (Kim and Jung, 1993): male holotype and female paratype from Mt. Paikwunsan Valley, Ildongmyon, Pochon-gun, Korea, in KAI, not examined. NEW COMBINATION (transferred here from Coelotes).

16. Ambanus quadrativulvus (Paik, 1974): male holotype, male and female paratypes from Paikrok-dam, Mt. Hanra, Quelpart Isl., Korea, in Kyungpook National University, Korea, not examined. Male and female specimens from Cheju-do, Korea, in KAI, examined.

17. Ambanus rostratus (Song et al., 1993): male paratype from Huanren, Liaoning, China, in IZB, examined. NEW COMBINATION (transferred here from Coelotes). One female specimen from Liaoning, China, in IZB, examined. 

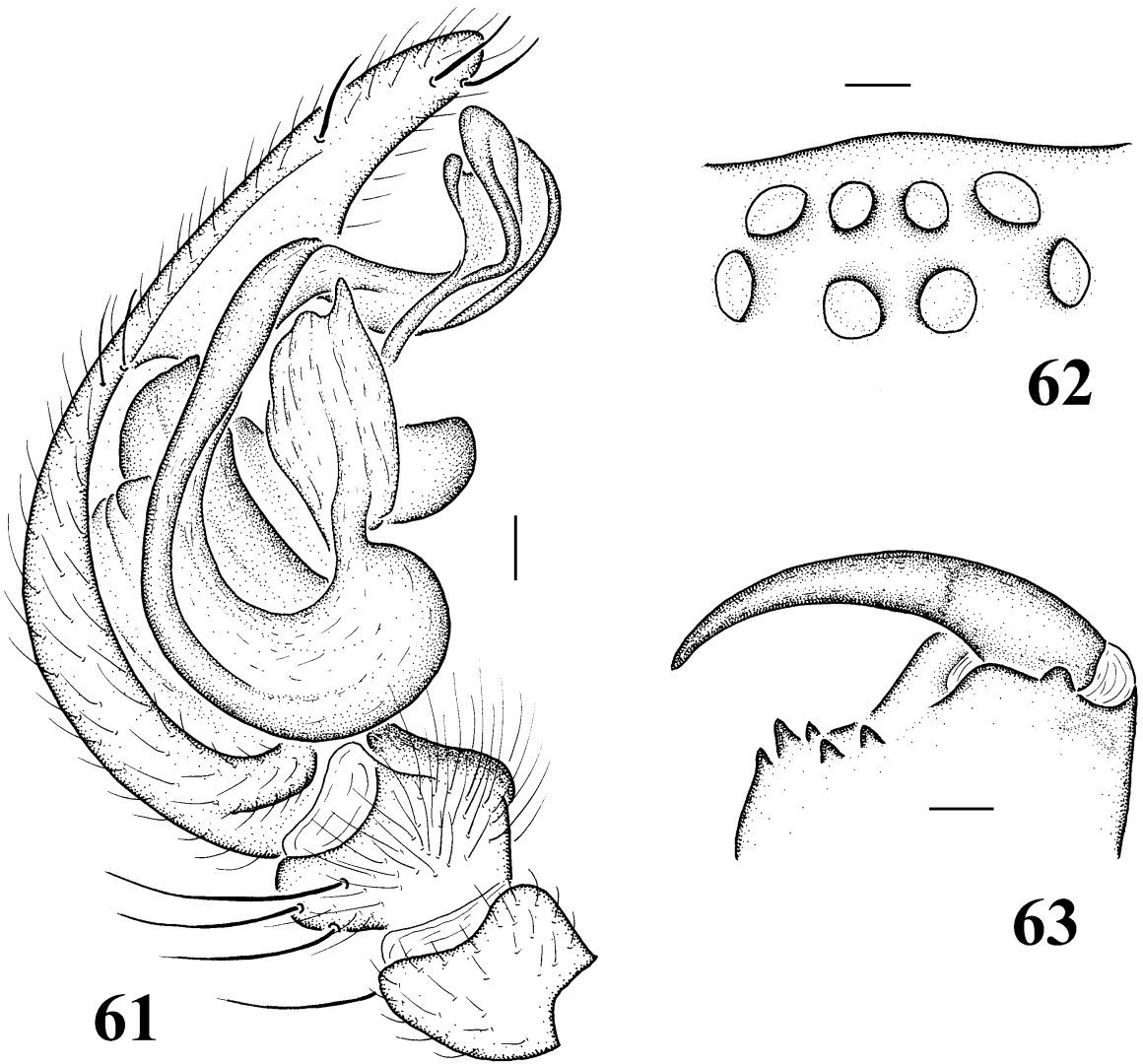

62

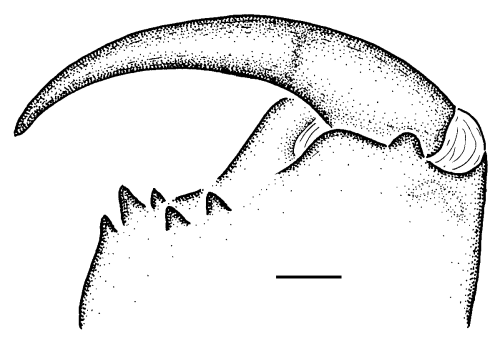

63

Figs. 61-63. Ambanus mandzhuricus Ovtchinnikov. 61. Male palp, prolateral view. 62. Eyes, dorsal view. 63. Chelicera, ventral view.

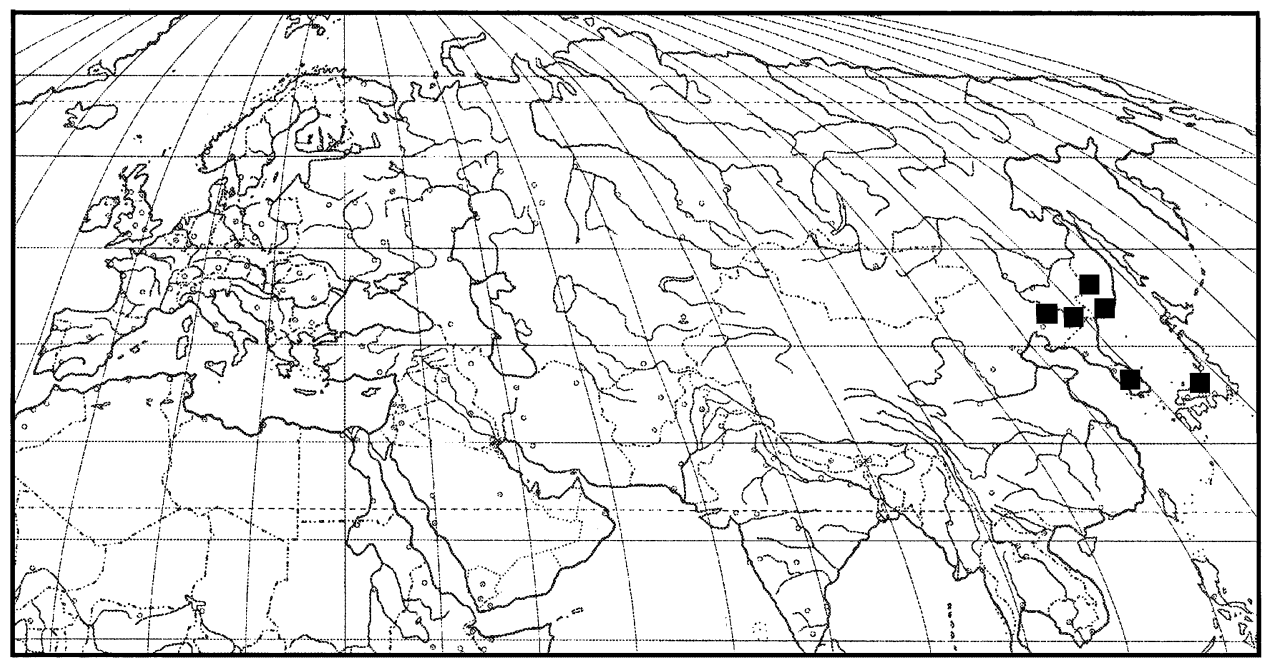

Map 1. Records of Ambanus. 
18. Ambanus terdecimus (Paik, 1978): female holotype from Waheyonri, Geoje-do, Kyungnam, Korea, in Kyungpook National University, Korea, not examined. NEW COMBINATION (transferred here from Coelotes).

\section{Ambanus mandzhuricus Ovtchinnikov Figures 57-63}

Ambanus mandzhuricus Ovtchinnikov, 1999: 63, figs. 1-5 (2 male and 2 female paratypes from Kedrovaya Pad Reservation, S-Primorie, far eastern Russia, in SZM, examined).

Diagnosis: This species is similar to $A$. paiki Ovtchinnikov but can be distinguished by the shape of copulatory ducts and the dorsally situated spermathecal heads of females (fig. 58) and by the different shape of the embolus and the broad conductor of males (figs. 59-61).

Description: Total length 10.0-12.0. From front, anterior eye row slightly procurved, posterior row procurved; eye sizes and arrangements: AME smallest, about half ALE size or slightly larger, ALE subequal to PLE or slightly larger, PME slightly smaller than PLE; AME-AME subequal to AMEALE, ALE-PLE close together, PLE-PME roughly PLE diameter, PME-PME roughly AME diameter (fig. 62). Clypeal height slightly more than twice AME diameter; chilum present, divided, elongated. Chelicerae with three promarginal and two retromarginal teeth (fig. 63). Labium slightly longer than wide. Length of female 1 st leg patella + tibia shorter than carapace length.

Tarsal organ and trichobothria not examined. Tarsal organ of Ambanus napolovi (from far eastern Russia) situated close to distal end of tarsus, anterior of distalmost trichobothrium (figs. 67-69).

Spinnerets not examined. Female spinnerets of $A$. napolovi: apex of ALS with 2 major ampullate gland spigots (MAP), 20 piriform gland spigots; PMS with at least 57 aciniform gland spigots, 2 cylindrical gland spigots (CY), and 1 or 2 minor ampullate gland spigots (mAP); PLE with at least 78 aciniform gland spigots and 3 cylindrical gland spigots (figs. 64-66). Spinnerets of A. rostratus also showed 3 cylindrical gland spigots on female PLS.

Female epigynum without epigynal teeth;
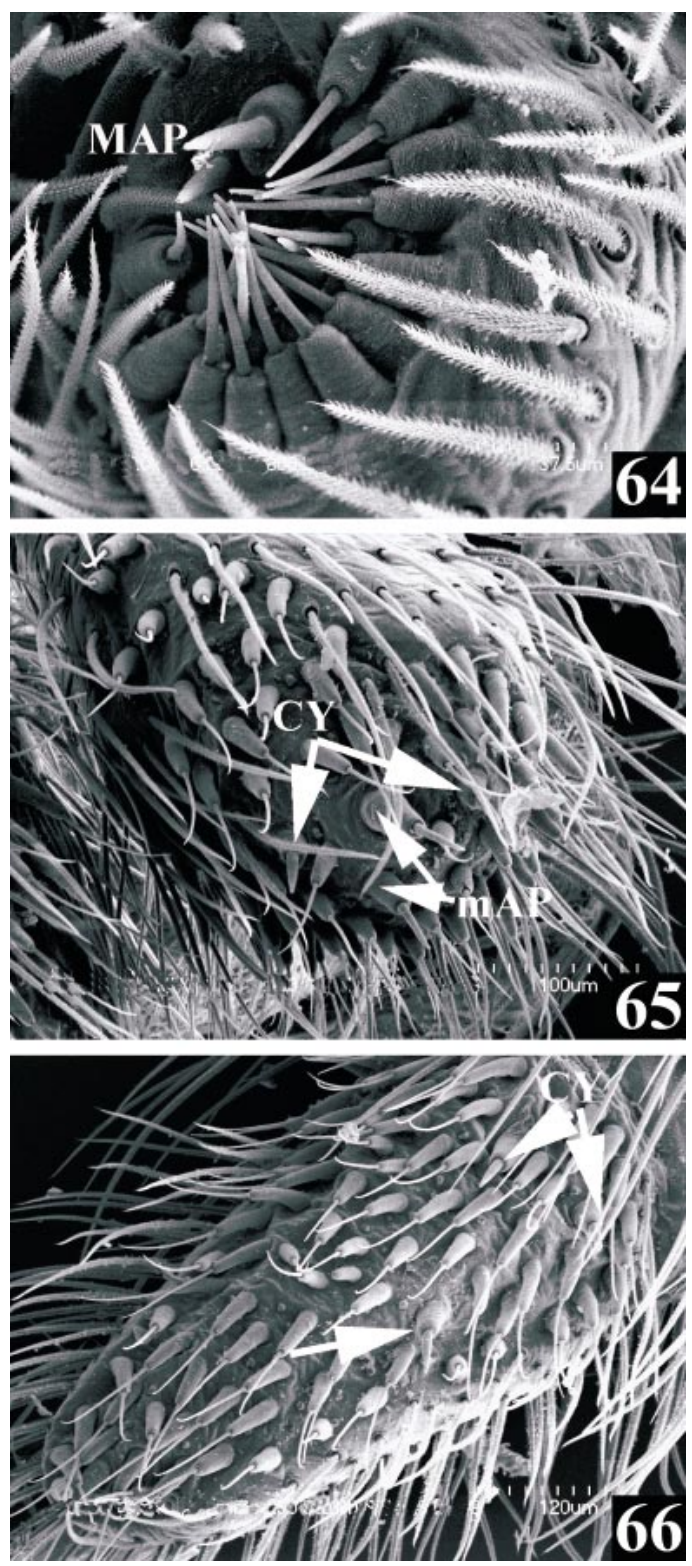

Figs. 64-66. Ambanus napolovi Ovtchinnikov, female spinnerets, ventral view, right. 64. ALS. 65. PMS. 66. PLS. Abbreviations are spelled out on p. 6 .

atrium large, deep; copulatory ducts broad; spermathecal heads small, situated laterad of spermathecae; spermathecae short, slightly convoluted, with indistinct stalks and bases (figs. 57, 58). Male palp with patellar apophysis absent; RTA moderately long, with dis- 



Figs. 67-69. Ambanus napolovi Ovtchinnikov, female. 67. Tarsus, showing position of tarsal organ (double arrows) and the distalmost trichobothrium (single arrow). 68. Tarsal organ. 69. Trichobothrium.

tal end slightly extended beyond tibia; lateral tibial apophysis modified to small concavity on distal tibia; cymbial furrow short, shallow, with dorsal edge not apparent; tegular sclerite more or less unsclerotized; conductor broad; conductor dorsal apophysis small; conductor lamella small; embolus basal in origin, strong, long, with spiral apex; median apophysis reduced (figs. 59-61).

MATERIal Examined: RUSSIA: Far East: S-Primorie, "Kedrovaya Pad" Reservation, August 31, 1978, 1 male and 1 female paratype (D. Sherbakov, SZM); S-Primorie, "Kedrovaya Pad" Reservation, June 27, 1976, 1 male and 1 female paratype (B. P. Zakharov, SZM).

\section{ASIACOELOTES, NEW GENUS}

Type Species: Coelotes xinhuiensis Chen, 1984.

ETyMOLOGY: The generic name derives from its similarity to Coelotes and distribution in East Asia, and is masculine in gender.

Diagnosis: This genus resembles Draconarius in having an elongated cymbial furrow, a long, slender embolus, and long, strongly convoluted spermathecae. Females can be distinguished by the broad, closely situated spermathecae (figs. 70, 71), males by the absence of the conductor dorsal apophysis (figs. 72-74).

DESCRIPTION: See description of type species (below). 2).

Distribution: China, Japan, Korea (map

Composition: Fifteen species are included, all are new combinations. Three new synonyms are recognized.

1. Asiacoelotes acco (Nishikawa, 1987): male holotype, male and female paratypes from Komori-ana Mine, Utobara, Futtsu-shi, Chiba, Japan, in NSMT and in the Arachnological Society of Japan, not examined. NEW COMBINATION (transferred here from Coelotes).

2. Asiacoelotes dicranatus (Wang et al., 1990): female holotype and male paratype from Jiangsu, China, in HBI, examined. NEW COMBINATION (transferred here from Coelotes).

The species Coelotes cinctus Zhu et al., 1993, with male and female paratypes from Dandong, Liaoning, China, in IZB, examined, is placed as a junior synonym of $A$. dicranatus (because of the identical male and female genitalic morphology). NEW SYNONYMY.

The species Coelotes paradicranatus Song 


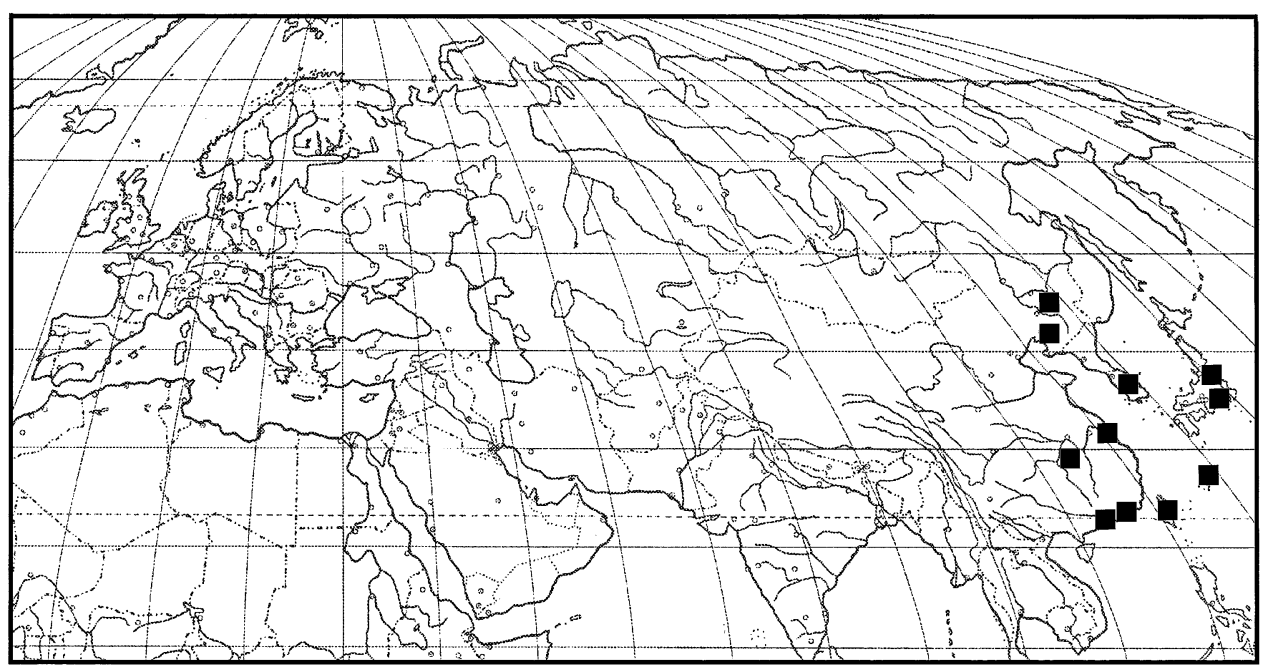

Map 2. Records of Asiacoelotes.

et al., 1993, with male paratypes from Qingyuan, Liaoning, in IZB, examined, is placed as a junior synonym of $A$. dicranatus (because of the identical male genitalic morphology). NEW SYNONYMY.

3. Asiacoelotes ensifer (Wang and Ono, 1998): male holotype and female paratype from Nantou, Taiwan, in NSMT, examined. NEW COMBINATION (transferred here from Coelotes).

4. Asiacoelotes illustratus (Wang et al., 1990): male and female paratypes from Changsha, Hunan, China, in HBI, examined. NEW COMBINATION (transferred here from Coelotes).

5. Asiacoelotes insidiosus (L. Koch, 1878): male holotype from Japan, depository unknown, not examined. Male and female specimens from Japan, in CAS, AMNH, and SMF, examined. NEW COMBINATION (transferred here from Coelotes).

The species Coelotes curtus (Bösenberg and Strand, 1906): female holotype from Saga, Japan, in SMF, examined, is placed as a junior synonym of $A$. insidiosus (L. Koch) (because of the identical female genitalic morphology). NEW SYNONYMY.

6. Asiacoelotes interunus (Nishikawa, 1977): male holotype, male and female paratypes from Minoo, Osaka, Japan, in the Osaka Natural History Museum and in the Arachnological Society of Japan, not examined. One male specimen from Japan, in
ZMB, examined. NEW COMBINATION (transferred here from Coelotes).

7. Asiacoelotes longus (Wang, Tso and Wu, 2001): male holotype from Nantou, Taiwan, in THU, examined. NEW COMBINATION (transferred here from Coelotes).

8. Asiacoelotes montivagus (Wang and Ono, 1998): male holotype and female paratype from Taichung, Taiwan, in NSMT, examined. NEW COMBINATION (transferred here from Coelotes).

9. Asiacoelotes pengi (Ovtchinnikov, 1999): female holotype from Yuelushan, Changsha, Hunan, China, in HBI, examined. NEW COMBINATION (transferred here from Coelotes). This species was initially described as Coelotes ovatus by Peng and Wang (1997), p. 330, and is a homomym of C. ovatus Paik, 1976.

10. Asiacoelotes songminjae (Paik and Yaginuma, 1969): female holotype from Yeongchi-gul Cave, Korea, in NSMT, not examined. Male and female specimens from Korea, in KAI and AMNH, examined. NEW COMBINATION (transferred here from Coelotes).

11. Asiacoelotes tengchihensis (Wang and Ono, 1998): female holotype from Mt. Tengchih, Tao-Yuan-hsinang, Kaohsiung, Taiwan, in NSMT, examined. NEW COMBINATION (transferred here from Coelotes).

12. Asiacoelotes tropidosatus (Wang and Zhu, 1991): female holotype from Changch- 


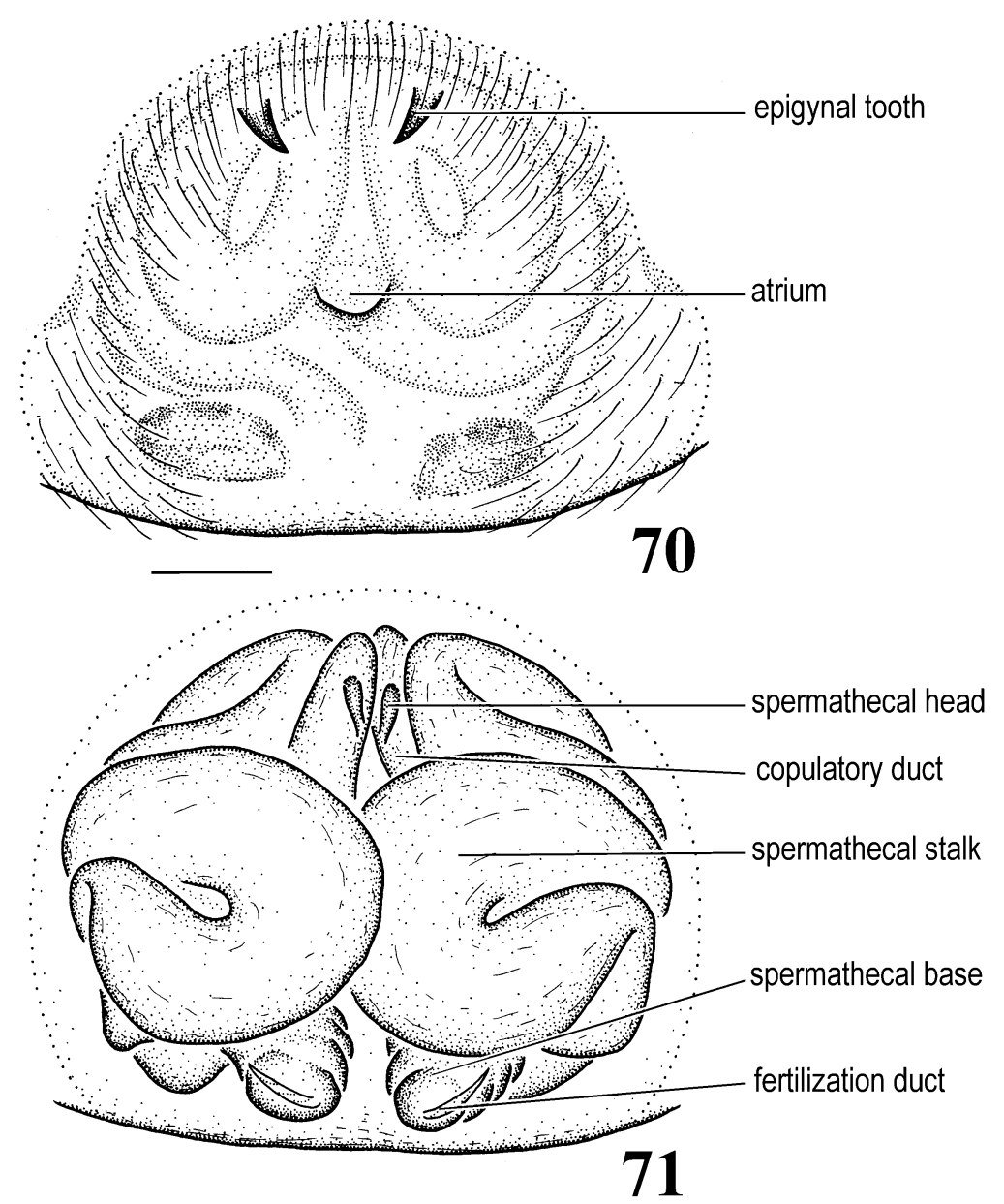

Figs. 70, 71. Asiacoelotes xinhuiensis (Chen), female. 70. Epigynum. 71. Vulva.

un, Jilin, China, in NBUMS, not examined. NEW COMBINATION (transferred here from Coelotes).

13. Asiacoelotes xinhuiensis (Chen, 1984): female holotype and female paratype from Xinhui, Guangdong, China, in HTC, examined. NEW COMBINATION (transferred here from Coelotes).

14. Asiacoelotes yaeyamensis (Shimojana, 1982): male holotype, male and female paratypes from Banna-dake, Ishigaki, Okinaea, Japan, in the Arachnological Society of Japan and in Shimojana's personal Collection, Japan, not examined. NEW COMBINATION (transferred here from Coelotes).

15. Asiacoelotes yushanensis (Wang and Ono, 1998): female holotype from Tatachia,
Nantou, in NSMT, examined. NEW COMBINATION (transferred here from Coelotes).

\section{Asiacoelotes xinhuiensis (Chen) \\ Figures 70-85}

Coelotes xinhuiensis Chen, 1984: 3, figs. 9, 10 (female holotype and female paratype from Xinhui, Guangdong, China, in HTC, examined). - Song et al., 1999: 78, figs. $216 \mathrm{~N}$, O, 226M, N.

Coelotes atratus Wang et al., 1990: 227, figs. 115, 116 (female holotype from Damingshan, Guangxi, China, in HBI, examined). First synonymized by Wang and Ono (1998).

Diagnosis: This species resembles $A$. songminjae (Paik and Yaginuma) (in Paik et al., 1969; Paik, 1971) but can be distin- 


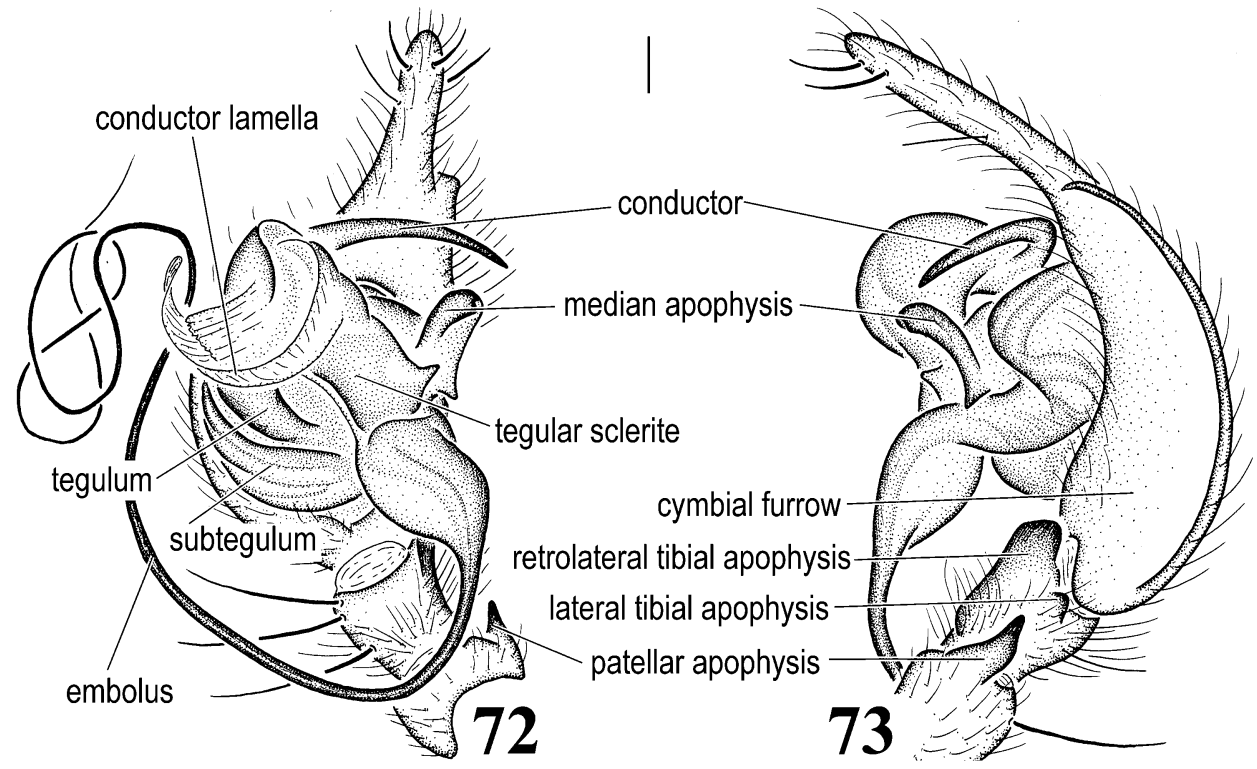

Figs. 72, 73. Asiacoelotes xinhuiensis (Chen), male palp. 72. Ventral view. 73. Retrolateral view.
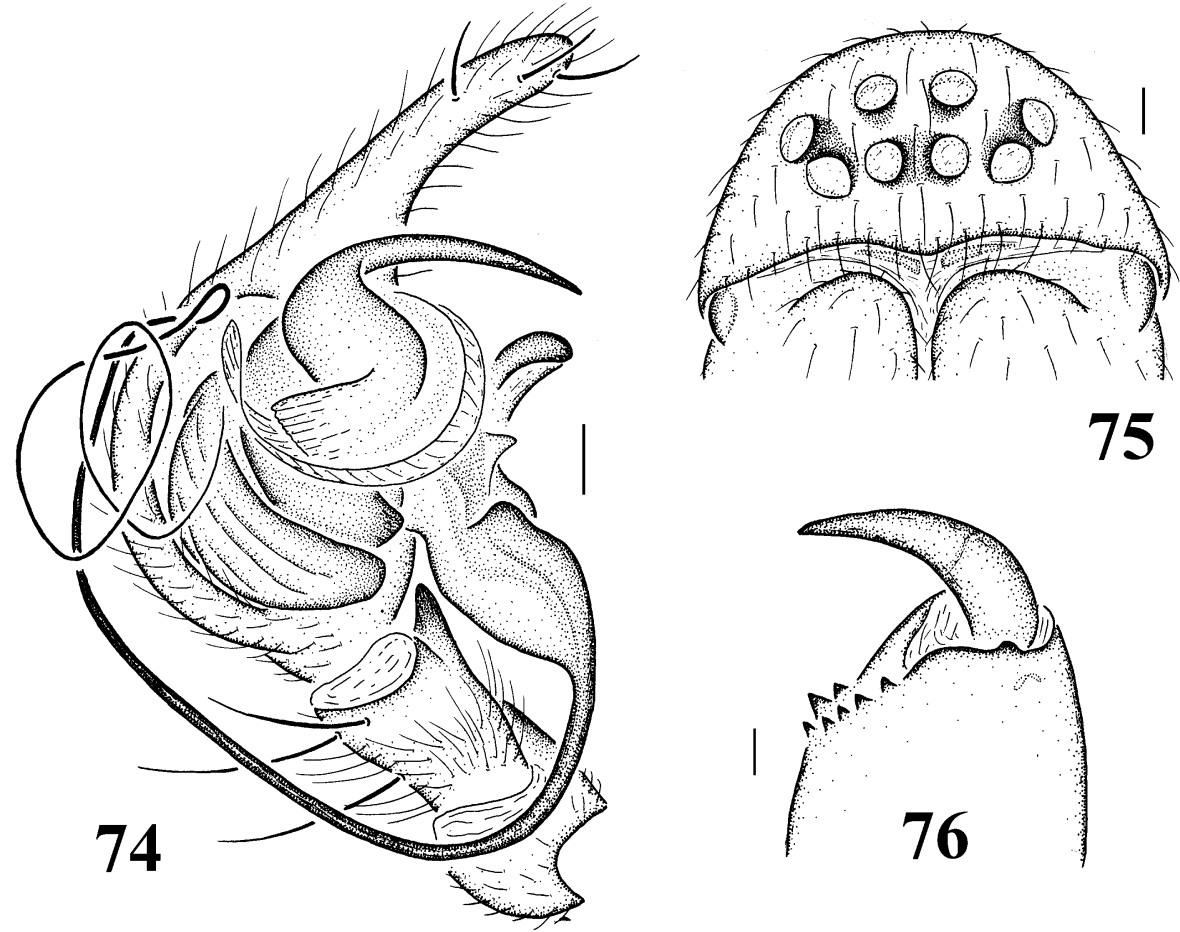

75

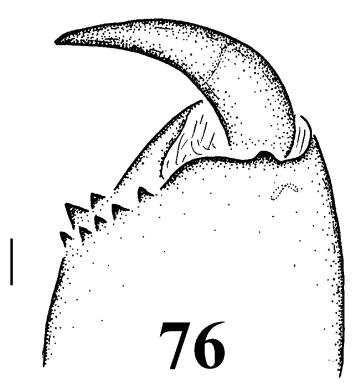

Figs. 74-76. Asiacoelotes xinhuiensis (Chen). 74. Male palp, prolateral view. 75. Eyes, dorsal view. 76. Chelicera, ventral view. 
guished by the distinct shape of spermathecae of females (figs. 70, 71) and by the presence of a small apophysis retrolaterally on the tegular sclerite of males (figs. 72, 73).

DESCRIPTION: Small, total length 4.508.00. From front, anterior eye row slightly procurved, posterior row procurved. Eye sizes and arrangements: AME slightly smaller than ALE, ALE subequal to PLE, PME subequal to AME or slightly larger than AME; AME separated by roughly their radius, AME-ALE and ALE-PLE close together, PME-PLE separated by roughly PLE diameter, PME-PME separated by roughly their diameter. Clypeal height about twice AME diameter or slightly less; chilum divided, elongated (fig. 75). Chelicerae with three promarginal, four retromarginal teeth (fig. 76). Labium slightly wider than long. Length of female 1st leg patella + tibia subequal to carapace length.

Tarsal organ situated close to distal end of tarsus, anterior of distalmost trichobothrium (figs. 77-79). Apex of ALS with 2 major ampullate gland spigots (MAP), about 28-33 piriform gland spigots (PI) in both sexes; PMS with 2 minor ampullate gland spigots (mAP) in both sexes, about 9-10 aciniform gland spigots (AC) in both sexes, 2 cylindrical gland spigots (CY) in female; PLS with about 13-16 aciniform gland spigots in both sexes, 2 cylindrical gland spigots in female (figs. 80-85).

Female epigynum with epigynal teeth short, anteriorly situated; atrium reduced to atrial slit; copulatory ducts short; spermathecal heads small; spermathecae with stalks strongly elongated, highly convoluted; spermathecal bases small (figs. 70, 71). Male palp with one patellar apophysis; RTA long, occupying almost tibial length, with distal end extended well beyond tibia; lateral tibial apophysis present; cymbial furrow strongly elongated, with distal end strongly extended beyond cymbium, dorsal edge strongly concave; conductor long, slender; conductor dorsal apophysis absent; conductor lamella large; embolus posterior in origin, extremely long, linear; median apophysis spoonlike, with distal end sharply pointed (figs. 72-74).

MATERIAL EXAMINED: CHINA: Guangdong: Xinhui, November 27, 1981, female holotype and female paratype (Z. H. Zhang,
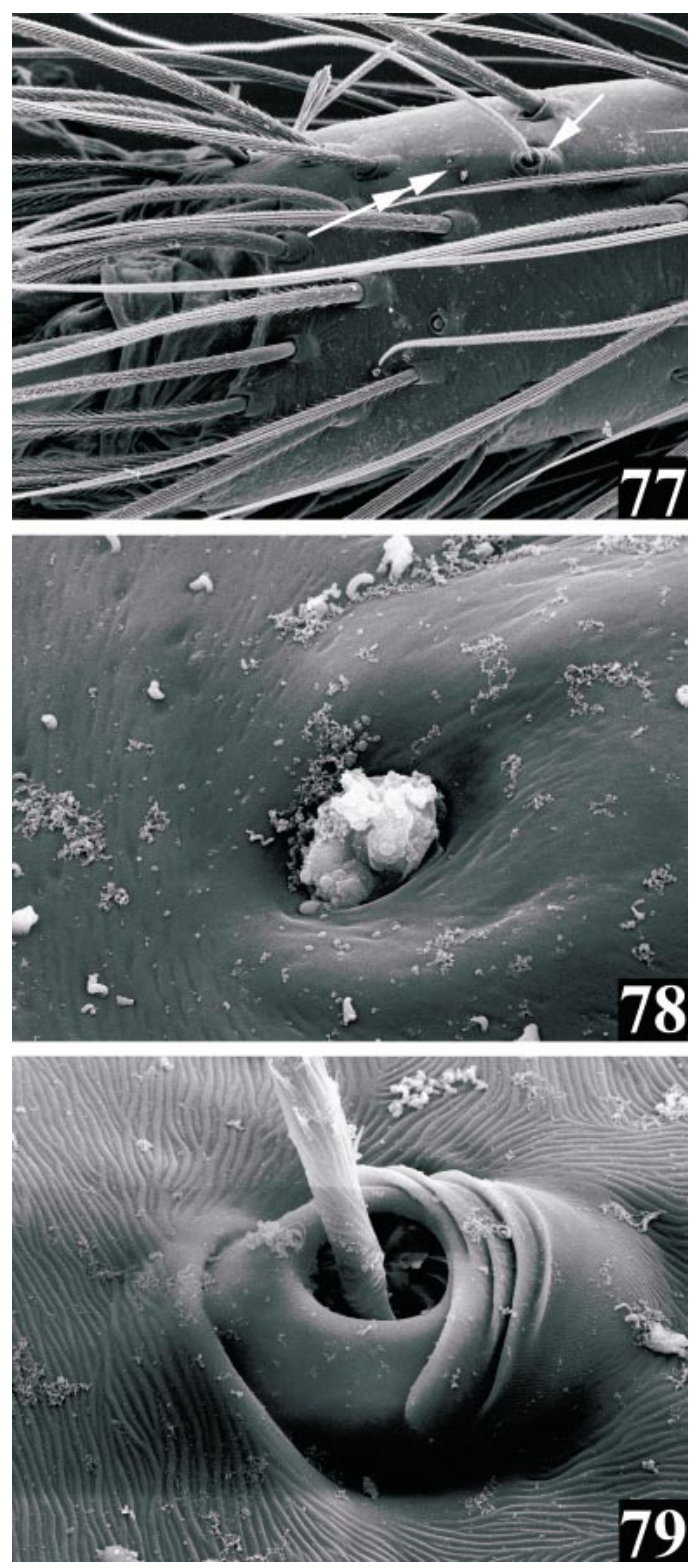

Figs. 77-79. Asiacoelotes xinhuiensis (Chen), female. 77. Tarsus, showing position of tarsal organ (double arrows) and the distalmost trichobothrium (single arrow). 78. Tarsal organ. 79. Trichobothrium.

HTC); Guangzhou, campus of the Southern China Teachers University, August 29, 1997, later molted to adult, 1 male (X. P. Wang, AMNH); Dinghushan, January 1982, 1 male (A. Payne, MCZ); Xiquio, January 1982, 1 male (A. Payne, MCZ). Guangxi: 

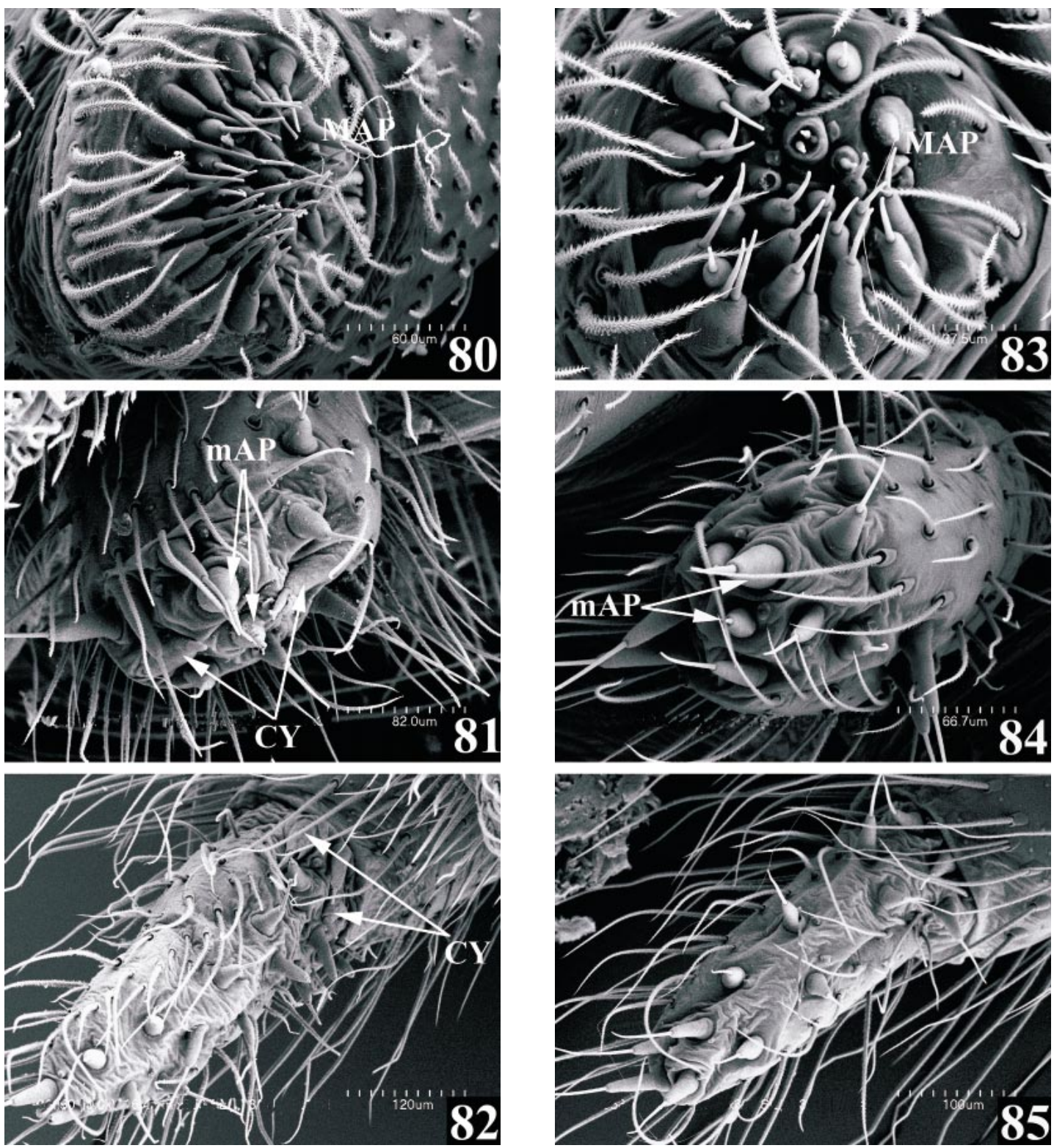

Figs. 80-82. Asiacoelotes xinhuiensis (Chen), female spinnerets, ventral view, left. 80. ALS. 81. PMS. 82. PLS. Abbreviations are spelled out on p. 6.

Figs. 83-85. Asiacoelotes xinhuiensis (Chen), male spinnerets, ventral view, left. 83. ALS. 84. PMS. 85. PLS. Abbreviations are spelled out on p. 6 .

Damingshan, August 10, 1982, female holotype of Coelotes atratus (J. F. Wang, HBI). Hong Kong: Tianpingshan, October 14, 1997, later molted to adults, 1 male and 3 females (X. P. Wang, 1 male and 2 females in HTU; 1 female in MCB). Taiwan: Tai-

pei, Quan In-shua, March 26, 1961, 1 female (E. Schlinger, AMNH); Nantou Hsien, Tatachia, $2480 \mathrm{~m}$ alt., March 4, 1991, 1 female (H. Ono, NSMT, NSMT-Ar.3424); Taichung Hsien, Mt. Chuanhsing-shan, $1590 \mathrm{~m}$ alt., March 2, 1991, 1 female (H. Ono, 


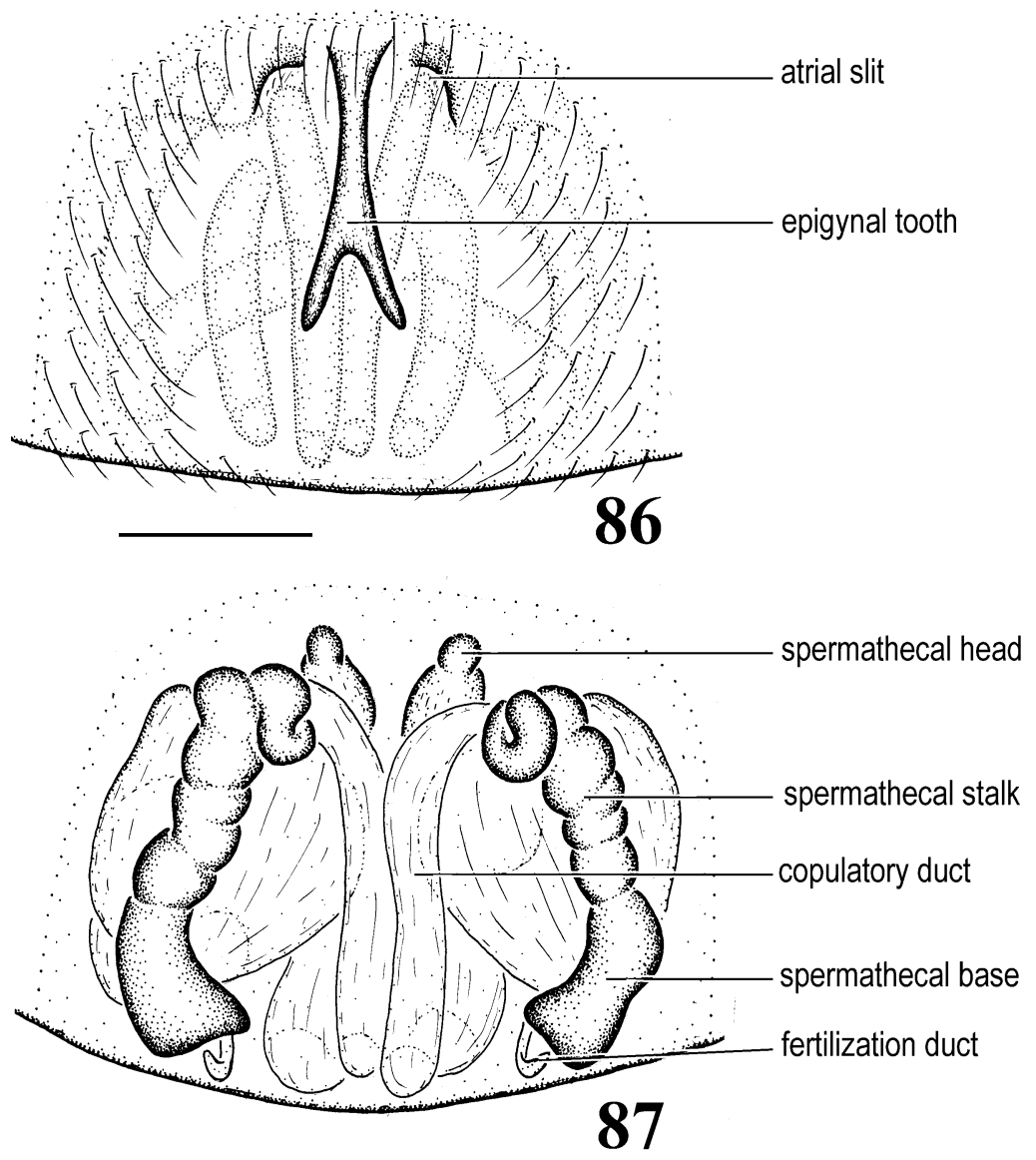

Figs. 86, 87. Bifidocoelotes bifida (Wang, Tso and Wu), female. 86. Epigynum. 87. Vulva.

NSMT, NSMT-Ar.3445); Kaohsiung Hsien, Taoyuan-hsiang, Paoshan-tsun, Mt. Tengchih, $1804 \mathrm{~m}$ alt., November 1, 1989, 1 male (H. Ono, NSMT, NSMT-Ar.3442); Taipei Hsien, Wulai, 300-400 $\mathrm{m}$ alt., November 27, 1989, 1 male (H. Ono, NSMT, NSMT-Ar.3452); Sozan, March 29, 1932, 1 male and 1 female (MCZ).

DisTRIBUTION: Southern China.

\section{BIFIDOCOELOTES, NEW GENUS}

TyPE SPECIES: Coelotes bifida Wang, Tso and $\mathrm{Wu}, 2001$

ETYMOLOGY: The generic name derives from its similarity to Coelotes and from the bifurcated epigynal teeth of females, and is masculine in gender.

DiAgnosis: Females can be easily recognized by their bifurcated epigynal teeth (fig.
86); males have a broad, bifid conductor and a small, rounded spoonlike median apophysis (figs. 88, 89). Both sexes have three promarginal and two retromarginal cheliceral teeth (fig. 91).

DESCRIPTION: See description of type species (below).

Distribution: China (map 3).

Composition: Two species are included. Both of them are new combinations.

1. Bifidocoelotes bifida (Wang, Tso and $\mathrm{Wu}, 2001)$ : female holotype and male paratype from Nantou, Taiwan, in THU, examined. NEW COMBINATION (transferred here from Coelotes).

2. Bifidocoelotes primus (Fox, 1937): female holotype and female paratype from Hong Kong, China, in AMNH and USNM, examined. NEW COMBINATION (transferred here from Coelotes). 


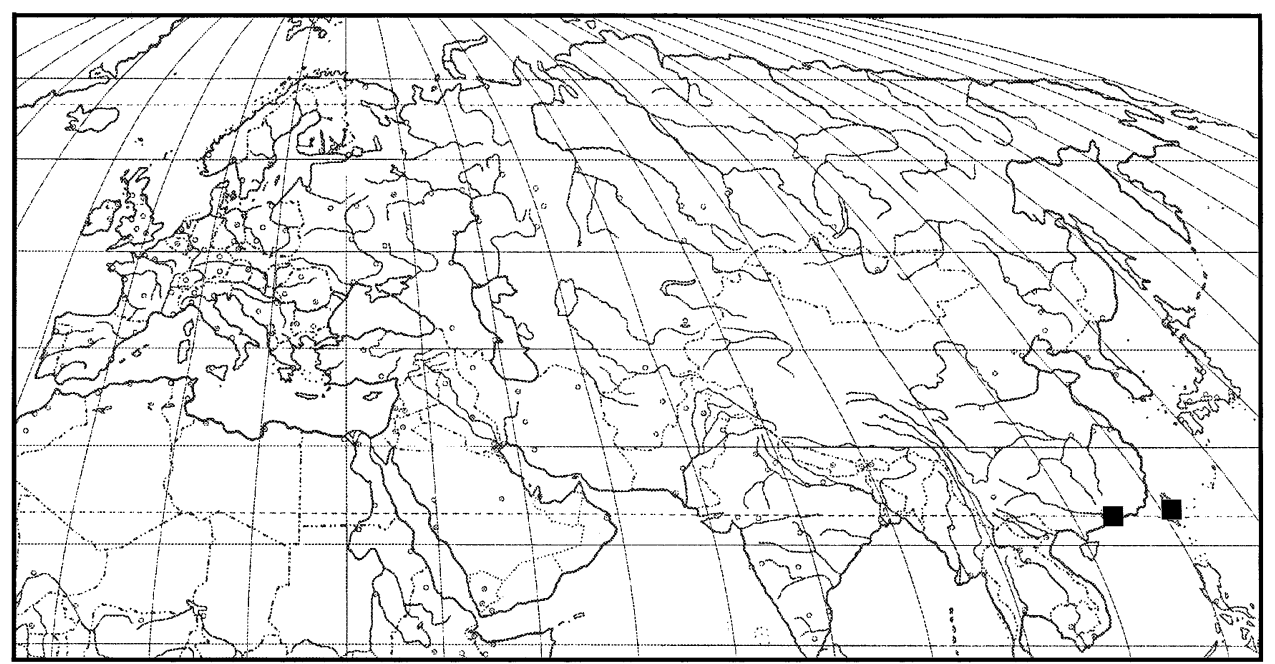

Map 3. Records of Bifidocoelotes.

Bifidocoelotes bifida

(Wang, Tso, and Wu, 2001)

Figures $86-100$

Coelotes bifida Wang, Tso and $\mathrm{Wu}, 2001:$ 128, figs. 1-4 (female holotype and male paratype from Nantou, Taiwan, in THU, examined).

Diagnosis: Females differ from $B$. primus by the anteriorly situated copulatory openings, the different shape of bifurcated epigynal teeth, the large copulatory ducts, the short spermathecal heads, and the longitudinally elongated spermathecae (figs. 86, 87), males have the broad, bifid conductor and small, rounded spoonlike median apophysis (figs. 88, 89).

Description: Total length 4.00-9.50. From front, anterior eye row slightly procurved, posterior row procurved; eye sizes and arrangements: AME smallest, about half ALE size or smaller, ALE largest, PLE slightly smaller than ALE, PME slightly smaller than PLE; AME-AME slightly shorter than their diameter, AME-ALE and ALEPLE close together, PME-PLE almost subequal to PME-PME or slightly longer, about size of PLE radius. Clypeal height slightly more than twice AME diameter; chilum divided, elongated (fig. 90). Chelicerae with three promarginal and two retromarginal teeth (fig. 91). Labium slightly shorter than wide. Length of female 1 st leg patella + tibia shorter than carapace length.
Tarsal organ situated close to distal end of tarsus, anterior of distalmost trichobothrium (figs. 98-100). Apex of ALS with 2 major ampullate gland spigots (MAP), 8-13 piriform gland spigots (PI) in both sexes; PMS with 7-10 aciniform gland spigots (AC) in both sexes, 2 cylindrical gland spigots (CY) in female, both sexes PMS with minor ampullate gland spigots (mAP) not apparent; PLE with 7-11 aciniform gland spigots in both sexes and 2 cylindrical gland spigots in female (figs. 92-97).

Female epigynum with single bifurcated epigynal teeth; atrium small, situated anteriorly; copulatory ducts large; spermathecal heads situated mesad of spermathecae; spermathecae elongated, convoluted, widely separated, with indistinct stalks and bases (figs. 86, 87). Male palp with large patellar apophysis; RTA long, with distal end broad, not extended beyond tibia; lateral tibial apophysis small; cymbial furrow long, with dorsal edge strongly concave and distal end slightly extend beyond cymbium; conductor strong, more or less spiral, with bifurcated apex; conductor dorsal edge with broad membranous extension; conductor dorsal apophysis small; conductor lamella large; embolus posterior in origin, very long; median apophysis spoonlike, small, with distal end smooth (figs. 88, 89).

Material Examined: CHINA: Taiwan: 



Figs. 88-91. Bifidocoelotes bifida (Wang, Tso and Wu). 88. Male palp, ventral view. 89. Male palp, retrolateral view. 90. Female, eyes, front view. 91. Female, chelicera, ventral view.

Nantou County, Huei-Sun Experimental Forest, elevation $1680 \mathrm{~m}$, March 31, 1998, female holotype (Hai-Yin Wu; THU, THU-Ar990017); Nantou County, Huei-Sun Experimental Forest, March 31, 1998, 1 male par- atype (Hai-Yin Wu; THU, THU-Ar-990020); Nantou County, Huei-Sun Experimental Forest, elevation $1680 \mathrm{~m}$, March 31, 1998, 1 male (Hai-Yin Wu, THU, THU-Ar-990019); Nantou County, Huei-Sun Experimental For- 

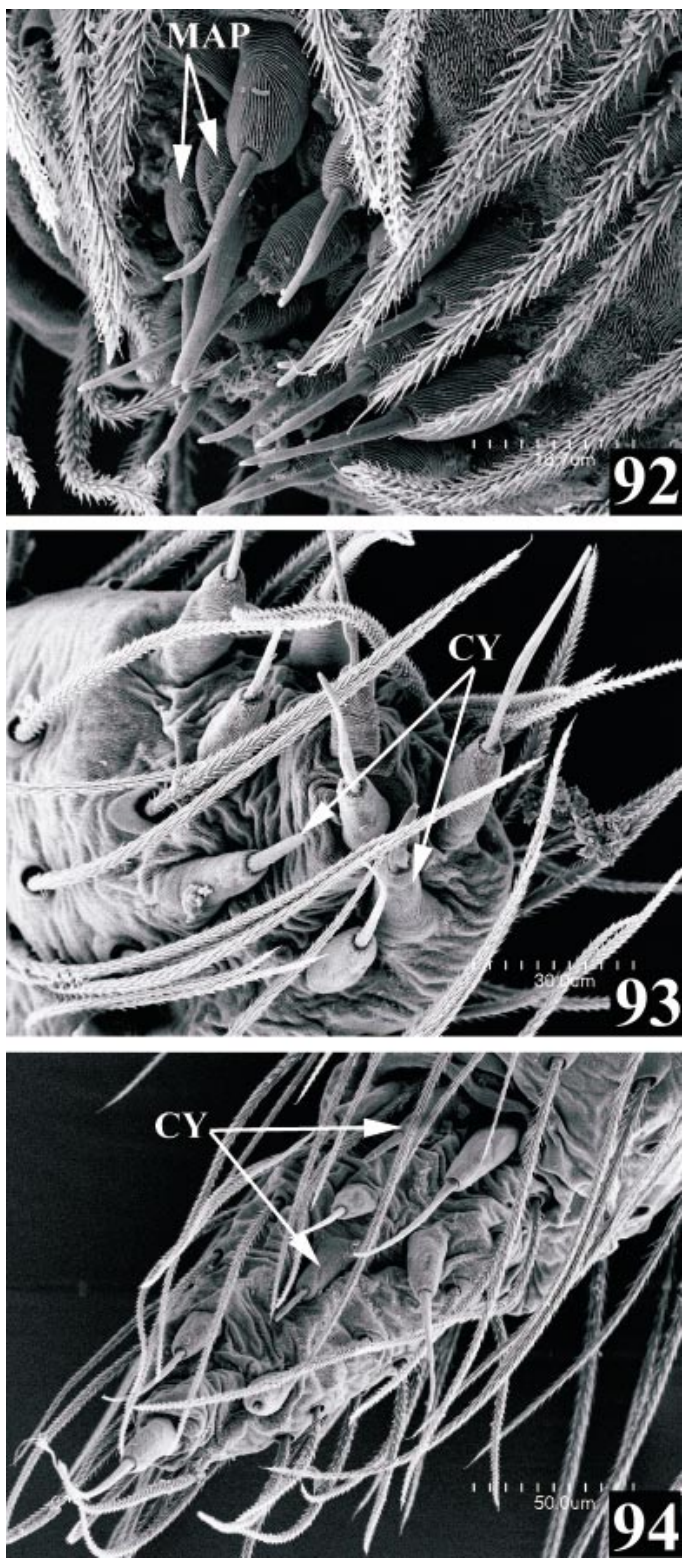

Figs. 92-94. Bifidocoelotes bifida (Wang, Tso and $\mathrm{Wu}$ ), female spinnerets, ventral view, right. 92. ALS. 93. PMS. 94. PLS. Abbreviations are spelled out on p. 6.

est, elevation $1675 \mathrm{~m}$, March 31, 1998, 1 male (Hai-Yin Wu, THU, THU-Ar-990026); Nantou County, Huei-Sun Experimental Forest, elevation $1550 \mathrm{~m}$, March 31, 1998, 1 female (Hai-Yin Wu, THU, THU-Ar-990027).

Distribution: China (Taiwan).

\section{COELOTES BLACKWALL}

Coelotes Blackwall, 1841: 618. - Roewer, 1954: 54; - Levi and Kraus, 1964: 150; - Lehtinen, 1967: 223; - Brignoli, 1983: 470; - Platnick, 1989: 421; - Platnick, 1993: 569; - Platnick, 1997: 662 .

TyPE SPECIES: Clubiona saxatilis Blackwall, 1833, by original designation, which is a junior synonym of Drassus atropos Walckenaer, 1830. Male neotype and female paraneotypes of $C$. atropos designated by de Blauwe (1973), deposited in Collection W. Kulczyn'ski, l'Institut de Zoologie P.A.N. de Varsovie, Poland, not examined.

DiAgNOSIS: Females resemble Eurocoelotes but have lateral epigynal teeth, a small atrium, and short copulatory ducts (figs. 38, 39, 101, 102, 113, 114, 127, 128). Males also resemble Eurocoelotes in having a conductor dorsal apophysis and a short, rounded median apophysis, but have a broad patellar apophysis (figs. 45, 107, 116, 130).

DESCRIPTION: See description of type species in Morphology section (above).

DisTRIBUTION: Europe, East Asia (map 4).

Composition: Four species groups are recognized within Coelotes: the atropos group, exitialis group, pseudoterrestris group, and charitonovi group, in total about 38 species.

In addition, 85 coelotine species are not well studied and temporarily remain in Coelotes (see appendix).

\section{The atropos Group}

DiAgNosis: Females have a reduced atrium, reduced copulatory ducts, and a short, less convoluted spermathecae (figs. 38, 39); males generally have a broad patellar apophysis, a small lateral tibial apophysis, a short conductor, and a broad conductor dorsal apophysis (figs. 44, 45).

This species group is well represented in Europe.

There are at least 19 species in the group. Some species, such as C. atropos (Walckenaer, 1830) and $C$. pastor Simon, 1875 , show remarkable intraspecific variation, particularly in female genitalia.

1. Coelotes alpinus Polenec, 1972: male holotype and female paratypes from Alps, Europe, in Prinodoelovni Muzej Slovenije, 

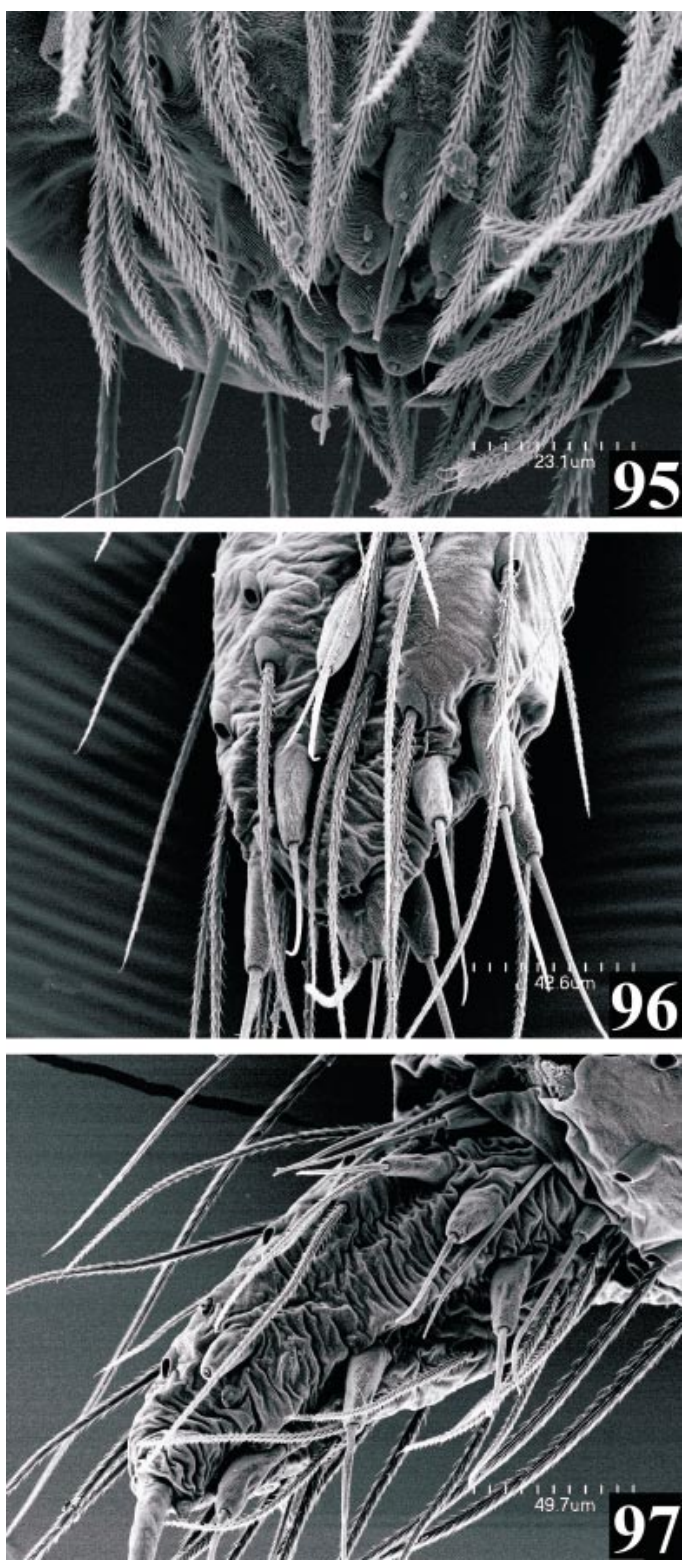

Figs. 95-97. Bifidocoelotes bifida (Wang, Tso and $\mathrm{Wu}$ ), male spinnerets, ventral view, right. 95. ALS. 96. PMS. 97. PLS.

Ljubljana, Slovenia, not examined. Male and female specimens from Italy, in MZF and $\mathrm{MCV}$, examined.

2. Coelotes atropos (Walckenaer, 1830): male neotype and female paraneotype from Europe, in the Collection W. Kulczyn'ski l'Institut de Zoologie P.A.N. de Varsovie, Po-
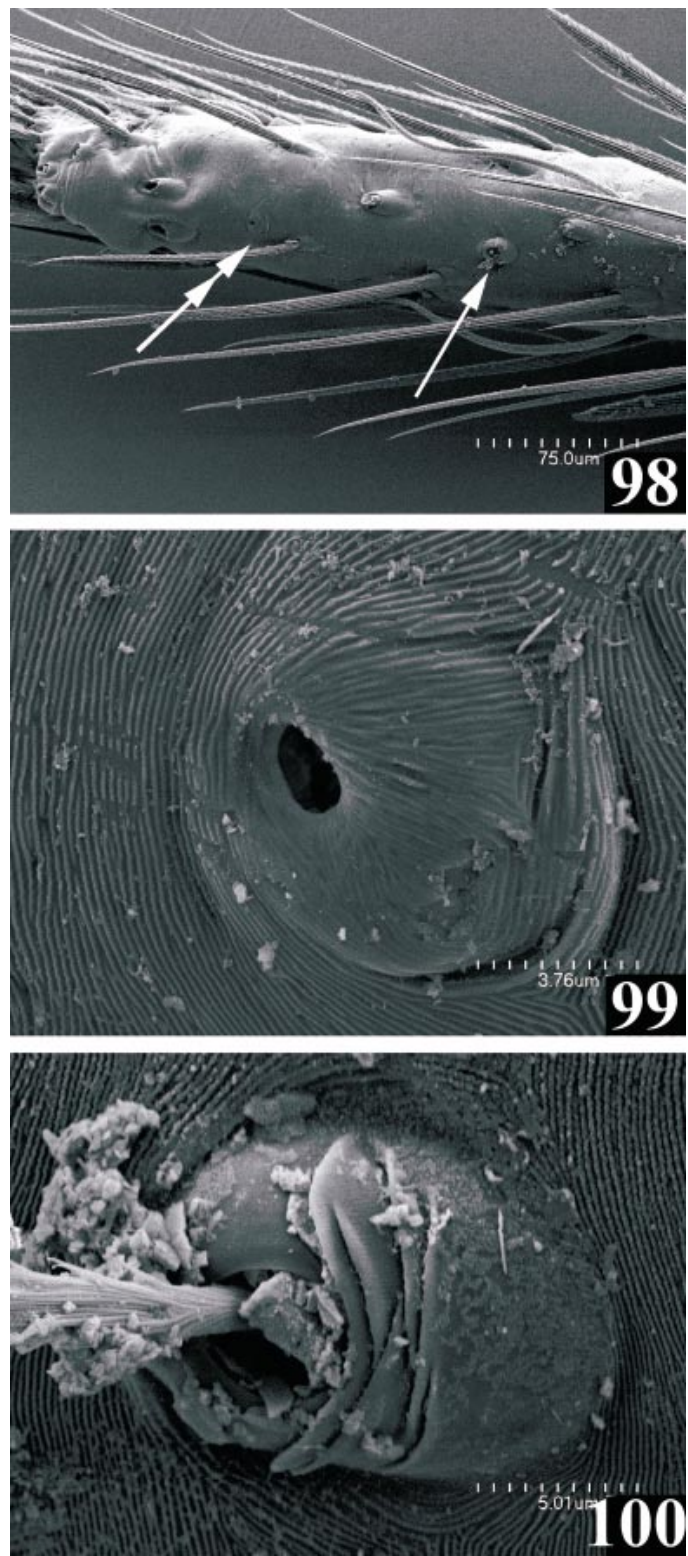

Figs. 98-100. Bifidocoelotes bifida (Wang, Tso and $\mathrm{Wu}$ ), female. 98. Tarsus, showing position of tarsal organ (double arrows) and the distalmost trichbothrium (single arrow). 99. Tarsal organ. 100. Trichobothrium.

land, not examined. Male and female specimens from England, France, Denmark, Poland, Switzerland, Russia, in CAS, AMNH, MCZ, NHMB, HEC, and COLL.WALTER, examined.

3. Coelotes atropos anomalus Hull, 1955: 


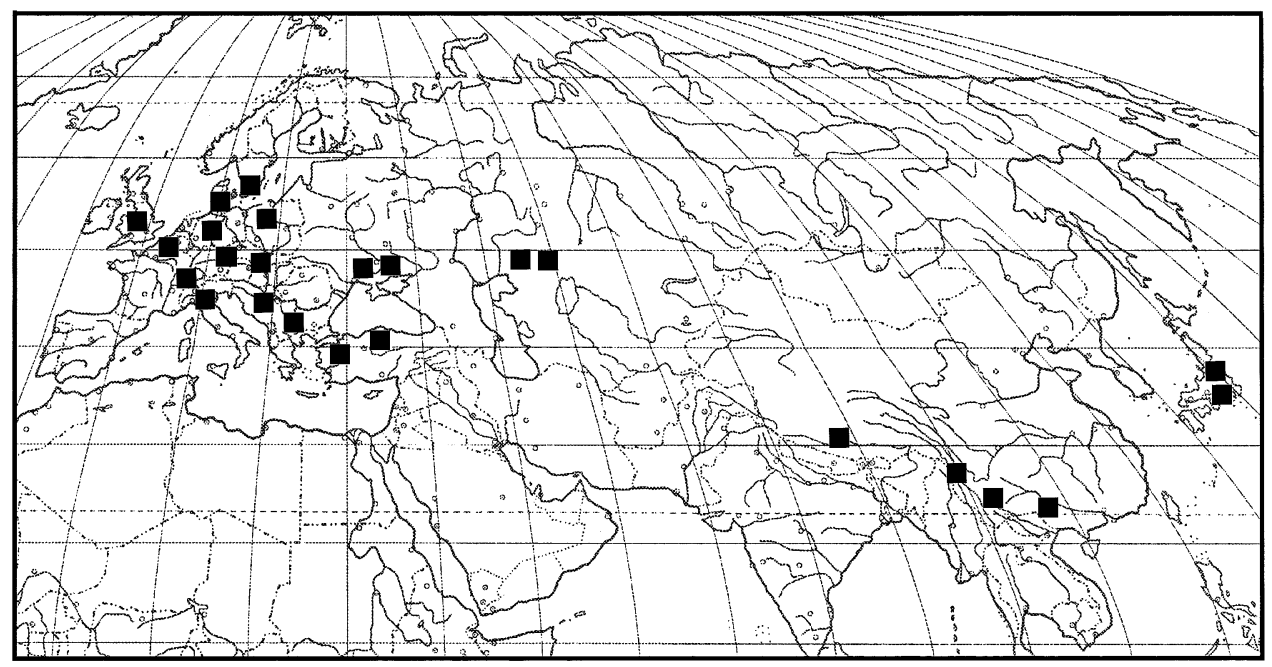

Map 4. Records of Coelotes.

female described from England, type not indicated and depository unknown, not examined. Judged by the illustration, this species may be a junior synonym of $C$. atropos. Further examination of possible type(s) is needed.

4. Coelotes atropus silvestris Hull, 1955: female described from England, type not indicated and depository unknown, not examined. Judged by the illustration, this species may be a junior synonym of $C$. atropos. Further examination of possible type(s) is needed.

5. Coelotes italicus Kritscher, 1956: 1 male paratype from Italy, in MCV, examined. Males and females from Italy, in MCV, $\mathrm{MCB}$, and AMNH, examined.

6. Coelotes mediocris Kulczyn'ski, 1887: type(s), depository unknown, not examined. Males and females from Italy, in NHMB, MCV, MCB, AMNH, IRSNB, COLL.MAURER, examined.

7. Coelotes osellai de Blauwe, 1973: male holotype, male and female paratypes from Italy, in MCV, examined.

8. Coelotes pabulator Simon, 1875: male lectotype and female paralectotype from France, in SMNH, examined.

9. Coelotes pastor Simon, 1875: male lectotype and female paralectotype from France, in MNHN, not examined. Males and females from France and Italy, in MNHN, MCZ, $\mathrm{MCV}$, and AMNH-CU examined.
10. Coelotes pastor carpathensis Ovtchinnikov, 1999: male holotype, male and female paratypes from Middle Asia, in CSO, not examined.

11. Coelotes pastor cooremani de Blauwe, 1975: male and female paratypes of C. cooremani de Blauwe from Italy, in MCV, examined. Maurer (1982) reduced the species C. cooremani de Blauwe, 1975 to this subspecies. Male types are C. rudolfi.

12. Coelotes pastor pirinicus Drenski, 1942: types unknown, not examined.

13. Coelotes pastor pickardi O.P.-Cambridge, 1873: types unknown, not examined. The species $C$. pickardi O.P.-Cambridge, 1873 was reduced to this subspecies by Maurer (1982). Males and females from Italy and Switzerland, in NHMB, COLL.MAURER, HEC, MCB, and AMNH examined.

14. Coelotes pastor tirolensis (Kulczyn'ski, 1906): types, unknown, not examined. Males and females from Switzerland, Italy, Austria, in NHMB, COLL.MAURER, $\mathrm{MCB}$, and MCV, examined.

The species Coelotes pastor lessinensis Maurer, 1982, with female holotype and male paratype from Italy, in $\mathrm{MCV}$, examined, is placed as a junior synonym of $C$. $p$. tirolensis (Kulczyn'ski) (because of the identical male and female genitalic morphology). NEW SYNONYMY.

15. Coelotes poleneci Wiehle, 1964: male holotype from Slovenia, in SMF, examined. 
16. Coelotes poweri Simon, 1875: female lectotype and male paralectotype from Italy, in MNHN, examined.

17. Coelotes rudolfi (Schenkel, 1925): male and female types from Switzerland, in NHMB, not examined. Male and female specimens from Switzerland, in AMNH and COLL.MAURER, examined.

18. Coelotes solitarius L. Koch, 1868: male neotype and female paraneotypes from Europe, in Collection W. Kulczyn'ski l'Institut de Zoologie P.A.N. de Varsovie, Poland, not examined. Females from Austria, Italy, Germany, in MCB, MZF, ZMB, and HEC, examined.

19. Coelotes terrestris (Wider, 1834): male neotype and female paraneotype from Europe, in Collection W. Kulczyn'ski l'Institut de Zoologie P.A.N. de Varsovie, Poland, not examined. Male and female specimens from Europe, in $A M N H$, COLL.MAURER, NHMB, HEC, MCZ, AMNH-CU, and ZMB, examined.

\section{Coelotes atropos (Walckenaer)} Figures 5-54

Drassus atropos Walckenaer, 1830: 170 (male neotype and female paraneotype from Europe, in the Collection W. Kulczyn'ski l'Institut de Zoologie P.A.N. de Varsovie, Poland, not examined). - Walckenaer, 1837: 627.

Drassus trucidator Walckenaer, 1830 (types unknown): 172. - Walckenaer, 1837: 629.

Clubiona saxatilis Blackwall, 1833 (types unknown): 436. - Blackwall, 1834: 332; - Blackwall, 1841: 618, figs. 6-8; - Walckenaer, 1847: 441; - Blackwall, 1861: 169, fig. 109.

Clubiona tigrina Walckenaer, 1842 (types unknown): 483.

Amaurobius atropos: Simon, 1864: 139. - Kulczyn'ski, 1908: 435, figs. 15, 28, 48, 66; Locket and Millidge, 1953: 19, figs. 14A, 14B; 15A, 15C; - Miller, 1971: 175, pl. XXVIII, figs. 20-22, pl. XXIX, fig. 18.

Amaurobius saxatilis: Simon, 1864: 139.

Amaurobius trucidator: Simon, 1864: 139.

Coelotes atropos: Simon, 1875: 32, fig. 14; Hansen, 1882: 42, fig. 2; - Becker, 1896: 187, fig. 2; - Chyzer and Kulczyn'ski, 1897: 160, fig. 15; - Bösenberg, 1902: 222, fig. 314. - Dahl, 1931: 22, figs. 34-41; - Reimoser, 1931: 40, fig. 2; - Simon, 1937: 985, 990, 1030, figs. 1511, 1520; - Drensky, 1942: 41, figs. 5c, 6cHull, 1955: 51, fig. 2e; - Wiehle, 1963: 289, figs. $107,110,112,117-119$; - Lehtinen, 1967:
448, fig. 239; - Loksa, 1969: 108, figs. 73B, 73C, 74B, 75E; - Tyschehenko, 1971: 160, figs. 448, 452; - de Blauwe, 1973: 27, figs. 24-28; -Locket et al., 1974: 43, fig. 23C; - Roberts, 1985: 164, fig. 73a; - Steinberger, 1989: 607, fig. 3; - Heimer and Nentwig, 1991: 356, fig. 927; - Roberts, 1995: 249; - Buchar et al., 1995: 120, figs. 35.12, 35.13 .

Coelotes saxatilis: Chrysanthus, 1965: 67, figs. 13, 8-9.

Diagnosis: This species is similar to $C$. terrestris but can be distinguished by the different shape of the spermathecae of females (fig. 39) and by the relatively large, rough conductor dorsal apophysis of males (fig. 44).

DESCRIPTION: See descriptions in Morphology section (above) and also by de Blauwe (1973).

MATERIAL EXAMINED: DENMARK: 1 male and 1 female (CAS). ENGLAND: Oxford Shire, 1 female; Lake District, 1 male (AMNH); Oxfordshire, Oxford, July 23, 1958, 1 female (H. and L. Levi, MCZ); Settle, West Riding Yorkshire, April, 1948, 3 females (B. Percy, AMNH); Yorkshire, July, 1948, 1 male (B. Malkin, AMNH); West Riding Yorkshire, April, 1948, 2 females (B. Percy, AMNH); Yorkshire, 2100 ft, July 9, 1948, 5 females (B. Alkin, AMNH); Wales, Lllangoed Castle, Breconshire, July 5, 1959, 3 females (B. Malkin, AMNH). FRANCE: 1 male and 1 female (MCZ); 1 female (MCZ); Paris, 1 male (J. H. Emerton, MCZ). POLAND: Babia Gora, mountain top, 1700 m, 1 female (B. Malkin and A. Kamocki, AMNH); Babia Gora, 1450 m, July 25, 1975, 1 male (B. Malkin and A. Kamocki, AMNH); Bialewieza, Narewka River, August 26, 1959, 4 males and 1 female (B. Malkin, AMNH); Bialewieza, Natl. Pk., September 23-26, 1982, 2 males and 5 females (B. Malkin, AMNH); Roztocze Natl. Pk., Bukowa Gora, June 20, 1987, 1 female (B. and H. Malkin, AMNH). RUSSIA: Biolower, 1 female (Ed. Reimoser, MCZ). SWITZERLAND: Zinal, Val d'Anniviers, unter Steinwall, 1690 m, July 19, 1979, 2 females (J. Walter, COLL. WALTER); Mase, Val d'Herens, Fichten-Larchen-Wald, 1900 m, July 16, 1980, 1 male (J. Walter, COLL. WALTER); Marchairuz, 1 male and 1 female (NHMB, 610a); 1 male and 1 female (HEC, B.437, t.45). 


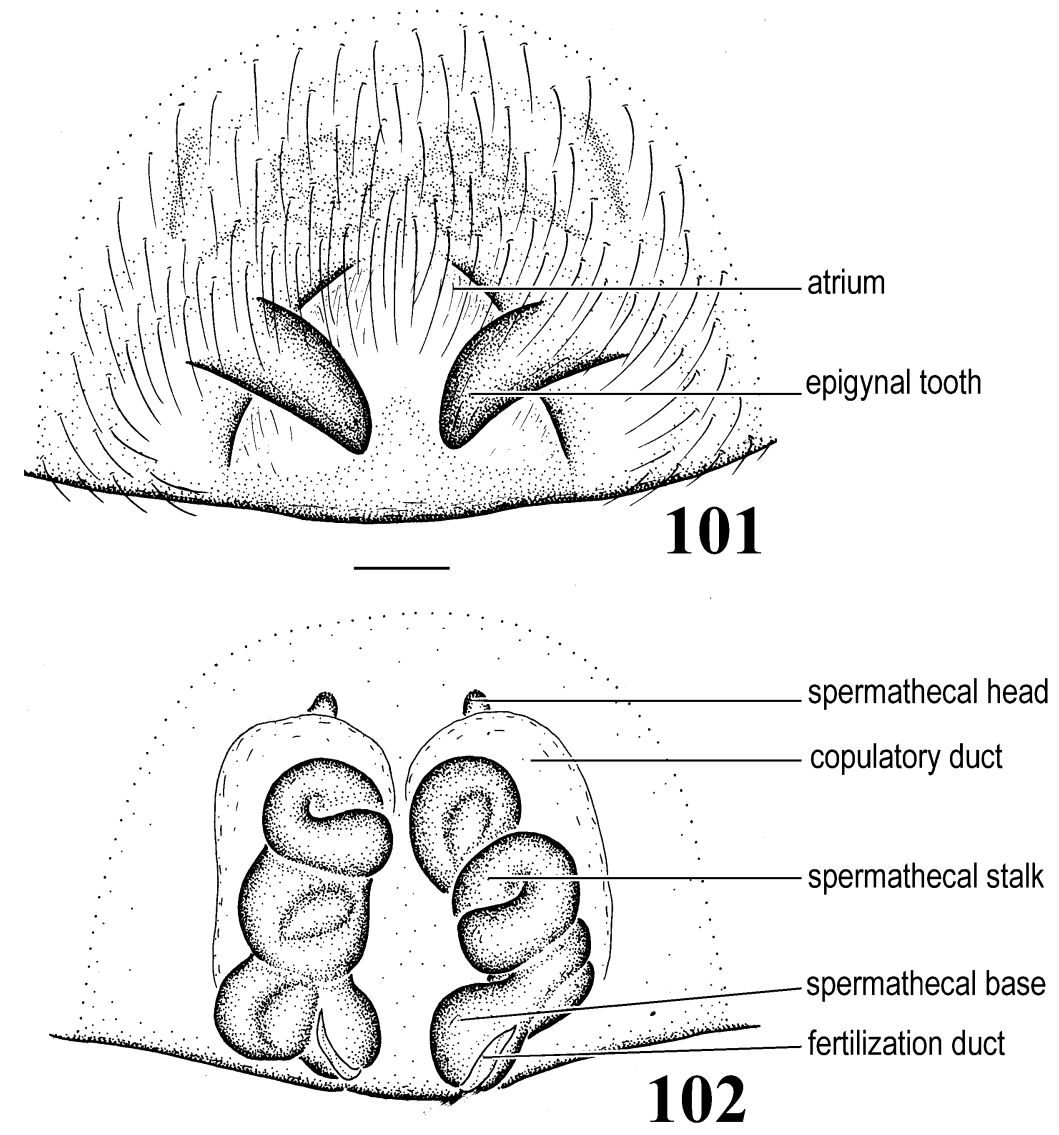

Figs. 101, 102. Coelotes exitialis Koch, female. 101. Epigynum. 102. Vulva.

The exitialis Group

DiAgNOSIS: Females have lateral atrial margins, broad copulatory ducts, and long, convoluted spermathecae (figs. 101-104); males generally have a well-developed lateral tibial apophysis, a long conductor, and a slender conductor dorsal apophysis (figs. 107-109).

This species group is well represented in Japan.

Nine species are here included in the group.

1. Coelotes antri (Komatsu, 1961): female holotype from Japan, depository unknown, not examined. Male and female from Shikoku, Japan, in AMNH, CAS, and SMF, examined.

2. Coelotes eharai Arita, 1976: male holotype, male and female paratypes from Mt. Daisen, Tottori, Japan, in the Ohtemon-Gak- uin University and in the Tottori Museum, Japan, not examined. A female from Shiko$\mathrm{ku}$, Japan, in AMNH, examined.

3. Coelotes exitialis L. Koch, 1878: male and female types from Japan, depository unknown, not examined. Male and female from Japan, in AMNH, CAS, and MCZ, examined.

4. Coelotes hiratsukai Arita, 1976: male holotype, male and female paratypes from Funaoka-cho, Tottori, Japan, in the Ohtemon-Gakuin University and in the Tottori Tottori Museum, Japan, not examined. One female from Shikoku, Japan, in AMNH, examined.

5. Coelotes kitazawai Yaginuma, 1972: female holotype from Fuji-san, Japan, depository unknown, not examined.

6. Coelotes micado Strand, 1907: types from Japan, depository unknown, not examined. 


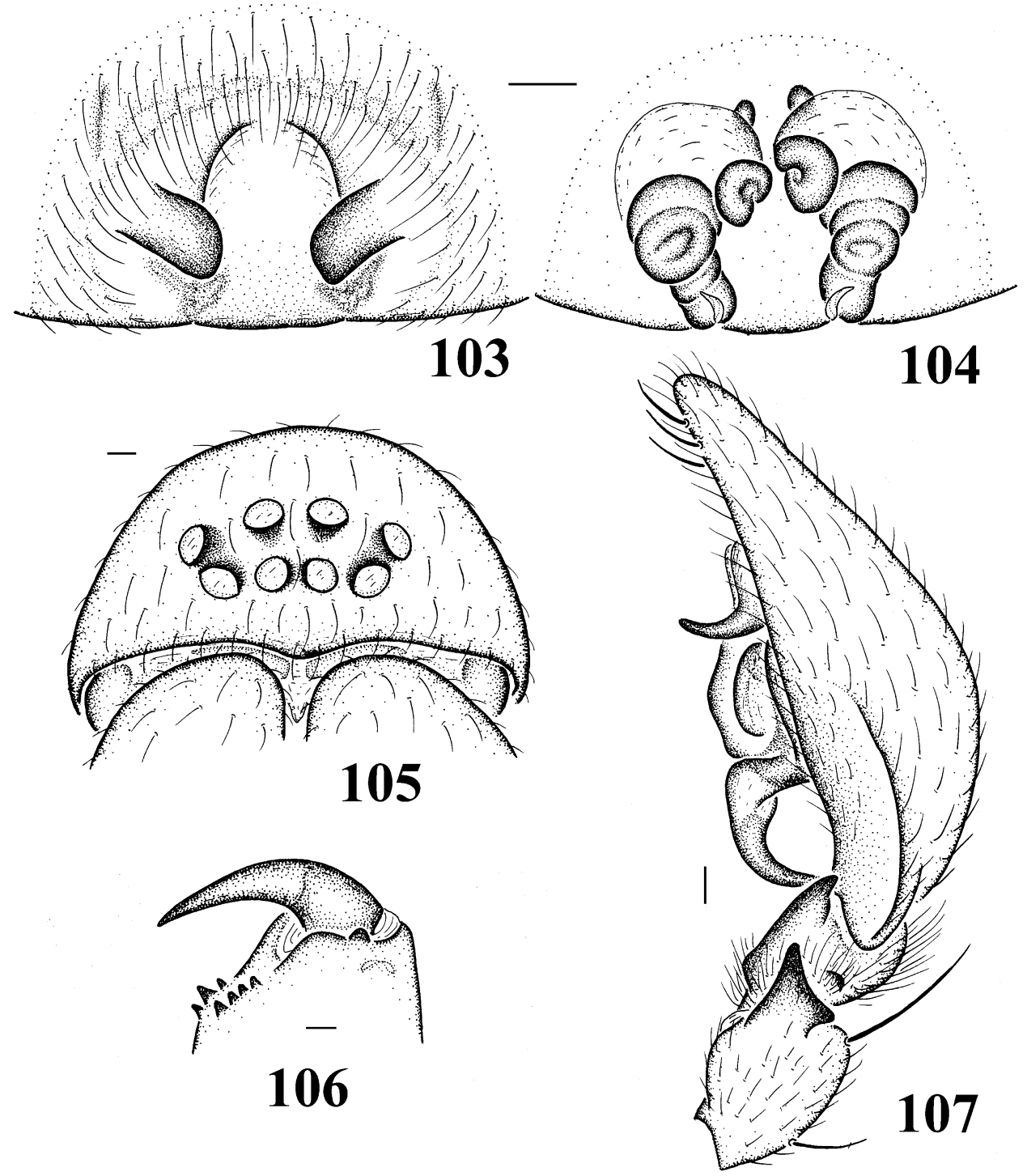

Figs. 103-107. Coelotes exitialis Koch. 103. Female, epigynum. 104. Female, vulva. 105. Carapace, front view. 106. Chelicera, ventral view. 107. Male palp, retrolateral view.

7. Coelotes musashiensis Nishikawa, 1989: female holotype, male and female paratypes from Tokyo, Japan, in the Arachnological Society of Japan and in NSMT, not examined. Two females from Japan, in ZMB, examined.

8. Coelotes yaginumai Nishikawa, 1972: female holotype and male paratype from Ibaraki, Osaka, Japan, in the Arachnological Society of Japan, not examined.

9. Coelotes yodoensis Nishikawa, 1977: male holotype, male and female paratypes from Minoo, Osaka, Japan, in the Arachnological Society of Japan and in the Osaka Natural History Museum, Japan, not examined.

\section{Coelotes exitialis L. Koch}

Figures 101-112

Coelotes exitialis L. Koch 1878: 755, figs.17-19 (types from Japan, depository unknown, not examined). - Uyemura, 1936: 10, figs. unnum- 


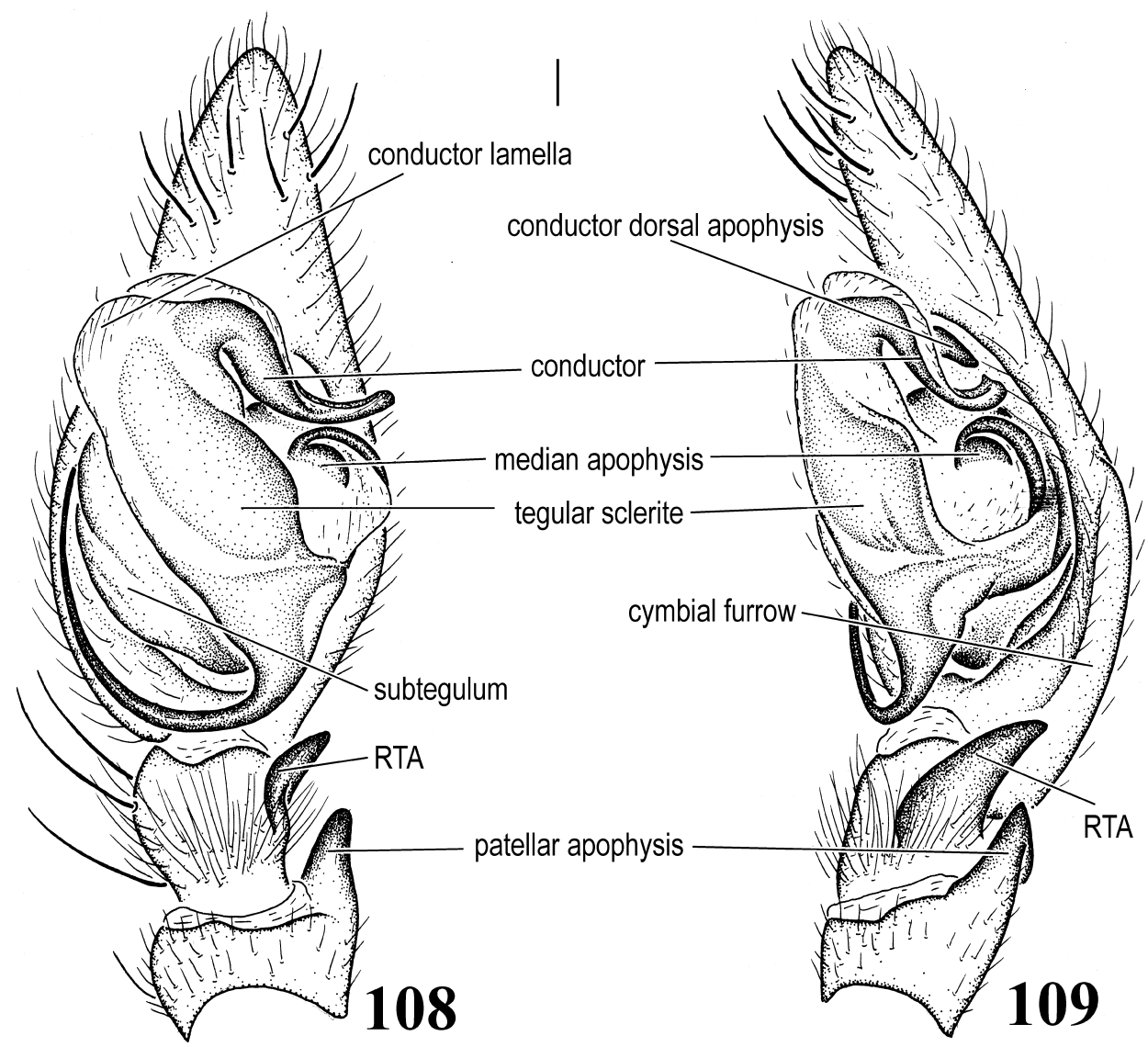

Figs. 108, 109. Coelotes exitialis Koch, male palp. 108. Ventral view. 109. Retrolateral view, showing conductor dorsal apophysis.

bered; - Yaginuma, 1960: 93, figs. 82.1, 82.2; - Yaginuma, 1971: 93, figs. 82.1, 82.2; - Yaginuma, 1973: 25, figs. 5, 6; - Nishikawa, 1974: 175, figs. 1, 2; - Nishikawa, 1975: 173, figs. 251; - Paik, 1978: 342, figs. 152.1, 152.2; - Yaginuma, 1986:147, fig. 79; - Chikuni, 1989: 100, fig. 11 .

Diagnosis: Females of this species can be recognized by the broad epigynal teeth and the anteriorly situated copulatory ducts (figs. $101,102)$, and males by the broad, slightly bifid patellar apophysis and the long, slender, slightly spiral conductor (figs. 107-109).

DESCRIPTION: See also description of Koch (1878). Total length 8.00-15.0. From front, anterior eye row slightly procurved, posterior row procurved; eye sizes and arrangements: AME smallest, ALE largest, PLE slightly smaller than ALE, PME subequal to PLE; AME-AME, AME-ALE, and ALE-PLE sub- equal or AME-ALE slightly longer, PLEPME wider than PLE diameter, PME-PME slightly wider than AME-AME. Clypeal height slightly more than twice AME diameter; chilum divided, elongated (fig. 105). Chelicerae with three promarginal and four retromarginal teeth (fig. 106). Labium slightly longer than wide. Length of female 1 st leg patella + tibia shorter than carapace length.

Tarsal organ situated close to distal end of tarsus, slightly anterior of distalmost trichobothrium. Only female spinnerets examined: apex of ALS with 2 major ampullate gland spigots (MAP), 45-50 piriform gland spigots; PMS with about 47 aciniform gland spigots, 2 cylindrical gland spigots (CY), 1 or 2 minor ampullate gland spigots (mAP); PLS with 56 aciniform gland spigots and 2 cylindrical gland spigots (figs. 110-112). 
Female epigynum with epigynal teeth broad, may vary in shape and position (Nishikawa, 1974); atrium reduced, with more or less apparent lateral atrial margins; copulatory ducts broad; spermathecal heads situated anteriorly; spermathecae elongated, convoluted (figs. 101-104). Male palp with patellar apophysis large, slightly bifid, may vary within species (Nishikawa, 1974); RTA long, with distal end sharply extended beyond tibia; lateral tibial apophysis present; cymbial furrow short; conductor long, more or less spiral, slender, its shape may vary within species (Nishikawa, 1974); conductor dorsal apophysis long; conductor lamella moderately developed; embolus basal origin, moderately long; median apophysis spoonlike, rounded, with distal end smooth (figs. 107-109).

Material ExAmined: JAPAN: Kobu (Kobe?), February 1909, 1 female (together with a male P. luctuosus) (S. C. Thompson, CAS); Shizuoka Pref., Izu Penninsula, 100400 m, August 10. 1962, 1 male (K. Sekiguchi and A. Ogura, MCZ); Fuji Mt., SE slope of Mt. Fuji, February 18, 1955, 2300 $\mathrm{m}$, floor mat., 1 female (D. C. Lowrie, AMNH).

DiSTRIBUTION: Japan.

\section{The charitonovi Group}

DiAGNOSIS: Females of this group have the epigynal teeth situated anteriorly, close to each other (fig. 113); males have the strongly modified, broad patellar apophysis (figs. 115-117).

This species group is well represented in Middle Asia.

There are 8 species in the charitonovi group.

1. Coelotes arganoi Brignoli, 1978: female holotype and paratypes from Erzincan, Turkey, in MHNG and MCV, examined.

2. Coelotes caudatus de Blauwe, 1973: female holotype and paratypes from Liban (Lebanon), in MNHN, examined.

3. Coelotes charitonovi Spassky, 1939: female holotype from Uzbekistan, depository unknown, not examined. One male and $1 \mathrm{fe}-$ male from Tadzhikistan, in AMNH, examined.

4. Coelotes coenobita Brignoli, 1978: fe-
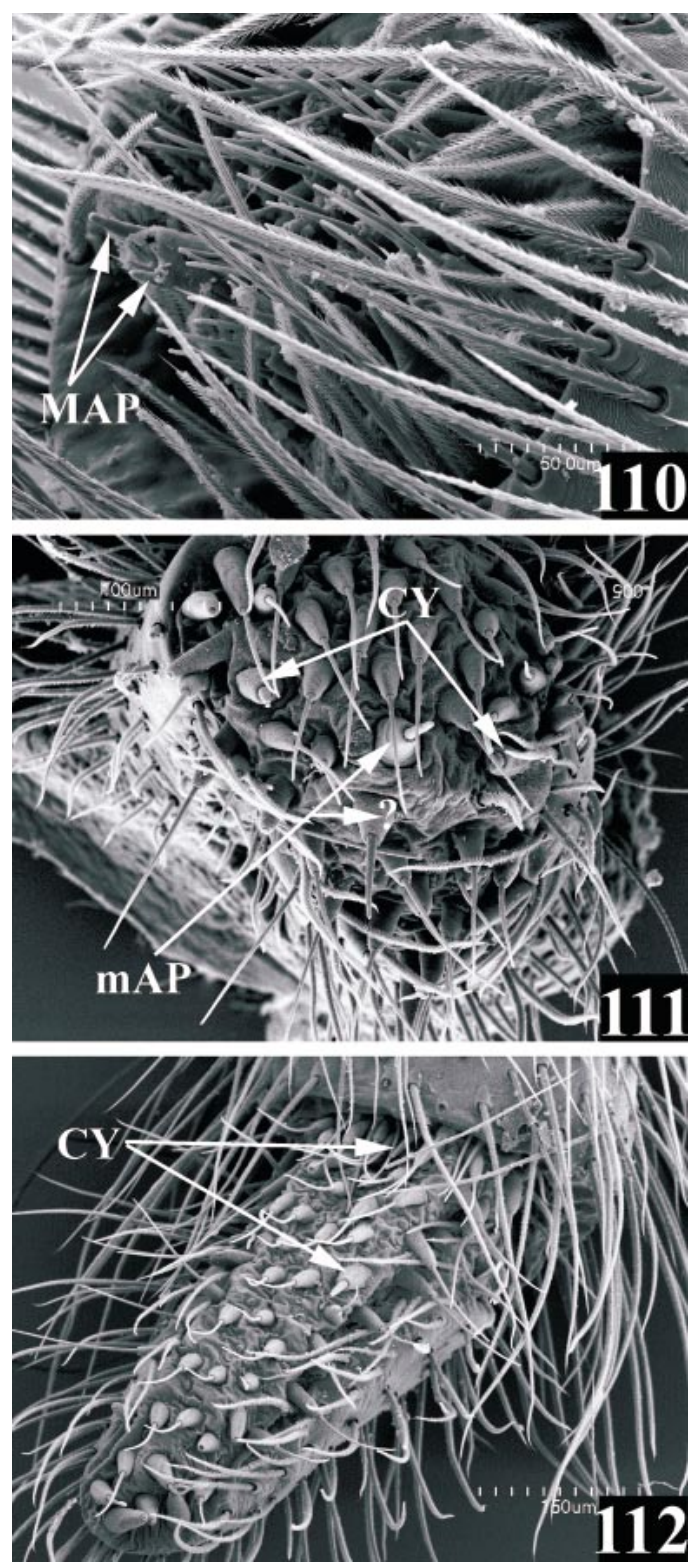

Figs. 110-112. Coelotes exitialis Koch, female spinnerets, ventral view, right. 110. ALS. 111. PMS. 112. PLS. Abbreviations are spelled out on p. 6.

male holotype and paratypes from Sumela (Macka), Trabzon, Turkey, in MHNG and MCV, examined.

5. Coelotes juglandicola Ovtchinnikov, 1984: male holotype, male and female paratypes from Kizghizia, depository unknown, 


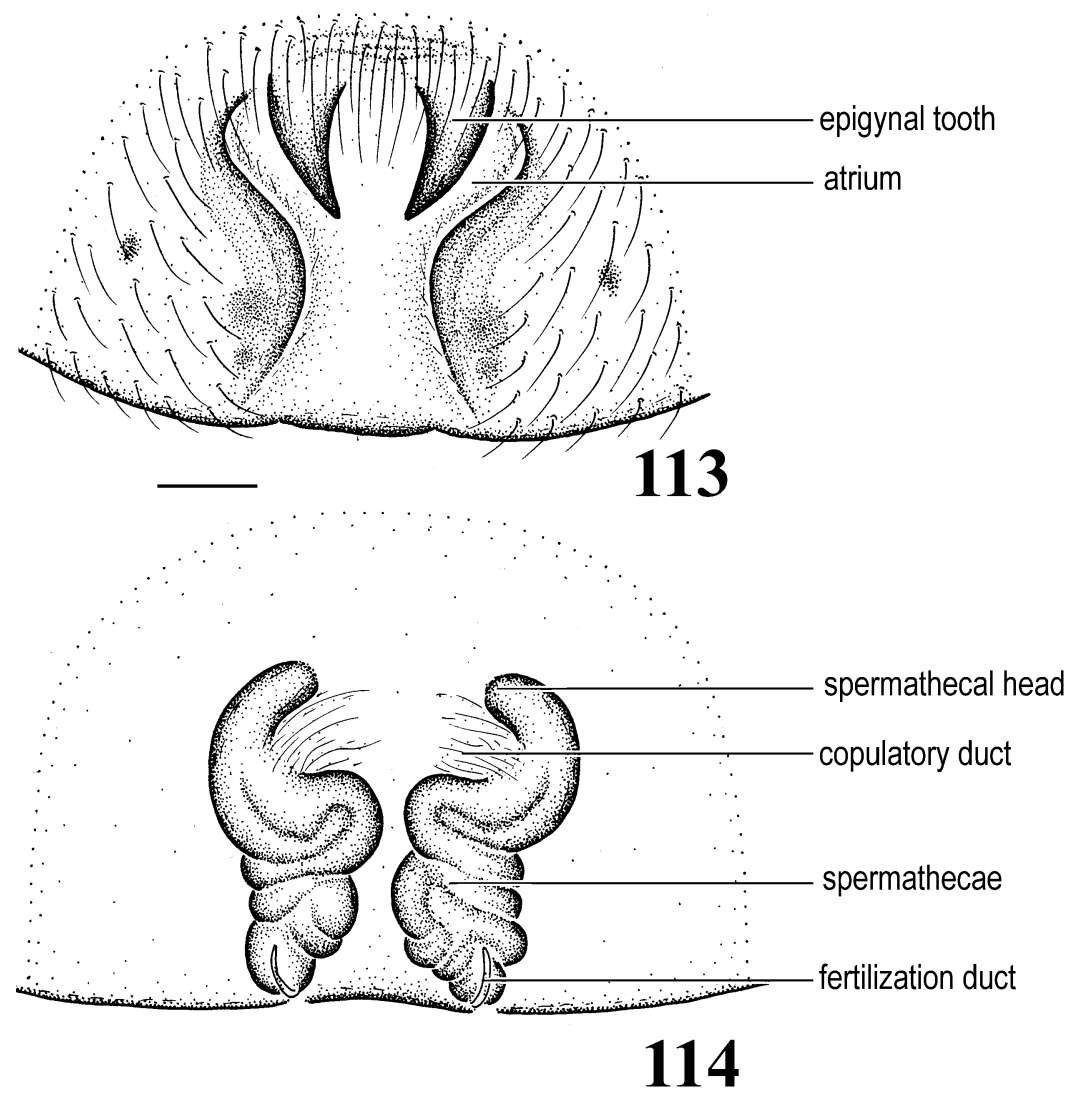

Figs. 113, 114. Coelotes charitonovi Spassky, female. 113. Epigynum. 114. Vulva.

not examined. One male and 1 female from Kizghizia, in AMNH, examined.

6 Coelotes nenilini Ovtchinnikov, 1999: male holotype, male and female paratypes from Kazakhstan, in CSO, not examined.

7. Coelotes turkestanicus Ovchinnikov, 1999: male holotype, male and female paratypes from Kirgizstan, in AMNH, examined.

8. Coelotes vignai Brignoli, 1978: female holotype and paratypes from Abant, Bolu, Turkey, in MNHG, examined.

\section{Coelotes charitonovi Spassky \\ Figures 113-126}

Coelotes charitonovi Spassky, 1939: 141, fig. 4 (types from Uzbekistan, depository unknown, not examined). - Ovtsharenko and Fet, 1980: 446; - Ovtchinnikov, 1999: 74, figs. 34, 35.

Agelena bucharensis Charitonov, 1969: 81, fig. 3. First synonymized by Ovtsharenko and Fet, 1980: 446.
Coelotes bucharensis: Ovchinnikov, 1988: 141.

Diagnosis: This species is similar to $C$. juglandicola, but females can be distinguished by the closely situated epigynal teeth and the broad, anteriorly situated spermathecal heads (figs. 113, 114), males by the different shape of conductor and the small median apophysis (figs. 115-117).

DESCRIPTION: See also Spassky (1939). From front, anterior eye row slightly procurved, posterior row also slightly procurved; eye sizes and arrangements: ALE largest, AME, PLE, and PME subequal or PME slightly smaller; AME-AME about twothirds AME diameter, AME-ALE and ALEPLE close together, PLE-PME subequal to PME-PME. Clypeal height roughly 1.5 times AME diameter; chilum divided, elongated (fig. 120). Chelicerae with five promarginal and five retromarginal teeth (fig. 119). La- 




Figs. 115, 116. Coelotes charitonovi Spassky, male palp. 115. Ventral view. 116. Retrolateral view.

bium shorter than wide. Length of female 1st leg patella + tibia longer than carapace length.

Tarsal organ situated close to distal end of tarsus, slightly anterior of distalmost trichobothrium (figs. 121-123). Female spinnerets with apex of ALS with 2 major ampullate gland spigots (MAP), 13 piriform gland spigots (PI); PMS with roughly 27 aciniform gland spigots, 2 cylindrical gland spigots (CY), 1 reduced ampullate gland spigot (mAP); PLE with 34 aciniform gland spigots and 2 cylindrical gland spigots (figs. 124126).

Epigynum with epigynal teeth large, situated anterad of atrium, close together; atrium reduced; copulatory ducts short, situated mesad of spermathecae; spermathecal heads large, anteriorly situated; spermathecae broad, with stalks and bases indistinct (figs. $113,114)$. Male palpal patellar apophysis broad, strongly modified into deep concavity and apophyses on inner side; RTA long, with distal end strongly extend beyond tibia; lateral tibial apophysis reduced; cymbial furrow short; conductor short; conductor dorsal apophysis broad; embolus prolateral in origin, moderately long, linear; median apophysis small, situated relatively posteriorly (figs. 115-117).

MATERIAL EXAMINED: TADZHIKISTAN: Near Khovaling town, $1000 \mathrm{~m}$ alt., November 7, 1987, 1 male and 1 female (S. Ovchinnikov, AMNH-donation of S. Ovtchnnikov).

DisTRIBUTION: Tadzhikistan.

\section{The pseudoterrestris Group}

DiAGNOSIS. Females of this group have a distinct anterior atrial margin, broad copulatory ducts, and long, convoluted spermathecae (figs. 127, 128); males generally have a 

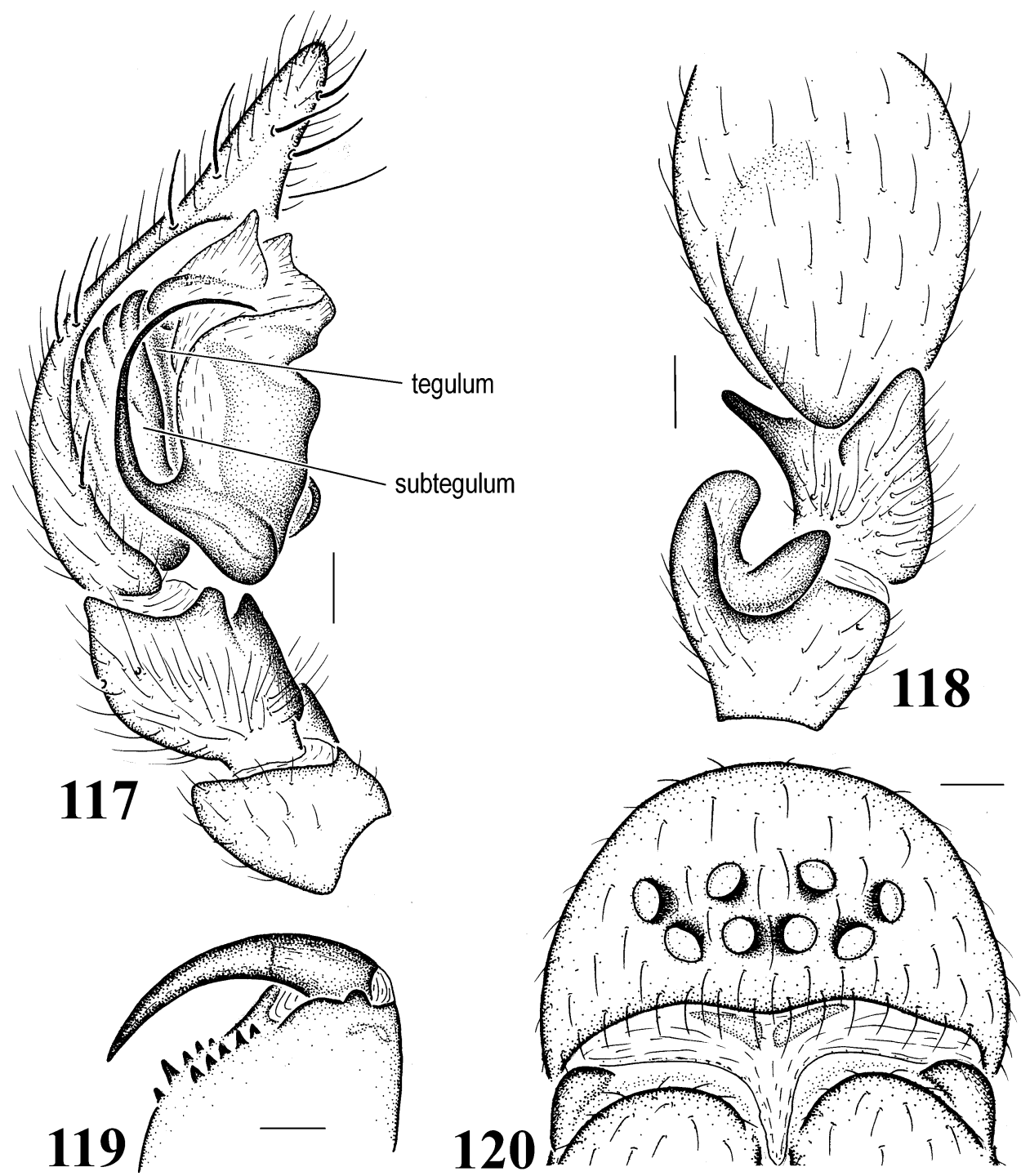

Figs. 117-120. Coelotes charitonovi Spassky. 117. Male palp, retrolateral view. 118. Male palp, dorsal view, showing patellar apophysis. 119. Chelicera, ventral view. 120. Carapace, front view.

relatively less broad patellar apophysis, a well-developed lateral tibial apophysis, a long conductor, and a slender conductor dorsal apophysis (129-131).

This species group is well represented in southern China.

There are at least two species in the pseudoterrestris group.

1. Coelotes pseudoterrestris Schenkel, 1963: female holotype from Yunnan, China, in MNHN and NHMB, examined.
The species Coelotes sacratus Wang et al., 1990, with male and female paratypes from Xishan, Kunming, Yunnan, China, in HBI, examined, is placed as a junior synonym of C. pserdoterrestris Schenkel (because of the identical female genitalic morphology). NEW SYNONYMY.

2. Coelotes yunnanensis Schenkel, 1963: female holotype from Yunnan, China, in MNHN, examined.

Lehtinen (1967) synonymized C. yunna- 

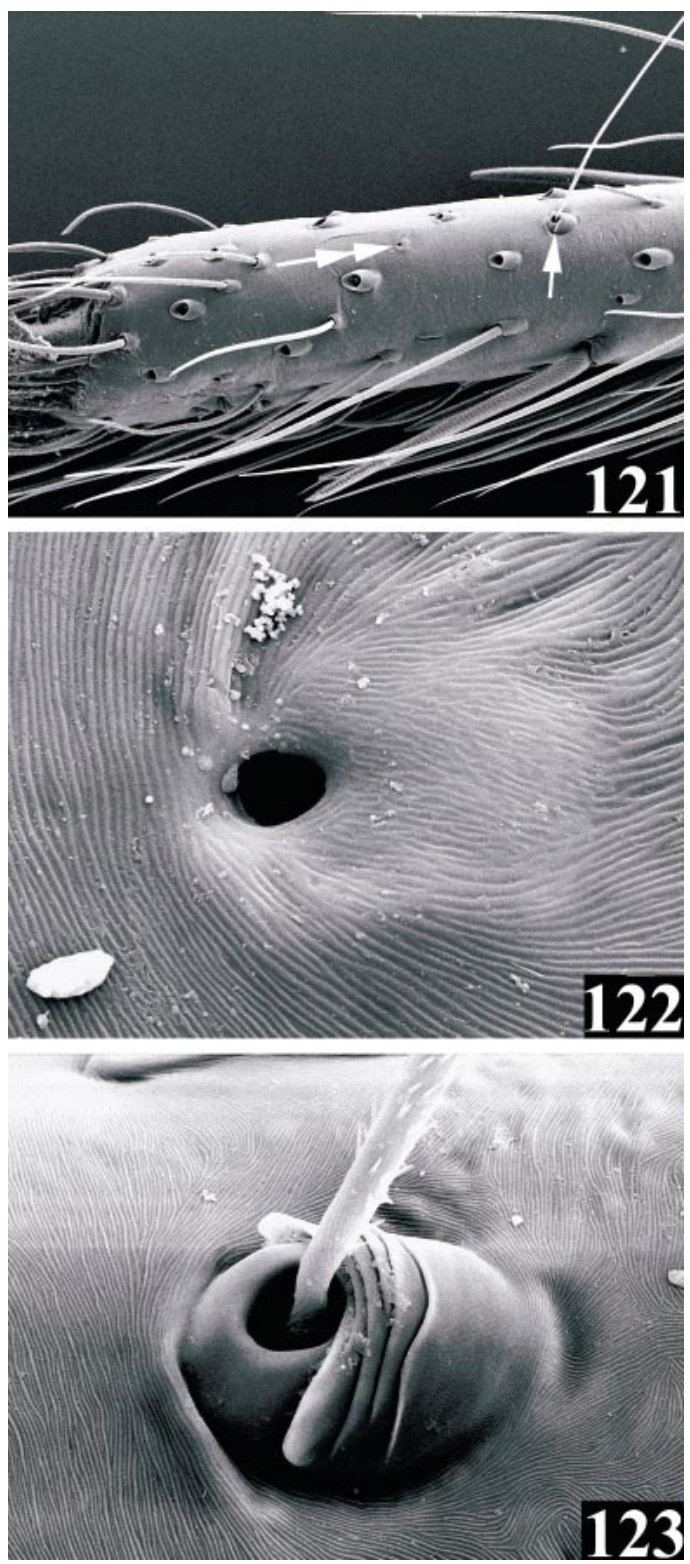

Figs. 121-123. Coelotes charitonovi Spassky, female. 121. Tarsus, showing position of tarsal organ (double arrows) and the distalmost trichobothrium (single arrow). 122. Tarsal organ. 123. Trichobothrium.

nensis with Paracoelotes spinivulvus (Simon, 1880). Examination of type specimen shows that this species is not a synonymy of Paracoelotes spinivulvus.
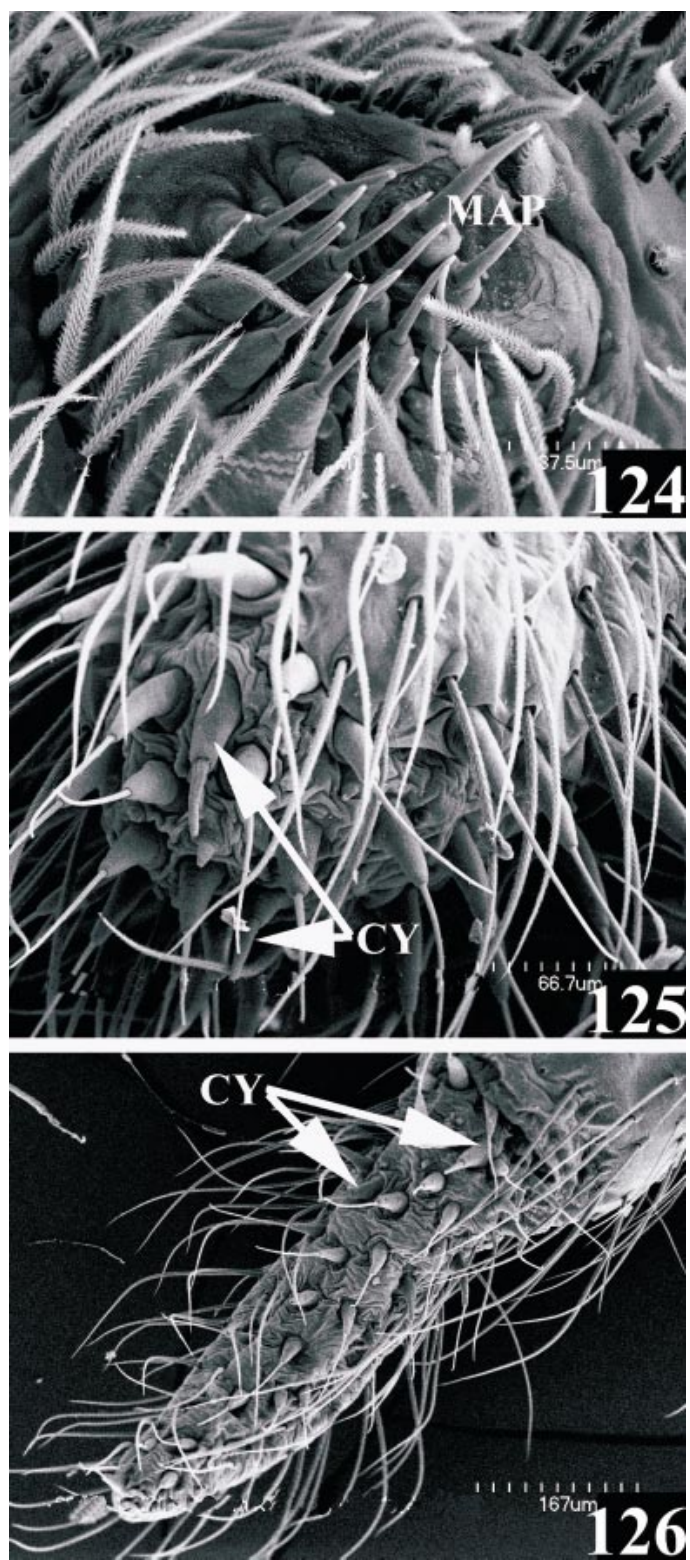

Figs. 124-126. Coelotes charitonovi Spassky, female spinnerets, ventral view, left. 124. ALS. 125. PMS. 126. PLS. Abbreviations are spelled out on p. 6.

Coelotes pseudoterrestris Schenkel Figures 127-136

Coelotes pseudoterrestris Schenkel, 1963: 286, fig. 161 (female holotype from Lo Thoei Tong, China, in MNHN, examined). 


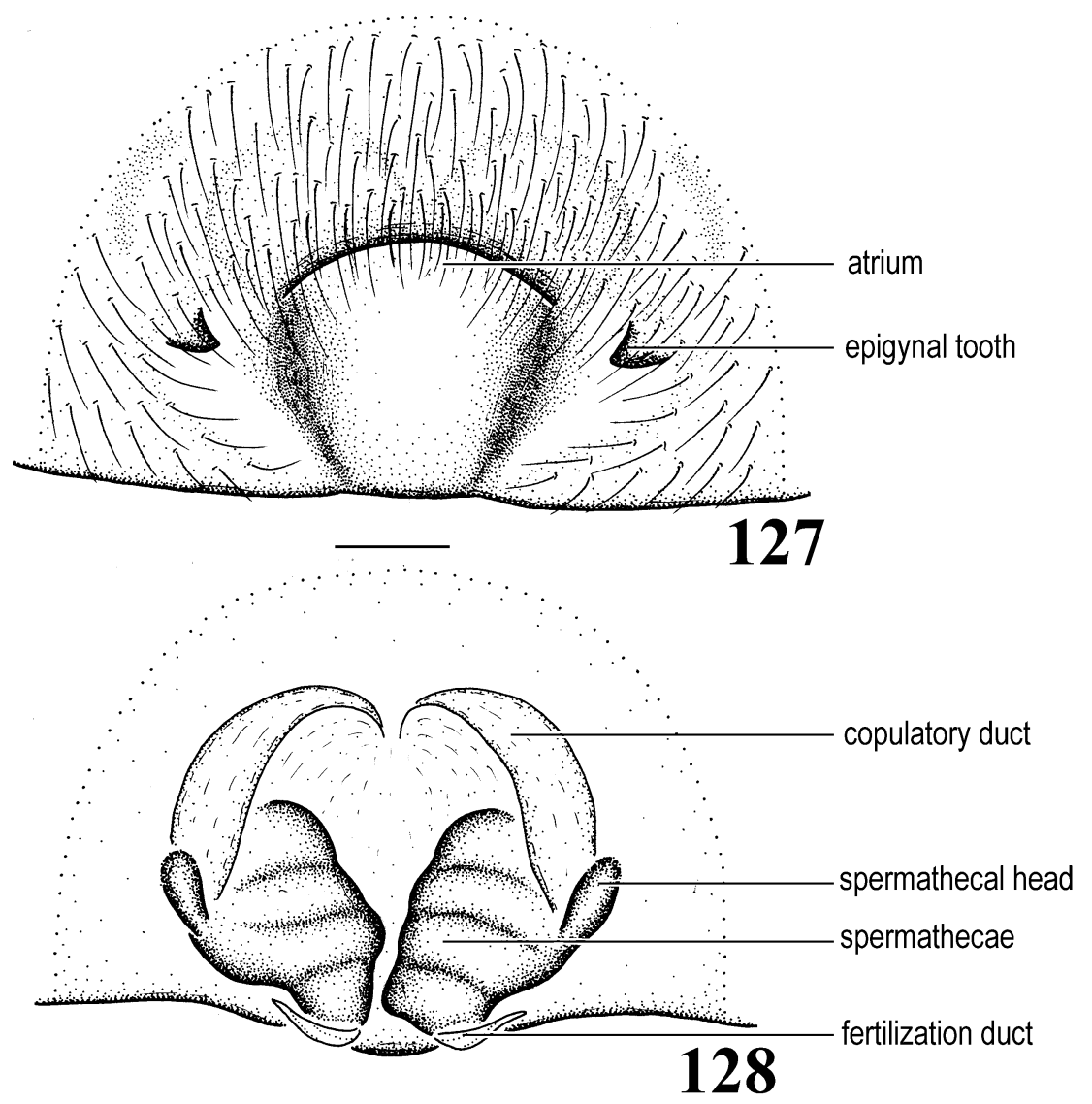

Figs. 127, 128. Coelotes pseudoterrestris Schenkel, female. 127. Epigynum. 128. Vulva.

Coelotes sacratus Wang et al., 1990: 222, figs. 103-107 (male and female types from Xishan, Kunming, Yunnan, China, in HBI, examined). - Song, et al., 1999: 378, figs. 224N, 224O, 226T, 228B.

Diagnosis: Females resemble C. yunnanensis but can be distinguished by the small epigynal teeth, the different shape of copulatory ducts, and the short, laterally situated spermathecal heads (figs. 127, 128). Males can be recognized by the shape of patellar apophysis, the long, slightly curved conductor, and the long, slender conductor dorsal apophysis (figs. 129-131).

DESCRIPTION: See also Schenkel (1963) and descriptions of $C$. sacratus by Wang et al. (1990). Total length 9.00-18.0. From front, anterior eye row slightly procurved, posterior row procurved; eye sizes and arrangements: AME slightly smaller than
ALE, PLE and PME subequal, slightly smaller than AME; AME-AME and AMEALE subequal, about two-thirds AME diameter, ALE-PLE close together, PLE-PME roughly 1.5 times their diameter, PME-PME slightly shorter than PME diameter. Clypeal height roughly twice AME diameter or slightly less; chilum divided, elongated (fig. 132). Chelicerae with three promarginal and three or four retromarginal teeth (fig. 133). Labium longer than wide. Length of female 1 st leg patella + tibia shorter than carapace length.

Female spinnerets: apex of ALS with 2 major ampullate gland spigots (MAP), 30 piriform gland spigots; PMS with 22 aciniform gland spigots, 2 cylindrical gland spigots (CY), 1 or 2 minor ampullate gland spigots (mAP); PLE with about 23 aciniform 


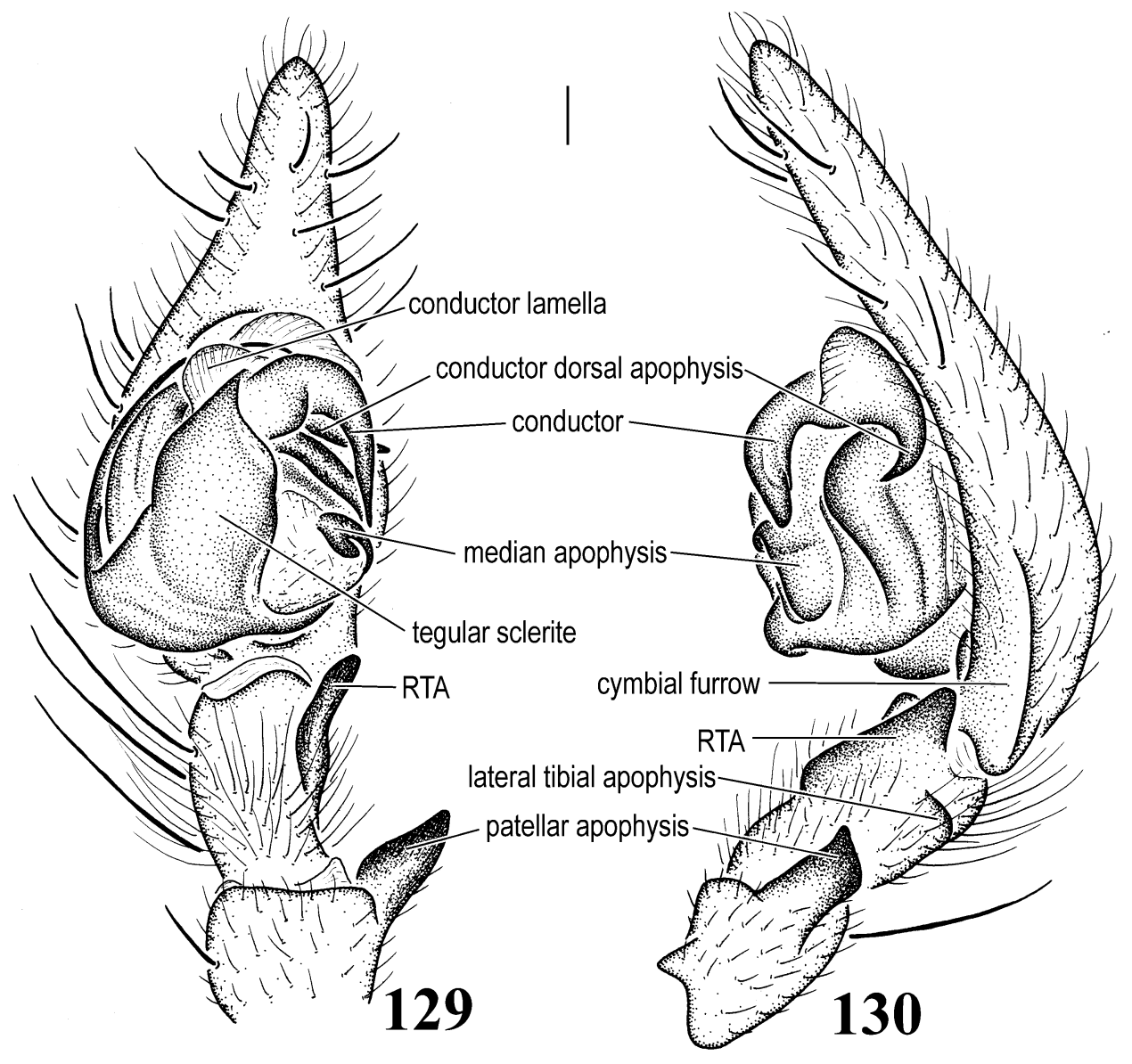

Figs. 129, 130. Coelotes pseudoterrestris Schenkel, male palp. 129. Ventral view. 130. Retrolateral view.

gland spigots and 2 cylindrical gland spigots (figs. 134-136).

Epigynum with epigynal teeth short, laterally situated; atrium reduced, only with more or less distinct anterior atrial margin; copulatory ducts broad, slightly sclerotized, with distinct shape; spermathecal heads situated laterad of spermathecae; spermathecae broad, convoluted, with indistinct bases and stalks (figs. 127, 128). Male palpi patellar apophysis relatively large, with apex slightly narrowed and curved ventrally; RTA long, with the distal end strongly extended beyond tibia; lateral tibial apophysis short, broad; cymbial furrow short; conductor long, slightly curved posteriorly; conductor lamella moderately developed; conductor dorsal apophysis long, slender, with sharp apex; embolus prolateral in origin, moderately long; median apophysis spoonlike, rounded, with no sharp distal end (figs. 129-131).

Notes: The locality of this species, Lo Thoei-Tong (Schenkel, 1963), has long been confusing. Brignoli (1983) wondered if it was in China. Zhu (1983) did not include this species (also $C$. denisi) in his list of spiders of China. The types of this species and $C$. denisi can be confidently regarded to be distributed in Yunnan (China) because (1) later large collections of this species were taken from Yunnan, and (2) 

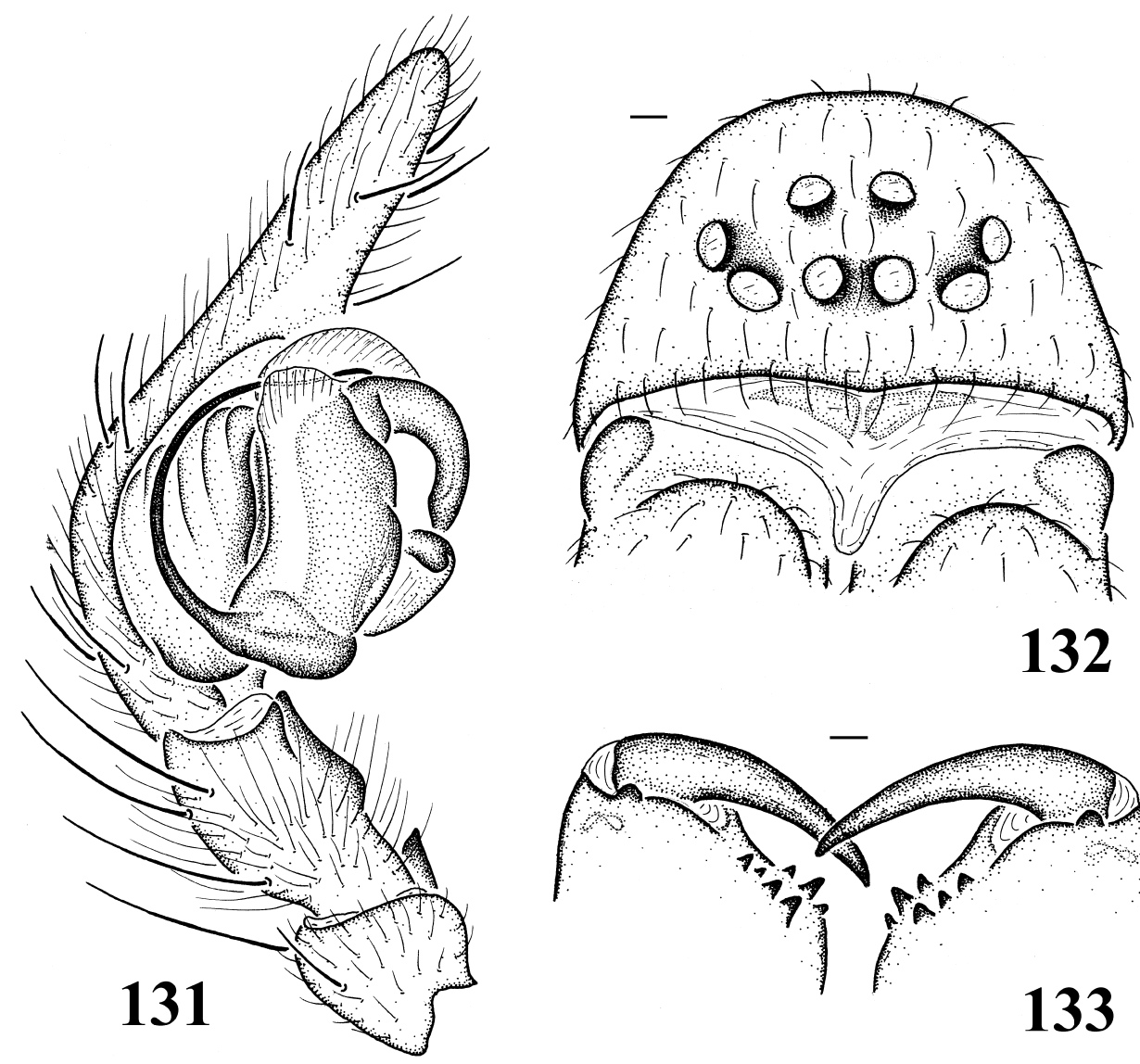

132

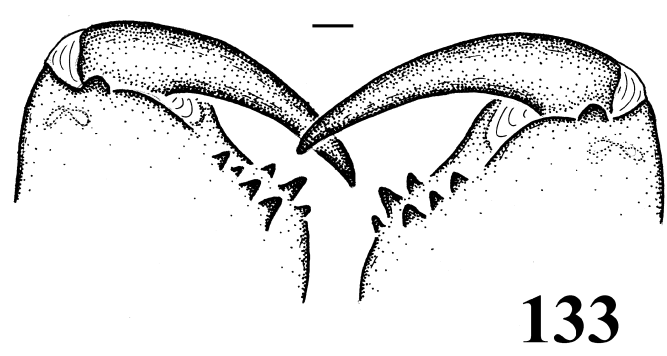

Figs. 131-133. Coelotes pseudoterrestris Schenkel. 131. Male palp, retrolateral view. 132. Carapace, front view. 133. Chelicerae, ventral view.

the types of $C$. yunnanensis, which has the close collection date (February 26, 1925) with $C$. pseudoterrestris and $C$. denisi (March 2, 1925), were collected from Yunnan fu, Yunnan.

Material Examined: CHINA: Yunnan: Lo Thoei-Tong, March 2, 1925, 1 female holotype type (MNHN, B2011 bis), 1 female co-type (NHMB, 1990a); Kunming, Xishan, October 24, 1986, 2 male and 2 female types of C. sacratus (J. F. Wang, HBI); Kunming, Xishan, Dragon Gate, 2000-2400 m, December 26, 1988, 1 male and 1 female (P. Beron, COLL. DEELEMAN-REINHOLD); Kunming, bamboo temple, December 29, 1988, 1 female (P. Beron, COLL. DEELEMAN-REINHOLD); Menzi County, v. Wulichong, January 6,
1989, 1 male and 1 female (P. Beron, COLL. DEELEMAN-REINHOLD); Menzi County, under stones near the village on the peathean, 1600-1800 m, January 31, 1989, 1 male (P. Beron, COLL. DEELEMAN-REINHOLD); Baoshan Prefecture, Pass over Gaoligongshan at $2100 \mathrm{~m}$ alt., Nankang, 36 air km SE TengChong, $24^{\circ} 50^{\prime} \mathrm{N}, 98^{\circ} 47^{\prime} \mathrm{E}$, native forest, November 4-7, 1998, 6 males (C. Griswold, D. Kavanaugh, C.L. Long, CAS); Baoshan Prefecture, pass over Gaoligongshan at 2300 $\mathrm{m}$, Luoshuidong, 28 air $\mathrm{km} \mathrm{E}$ TengChong, $24^{\circ} 57^{\prime} \mathrm{N}, 98^{\circ} 45^{\prime} \mathrm{E}$, native forest, October 26-31, 1998, 6 females and 8 males (C. Griswold, D. Kavanaugh, C.L. Long, CAS).

Distribution: China. 

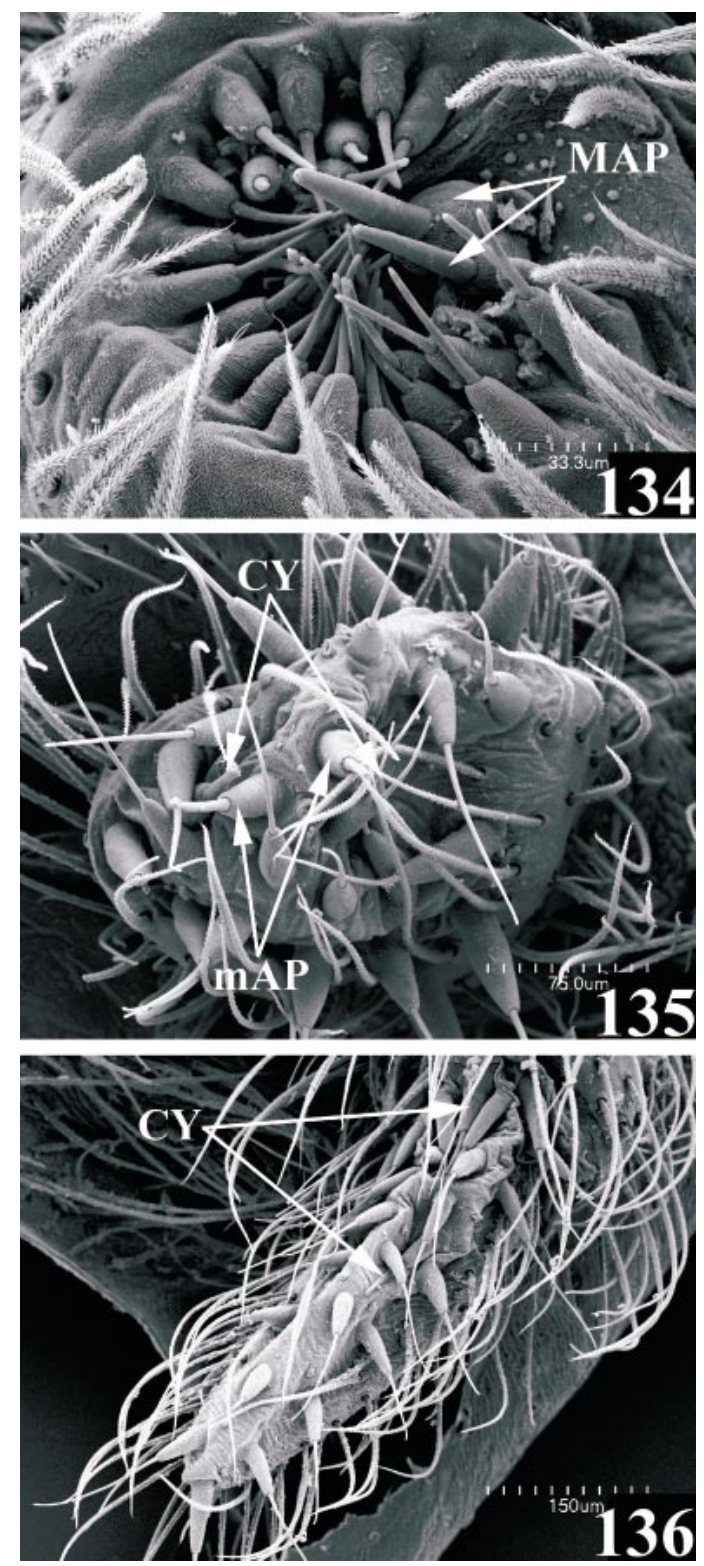

Figs. 134-136. Coelotes pseudoterrestris Schenkel, female spinnerets, ventral view. 134. ALS, left. 135. PMS, left. 136. PLS, right. Abbreviations are spelled out on p. 6.

\section{CORAS SIMON}

Coras Simon, 1898: 258. - Roewer, 1954: 58; Brignoli, 1983: 474; - Platnick, 1989: 424; Platnick, 1993: 574; - Platnick, 1997: 666.

TyPE SPECIES: Tegenaria medicinalis Hentz, 1821, by original designation.
DiAGNOSIS: Easily recognized by the large AME (larger than ALE) (fig. 147), combined with the anteriorly wide apart, posteriorly converging lateral atrial margins and the anterolaterally situated epigynal teeth of females (figs. 137-142) and by the presence of tegular carina in males (figs. 143-146).

DESCRIPTION: See description of type species (below).

Distribution: Eastern North America, from Nova Scotia to Florida (map 5).

Composition: Seventeen species are currently included. Some species show strong intraspecific variations in the genitalia of both sexes. A number of synonyms may exist.

The Chinese species C. rugosus Wang, Peng and Kim, 1996 and C. globasus Wang, Peng and Kim, 1996 are apparently not Coras species, and are only temporarily listed here.

1. Coras aerialis Muma, 1946: male holotype and female paratype from Maryland, in $\mathrm{AMNH}$, examined.

2. Coras alabama Muma, 1946: male holotype and female paratype from Alabama, in AMNH, examined.

3. Coras angularis Muma, 1944: male holotype and female paratype from Maryland, in $\mathrm{AMNH}$, examined.

4. Coras cavernorum Barrows, 1940: female holotype from Waynesville, North Carolina, in OSU, examined.

5. Coras crescentis Muma 1944: female holotype from Dans Rock, Maryland, in AMNH, examined.

6. Coras furcatus Muma, 1946: male holotype and female paratype from Pensacola, Florida, in AMNH, examined.

7. Coras globasus Wang, Peng and Kim, 1996: female holotype from Zhangjiajie, Hunan, China, in HBI, examined.

8. Coras juvenilis (Keyserling, 1881): female juvenile types from Haunted Cave, Kentucky, in MCZ, examined. Males and females from eastern North America examined.

9. Coras kisatchie Muma, 1946: male holotype and female paratype from Louisiana, in AMNH, examined.

10. Coras lamellosus (Keyserling, 1887): types (not designated) not examined. Males 

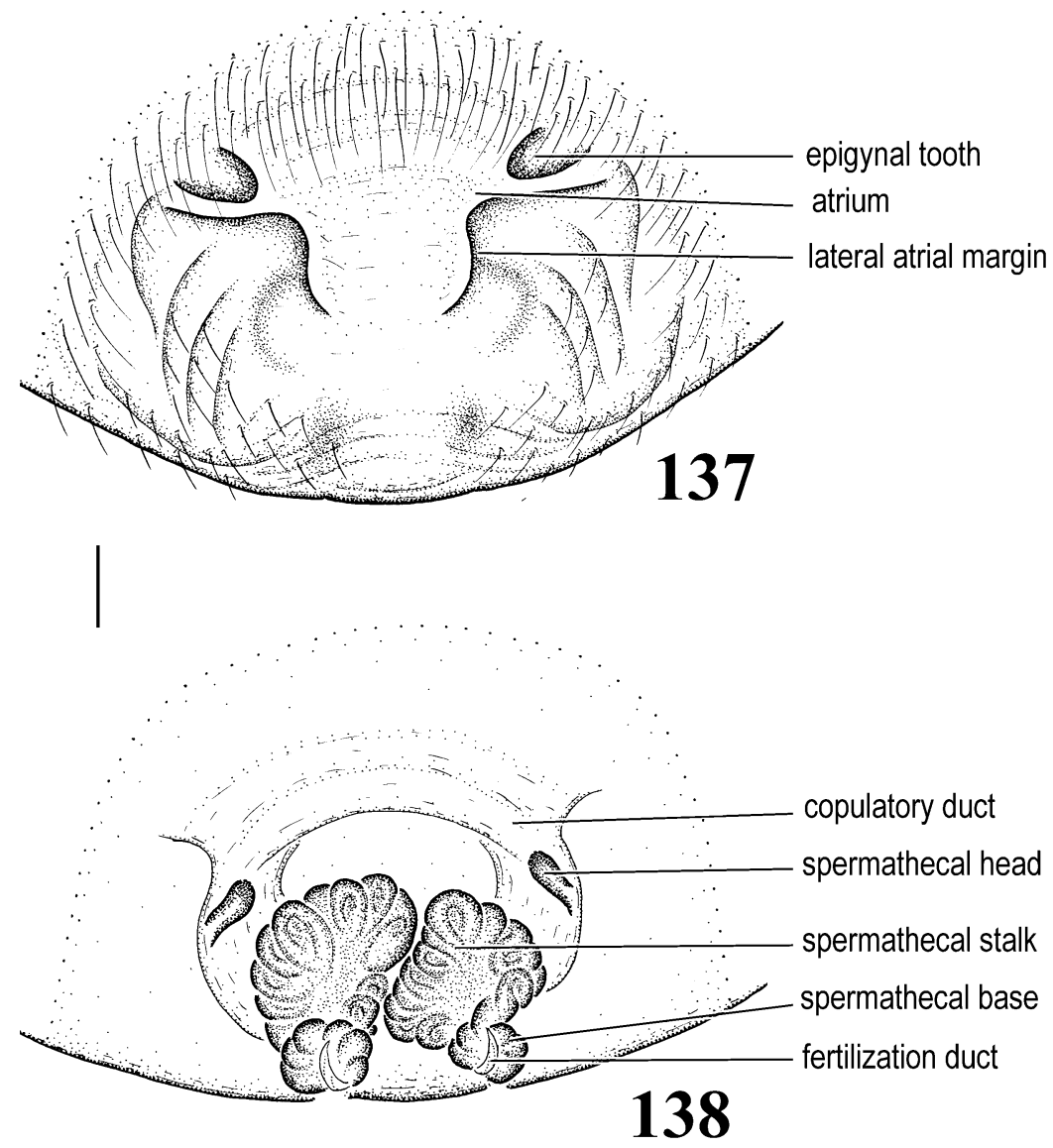

Figs. 137, 138. Coras medicinalis (Hentz), female. 137. Epigynum. 138. Vulva.

and females from eastern North America, in $\mathrm{AMNH}$, examined.

11. Coras medicinalis (Hentz, 1821): types (not designated) not examined. Males and females from eastern North America, in $\mathrm{AMNH}$, examined.

12. Coras montanus (Emerton, 1890): types (not designated) not examined. Males and females from eastern North America, in AMNH, examined.

13. Coras parallelis Muma, 1944: female holotype from Princess Anne, Maryland, in AMNH, examined.

14. Coras perplexus Muma, 1946: male holotype, male and female paratype from Mississippi, in AMNH, examined.

15. Coras rugosus Wang, Peng and Kim, 1996: female holotype from Chenbu, Hunan, China, in HBI, examined.

16. Coras taugynus Chamberlin, 1925: fe- male holotype and paratype from North Caronila (in MCZ), male and female paratypes from Alabama, in AMNH examined.

17. Coras tennesseensis Muma, 1946: male holotype and female paratype from Tennessee, in AMNH, examined.

\section{Coras medicinalis (Hentz)}

Figures 137-157

Tegenaria medicinalis Hentz, 1821: 53, T. 5, fig. 1. - Hentz, 1847: 462, T. 24, fig. 21; - Hentz, 1867: 107, fig. 110; - Emerton, 1902: 99, figs. 233-240, 244.

Clubiona medicinalis: Walckenaer, 1837: 607.

Tegenaria nemorensis Walckenaer, 1842: 10.

Coelotes urbanus Keyserling, 1887: 467, T. 6, fig. 31.

Coras medicinalis: Simon, 1898: 258, fig. 250; Comstock, 1912: 592, fig. 672; - Chamberlin, 1925: 122; - Comstock, 1940: 610, fig. 672; - 

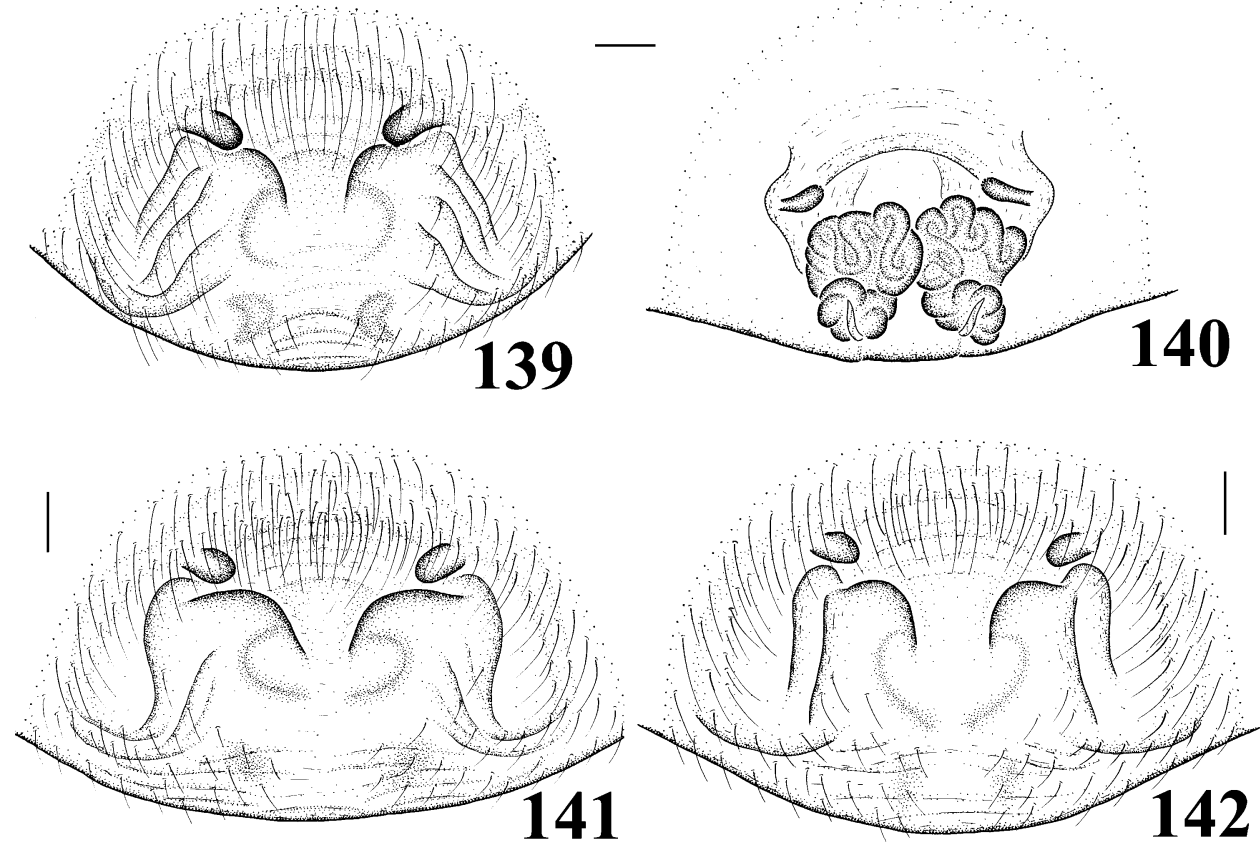

Figs. 139-142. Coras medicinalis (Hentz), female, showing variation. 139. Epigynum. 140. Vulva. 141. Epigynum. 142. Epigynum.

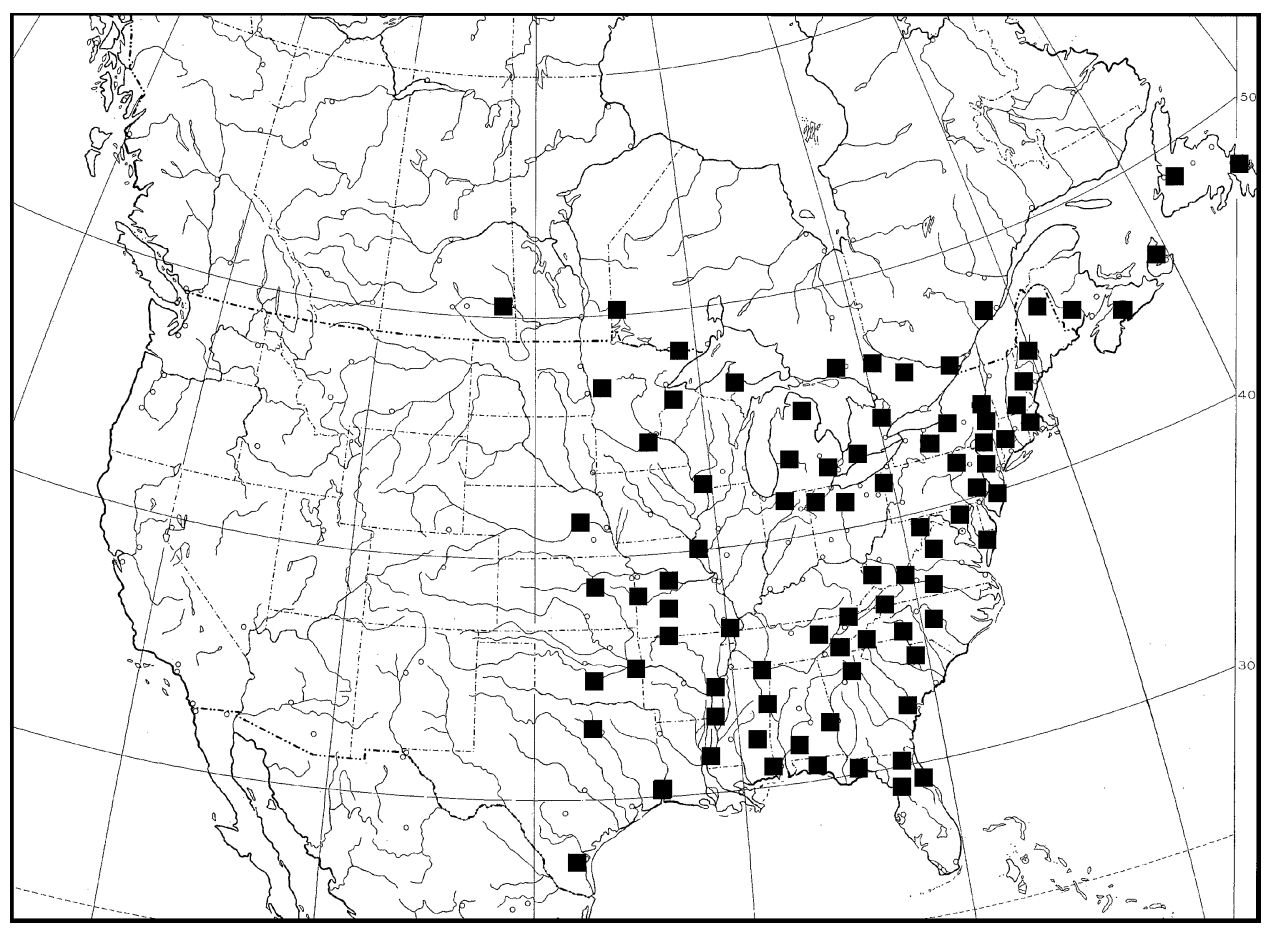

Map 5. Records of Coras. 


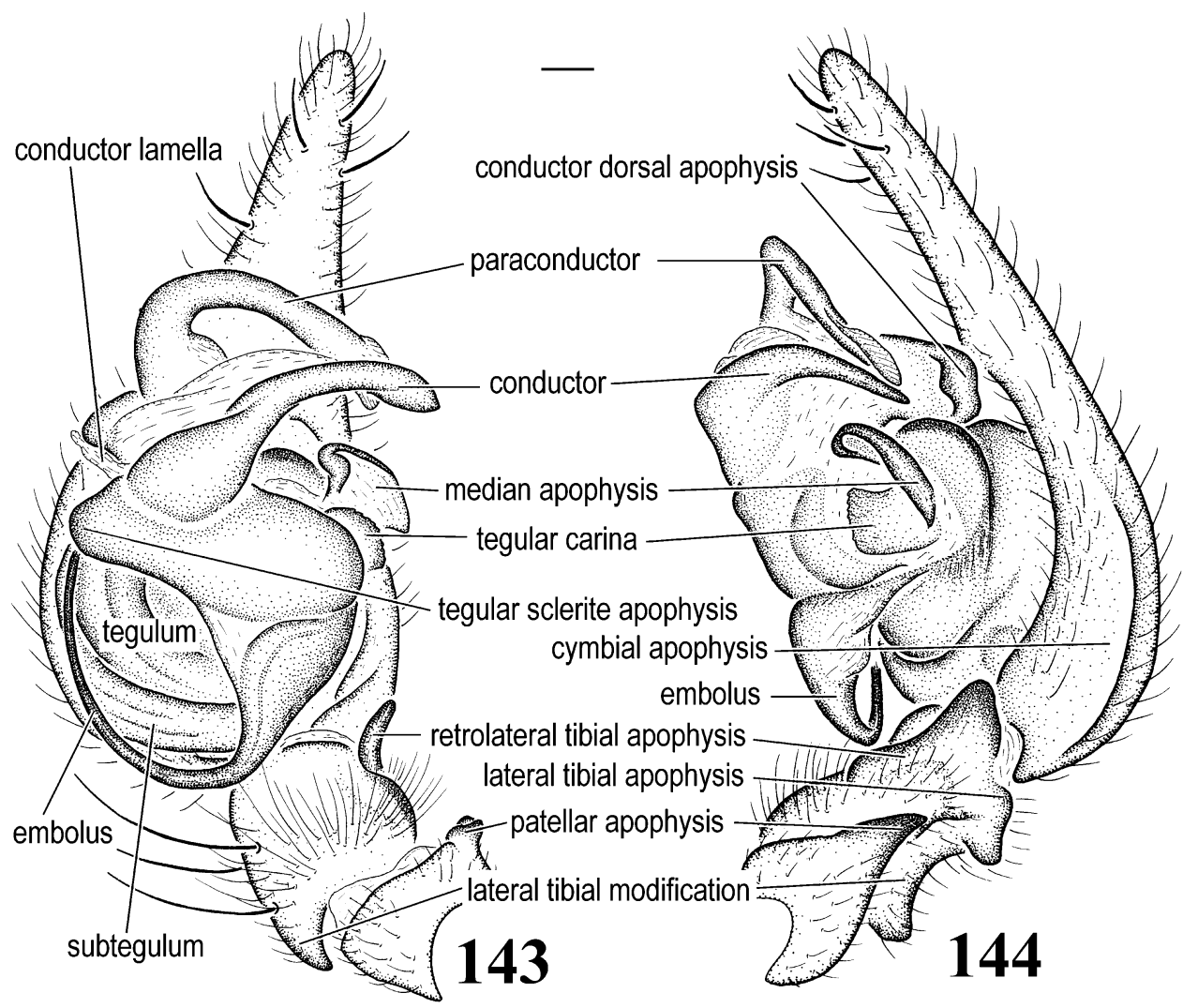

Figs. 143, 144. Coras medicinalis (Hentz), male palp. 143. Ventral view. 144. Retrolateral view, showing conductor dorsal apophysis.

Muma, 1943: 41, pl. XII, fig. 1; - Muma, 1946: 4, figs. 1-3, 21-24; - Kaston, 1948: 28, figs. 900-902, 914, 915; - Gering, 1953: 37, fig. 61 - Roth, 1982: 7, 8, figs. 35, 36; - Roth, 1985: B1-7, figs. 35, 36; - Roth, 1994: 53, figs. 35, 36.

Coras montanus: Roth and Brame, 1972: 27, fig. 35 (female, misidentified).

Coras juvenilis: Roth and Brame, 1972: 27, fig. 38 (male, misidentified).

Diagnosis: Females of this species can be distinguished from $C$. furcatus only by the presence of semicircular markings on the epigynal plate and from $C$. parallelis by the position of the lateral atrial margins (figs. 137, 139, 141). Males can be recognized by the short, nonbifid patellar apophysis (fig. 144).

DESCRIPTION: Total length about 6.0013.0, with considerable variation among individuals of the same species (Muma, 1946). From front, anterior eye row more or less straight, posterior row procurved; eye sizes and arrangements: AME largest, ALE subequal or slightly larger than PLE, PME smallest; AME may be separated by roughly their radius, AME-ALE close together and subequal to ALE-PLE, PME-PLE about 1.20-1.50 PME diameter, PME-PME slightly shorter than PME-PLE. Clypeal height about AME diameter, covered with long, strong setae; chilum divided, hairless, long (fig. 147). Chelicerae with three promarginal and three retromarginal teeth (fig. 148). Labium subequal in width and length or slightly wider, slightly notched distally.

Tarsal organ situated close to distal end of tarsus, slightly anterior of most distal trichobothrium (figs. 149-151). Apex of ALS with 2 major ampullate gland spigots (MAP), 2540 piriform gland spigots in both sexes; PMS with 1 or 2 minor ampullate gland spigots (mAP) and 40-45 aciniform gland spigots 




147

Figs. 145-148. Coras medicinalis (Hentz). 145. Male palp, prolateral view. 146. Male palp, retrolateral view. 147. Carapace, front view. 148. Chelicera, ventral view.

(AC) in both sexes, 2 cylindrical gland spigots (CY) in female; PLS with about 65 aciniform gland spigots in both sexes and 2 cylindrical gland spigots in female (figs. 152157).

Epigynum with epigynal teeth short; lateral atrial margins wider apart anteriorly, convergent posteriorly; copulatory ducts short; spermathecal heads slightly elongated; spermathecal stalks large, strongly convoluted; spermathecal bases relatively small, strongly convoluted (figs. 137-142). Male palp with one patellar apophysis; retrolateral tibial surface strongly modified with lobes and concavity; RTA long, occupying most of tibia, with distal end slightly extended be- 



Figs. 149-151. Coras medicinalis (Hentz), female. 149. Tarsus, showing position of tarsal organ (double arrows) and the distalmost trichobothrium (single arrow). 150. Trichobothrium. 151. Tarsal organ.

yond tibia; lateral tibial apophysis small; prolateral tibia with three strong setae; cymbial furrow moderately long, with distinct dorsal edge; tegular sclerite with prolateral apophysis; conductor long, slender, with paraconductor between conductor and conductor


Figs. 152-154. Coras medicinalis (Hentz), female spinnerets, ventral view, left. 152. ALS. 153. PMS. 154. PLS. Abbreviations are spelled out on p. 6.

dorsal apophysis; conductor dorsal apophysis strong; conductor lamella moderately developed; embolus basal in origin, moderately long, linear; median apophysis spoonlike, with extended distal end but not sharply pointed; tegular carina (the apophysis on te- 



Figs. 155-157. Coras medicinalis (Hentz), male spinnerets, ventral view, right. 155. ALS. 156. PMS. 157. PLS. Abbreviations are spelled out on p. 6.

gulum between median apophysis and tegular sclerite) situated parallel relative to tegular sclerite and median apophysis (figs. 143-146).

MATERIAL EXAMINED: Numerous males and females from eastern North America (for details, see generic revision by Platnick and Wang, in prep.).

DisTRIBUTION: East coast of United States from Connecticut, Rhode Island, New York, New Jersey, Pennsylvania, Maryland, Washington, D.C., Virginia, Delaware, North Carolina, South Carolina, Georgia, Alabama, to Florida.

\section{CORONILLA WANG}

Coronilla Wang, 1994: 281. - Platnick, 1997: 667.

TYPE SPECIES: Coronilla gemata Wang, 1994, by original designation. Female holotype and male allotype from Mt. Zhangjiajie, Dayong, Hunan, China, in HTU, examined.

Diagnosis: Females of Coronilla can be easily distinguished by the absence of epigynal teeth, the presence of a broad, transverse atrial septum, and the posteriorly expanded epigynal posterior margin (figs. 158, 159), males by the presence of two patellar apophyses and a reduced lateral tibial apophysis (figs. 161-168).

DESCRIPTION: See description of type species (below).

Distribution: China, Vietnam (map 6).

Composition: Two species are included.

1. Coronilla gemata Wang, 1994: female holotype, male and female paratypes from Mt. Zhangjiajie, Dayong, Hunan, China, in HTU, examined.

2. Coronilla sigillata Wang, 1994: female holotype, male and female paratypes from Mt. Tianmushan, Zhejiang, China, in HTU, examined.

\section{Coronilla gemata Wang \\ Figures 158-180}

Coronilla gemata Wang, 1994: 281, figs. 1-5 (female holotype, male and female paratypes from Mt. Zhangjiajie, Dayong, Hunan, China, in HTU, examined). - Song et al., 1999: 389, figs. 229G, 229H, 229K-M.

Coelotes yoshikoae Nishikawa, 1995: 141, figs. 1-8 (types from Vinh Phu Prov., Vietnam, in National Science Museum (Tokyo), not examined). NEW SYNONYMY.

Coelotes huangsangensis Peng et al., 1998: 77, figs. 1-6 (female holotype and male allotype from Huangsang, Suining, Hunan, China, in HBI, examined). NEW SYNONYMY.

Diagnosis: Females can be distinguished 

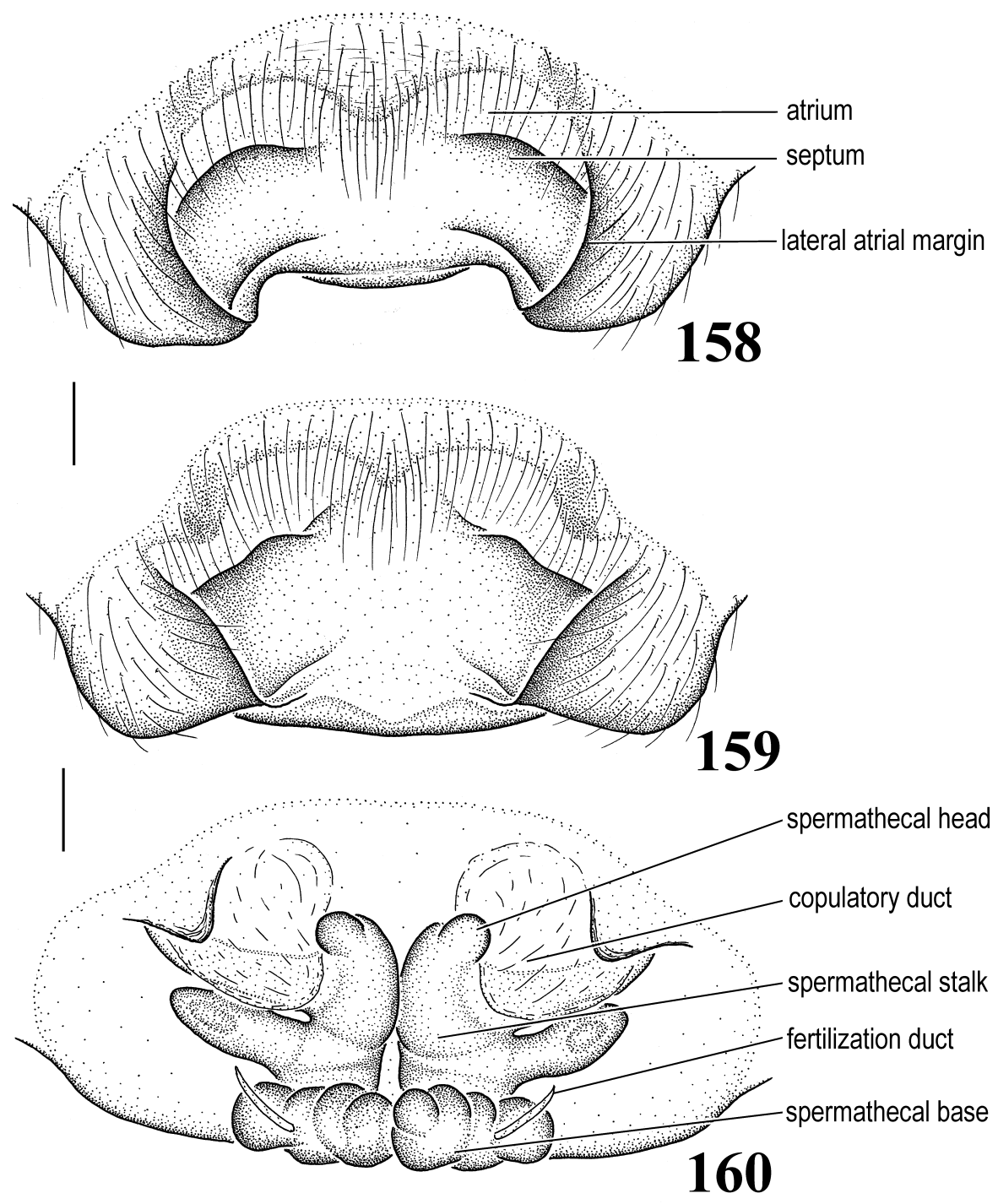

Figs. 158-160. Coronilla gemata Wang, female. 158. Epigynum. 159. Epigynum, view from different angle. 160. Vulva.

from $C$. sigillata by the different spermathecal shape (fig. 160), males by the relatively small conductor, the broad conductor ventral apophysis, and the short conductor dorsal apophysis (figs. 161-163).

DESCRIPTION: Total length 5.00-13.0, with considerable intraspecific variation. From front, anterior eye row slightly procurved, posterior row procurved; AME slightly smaller than ALE or subequal, PLE subequal to PME, slightly smaller than AME and ALE; AME separated by about their radius and slightly longer than AME-ALE, ALEPLE close together, PME separated by about their diameter, separated from PLE by about 1.25-2.00 times their diameter; clypeal height about 1.50 times AME diameter, covered with long, strong setae; chilum divided, hairless, long (fig. 169). Chelicerae with three promarginal and four retromarginal teeth (fig. 170). Labium longer than wide. Length of 1st leg patella + tibia shorter than carapace length.

Trachea examined, simple (fig. 171). Tar- 


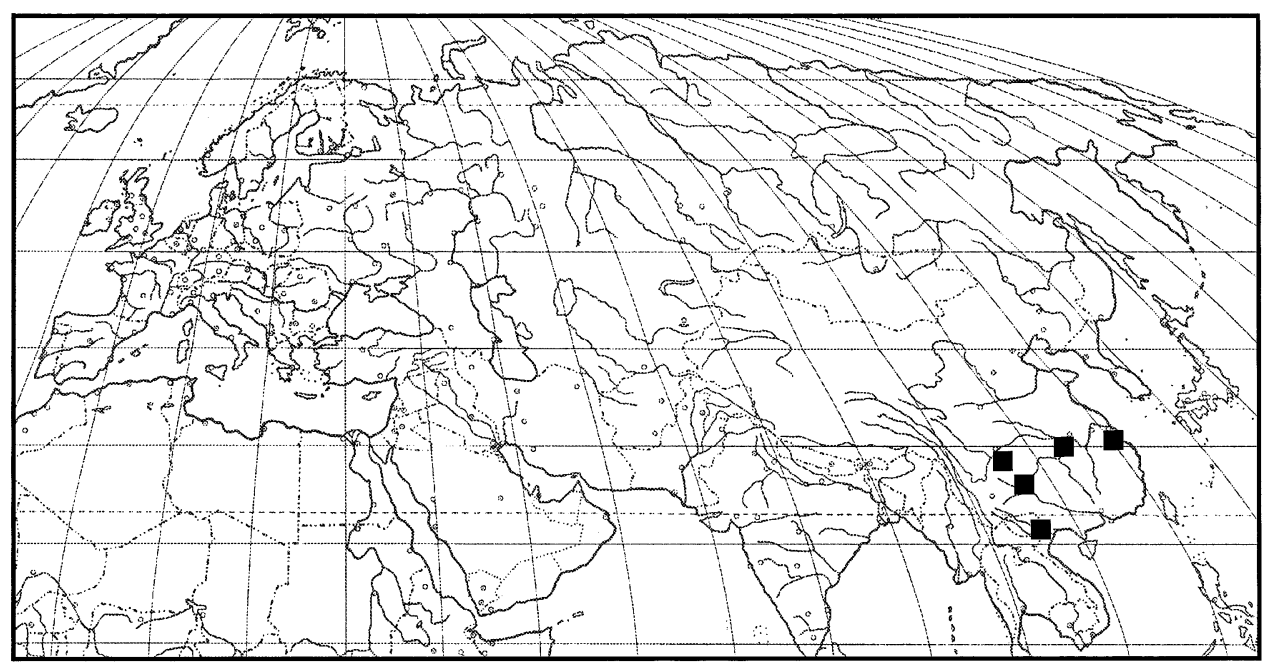

Map 6. Records of Coronilla.

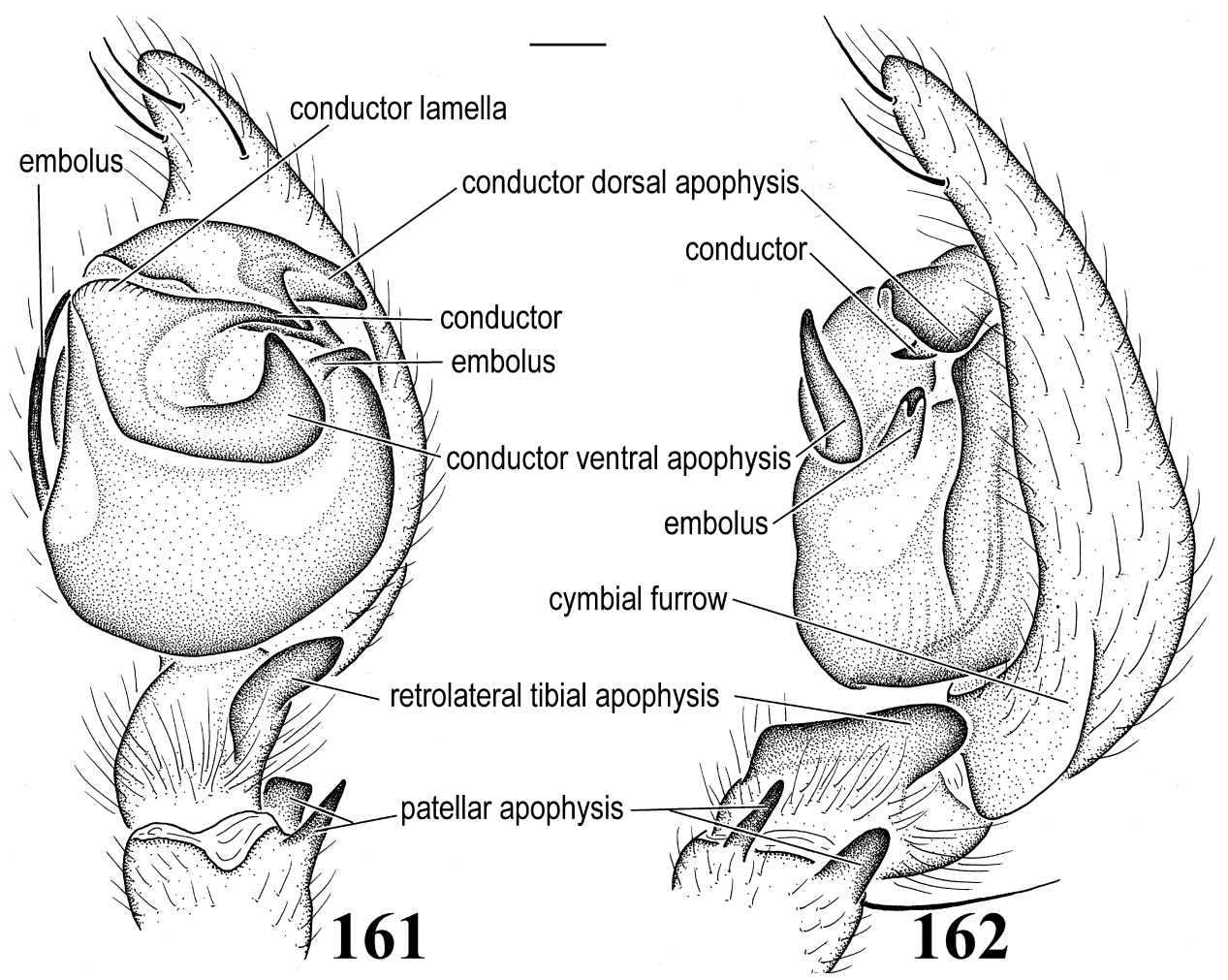

Figs. 161, 162. Coronilla gemata Wang, male palp. 161. Ventral view. 162. Retrolateral view. 


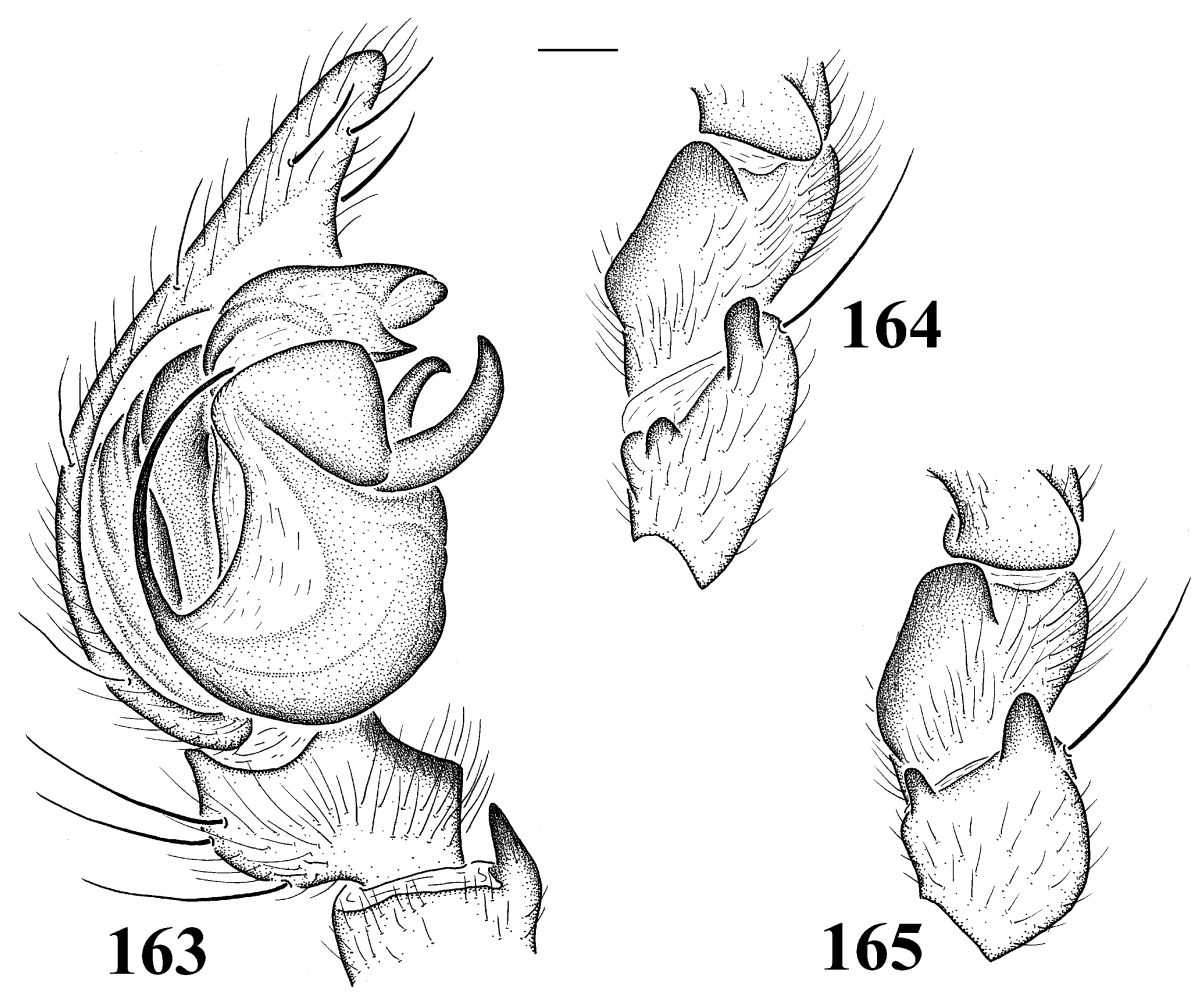

Figs. 163-165. Coronilla gemata Wang, male palp. 163. Prolateral view. 164. Retrolateral view of tibia and patellar, showing variation of patellar apophyses length. 165. Tibia and patellar, showing variation of patellar apophyses length.

sal organ situated close to distal end of tarsus, slightly anterior of distalmost trichobothrium (figs. 172-174). Apex of ALS with 2 major ampullate gland spigots (MAP), 6075 piriform gland spigots in both sexes; PMS with 1 or 2 minor ampullate gland spigots (mAP) and 13-16 aciniform gland spigots in both sexes, 2 cylindrical gland spigots (CY) in female; PLS with about 30-35 aciniform gland spigots in both sexes, and 2 cylindrical gland spigots in female (figs. 175-180).

Epigynum with large atrium; atrial septum broad, transversely situated; copulatory ducts large; spermathecal heads large, situated anteriorly; spermathecal stalks short, with lateral apophyses; spermathecal bases relatively large, transversely situated, more or less convoluted (figs. 158-160). Male palpal patellar apophyses with ventral one slender and long (occasionally short), dorsal one short and strong; RTA slightly shorter than tibia; lateral tibial apophysis absent; cymbial furrow mod- erately long; tegular sclerite apparent; conductor short; conductor ventral apophysis long, strong; conductor dorsal apophysis broad, relatively short; median apophysis slender, with distal end slightly curved or spiral (figs. 161-168).

SYNONYMS: The length of the male patellar ventral apophysis varies even in specimens from the same locality. The male of Coelotes huangsangensis has a relatively short ventral patellar apophysis. This situation also occurs in some males of $C$. gemata from the type locality. The female (allotype) genitalia of Coelotes huangsangensis, which is not fully sclerotized, is the same as C. gemata. As a result, Coelotes huangsangensis is considered to be a junior synonym of $C$. gemata. The species $C$. yoshikoae (types not examined), judged by the illustrations, is also considered to be a junior synonym of $C$. gemata.

Material Examined: CHINA: Hunan: 




Figs. 166, 167. Coronilla gemata Wang, male palp. 166. Expanded, prolateral view. 167. Expanded, ventral view.

Dayong, Mt. Zhangjiajie, November 5, 1985, female holotype, male allotype, 6 female and 7 male paratypes (J. F. Wang, HTU); Tianpingshan, October 16, 1986, 4 females and 4 males (J. F. Wang, HTU); Suining, Huangsang, October 14, 1996, female holotype and male allotype of $C$. huangsangensis Peng et al., 1998 (M. X. Liu, HBI). Sichuan: E-meishan, September 27, 1975, 2 females (C. D. Zhu, NBUMS, 75-2172); Chongqing, September 26, 1997, molted to adult later October, 1 male (X. P. Wang, AMNH).

DisTRIBUTION: Known from China (Hunan, Sichuan) and Vietnam (Vinh Phu).



Map 7. Records of Draconarius. 


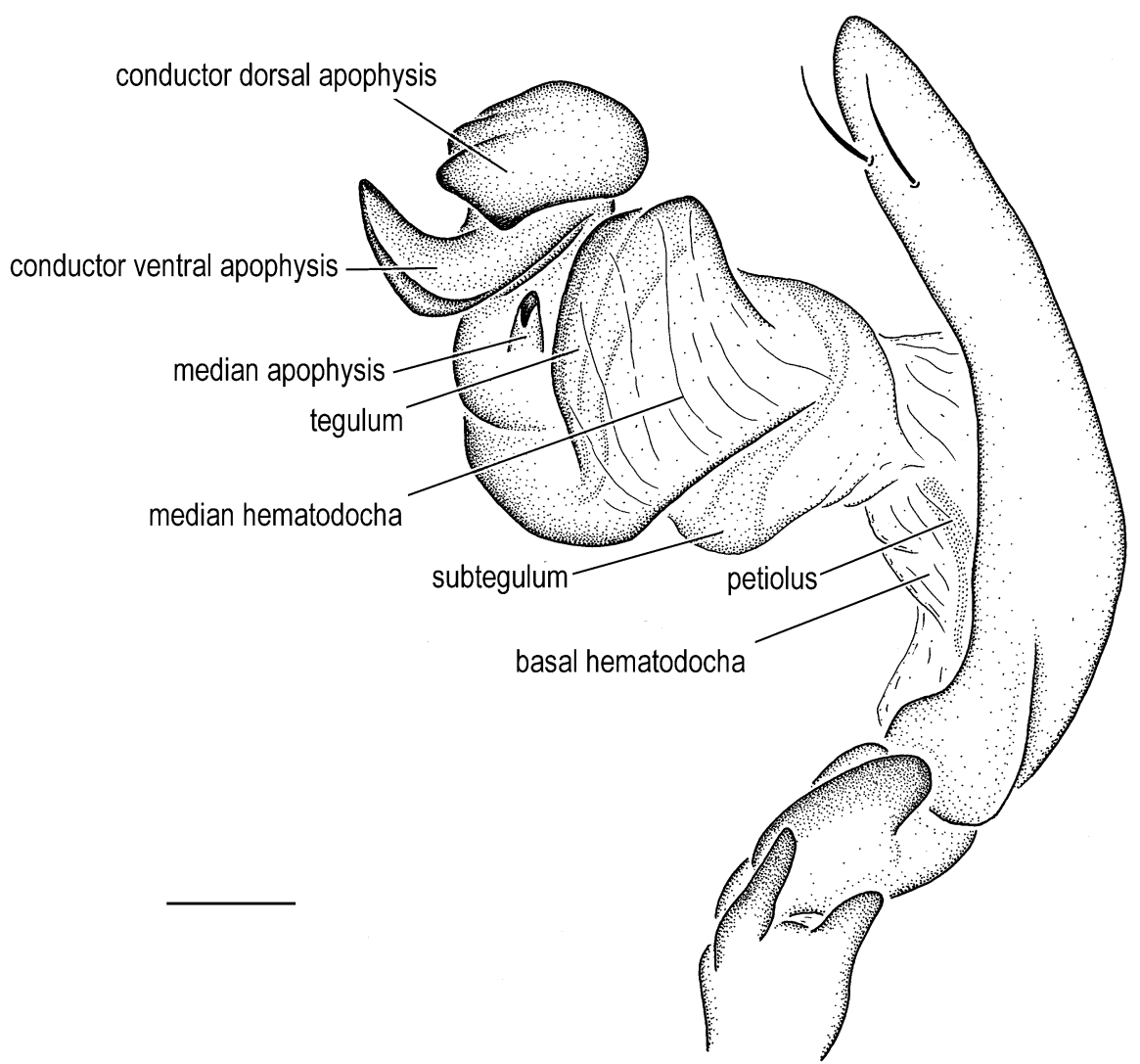

Fig. 168. Coronilla gemata Wang, male palp, expanded, retrolateral view.

\section{DRACONARIUS OVTCHINNIKOV}

Draconarius Ovtchinnikov, 1999: 70.

TyPe SPeCIEs: Draconarius venustus Ovtchinnikov, 1999, by original designation.

DiAgnosis: Resembles Asiacoelotes in having an elongated cymbial furrow, a long, slender embolus, and long, strongly convoluted spermathecae. Females can be distinguished by the posteriorly originated copulatory ducts and the widely separated spermathecae (figs. 182, 193); males by having a conductor dorsal apophysis (figs. 184, 185, 198).

DESCRIPTION: See description of Draconarius wudangensis (Chen and Zhao).

Distribution: Tadzhikistan, Bhutan, Nepal, China, Korea (map 7).

Composition: Twenty-six species are included. All are new combinations except the type species.

The species Draconarius wudangensis
(Chen and Zhao, 1997) and Draconarius sinualis (Chen, Zhao and Wang, 1991) are described here. The male of $D$. sinualis is described for the first time.

1. Draconarius arcuatus (Chen, 1984): female paratypes from Huanglongdong, Hangzhou, Zhejiang, China, in HTC, examined. NEW COMBINATION (transferred here from Coelotes).

2. Draconarius aspinatus (Wang et al., 1990): female holotype, male and female paratypes from Huangshan, Anhui, China, in HBI, examined. NEW COMBINATION (transferred here from Coelotes).

3. Draconarius baccatus (Wang, 1994): male and female paratypes from Xiangfan, Hubei, China, in HBI, examined. NEW COMBINATION (transferred here from Coelotes).

4. Draconariuss baronii (Brignoli, 1978): male holotype only, female paratype is a different species (of the genus Himalcoelotes). Male holotype from Dorjula, Bhutan, in 


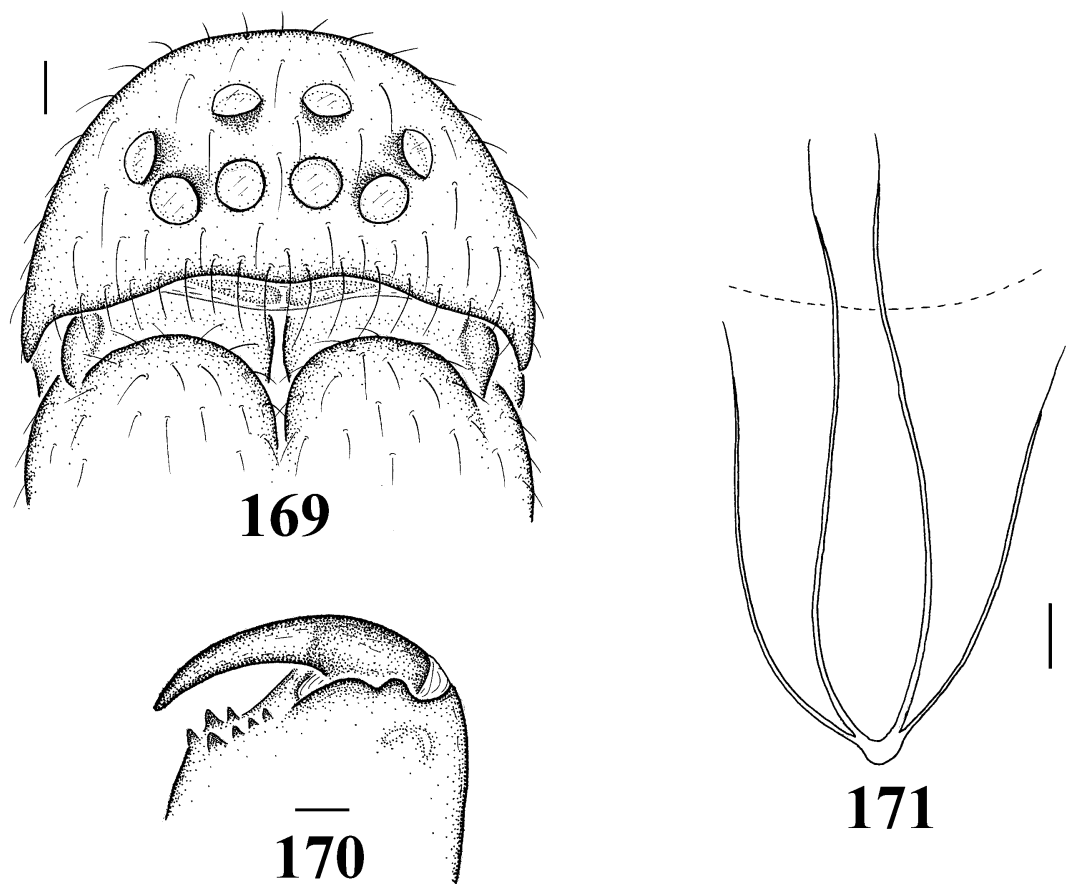

Figs. 169-171. Coronilla gemata Wang, female. 169. Carapace, front view. 170. Chelicera, ventral view. 171. Trachea, dashed line refers to position of epigastric furrow.

NHMB, examined. NEW COMBINATION (transferred here from Coelotes).

5. Draconarius calcariformis (Wang, 1994): female holotype and male paratype form Dabashan, Hubei, China, in HBI, examined. NEW COMBINATION (transferred here from Coelotes).

6. Draconarius cheni (Platnick, 1989): female holotype and paratype from Huanglongdong, Hangzhou, Zhejiang, China, in HTC, examined. NEW COMBINATION (transferred here from Coelotes).

7. Draconarius coreanus (Paik and Yaginuma, 1969): female holotype, male and female paratypes from Seong-gul Cave, Korea, in NSMT, not examined. NEW COMBINATION (transferred here from the genus Coelotes). Male and female specimens from Korea, in KAI, examined.

8. Draconarius davidi (Schenkel, 1963): female holotype from Inkiaphou, Shensi, China, in MNHN, examined. NEW COMBINATION (transferred here from Coelotes).

9. Draconarius gurkha (Brignoli, 1976): female holotype from Yak-Alm, Taboche,
Nepal, in ZII, examined. NEW COMBINATION (transferred here from Coelotes).

The species Coelotes lama Brignoli, 1976: female holotype from Nepal, in ZII, examined, has the identical genitalic morphology and is placed as a junior synonym of $D$. gurkha (Brignoli). NEW SYNONYMY (transferred here from Coelotes).

10. Draconarius infulatus (Wang et al., 1990): female holotype and 1 female paratype from Tianmushan, Zhejiang, China, in HBI, not examined. NEW COMBINATION (transferred here from Coelotes).

11. Draconarius labiatus (Wang and Ono, 1998): female holotype and paratype from Nantou, Taiwan, in NSMT, examined. NEW COMBINATION (transferred here from Coelotes).

12. Draconarius lutulentus (Wang et al., 1990): male and female paratypes from Zhangjiajie, Dayong, Hunan, China, in HBI, examined. NEW COMBINATION (transferred here from Coelotes).

13. Draconarius molluscus (Wang et al., 1990): female paratypes from Lushan, Ji- 

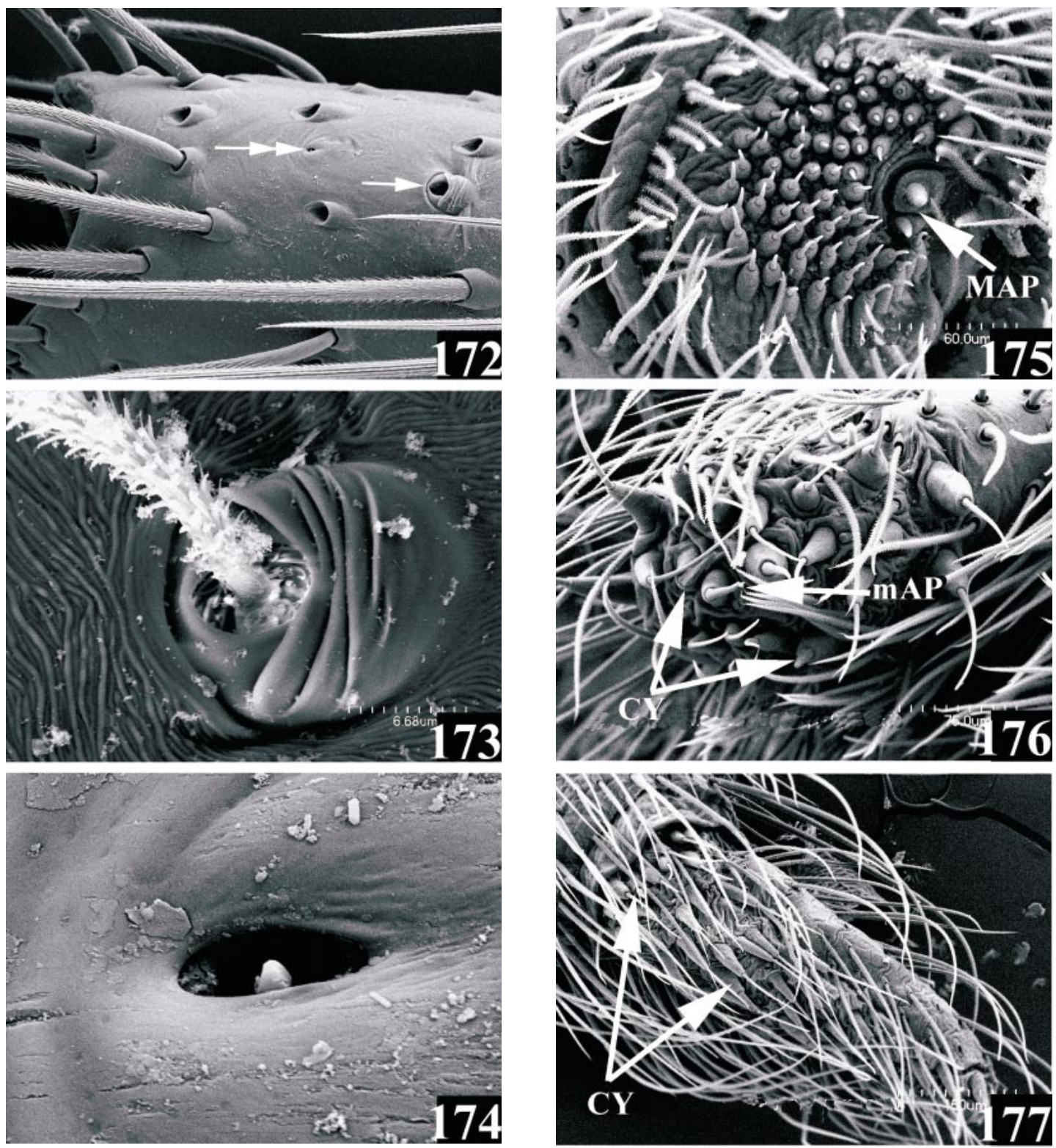

Figs. 172-174. Coronilla gemata Wang, female. 172. Tarsus, showing position of tarsal organ (double arrows) and the distalmost trichobothrium (single arrow). 173. Trichobothrium. 174. Tarsal organ.

angxi, China, in HBI, examined. NEW COMBINATION (transferred here from Coelotes).

14. Draconarius neixiangensis (Hu, Wang and Wang, 1991): female holotype and male and female paratypes from Neixiang, Henan, China, deposited in Shandong University, Ji-

Figs. 175-177. Coronilla gemata Wang, female spinnerets, ventral view. 175. ALS, left. 176. PMS, left. 177. PLS, left. Abbreviations are spelled out on p. 6 .

nan, China, not examined. NEW COMBINATION (transferred here from Coelotes).

15. Draconarius potanini (Schenkel, 1963): female holotype from Gansu, China, in MNHN, examined. NEW COMBINATION (transferred here from Cybanus). 



Figs. 178-180. Coronilla gemata Wang, male spinnerets, ventral view. 178. ALS, right. 179. PMS, right. 180. PLS, left. Abbreviations are spelled out on p. 6.

16. Draconarius schenkeli (Brignoli, 1978): female holotype from Chimakothi, Bhutan, in NHMB, examined. NEW COMBINATION (transferred here from Coelotes).

17. Draconarius singulatus (Wang et al., 1990): male and female paratypes from Nan- shanping, Chenbu, Hunan, China, in HBI, examined. NEW COMBINATION (transferred here from Coelotes).

18. Draconarius sinualis (Chen, Zhao and Wang, 1991): female holotype from Jinding, Wudangshan, Hubei, China, in HUW, examined. NEW COMBINATION (transferred here from Coelotes). Both male and female specimens from China (Hubei), in IZB, examined.

19. Draconarius stemmleri (Brignoli, 1978): female holotype and paratype from Sha Gogona, Bhutan, in NHMB and MCV, examined. NEW COMBINATION (transferred here from Coelotes).

20. Draconarius striolatus (Wang et al., 1990): female holotype from Yuzhong, Gansu, China, in HBI, examined. NEW COMBINATION (transferred here from Coelotes).

21. Draconarius trifasciatus (Wang and Zhu, 1991): female holotype and male paratype from Mt. Emei, Sichuan, China, in NBUMS, not examined. NEW COMBINATION (transferred here from Coelotes).

22. Draconarius venustus Ovtchinnikov, 1999: male holotype and female paratype from Yachsu River valley, Khazratisho Mt., Tadzhikistan, in CSO, not examined.

23. Draconarius wenzhouensis (Chen, 1984): female holotype and paratype from Xueshan, Wenzhou, Zhejiang, China, in HTC, examined. NEW COMBINATION (transferred here from Coelotes).

24. Draconarius wudangensis (Chen and Zhao, 1997): male and female paratypes from Jinding, Wudangshan, Hubei, China, in HUW, examined. NEW COMBINATION (transferred here from Coelotes).

25. Draconarius wuermlii (Brignoli, 1978): female holotype from Dechhi Paka, Bhutan, in NHMB, examined. NEW COMBINATION (transferred here from Paracoelotes).

26. Draconarius yosiianus (Nishikawa, 1999): female holotype and paratype from Jiabao Dong, Xingren Cun, Jiazhuan Xiang, Bama Xian, northwestern Guangxi, China, in NSMT, not examined. NEW COMBINATION (transferred here from Coelotes).

\section{Draconarius wudangensis (Chen and Zhao) Figures 181-191}

Coelotes wudangensis Chen and Zhao, 1997: 87, figs. 1-4 (male and female paratypes from Jind- 

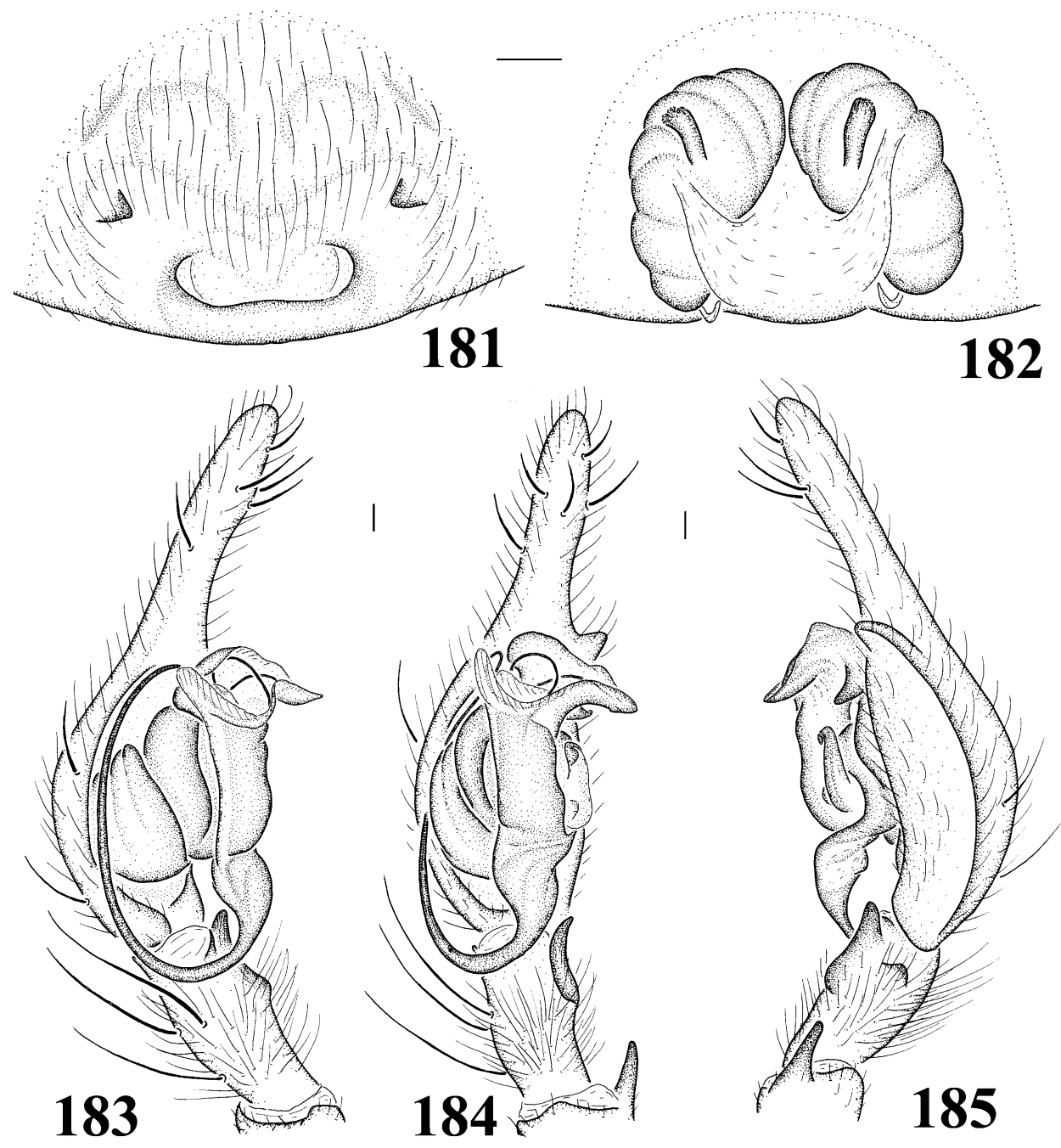

Figs. 181-185. Draconarius wudangensis (Chen and Zhao). 181. Female epigynum. 182. Female vulva. 183. Male palp, prolateral view. 184. Male palp, ventral view. 185. Male palp, retrolateral view.

ing, Wudangshan, Hubei, China, in HUW, examined). - Song et al., 1999: 388, figs. 226K, 226L, 227O, 229A.

Diagnosis: This species is similar to $D$. venustus but can be distinguished by the large, anteriorly situated spermathecal heads of females (fig. 182), the relatively short RTA, the less extended cymbial furrow, and the different shape of embolic base of males (figs. 183-185).

Description: Total length 6.00-12.0. From front, anterior eye row slightly procurved, posterior row procurved; eye sizes and arrangements: AME smallest, ALE largest,
PLE slightly larger than PME; AME-AME slightly shorter than their diameter, AMEALE separated by roughly AME radius, ALE-PLE close together, PME-PLE separated by more than their diameter, PME-PME slightly wider than their diameter. Clypeal height about twice AME diameter or slightly less; chilum divided, elongated. Chelicerae with three promarginal and two retromarginal teeth. Labium slightly shorter than wide. Length of female 1st leg patella + tibia longer than carapace length.

Tarsal organ situated dorsally, away from distal end of tarsus, close to second or third 



Figs. 186-188. Draconarius wudangensis (Chen and Zhao), female. 186. Tarsus, showing position of tarsal organ (double arrows) and the distalmost trichobothrium (single arrow). 187. Trichobothrium. 188. Tarsal organ.

distal trichobothrium (figs. 186-188). Female spinnerets examined: apex of ALS with 2 major ampullate gland spigots (MAP), about 46 piriform gland spigots; PMS not well prepared, with 1 minor ampullate gland spigots visible, at least 20 aciniform gland spigots, and 1 cylindrical gland spigot $(\mathrm{CY})$ observed; PLS with about 34 aciniform gland spigots and 2 cylindrical gland spigots (figs. 189-191).

Epigynum with epigynal teeth short, widely separated, situated anterior of atrium; atrium small; copulatory ducts originated posteriorly, mesal of spermathecae, more or less elongated; spermathecal heads apparent; spermathecae broad, elongated, convoluted, with indistinct stalks and bases (figs. 181, 182). Male palp with patellar apophysis long;
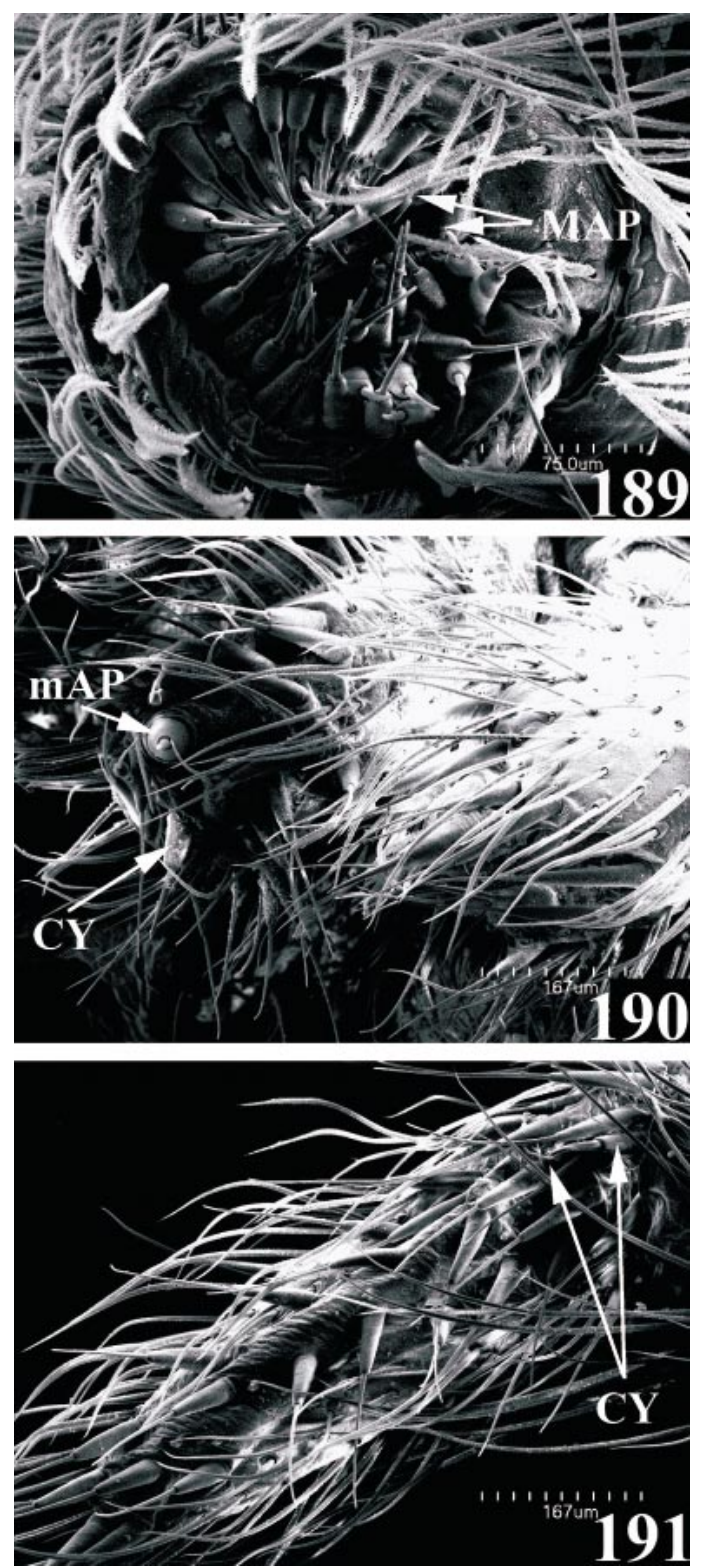

Figs. 189-191. Draconarius wudangensis (Chen and Zhao), female spinnerets, ventral view, left. 189. ALS. 190. PMS. 191. PLS. Abbreviations are spelled out on p. 6.

RTA short, occupying about half tibial length, with distal end extended beyond tibia; lateral tibial apophysis large; cymbial furrow long, with distal end extended beyond cymbium, dorsal edge strongly concave; conductor moderately long; conductor dorsal apoph- 


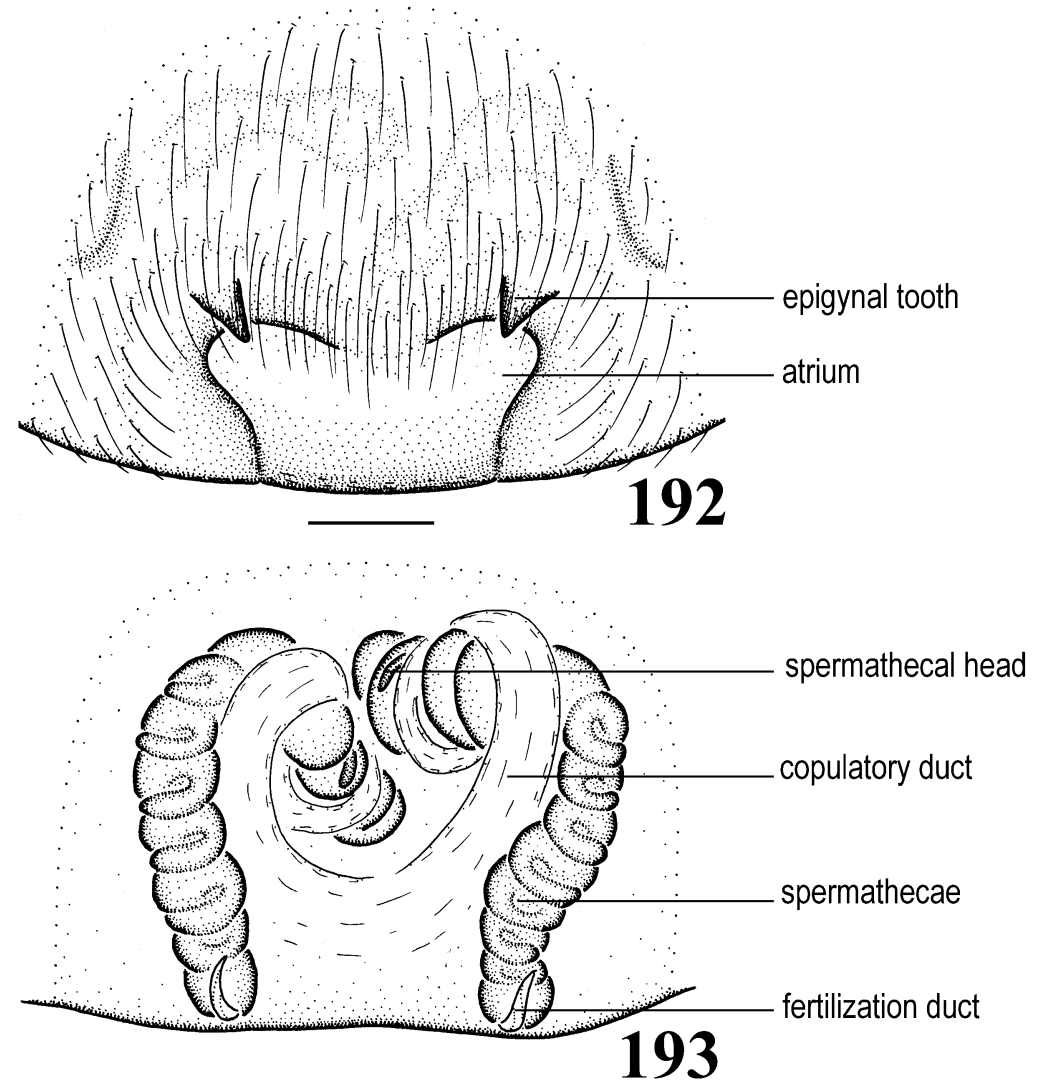

Figs. 192, 193. Draconarius sinualis (Chen, Zhao and Wang), female. 192. Epigynum. 193. Vulva.

ysis long; conductor lamella strongly developed; embolus posterior in origin, long, linear; median apophysis spoonlike, with distal end not sharply pointed (figs. 183-185).

Material ExAmined: CHINA: Hubei: Wudangshan, Jinding, August 1996, 1 male and 1 female paratype (J. Chen, HUW); Wudangshan, Jinding, September 24, 1997, 7 females (X. P. Wang, IZB); Wudangshan, Nanya to Jinding, September 24, 1997, 1 male and 16 females (X. P. Wang, IZB). Shaanxi: Taibaishan, Haoping, August 11, 1989, 1 male and 1 female, 1 male and 1 female, 1 male and 1 female, 1 male (X. P. Wang, AMNH and MCB); Taibaishan, Mingxinshi, Aug. 8, 1989, 1 female (X. P. Wang, IZB). Shanxi: Yongji, July 20, 1980, 1 female (M.S. Zhu, HUB, No-044).

Distribution: China (Hubei, Shanxi, Shaanxi).

\section{Draconarius sinualis (Chen, Zhao and Wang)}

Figures 192-210

Coelotes sinualis Chen, Zhao and Wang, 1991: 10, figs. 3, 4 (female holotype from Jinding, Wudangshan, Hubei, China, in HUW, examined); - Song et al., 1999: 378, figs. 224T, 224U.

DiAgnOSIS: Females can be distinguished by the strongly elongated, highly convoluted spermathecae, and the long, spiral copulatory ducts (fig. 192, 193), males by the long, slender conductor and the slender conductor dorsal apophysis (figs. 198, 199).

DESCRIPTION: Eye pattern similar to $D$. wudangensis. Chilum divided (fig. 200). Chelicerae with three promarginal and three retromarginal teeth (fig. 201). Epigynum with epigynal teeth short, widely separated, situated anteral to atrium; atrium small; cop- 



Figs. 194-197. Draconarius sinualis (Chen, Zhao and Wang), female spermathecae. 194. Dorsal view. 195. Ventral view. 196. Dorsal view, enlarged, showing spermathecal pores situated on spermathecae. 197. Ventral view, enlarged, showing spermathecal head pores.

ulatory ducts originated posteriorly, elongated, mesal to spermathecae; spermathecal heads small; spermathecae strongly elongated, highly convoluted, with indistinct stalks and bases (figs. 192-197).

Male patellar apophysis long; RTA short, occupying half tibia, with distal end extended beyond tibia; lateral tibial apophysis large; cymbial furrow strongly elongated, with distal end extended beyond cymbium, dorsal edge strong concave; conductor long, slender, slightly curved; conductor dorsal apophysis slender, long; conductor lamella strongly developed; embolus posterior in origin, extremely long, linear; median apophysis spoonlike, with distal end sharply pointed (figs. 198, 199).

Tarsal organ situated dorsally, away from distal tarsus, close to second distal trichobothrium (figs. 202-204). Apex of ALS with 2 major ampullate gland spigots (MAP), about 29 piriform gland spigots in female and 18 in male; PMS with 1 or 2 minor ampullate gland spigots in both sexes (not apparent), about 19 aciniform gland spigots in female and 12 in male, only 1 cylindrical gland spigot (CY) visible in female; PLS with about 20 aciniform gland spigots in both sexes and 2 cylindrical gland spigots in female (figs. 205-210).

Material Examined: CHINA: Hubei: Wudangshan, Jinding, April 23, 1982, female holotype (HUW); Wudangshan, Jinding, September 24, 1997, 3 females, 1 male and 2 females (X. P. Wang, AMNH); Wudangshan, Nanya to Jinding, September 24, 1997, 1 male and 1 female, 1 female, 11 females and 3 males (X. P. Wang, IZB); Wudangshan, Zhixiao to Nanya, September 23, 1997, 3 females and 7 males (X. P. Wang, MCB); Hongping, September 21, 1997, 2 males and 9 females (X. P. Wang, IZB). Shaanxi: Huxian, Cuihuashan, October 18, 1989, 1 male and 1 female (X. P. Wang, IZB).

Distribution: China (Hubei, Shaanxi).

\section{EUROCOELOTES, NEW GENUS}

TyPe Species: Coelotes inermis (L. Koch, 1855).

ETYMOLOGY: The generic name is derived from its similarity to Coelotes and from the occurrence in Europe, and is masculine in gender.

Diagnosis: Females resemble Coelotes but have posteriorly situated epigynal teeth, an large atrium, and large copulatory ducts (figs. 211, 212). Males also resemble Coelotes in having a conductor dorsal apophysis 




Figs. 198-201. Draconarius sinualis (Chen, Zhao and Wang). 198. Male palp, ventral view. 199. Male palp, retrolateral view. 200. Female, eyes, front view. 201. Female, chelicera, ventral view. 

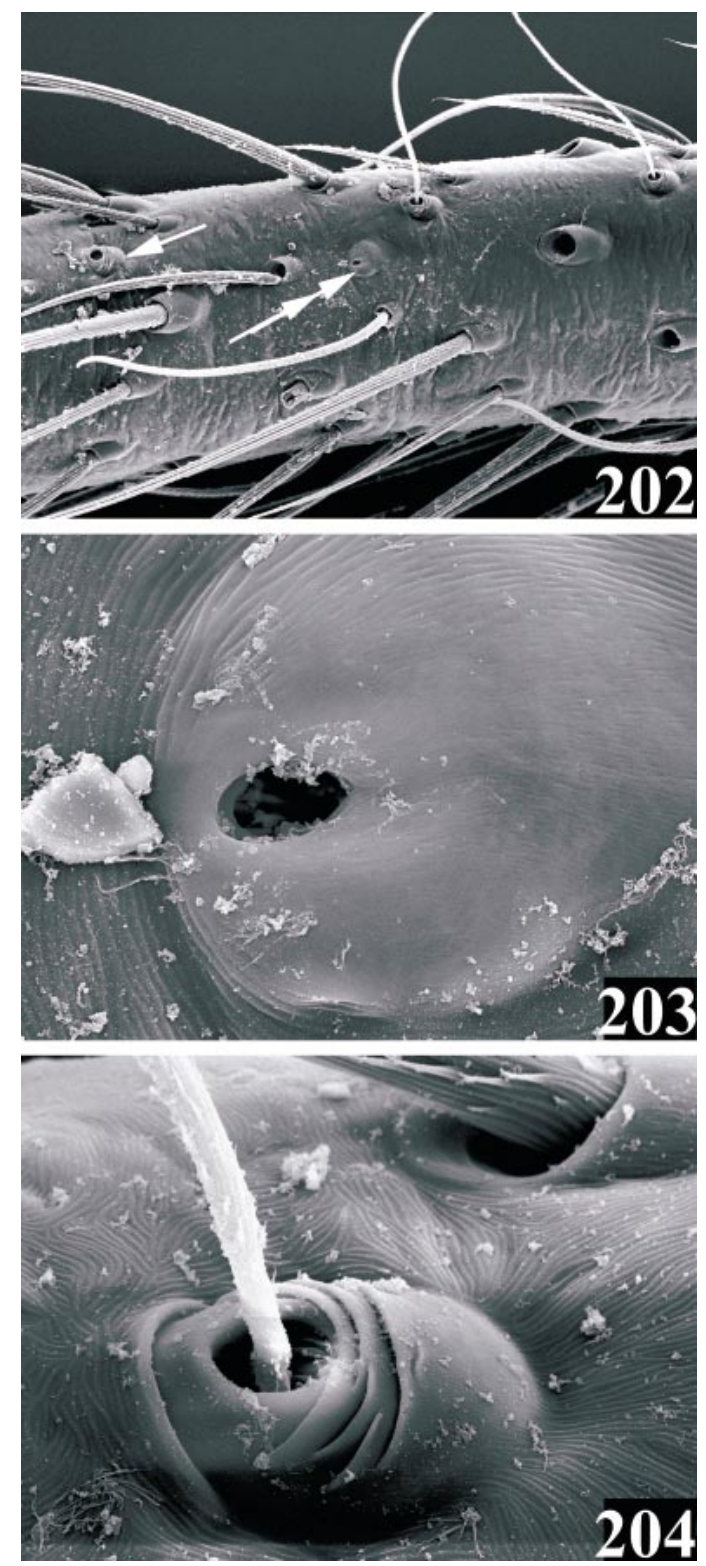

Figs. 202-204. Draconarius sinualis (Chen, Zhao and Wang), female. 202. Tarsus, showing position of tarsal organ (double arrows) and the distalmost trichobothrium (single arrow). 203. Tarsal organ. 204. Trichobothrium.

and short, rounded median apophysis, but have no patellar apophysis (figs. 213-216).

DESCRIPTION: See description of type species (below).

DisTRIBUTION: Europe (map 8).
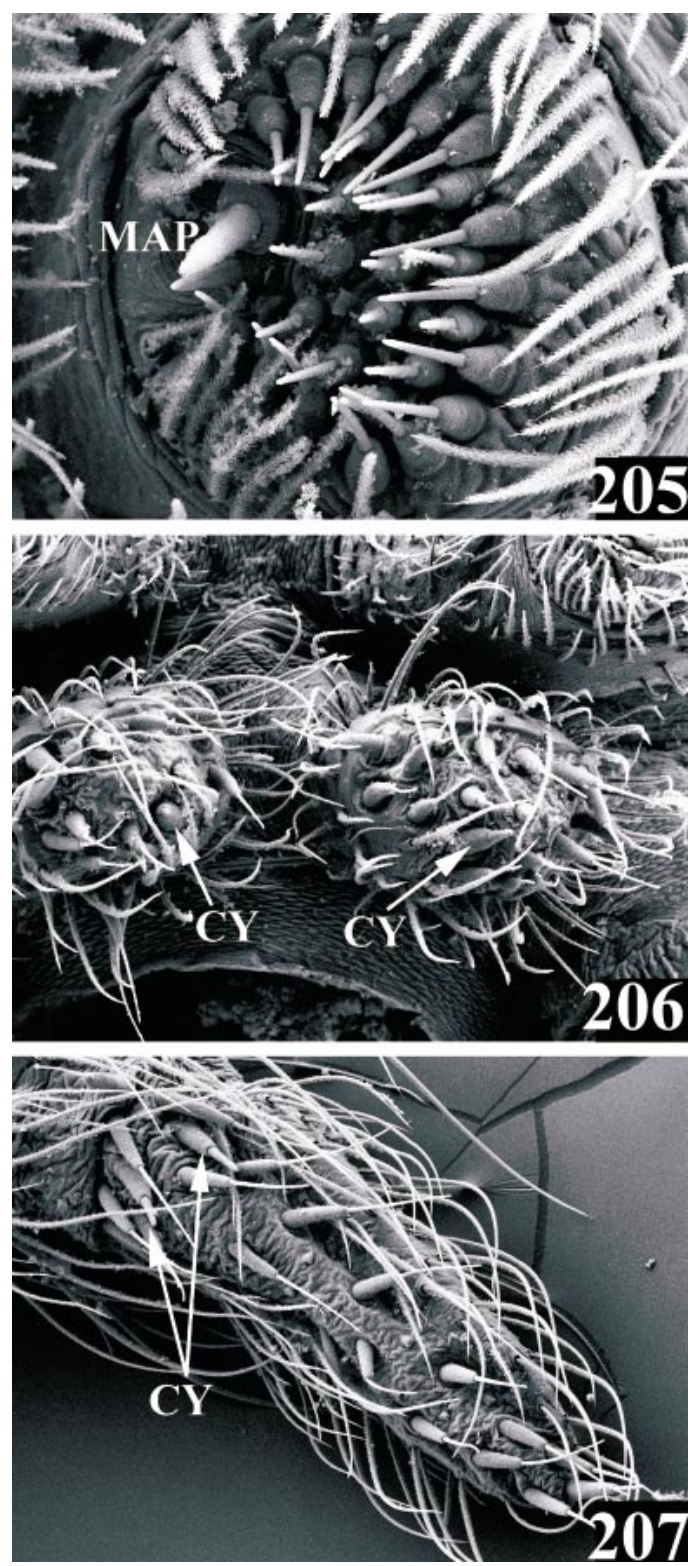

Figs. 205-207. Draconarius sinualis (Chen, Zhao and Wang), female spinnerets, ventral view, right. 205. ALS. 206. PMS. 207. PLS. Abbreviations are spelled out on p. 6.

Composition: Eleven species are included. All are new combinations.

1. Eurocoelotes anoplus (Kulczyn'ski, 1897): female lectotype from Croatia, Yugoslavia, in MNHN, examined). NEW COMBINATION (transferred here from the genus Coelotes). 

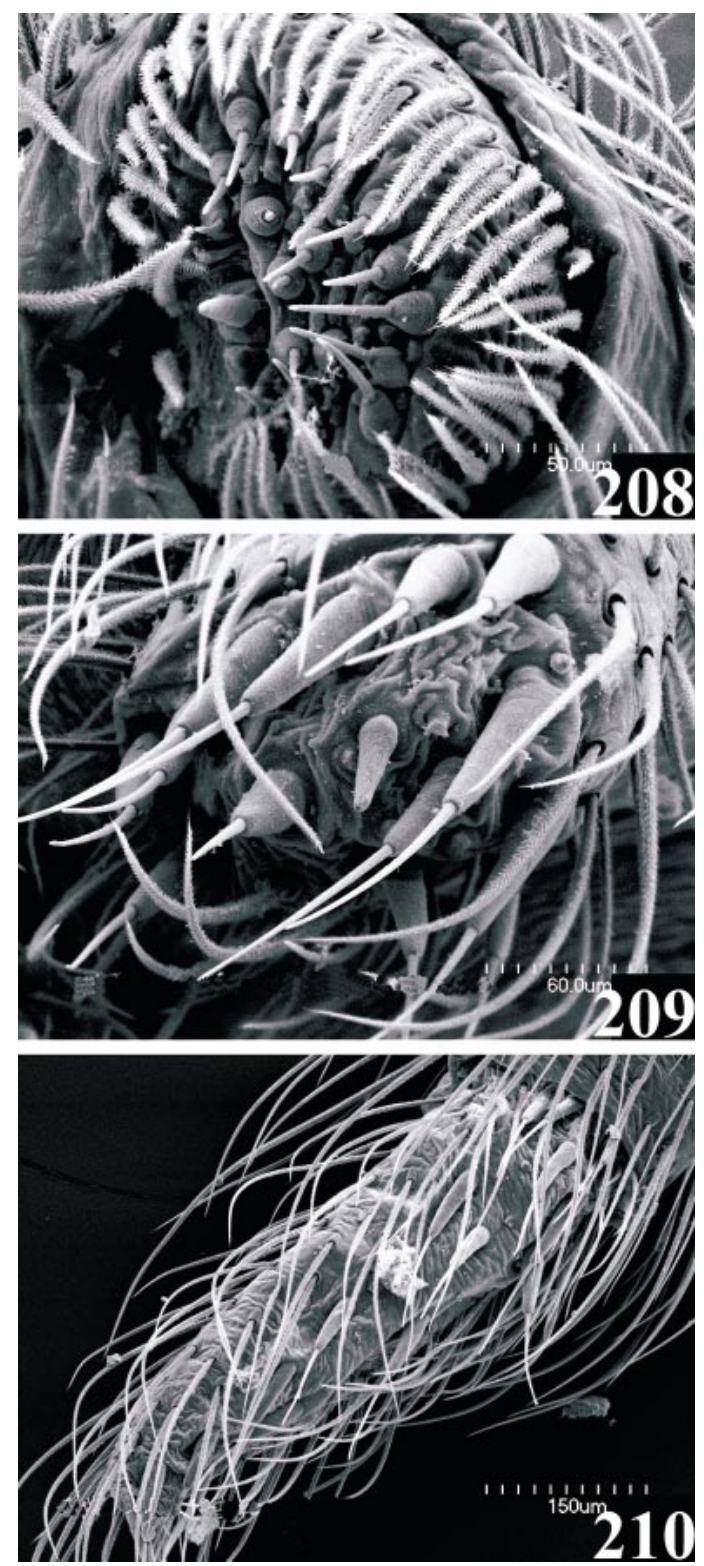

Figs. 208-210. Draconarius sinualis (Chen, Zhao and Wang), male spinnerets, ventral view, right. 208. ALS. 209. PMS. 210. PLS.

2. Eurocoelotes brevispinus (Deltshev and Dimitrov, 1996): male and female paratypes from Hambar dere, Slavyanka, Bulgaria, in IZS, examined. NEW COMBINATION (transferred here from Coelotes).

3. Eurocoelotes deltshevi (Dimitrov, 1993): male holotype, male and female par- atypes from Bulgaria, in IZS and Naturhistorisches Museum, Wien, not examined. NEW COMBINATION (transferred here from Coelotes).

4. Eurocoelotes drenskii (Deltshev, 1990): male holotype from Bulgaria, in IZS, not examined. NEW COMBINATION (transferred here from Coelotes).

5. Eurocoelotes falciger (Kulczyn'ski, 1897): types, depository unknown, not examined. NEW COMBINATION (transferred here from Coelotes). Male and female specimens from Bulgaria, in $\mathrm{MCB}, \mathrm{MCV}$, and $\mathrm{ZMB}$, examined.

6. Eurocoelotes gasperinii (Simon, 1891): male and female lectotypes from Dalmatia, Yugoslavia, in MNHN, examined. NEW COMBINATION (transferred here from Coelotes).

7. Eurocoelotes inermis (L. Koch, 1855): female neotype from Krakow, Poland, in SMNH, examined. NEW COMBINATION (transferred here from Coelotes).

8. Eurocoelotes jurinitschi (Drensky, 1915): types, depository unknown, not examined. NEW COMBINATION (transferred here from Coelotes). Male and female specimens from Bulgaria, in IZS, examined.

9. Eurocoelotes karlinskii (Kulczyn'ski, 1906): types, depository unknown, not examined. NEW COMBINATION (transferred here from Coelotes). Male and female specimens from East Europe, in IZS, examined.

10. Eurocoelotes kulczynskii (Drensky, 1915): types, depository unknown, not examined. NEW COMBINATION (transferred here from Coelotes). Male and female specimens from Bulgaria, in IZS, examined.

11. Eurocoelotes microlepidus de Blauwe, 1973: female holotype from Montecchio, Italy, in MCV, examined. NEW COMBINATION (transferred here from Coelotes).

\section{Eurocoelotes inermis (L. Koch) \\ Figures 211-226}

Amaurobius inermis L. Koch, 1855: 161, fig. 1 (female neotype from Krakow, Poland, in SMNH, examined). - Miller, 1971: 175, figs. $13-15$.

Coelotes inermis: L. Koch, 1868: 33, figs. 15, 16. - Simon, 1875: 45; - Kulczyn'ski, 1887: 341, fig. 57; - Becker, 1896: 189, fig. 1; - Chyzer and Kulczyn'ski, 1897: 157, fig. 16; - Bösenberg, 1902: 222, fig. 315; - Kulczyn'ski, 1906: 


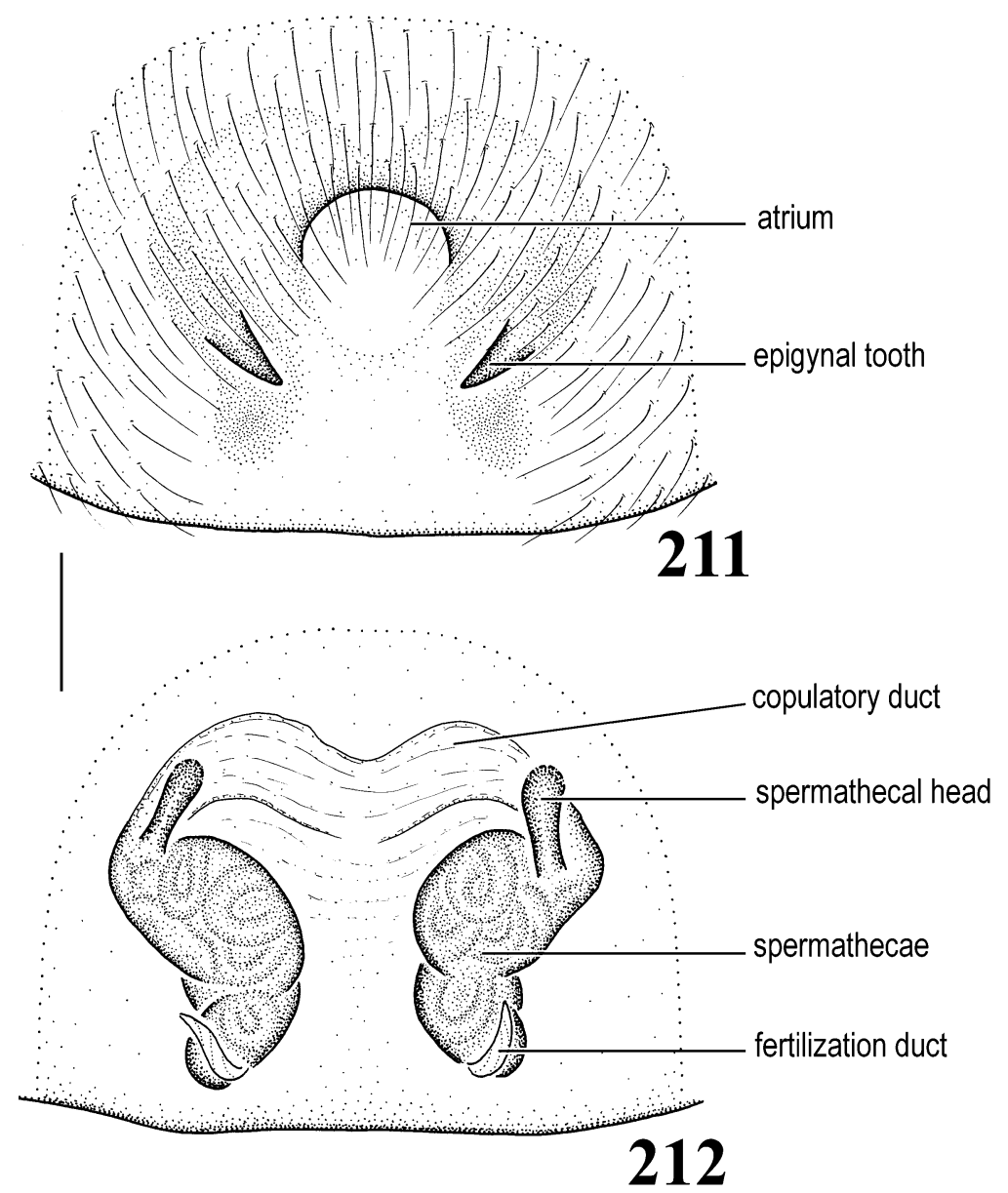

Figs. 211, 212. Eurocoelotes inermis (Koch), female. 211. Epigynum. 212. Vulva.

464, figs. 2, 59; - Dahl, 1931: 26, figs. 42, 43; - Simon, 1937: 983, 987, 1037, figs. 1508, 1516; - Drensky, 1942: 42, figs. 5k, 6a; - Loksa, 1969: 106, figs. 73D, 75B; - de Blauwe, 1973: 39, figs. 34-36; - Deltshev, 1990: 33, fig. 3; - Heimer and Nentwig, 1991: 356, fig. 925; - Roberts, 1995: 250; - Buchar et al., 1995: 120, fig. 35; - Ovtchinnikov, 1999: 74, figs. 31, 32.

Diagnosis: This species is similar to $E$. karlinskii but can be distinguished by the laterally originated, strong spermathecal heads of females (figs. 211, 212) and by the presence of a small tooth on the distal conductor and a broad conductor dorsal apophysis in males (fig. 213).

DESCRIPTION: Total length about 8.0010.5. General somatic characters same as Coelotes atropos. From front, anterior eye row slightly procurved, posterior row procurved; eye sizes and arrangements: AME smallest and slightly smaller than ALE, ALE subequal to PLE and PME; AME-AME subequal to AME-ALE, slightly shorter than AME diameter, ALE-PLE close together, PME-PLE slightly longer than PLE diameter, PME-PME slightly shorter than PME diameter, AME-PME slightly shorter than AME diameter. Clypeal height about twice AME diameter or slightly less, covered with long, strong setae; chilum divided, hairless, long (fig. 217). Chelicerae with three promarginal and three retromarginal teeth (fig. 218). Labium about as long as wide, slightly notched distally. Length of $1 \mathrm{st}$ leg patella + tibia shorter than carapace length.

Tarsal organ not seen on examined speci- 


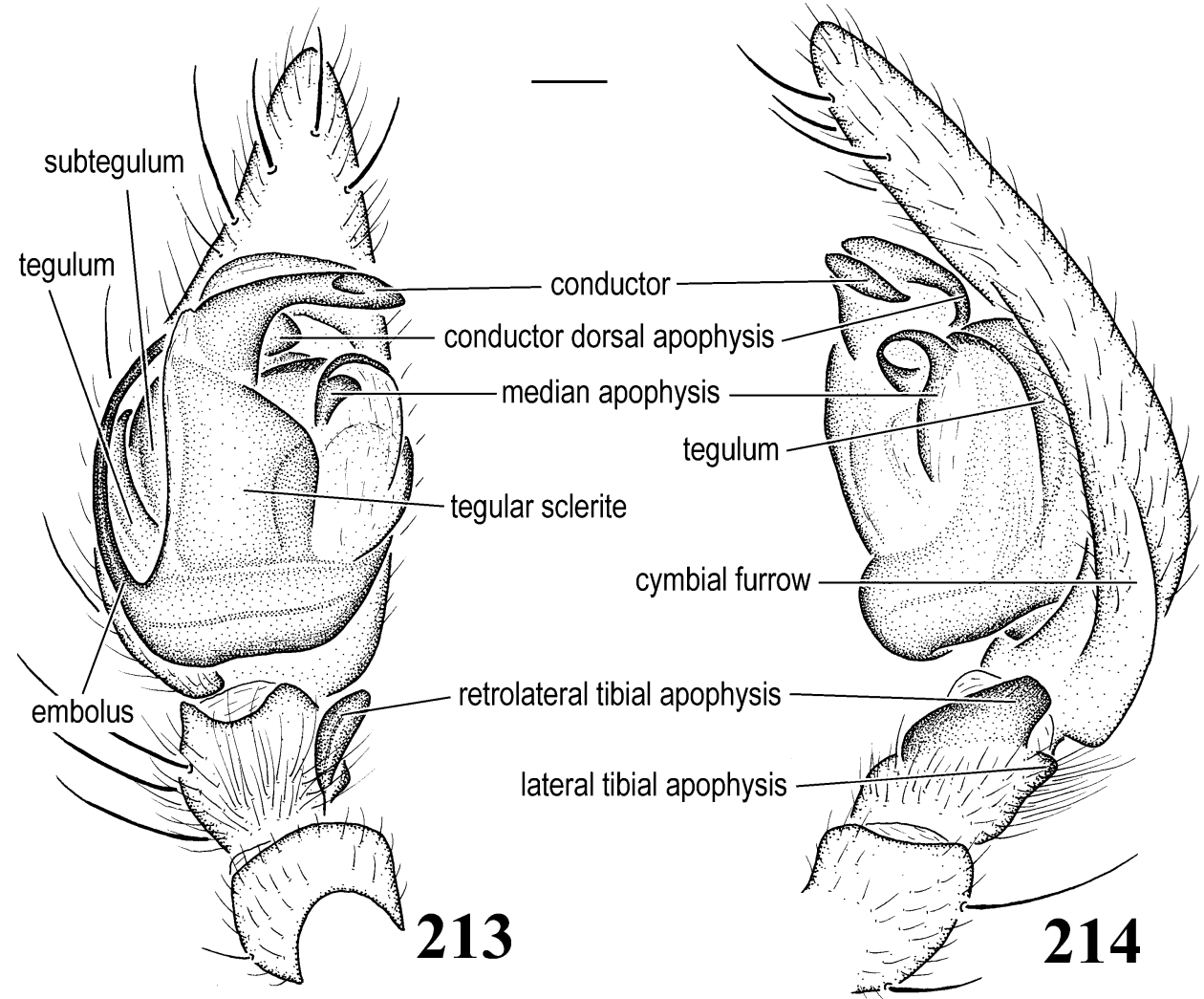

Figs. 213, 214. Eurocoelotes inermis (Koch), male palp. 213. Ventral view. 214. Retrolateral view, showing conductor dorsal view.

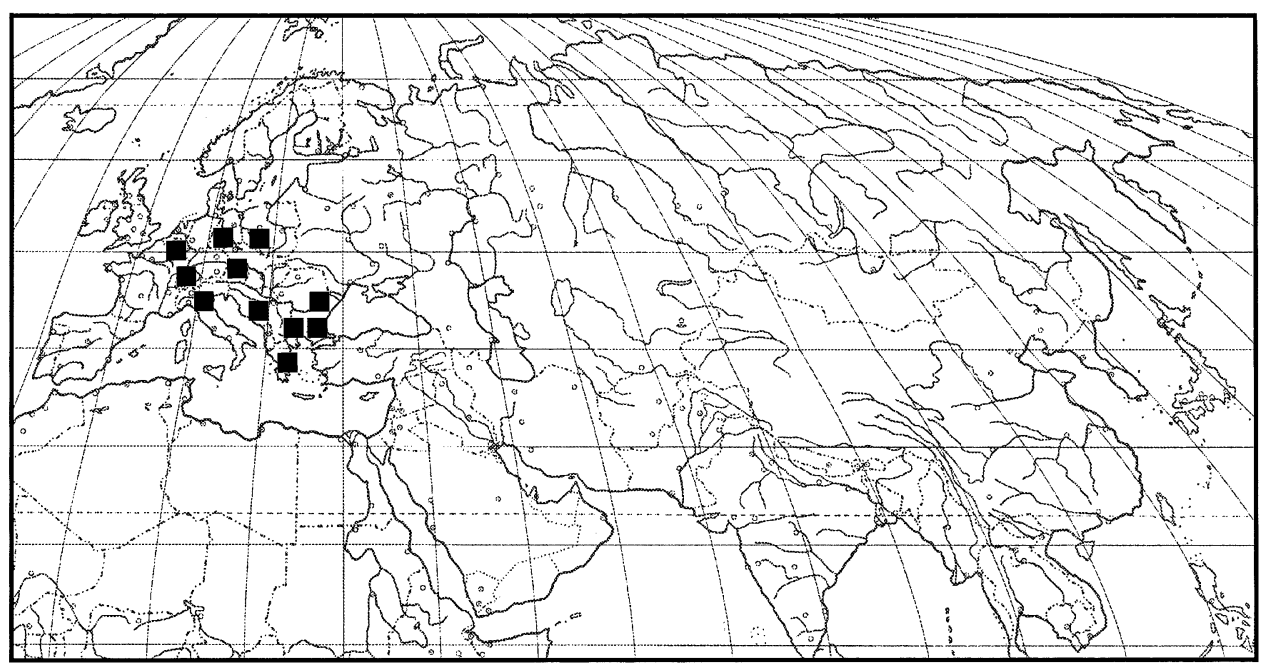

Map 8. Records of Eurocoelotes. 


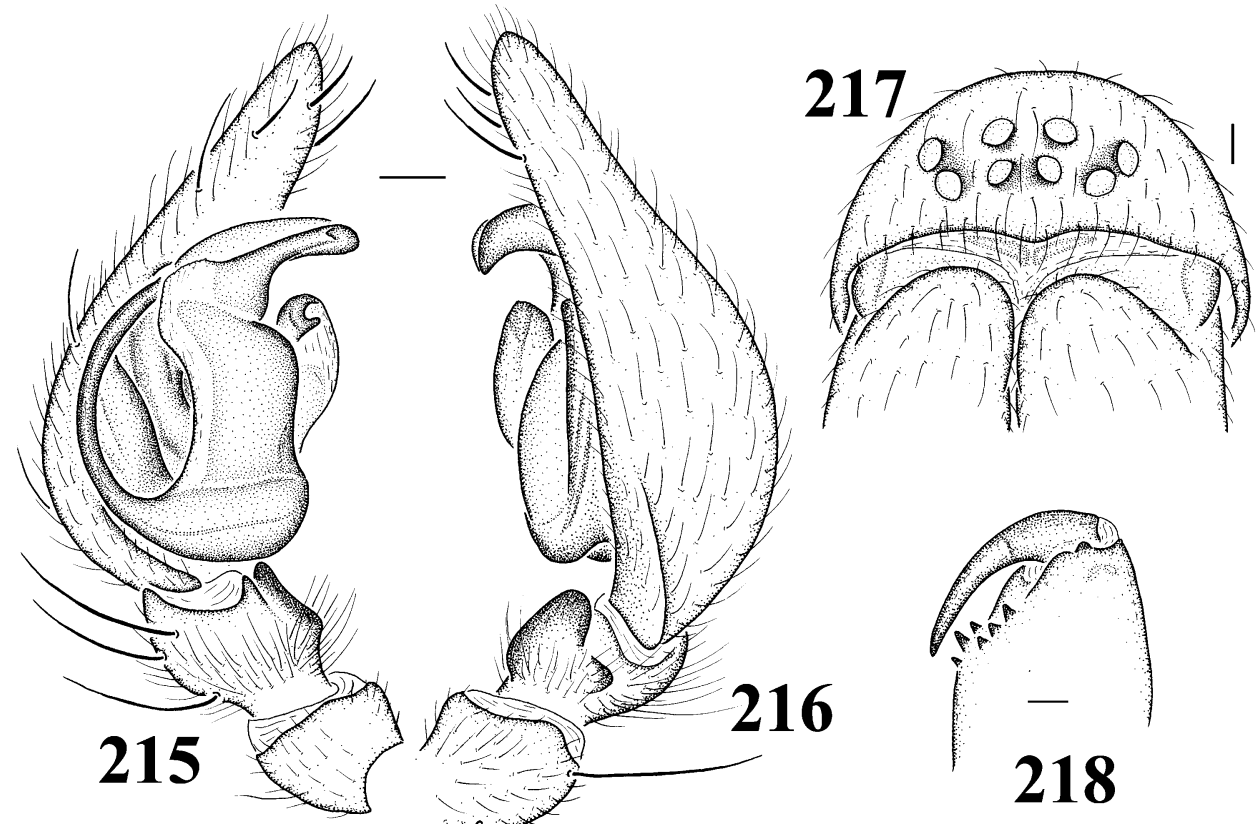

Figs. 215-218. Eurocoelotes inermis (Koch). 215. Male palp, prolateral view. 216. Male palp, retrolateral view. 217. Carapace, front view. 218. Chelicera, ventral view.

mens (figs. 219, 220). Apex of ALS with 2 major ampullate gland spigots (MAP), about 18-20 piriform gland spigots in both sexes; PMS with 1 or 2 minor ampullate gland spigots (mAP), one of them more or less reduced, about 43 aciniform gland spigots in female and at least 60 in male, 2 cylindrical gland spigots (CY) in female; PLS with about 60 aciniform gland spigots in female and about 78 in male, and 2 cylindrical gland spigots in female (figs. 221-226).

Epigynum with epigynal teeth short, situated posterad of atrium; atrium small, anteriorly situated; copulatory ducts broad, membranous, anteriorly situated; spermathecal heads long, situated anterad of spermathecae; spermathecae longitudinally elongated, convoluted, widely separated, with indistinct bases and stalks (figs. 211, 212). Male palp with patellar apophyses absent; RTA large, with distal end strongly extended beyond tibia; lateral tibial apophysis present; cymbial furrow short; tegular sclerite apparent; conductor strong, with small tooth near apex; conductor dorsal apophysis broad; conductor lamella moderately developed; embolus basal to prolateral in origin, moderately long, linear, relatively strong; median apophysis spoonlike, with smooth distal end, not pointed (figs. 213-216).

MATERIAL EXAMINED: AUSTRIA: Graz, September 1875, 1 male (H. Emerton, MCZ). BULGARIA: C. Balkan, C. Tuzha, 1500 m, August 10, 1996, 2 males (C. Deltshev, IZS). FRANCE: 2 females (MCZ). ITALY: Friuli-Venezia Giulia, Arta Terme, 440 m, September 7, 1963, 1 female (Bianchi Valle, MCB); Friuli-Venezia Giulia, Paluzza, 600 m, September 9, 1963 (Bianchi Valle, MCB); Bolzano, Planca di Sotto, August 25, 1972, 1 female (Rallo, MCV); Bressanone, Tonte Plose, $1200 \mathrm{~m}$, June 18, 1972, 1 female (Oppi, MCV); Basel, 1 male and 1 female (AMNH). GERMANY: Nurnberg, 1 male and 1 female (SMNH, Coll. Thorell, 227/ 1383c); between Deutz and Siegen, August 9, 1964, 1 female (USNM); 1 male (HEC, B.438, t.100); Neiderwall a Rhein, 1 female (CAS). POLAND: Krakow, 1 female neotype (SMNH, Coll. Thorell, 227/1383a); Roztocze Natl. Pk., Bukowa Gora, June 20, 1987, 3 females (B. and H. Malkin, AMNH). Pachow, Pow. Wadowice. Woj. Krakowskie, September 8, 1974, 1 female (B. Malkin and 

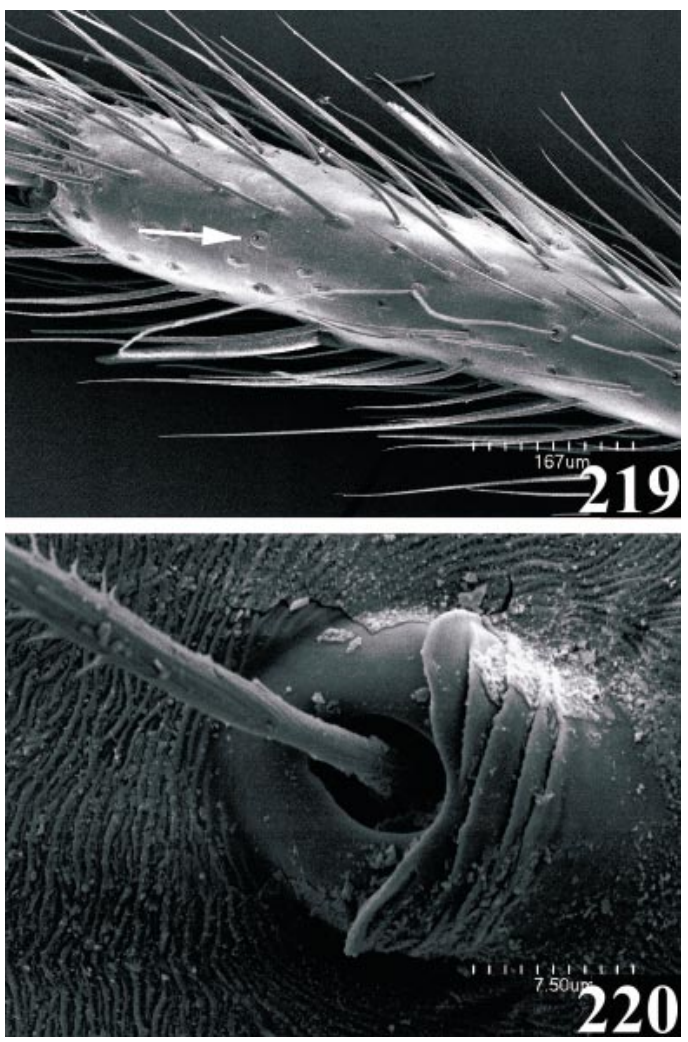

Figs. 219, 220. Eurocoelotes inermis (Koch), female. 219. Tarsus, with tarsal organ not visible (single arrow shows trichobothrium). 220. Trichobothrium.

M. Mlynarski, AMNH). SWITZERLAND: Umgebung Basel, Franmatt VII, 6 males and 17 females (Keine Angaben, NHMB); Alpes, 1 male and 1 female (SMNH, Coll. Thorell, 227/1383b); Basel, 1 male and 1 female (Schenkel, AMNH); Predigerholz, SW of Neumunchenstein, 340 m, under bark of oak log, September 2, 1973, 1 female (B. Malkin and H. and I. Hurlimann, AMNH). Solothurn, Oensingen-Schloss, June 13, 1980, 1 female (B. and H. Malkin, AMNH); Ruttenen, May 1-2, 1980, 2 males (B. Malkin, AMNH); May 1976, 1 female (B. Malkin, AMNH); Oberdorf, August 1973, 1 female (B. Malkin, AMNH). YUGOSLAVIA: Fra Gospic e Karlobag, August 17, 1969, 1 male and 3 females (Bianchi Valle, MCB). Passo di Kupras, August 11, 1970, 1 male (MCB); August 11, 1970, 1 male (Mc Brigamo, MCV). Slavenia, Kranj Forest, 1960, 3 males
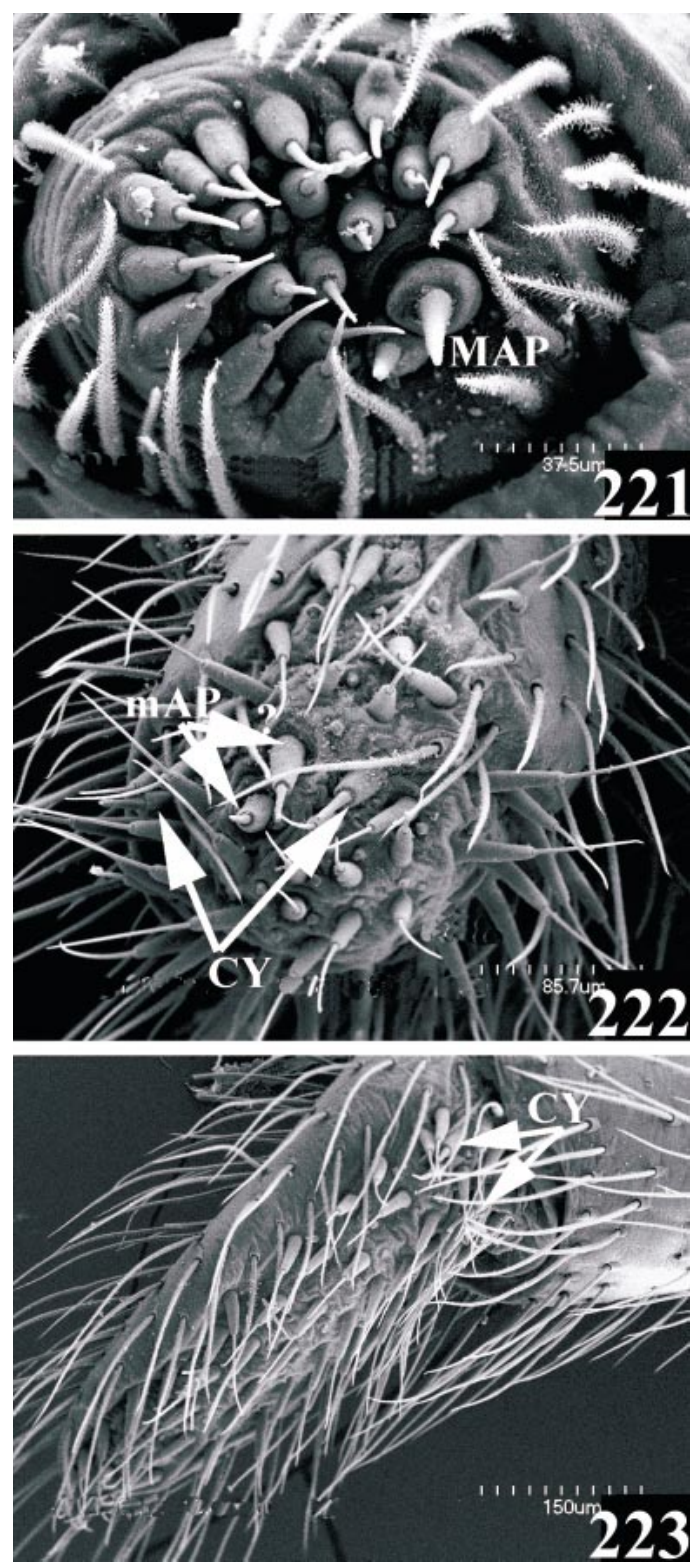

Figs. 221-223. Eurocoelotes inermis (Koch), female spinnerets, ventral view, left. 221. ALS. 222. PMS. 223. PLS. Abbreviations are spelled out on p. 6.

and 3 females (A. Polenec, MCZ). EUROPE: 2 males (AMNH-CU, Lot. 581, Sub. 499). 1 male and 2 females (Marx Collection, USNM, No. 242); Seyde Osterzgeb, Vienweide, November 21, 1967, 1 female (ZMB, Kat.-Nr. 28823); 1 male, 2 females, 1 female, 

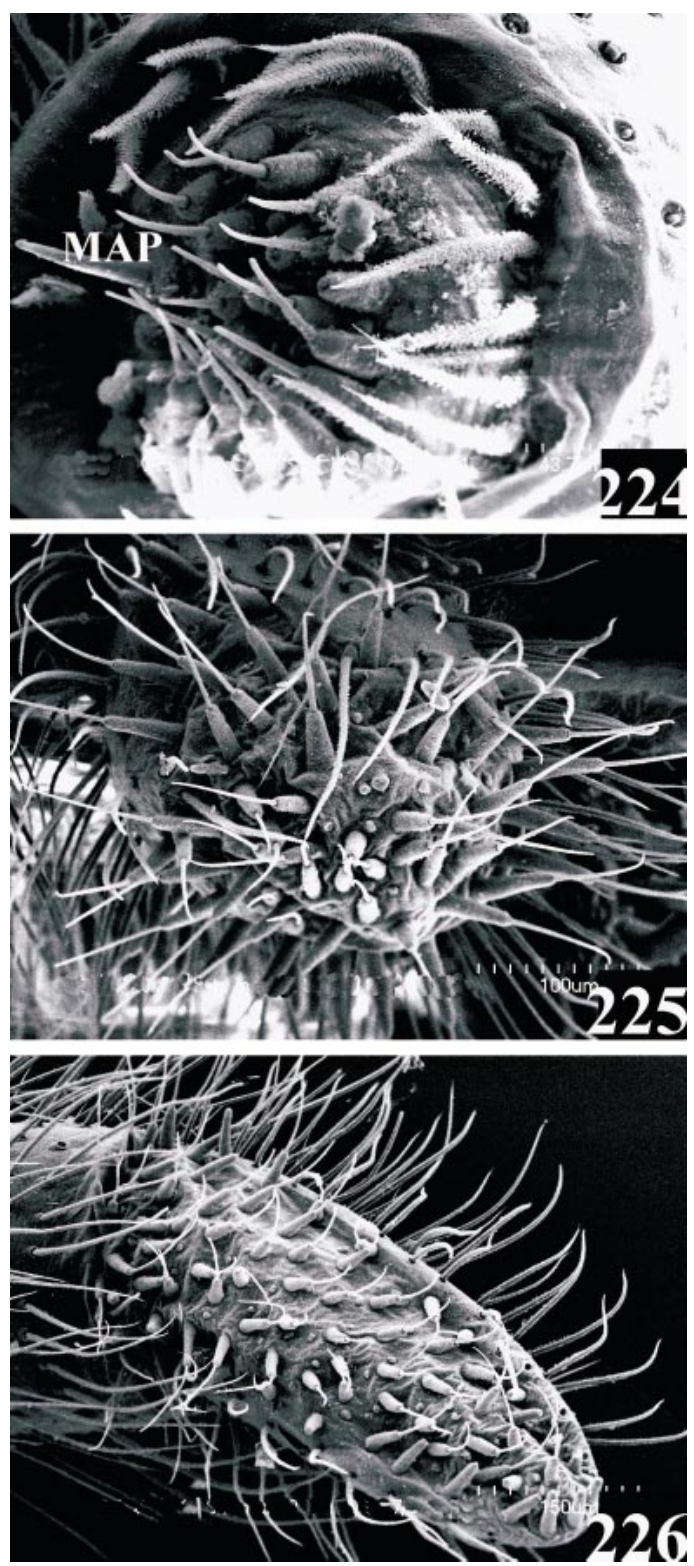

Figs. 224-226. Eurocoelotes inermis (Koch), male spinnerets, ventral view, right. 224. ALS. 225. PMS. 226. PLS. Abbreviations are spelled out on p. 6.

1 male, 3 females, 2 females, 1 male and 1 female, 1 female (ZMB); 2 females (ZMB, Dahl 1162); 3 males (ZMB, Kat.-Nr. 14016); 1 male and 1 female (ZMB, 5169); 8 males (ZMB, Kat.-Nr. 14094); 1 male and 1 female (ZMB, 4698); 2 males and 4 females (ZMB,
14473); 4 males (ZMB, Kat.-Nr. 14013); 1 male and 1 female (ZMB, Dahl 2073); Tharaudt, F. Fichtenwald, 3 males and 1 female (ZMB); 1 male and 2 females (ZMB, 4696); 1 male (ZMB, Dahl 877); 1 male (ZMB, 14478); 1 female (ZMB, Dahl 928); 3 males (ZMB, 14471); 2 males (ZMB, 14475); 1 female (ZMB, Dahl, 926); 1 female (ZMB, 1218); 1 female (ZMB, Dahl 1636); 1 male (ZMB, Dahl 2120); 1 female (ZMB, Dahl 2358); 1 female (ZMB, Dahl 1343); 1 female (ZMB, 14480); 6 males (ZMB, 14018); 1 male (ZMB, 14480); 6 males (ZMB, 14018); 1 male (ZMB, 14470); 1 female (ZMB, Dahl 2074); 1 female (ZMB, Dahl 2119); 2 males (ZMB, 14476); 2 females (ZMB, Dahl, 2135); 1 male (ZMB, 14477); 1 male (ZMB, 14474); 4 males (ZMB, 14472).

Distribution: Bulgaria, France, Germany, Italy, Poland, Switzerland, Yugoslavia.

\section{FEMORACOELOTES, NEW GENUS}

TyPE SPECIES: Coelotes platnicki Wang and Ono, 1998.

ETYMOLOGY: The generic name is derived from Coelotes and the special femoral characteristics of the male palp (the presence of distal apophysis and of a cluster of tiny setae prolaterally), and is masculine in gender.

DiAGNOSIS: Females of this genus can be easily recognized by the absence of epigynal teeth and the broad copulatory ducts (figs. 227,228 ), males by the presence of a femoral apophysis and the absence of a median apophysis (figs. 229-231).

DESCRIPTION: See description of type species (below).

DisTRIBUTION: China (map 9).

Composition: Two species are included in this genus.

1. Femoracoelotes latus (Wang, Tso, and $\mathrm{Wu}, 2001)$ : male holotype, male and female paratypes from Nantou, Taiwan, in THU, examined. NEW COMBINATION (transferred here from Coelotes).

2. Femoracoelotes platnicki (Wang and Ono, 1998): male holotype and female paratype from Mt. Tengchih, Paoshan-tsun, Taoyuan-hsiang, Kaohsiung-hsien, Taiwan, in NSMT, examined. NEW COMBINATION (transferred here from Coelotes). 


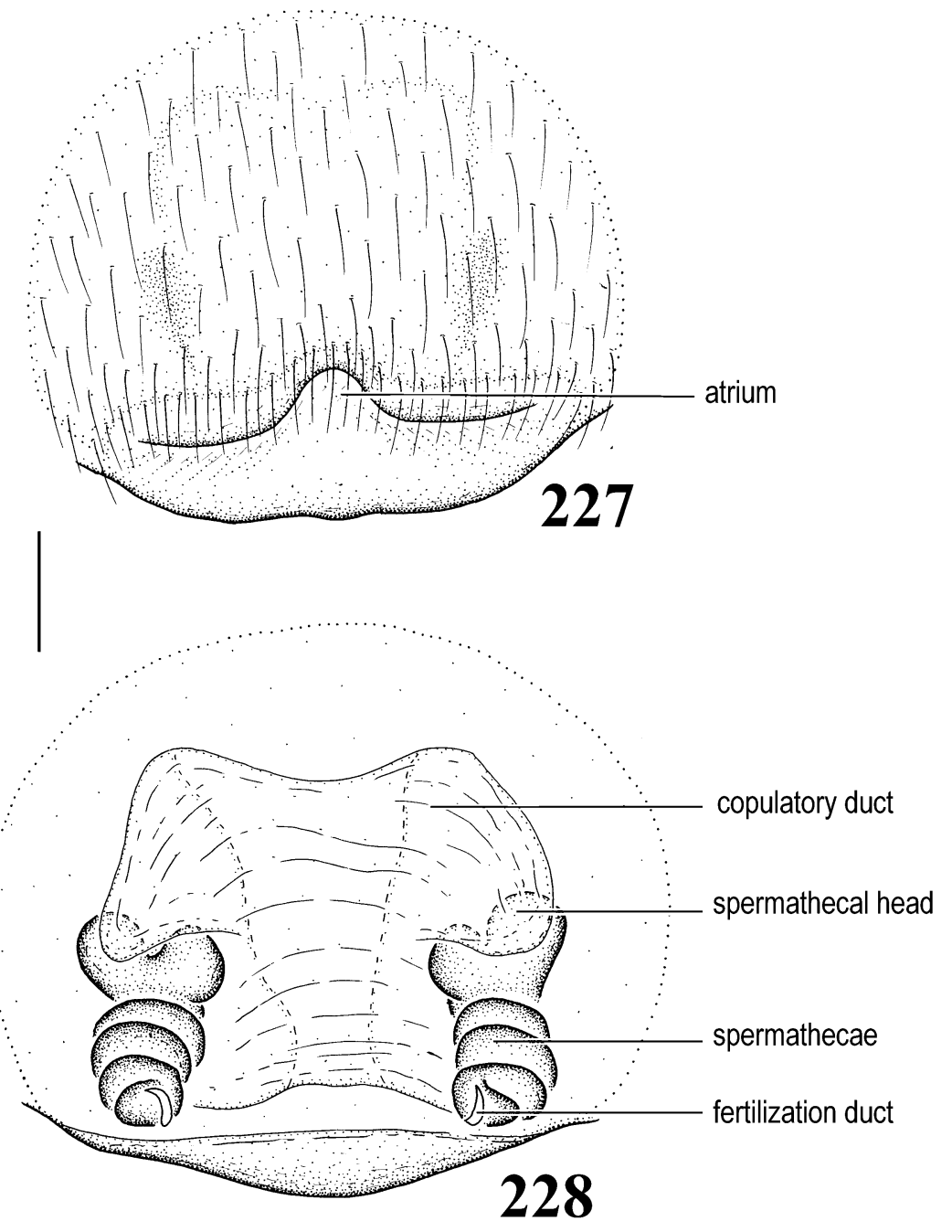

Figs. 227, 228. Femoracoelotes platnicki Wang and Ono, female. 227. Epigynum. 228. Vulva.

Femoracoelotes platnicki (Wang and Ono)

Figures 227-241

Coelotes platnicki Wang and Ono, 1998: 148, figs. 15-19 (male holotype and female paratype from Mt. Tengchih, Paoshan-tsun, Taoyuanhsiang, Kaohsiung-hsien, Taiwan, in NSMT, examined). - Song et al., 1999: 377.

Diagnosis: Differs from $F$. latus in the shape of the atrium and the broad copulatory ducts of females (figs. 227, 228) and by the slightly bifid femoral apophysis and the different conductor shape of males (figs. 229231).

Description: Total length 6.00-8.00. From front, anterior eye row slightly procur- ved, posterior row procurved; eye sizes and arrangements: AME smallest, PLE largest, PME slightly smaller than PLE, ALE slightly smaller than PME; AME-AME subequal to AME-ALE, ALE-PLE close together, PMEPLE separated by roughly PME diameter or slightly less, PME-PME subequal to AMEAME or slightly longer. Clypeal height roughly 1.5 AME diameter; chilum divided, elongated (fig. 232). Chelicerae with three promarginal and four retromarginal teeth (occasionally five) (fig. 233). Labium with length subequal to width. Length of female 1 st leg patella + tibia shorter than carapace length. 


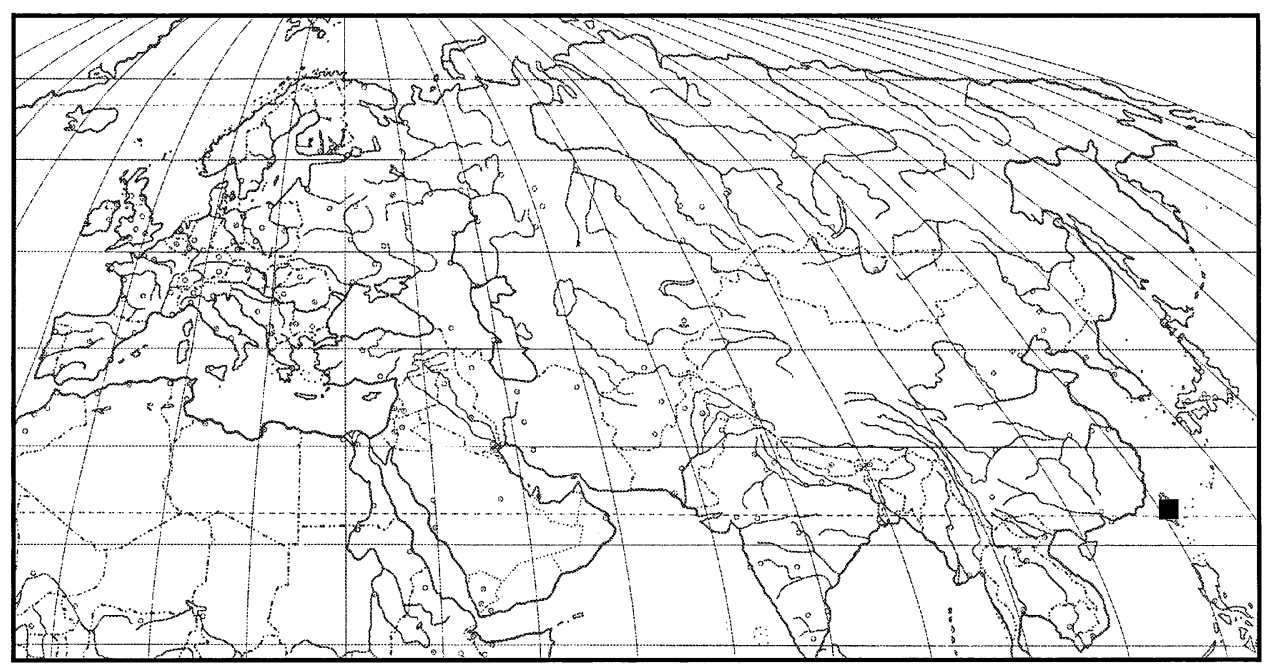

Map 9. Records of Femoracoelotes.

Tarsal organ situated away from distal end of tarsus, close to third distalmost trichobothrium (figs. 234, 235). Apex of ALS with 2 major ampullate gland spigots (MAP), about 24-25 piriform gland spigots in both sexes; PMS with 1 or 2 minor ampullate gland spigots (mAP) in both sexes, about 16-
18 aciniform gland spigots in both sexes, 2 cylindrical gland spigots (CY) in female; PLS with about 16 aciniform gland spigots in female and 21 in male, 2 cylindrical gland spigots in female, 1 spigot of examined male PLS also shaped like cylindrical gland spigot (figs. 236-241).



Figs. 229, 230. Femoracoelotes platnicki Wang and Ono, male palp. 229. Ventral view. 230. Retrolateral view. 

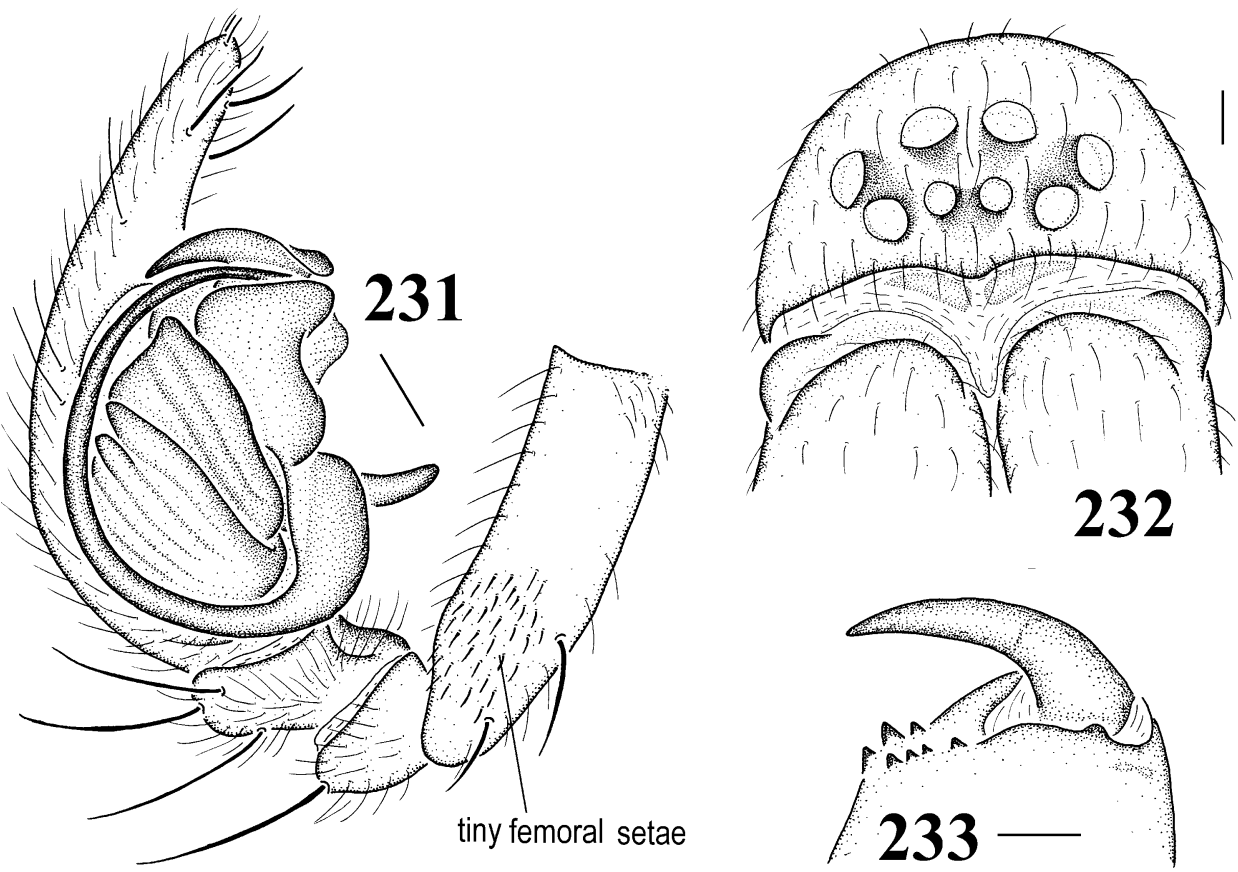

Figs. 231-233. Femoracoelotes platnicki Wang and Ono. 231. Male palp, prolateral view. 232. Carapace, front view. 233. Chelicera, ventral view.

Epigynum without epigynal teeth; atrium relatively small; copulatory ducts broad, membranous; spermathecal heads apparent; spermathecae short, convoluted, widely separated, with indistinct stalks and bases (figs. 227, 228). Male palp with femoral apophysis distally, a cluster of tiny setae prolaterally; patellar apophysis short, ventrally curved; RTA almost as long as tibia, with distal end not extended beyond tibia; lateral tibial apophysis absent; cymbial furrow short; conductor broad, complex, strongly modified; conductor lamella small; conductor dorsal apophysis relatively ventrally situated, long, slender; median apophysis absent; embolus basal in origin, strong, moderately long, with apex modified (figs. 229-231).

Material Examined: CHINA: Taiwan: Kaohsiung-hsien, Taoyuan-hsiang, Paoshantsun, Mt. Tengchih, 1550-1800 m alt., November 1, 1989, 5 males and 7 females $(H$. Ono, NSMT, NSMT-Ar.3421); Kaohsiunghsien, Taoyuan-hsiang, Paoshan-tsun, Mt. Tengchih, $1550 \mathrm{~m}$ alt., November 1, 1989, 1 male (H. Ono, NSMT, NSMT-Ar.3430); Kaohsiung-hsien, Taoyuan-hsiang, Paoshan-tsun,
Mt. Tengchih, 1550-1800 m alt., November 1, 1989, male holotype and female paratype (H. Ono, NSMT, NSMT-Ar.3421).

Distribution: China (Taiwan).

\section{HIMALCOELOTES, NEW GENUS ${ }^{1}$}

Type Species: Himalcoelotes martensi, new species.

ETYMOLOGY: The generic name refers to its similarity to Coelotes and to the locality of type species, and is masculine in gender.

DiAGNOSIS: Females resemble those of Urocoras in having elongated epigynal teeth, but differ in having short copulatory ducts (fig. 265, 266); males resemble those of Draconarius in having the patellar apophysis and the conductor dorsal apophysis, but differ in the shape of the median apophysis (small, rounded, medially notched and then strongly extended) (figs. 244, 267, 268).

\footnotetext{
${ }^{1}$ Results of the Himalaya Expeditions of J. Martens, no. 239. For no. 238, see Courier Forschungsinstitut Senckenberg 232: 1-136, 2001. Note: J. Martens was sponsored by Deutscher Akademischer Austauschdienst and Deutsche Forschungsgemeinschaft.
} 

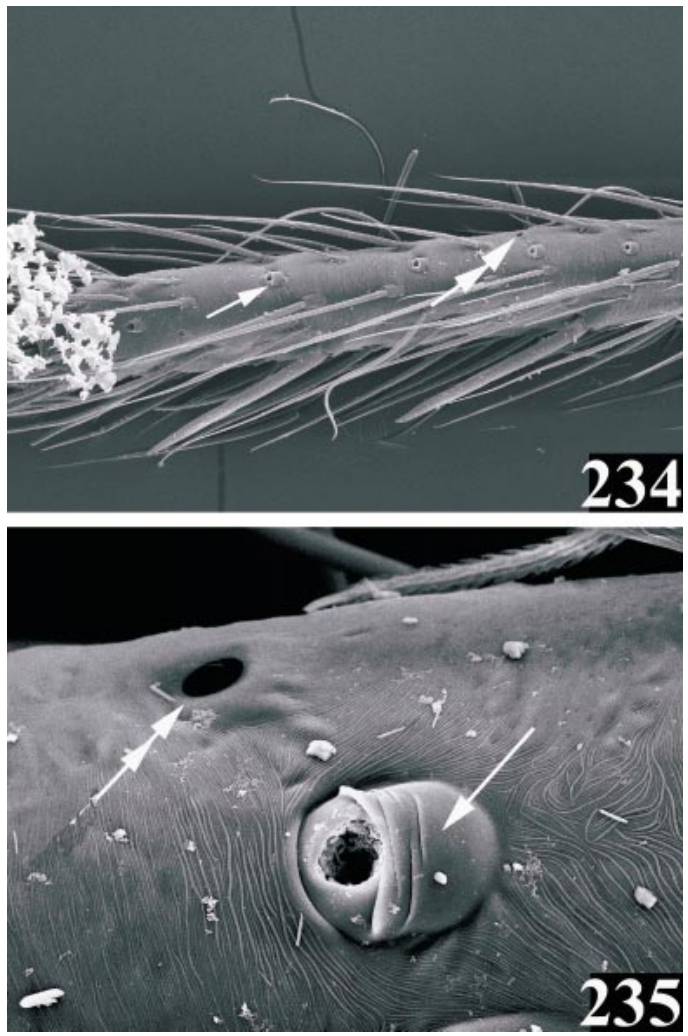

Figs. 234, 235. Femoracoelotes platnicki Wang and Ono, female. 234. Tarsus, showing position of tarsal organ (double arrows) and the distalmost trichobothria (single arrow). 235. Tarsal organ (double arrows) and third distal trichobothrium (single arrow).

DESCRIPTION: See description of type species (below).

DistRIBUTION: Himalayas (map 10).

Composition: Ten species are included.

\section{Key to Species of Himalcoelotes}

\section{Females}

1. Spermathecae small, rounded, not convoluted (figs. 249, 283, 293) .......... 2

- Spermathecae otherwise (figs. 243, 266) . . . $\ldots \ldots \ldots \ldots \ldots \ldots$

2. Copulatory ducts broad, situated mesad of spermathecae (fig. 249) ..... . bursarius

- Copulatory ducts not visible or barely visible in dorsal view (figs. 283, 293) . . . . . 3

3. Anterior atrial margin incomplete; copulatory ducts not seen (figs. 282-287) . . sherpa

- Anterior atrial margin complete; copulatory ducts small (figs. 292, 293) ... subsherpa
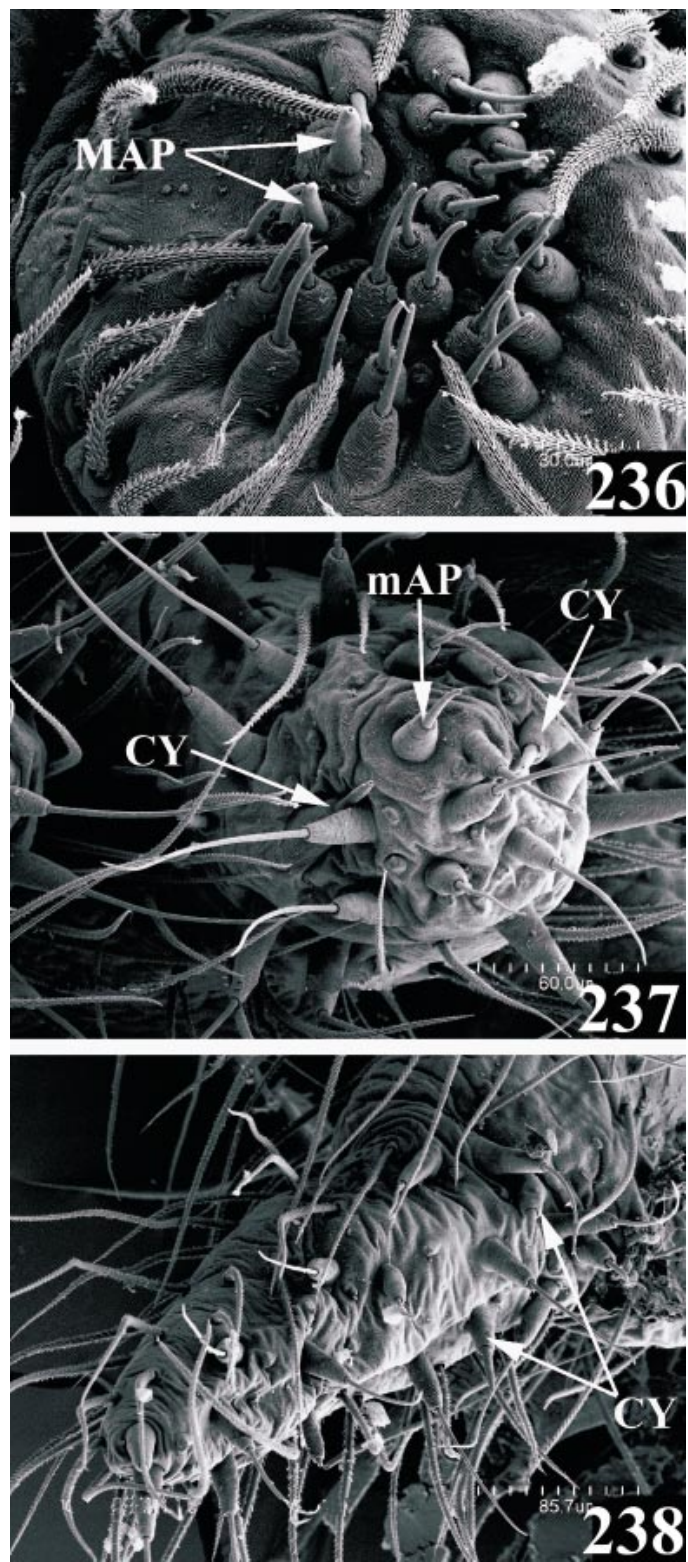

Figs. 236-238. Femoracoelotes platnicki Wang and Ono, female spinnerets, ventral view, right. 236. ALS. 237. PMS. 238. PLS. Abbreviations are spelled out on p. 6.

4. Spermathecal large, anteriorly extended; spermathecal heads situated mesally or posteriorly (figs. 255,266 ) . . . . . . . . 5

- Spermathecal moderately large, not anteriorly extended; spermathecal heads situated anteriorly (figs. $243,251,279) \ldots \ldots .6$

5. Spermathecal heads situated mesally; copu- 

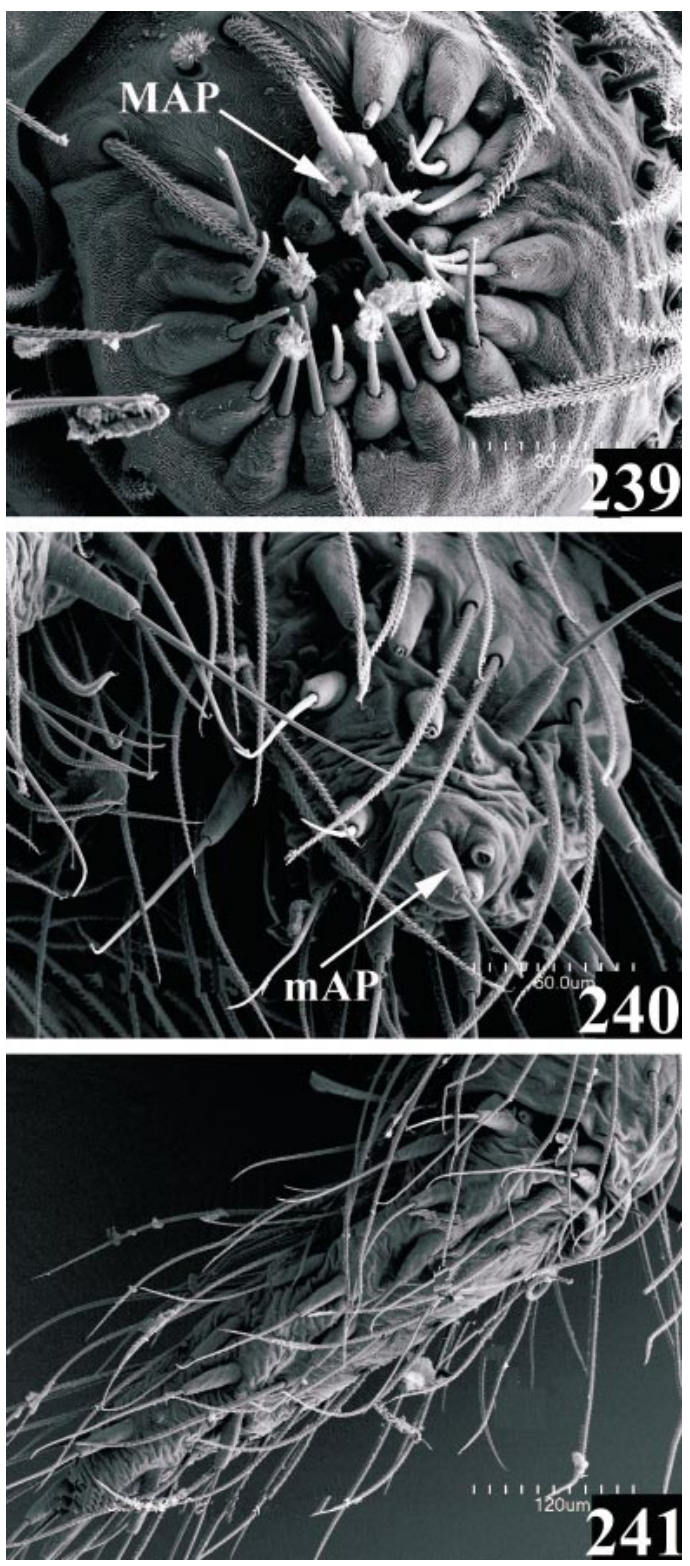

Figs. 239-241. Femoracoelotes platnicki Wang and Ono, male spinnerets, ventral view, right. 239. ALS. 240. PMS. 241. PLS. Abbreviations are spelled out on p. 6.

latory ducts not seen in dorsal view (fig. 266) ................ martensi

- Spermathecal heads situated posteriorly; copulatory ducts originated laterally (fig. 255) $\ldots \ldots \ldots \ldots \ldots$ gyirongensis

6. Spermathecae strongly curved, semicircleshaped (figs. 243, 295) ......... 7
- Spermathecae longitudinally extended, not curved (figs. 247, 253, 279) . . . . . .8

7. Epigynal teeth long, slender; copulatory ducts short, not visible in dorsal view (figs. 242, 243) ............... aequoreus

- Epigynal teeth short, broad; copulatory ducts visible in dorsal view (figs. 294, 295) ... .................. syntomos

8. Atrium apparent; copulatory ducts visible in dorsal view (figs. 250, 251, 278, 279) ...

- Atrium reduced to atrial slit; copulatory ducts not visible in dorsal view (figs. 246, 247) ................. brignolii

9. Spermathecae separated, copulatory ducts extended mesally (figs. 251, 253) ...... $\ldots \ldots \ldots \ldots \ldots \ldots$ diatropos

- Spermathecae close together, copulatory ducts extended anteriorly (fig. 279) ... ................. pirum

Males

1. Cymbial furrow more than half of cymbial length (figs. 245, 258) . . . . . . . . 2

- Cymbial furrow less than half of cymbial length (figs. 268, 289, 281, 291) .... . 3

2. RTA strongly extended anteriorly; patellar apophysis curved ventrally (fig. 258) .... ................ gyirongensis

- RTA slightly extended; patellar apophysis curved dorsally (fig. 245) ... A Aequoreus

3. Embolus prolateral in origin (figs. 267,288 ) $\ldots \ldots \ldots \ldots \ldots \ldots \ldots \ldots$

- Embolus basal in origin (figs. 280, 290) ... ..................... 5

4. Conductor broad; patellar apophysis much shorter than patellar length (figs. 267, 268) ................. martensi

- Conductor slender; patellar apophysis broad, as long as patellar length (figs. 288, 289)

sherpa

5. Conductor with apex broad, no tooth (figs. $280,281) \ldots \ldots \ldots \ldots \ldots \ldots$ pirum

- Conductor with apex slender, with small tooth (figs. 290, 291) . ......... subsherpa

Himalcoelotes aequoreus, new species Figures 242-245

TYPE: Female holotype (SMF, no.24) from forest clearing, Thaksang above Tukche, 3100-3300 m, Mustang Dist., Nepal (July 510, 1970; J. Martens); male paratype (SMF, no.18) from Tukche, in house, $2600 \mathrm{~m}$, Mustang Dist., Nepal (October 1969; J. Martens), deposited in SMF. 


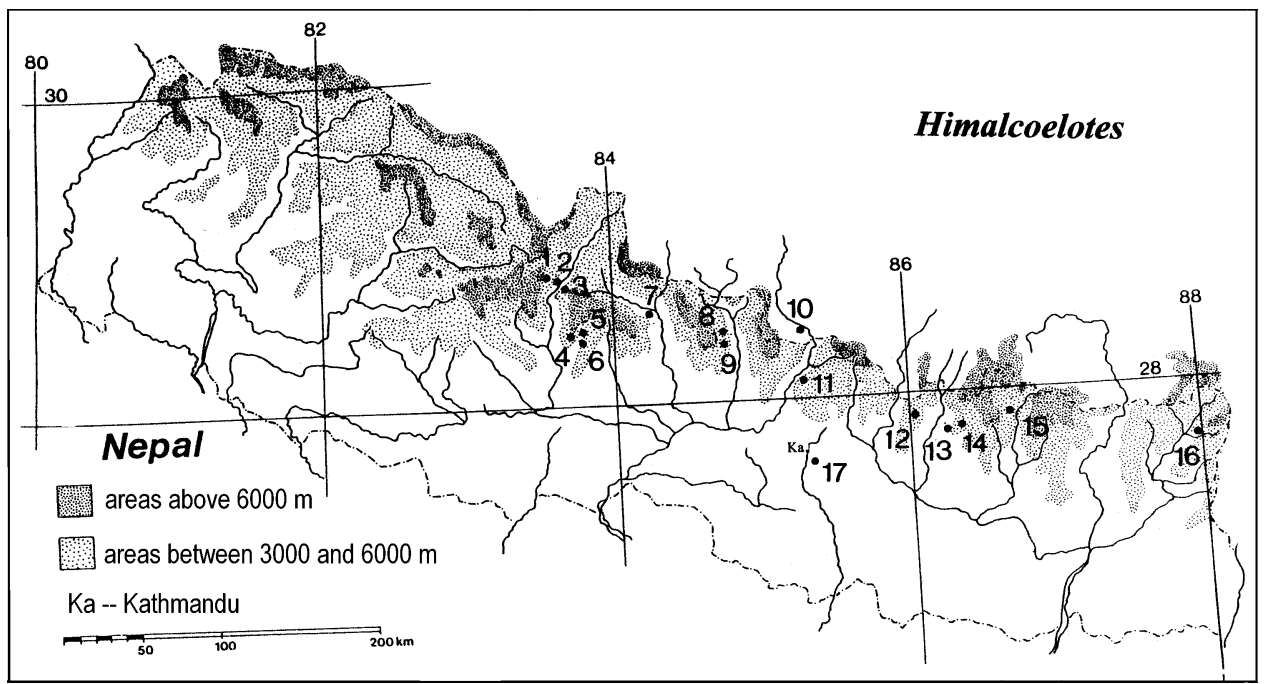

Map 10. Records of Himalcoelotes. The numbers represent: 1, upper Dambush Khola Valley above Tukche (H. aequoreus); 2, Tukche (H. aequoreus, H. martensi); 3, Thaksang above Tukche (H. aequoreus); 4, Ghorepani Pass (H. aequoreus, H. gyirongensis, H. martensi); 5, near Landrung and Kuldigar (H. gyirongensis); 6, Dobang (H. aequoreus); 7, above Bagarchap (H. pirum); 8, Rupina La (H. diatropos); 9, Dorondi Khola Valley down to above Barpak (H. diatropos); 10, Kyerong, south Tibet $(H$. gyirongensis); 11, Syngyang (H. diatropos); 12, Ting Sang La (H. bursarius); 13, Jiri (H. subsherpa); 14, Thodung (H. sherpa, H. subsherpa); 15, Taboche, Yaral (H. sherpa); 16, Tseram (H. sherpa); 17, Phulchoki Mt. (H. syntomos) (prepared by J. Martens).

ETYMOLOGY: The specific name refers to the smooth epigynum and less apparent atrium.

DiAgnosis: This new species is similar to $H$. primus but differs in the less apparent atrium, the small copulatory ducts, and the strongly convoluted spermathecae of females (figs. 242, 243), and by the strongly elongated cymbial furrow and the different shape of the RTA of males (figs. 244, 245)

DESCRIPTION: Female (holotype): Total length 8.50. Carapace 3.90 long, 2.36 wide. Abdomen 4.60 long, 3.20 wide. Eye sizes and interdistances: AME 0.16, ALE 0.19, PME 0.18, PLE 0.20; AME-AME 0.10, AME-ALE 0.08, PME-PME 0.15, PME-PLE 0.22, AME-PME 0.19. Clypeus height 0.28. Leg measurements: I, $10.2(2.84,3.60,2.40$, 1.40); II, $9.28(2.62,3.14,2.26,1.26)$; III, 8.44 (2.34, 2.70, 2.20, 1.20); IV, 11.0 (2.94, $3.58,3.00,1.44)$. Epigynal teeth long, situated anteriorly, with bases close together; atrium small; copulatory ducts short, not visible in dorsal view; spermathecal heads small, situated anteriorly; spermathecae broad, convoluted (figs. 242, 243).
Male (paratype): Total length 8.60. Carapace 4.40 long, 3.00 wide. Abdomen 4.20 long, 2.80 wide. Eye sizes and interdistances: AME 0.23, ALE 0.23, PME 0.20, PLE 0.21; AME-AME 0.07, AME-ALE 0.04, PMEPME 0.17, PME-PLE 0.24, AME-PME 0.17. Clypeus height 0.25. Leg measurements: I, 14.6 (3.78, 4.60, 3.84, 2.34); II, 13.5 (3.52, 4.20, 3.60, 2.14); III, 11.8 (3.20, 3.60, 3.24, 1.72); IV, 15.2 (4.02, 4.60, 4.52, 2.02). Palpal patellar apophysis moderately long; RTA long, occupies most of tibia, with distal end slightly extended; lateral tibial apophysis small; cymbial furrow long, occupies most of cymbial length, with dorsal edge strongly concave; conductor long, with slender apex; conductor dorsal apophysis broad, short; conductor lamella large; embolus posterior in origin; median apophysis small, modified (figs. 244, 245).

Other Material Examined: NEPAL: Parbat Dist., Gorapani (= Ghorepani) pass, 2850-2900 m, December 10-14, 1969, 1 female (J. Martens, SMF, no.31); Mustang Dist., Dambush Valley above Tukche, alpine dwarf bushes, 3770-4100 m, October 7-13, 


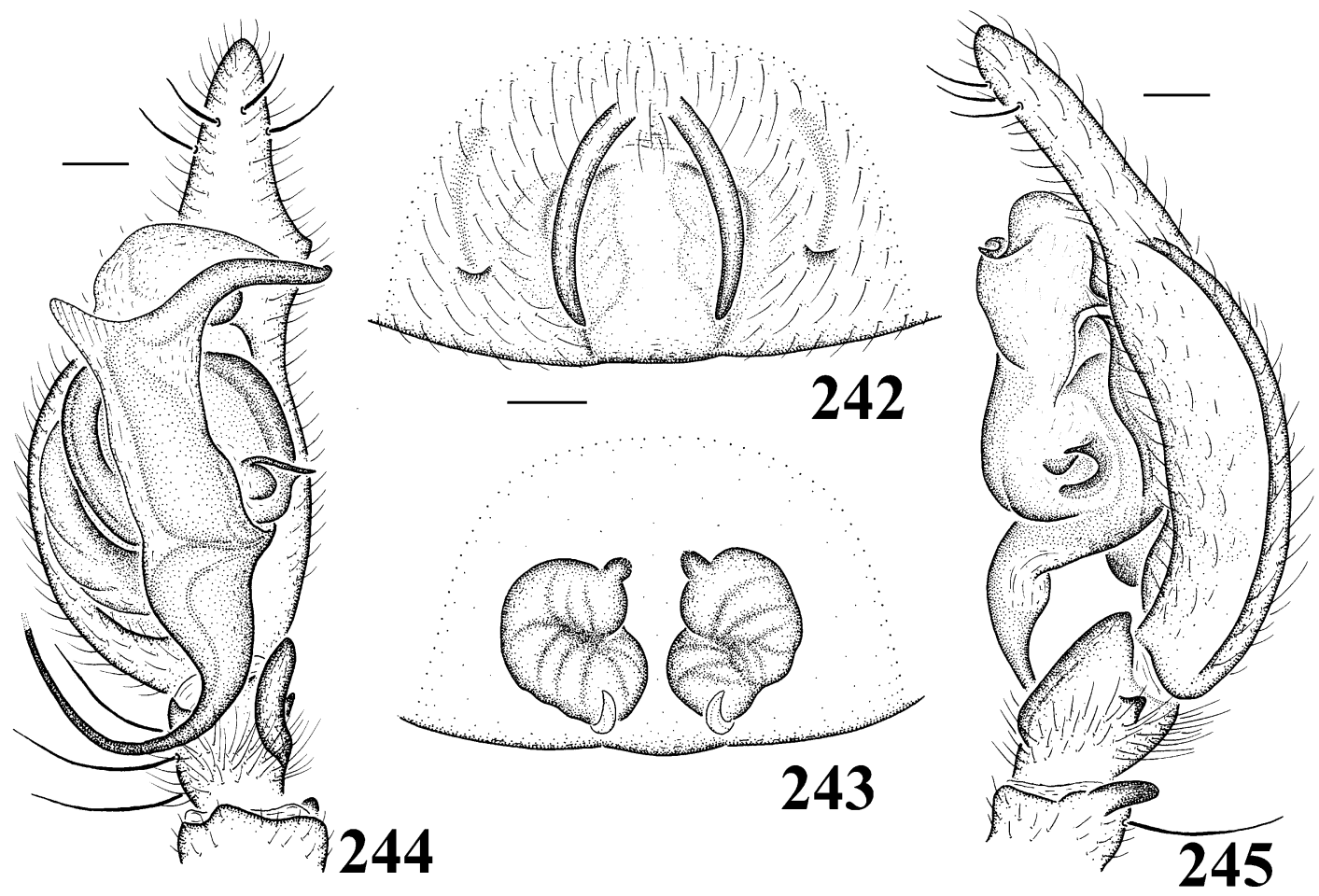

Figs. 242-245. Himalcoelotes aequoreus, new species. 242. Female epigynum. 243. Female vulva. 244. Male palp, ventral view. 245. Male palp, retrolateral view.

1969, 2 females (J. Martens, SMF, no. 21); Kaski Dist, near Dobang, ca. 2500 m, bamboo forest, October 23, 1985, 1 female (J. Coddington, USNM).

Distribution: Nepal.

\section{Himalcoelotes brignolii, new species} Figures 246, 247

Coelotes baronii Brignoli, 1978a: 42, figs. 19, 20, female only (female paratype from Dorjula, Bhutan, in NHMB, examined). Mismatched.
TYPE: Female holotype (female paratype of Coelotes baronii Brignoli, 1978) from Dorjula, 3100 m, Bhutan (June 6, 1972), in NHMB (2302b), examined.

ETYMOLOGY: The specific name is a patronym in honor of the late Prof. P. M. Brignoli.

Diagnosis: The females are similar to those of $H$. diatropos but differ in the less apparent atrium and the different shape of spermathecae (figs. 246, 247).


Figs. 246, 247. Himalcoelotes brignolii, new species, female. 246. Epigynum. 247. Vulva. 




Figs. 248, 249. Himalcoelotes bursarius, new species, female. 248. Epigynum. 249. Vulva.

DESCRIPTION: The female was described by Brignoli (1978a, as female paratype of Coelotes baronii). Epigynum with atrium shallow, less apparent; epigynal teeth long, situated medially on epigynum, with posterior ends almost reach epigastric furrow; spermathecal heads small, slender, situated anteriorly; spermathecae broad, convoluted (figs. 246, 247).

Male: Unknown.

OTHER MATERIAL EXAMINED: None.

Distribution: Bhutan.
Himalcoelotes bursarius, new species

Figures 248, 249

TYPE: Female holotype and 8 female paratypes from Sindhu Palchok Dist., pass Ting Sang La above Barabise, mixed forest, 3400 m, Nepal (April 13-15, 1973; J. Martens), in SMF (no.42), examined.

ETYMOLOGY: The specific name refers to the presence of apparent copulatory ducts in dorsal view.

Diagnosis: The females are similar to
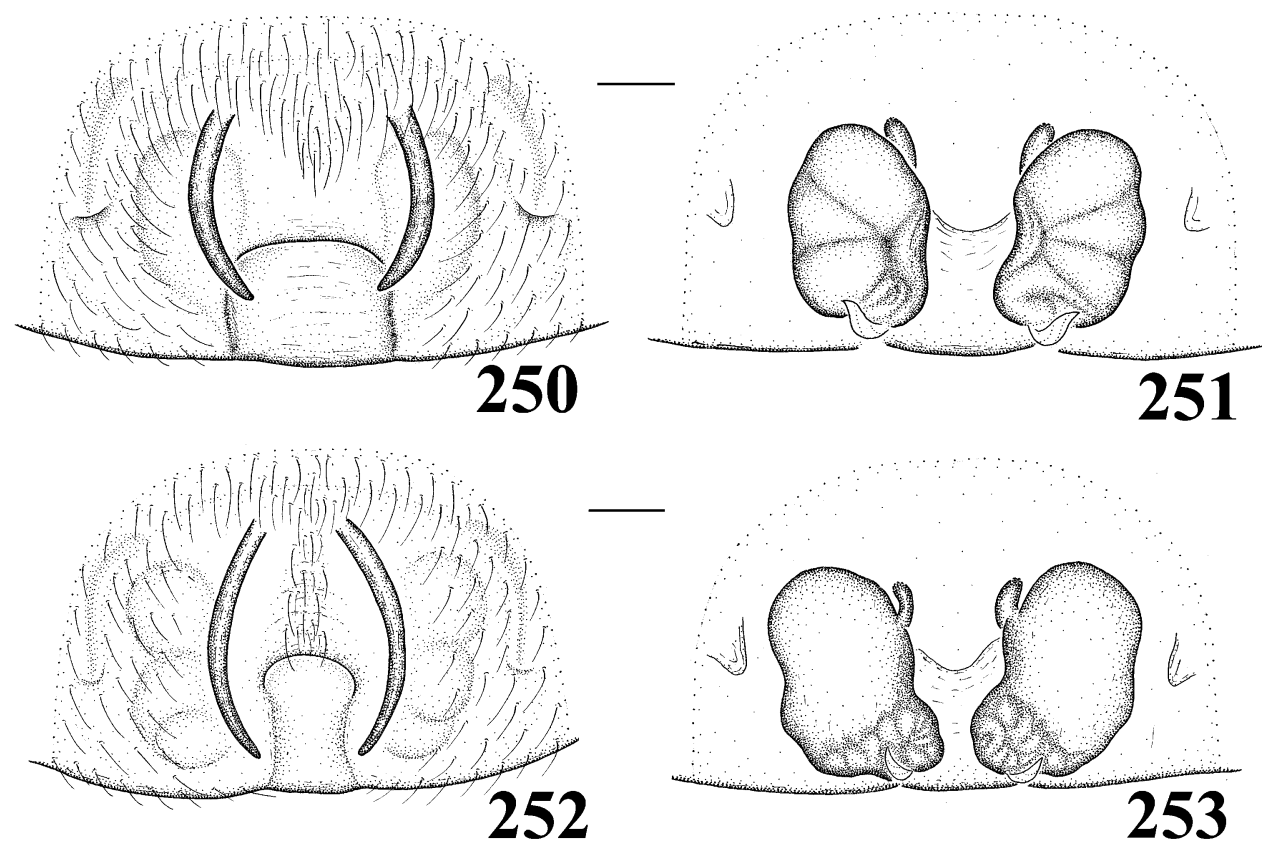

Figs. 250-253. Himalcoelotes diatropos, new species, female. 250. Epigynum (holotype). 251. Vulva (holotype). 252. Epigynum (showing variation). 253. Vulva (showing variation). 

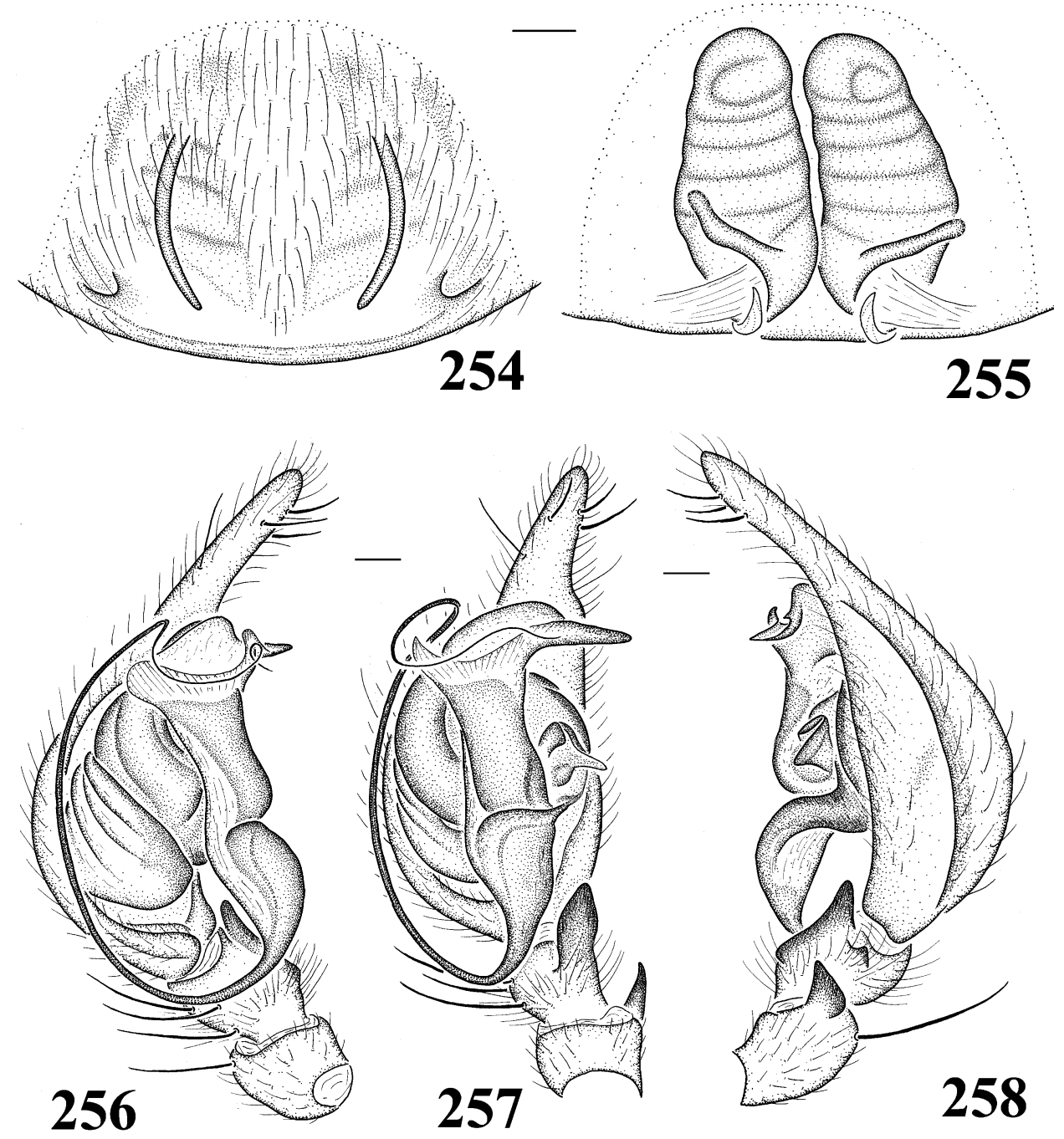

Figs. 254-258. Himalcoelotes gyirongensis (Hu and Li). 254. Female epigynum. 255. Female vulva. 256. Male palp, prolateral view. 257. Male palp, ventral view. 258. Male palp, retrolateral view.

those of $H$. sherpa and $H$. subsherpa but can be distinguished by the different shape of the anterior atrial margin and the broad copulatory ducts (figs. 248, 249).

DESCRIPTION: Female (holotype): Total length 10.5. Carapace 5.30 long, 3.46 wide. Abdomen 5.30 long, 3.60 wide. Eye sizes and interdistances: AME 0.21, ALE 0.24, PME 0.20, PLE 0.23; AME-AME 0.12, AME-ALE 0.11, PME-PME 0.20, PME-PLE 0.33, AME-PME 0.23. Clypeus height 0.35. Leg measurements: I, 14.4 (3.92, 4.94, 3.60,
1.96); II, 13.4 (3.62, 4.56, 3.40, 1.80); III, 12.1 (3.40, 3.80, 3.20, 1.66); IV, 15.0 (4.00, $4.82,4.26,1.92)$. Epigynal teeth long; anteriorly situated, widely separated; atrium with apparent anterior atrial margin; copulatory ducts broad, apparent in dorsal view; spermathecal heads long, slender; spermathecae small, rounded, not convoluted (figs. 248, 249).

Male: Unknown.

Other Material Examined: None.

DistRibution: Nepal. 

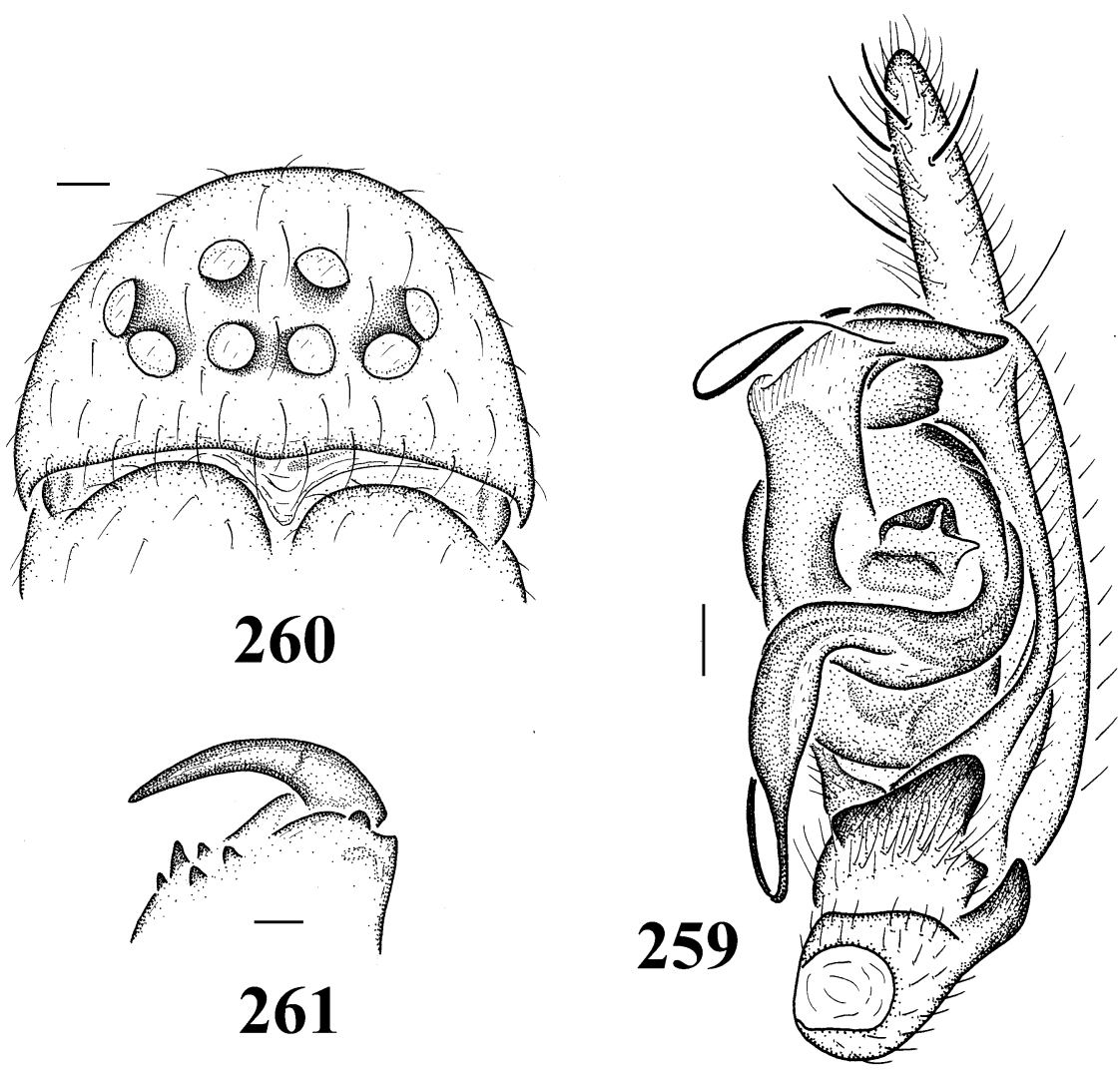

Figs. 259-261. Himalcoelotes gyirongensis (Hu and Li). 259. Male palp, retrolateral view, showing conductor dorsal apophysis. 260. Carapace, front view. 261. Chelicera, ventral view.

Himalcoelotes diatropos, new species Figures 250-253

TYPE: Female holotype and 1 female paratype from Gosainkund, Syng Gyangm, 3200 $\mathrm{m}$, mixed rhododendron-coniferous forest, Rasuwa Dist., Nepal (April 26, 1973; J. Martens), in SMF (no.41), examined.

ETYMOLOGY: The specific name derives from the variation of atrium size of female epigynum.

DiAGNOSIS: The females are similar to those of $H$. brignolii but can be distinguished by the presence of a large atrium and the broad spermathecae (figs. 250-253).

DESCRIPTION: Female (holotype): Total length 11.2. Carapace 4.80 long, 3.40 wide. Abdomen 6.40 long, 5.00 wide. Eye sizes and interdistances: AME 0.21, ALE 0.25, PME 0.24, PLE 0.23; AME-AME 0.10, AME-ALE 0.11, PME-PME 0.21, PME-PLE
0.30, AME-PME 0.27. Clypeus height 0.31. Leg measurements: I, 12.4 (3.60, 4.24, 2.84, 1.72); II, $11.2(3.20,3.80,2.66,1.58)$; III, $10.1(2.72,3.30,2.68,1.42)$; IV, 13.1 (3.58, $4.28,3.62,1.66)$. Epigynal teeth long, anteriorly situated, widely separated; atrium with anterior atrial margin (one specimen with much small atrium, fig. 252); copulatory ducts broad, originating mesally; spermathecal heads small; spermathecae broad, convoluted (figs. 250-253).

Male: Unknown.

Other Material Examined: NEPAL: Gorkha Dist., Darondi Khola to above Barpak, 3600-3450 m, August 10, 1983, 1 female (J. Martens and W. Schawaller, SMF, no. 242); Gorkha Dist., NW pass Rupina La, Tabruk Kharka, 4000 m, August 7-8, 1983 (J. Martens and W. Schawaller, SMF, no. 237). 

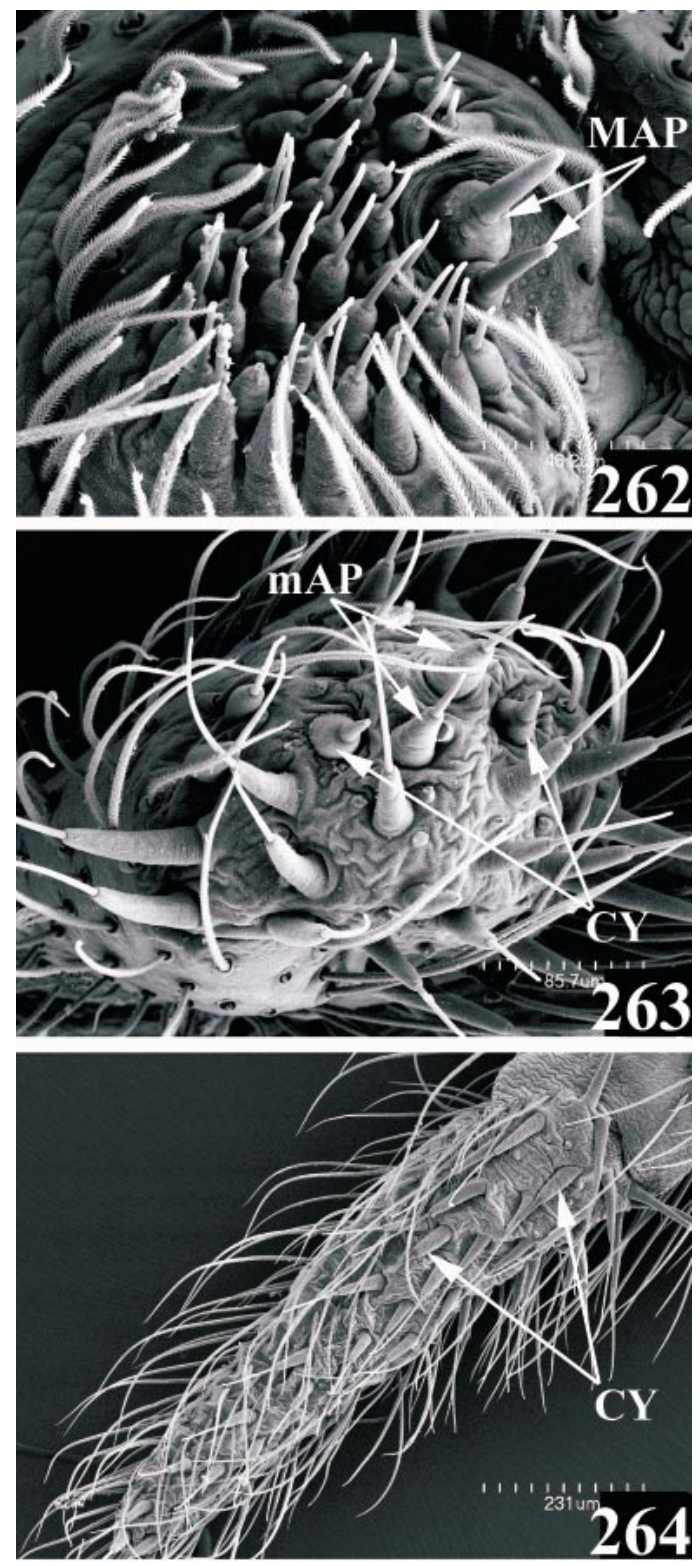

Figs. 262-264. Himalcoelotes gyirongensis (Hu and Li), female spinnerets, ventral view, left. 262. ALS. 263. PMS. 264. PLS. Abbreviations are spelled out on p. 6.

DistRIBUTION: Nepal.

Himalcoelotes gyirongensis ( $\mathrm{Hu}$ and $\mathrm{Li})$, new combination

Figures 254-264

Coelotes gyirongensis $\mathrm{Hu}$ and $\mathrm{Li}, 1987$ : 279, figs. 19.1-19.5 (female holotype, deposited in Shan- dong University, Ji-Nan, China, not examined). - Song et al., 1999: 375, figs. 217U, 217V.

Diagnosis: Resembles $H$. martensi in having large, anteriorly extended spermathecae, but can be easily distinguished by the widely separated, posteriorly situated atria and the laterally originating, posteriorly situated copulatory ducts of females (figs. 254, 255), and by the short, ventrally curved patellar apophysis, the elongated cymbial furrow, and the posteriorly origin of the embolus in males (figs. 256-259).

DESCRIPTION: Epigynal teeth long, slender, anteriorly situated, widely separated; atria small, situated posteriorly near epigastric furrow, widely separated; copulatory ducts orginated laterally, widely separated; spermathecal heads long, slender, situated posteriorly to ventral spermathecae, extended laterally; spermathecae strongly elongated anteriorly, strongly convoluted, close together (figs. 254, 255).

Female spinnerets with apex of ALS with 2 major ampullate gland spigots (MAP), 37 piriform gland spigots; PMS with 2 minor ampullate gland spigots (mAP), about 30 aciniform gland spigots, 2 cylindrical gland spigots (CY); PLS with about 27 aciniform gland spigots and 2 cylindrical gland spigots (figs. 262-264).

Male (Parbat Dist. specimen): Total length 10.5. Carapace 4.48 long, 3.16 wide. Abdomen 6.00 long, 2.80 wide. Eye sizes and interdistances: AME 0.23, ALE 0.21, PME 0.20, PLE 0.21; AME-AME 0.10, AMEALE 0.07, PME-PME 0.19, PME-PLE 0.25, AME-PME 0.20. Leg measurements: I, 17.7 (4.52, 6.00, 4.80, 2.40); II, 15.7 (4.32, 5.08, 4.16, 2.12); III, 12.8 (3.76, 4.24, 3.80, 1.76); IV, 17.4 (4.52, 5.60, 5.20, 2.12). Palpal patellar apophysis short, broad, with apex slightly curved ventrally; RTA long, occupies most of tibia, with distal end extended anteriorly; lateral tibial apophysis apparent; cymbial furrow long, occupies most of cymbial length, with dorsal edge strongly concave; conductor relatively long, slender; conductor dorsal apophysis broad (fig. 259); conductor lamella well developed; embolus posterior in origin, long; median apophysis small, modified (figs. 256-259).

Notes: The original descriptions and illus- 


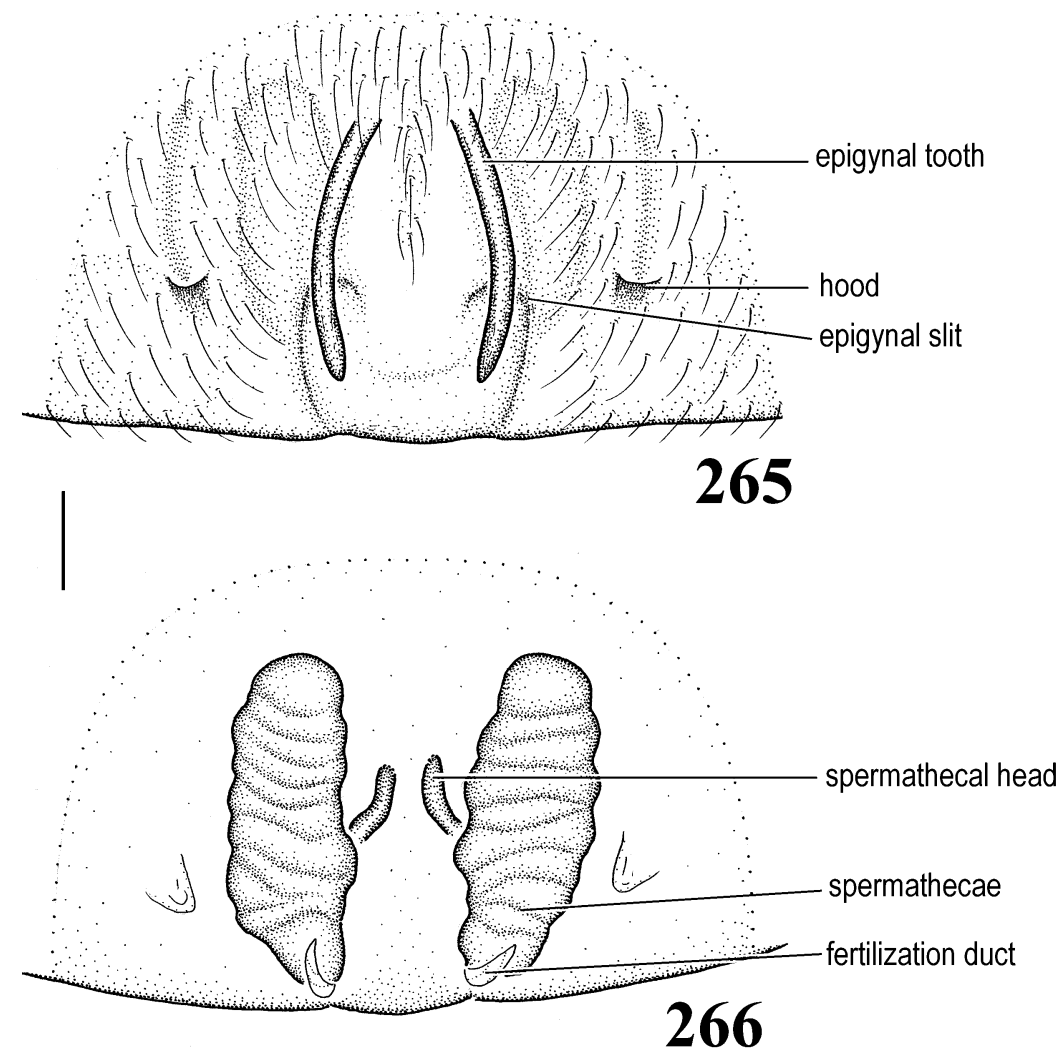

Figs. 265, 266. Himalcoelotes martensi, new species, female. 265. Epigynum. 266. Vulva.

trations of this species ( $\mathrm{Hu}$ and $\mathrm{Li}, 1987$ ) were not detailed enough for species identification, especially regarding the morphology of atria, copulatory ducts, and spermathecal heads. The types are not available. Specimens examined here are considered $H$. gyirongensis because of their similarity in spermathecal morphology. Comparison of this material with the types of $H$. gyirongensis is needed to confirm this identification.

Material Examined: NEPAL: Parbat Dist., near Ghorepani (= Gorapani), $2850 \mathrm{~m}$, October 27, 1985, 1 male and 1 female (J. Coddington, USNM); Kaski Dist., BhichukLandrung Trail, streamside, ca. 1650 m, October 20, 1985, 1 male (J. Coddington, USNM); Kaski Dist., rhododendron-bamboo forest near Khuldighar, 2400 m, October 22, 1985, 1 male (J. Coddington, USNM).

Distribution: Tibet, Nepal.
Himalcoelotes martensi, new species Figures 265-277

TYPE: Female holotype and 1 male paratype from Dambush Khola near Tukche, pitfall traps, 2900 m, Kaski Dist., Nepal (October 17-November 28, 1969; J. Martens), in SMF (no. 20), examined.

ETYMology: The specific name is a patronym in honor of the collector of the specimens, Prof. J. Martens.

DiAGNOSIS: This new species can be easily recognized by the reduced atrium, the position of spermathecal heads, and the large, anteriorly extended spermathecae of females (figs. 265, 266), and by the broad conductor and the prolateral origin of the embolus of males (figs. 267, 269).

DesCRIPTION: Female (holotype): Total length 10.0. Carapace 4.60 long, 2.86 wide. Abdomen 5.40 long, 3.40 wide. Eye sizes 


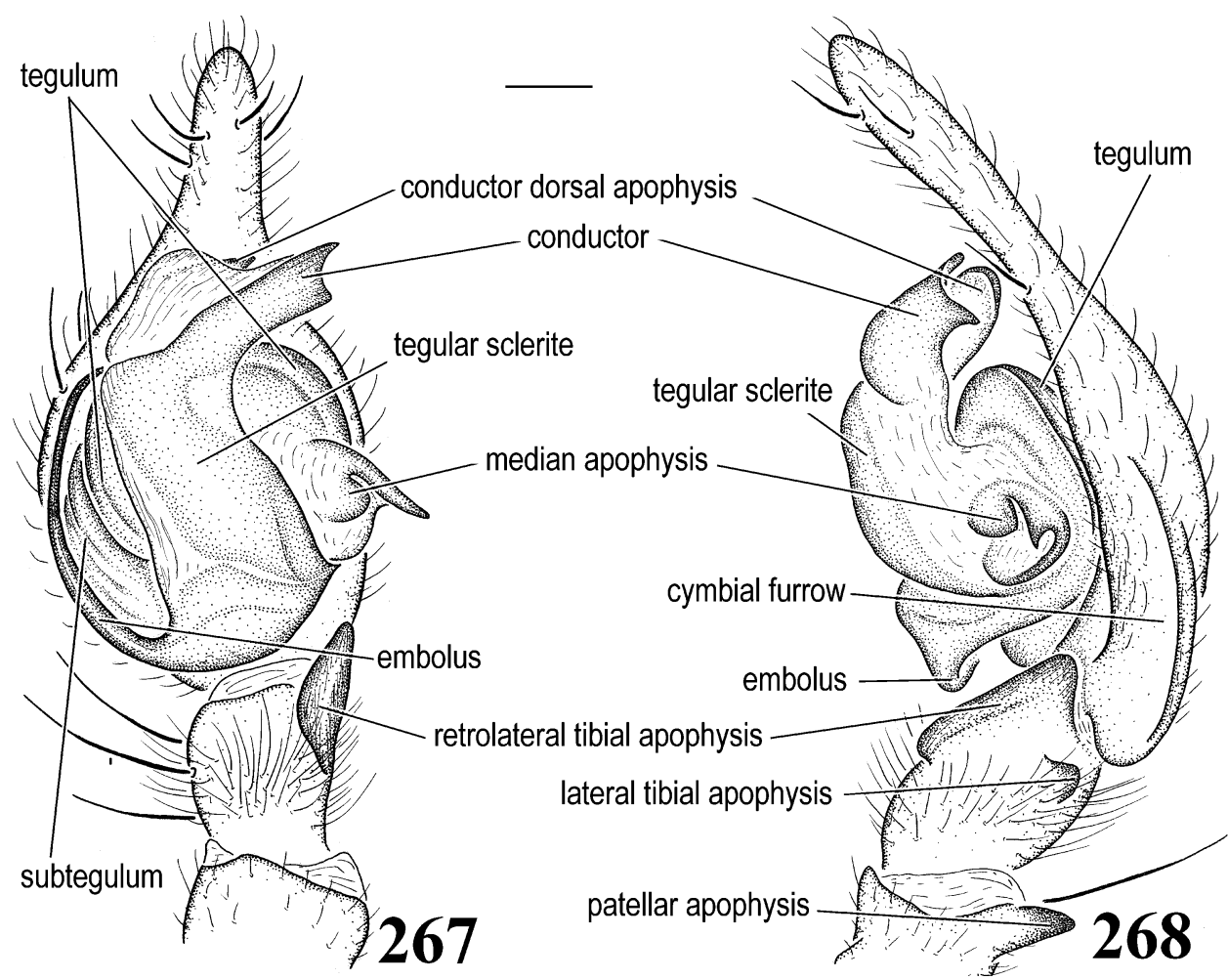

Figs. 267, 268. Himalcoelotes martensi, new species, male palp. 267. Ventral view. 268. Retrolateral view.

and interdistances: AME 0.21, ALE 0.23, PME 0.20, PLE 0.21; AME-AME 0.12, AME-ALE 0.09, PME-PME 0.20, PME-PLE 0.24, AME-PME 0.20. Clypeus height 0.26 (fig. 29). Leg measurements: I, 11.7 (3.20, $4.00,2.82,1.72)$; II, $10.7(2.98,3.58,2.60$, 1.56); III, 9.40 (2.64, 3.08, 2.46, 1.22); IV, 12.5 (3.44, 4.12, 3.40, 1.50). Clypeal height slightly longer than AME diameter; chilum divided, hairless (fig. 270). Chelicerae with three promarginal and two retromarginal teeth (fig. 271). Labial about as long as wide. Length of female 1st leg patella + tibia shorter than carapace length (4.0/4.6). Epigynum with long epigynal teeth, anteriorly situated close together; atrium less apparent; hoods more or less apparent; copulatory ducts short, not visible in dorsal view; spermathecal heads long, slender, situated mesally; spermathecae convoluted, strongly elongated anteriorly, with indistinct stalks and bases (figs. 265, 266).
Male (paratype): Total length 7.90. Carapace 4.10 long, 2.72 wide. Abdomen 3.80 long, 3.60 wide. Eye sizes and interdistances: AME 0.20, ALE 0.19, PME 0.18, PLE 0.20; AME-AME 0.09, AME-ALE 0.06, PMEPME 0.11, PME-PLE 0.20, AME-PME 0.17. Clypeus height 0.28. Leg measurements: I, 14.0 (3.68, 4.64, 3.66, 2.02); II, 12.9 (3.46, 4.08, 3.48, 1.92); III, 11.9 (3.14, 3.60, 3.40, 1.72); IV, 14.6 (3.86, 4.40, 4.34, 2.02). Male palp with moderately long patellar apophysis; RTA slightly longer than half tibial length, with distal end not strongly extended; lateral tibial apophysis large; cymbial furrow short, not strongly elongated; conductor broad, with apex slightly bifid; conductor dorsal apophysis broad; conductor lamella not well developed; embolus prolateral in origin; median apophysis small, rounded, modified with deep notch and sharp extension (figs. 267, 269).

Tarsal trichobothria with large hood trans- 

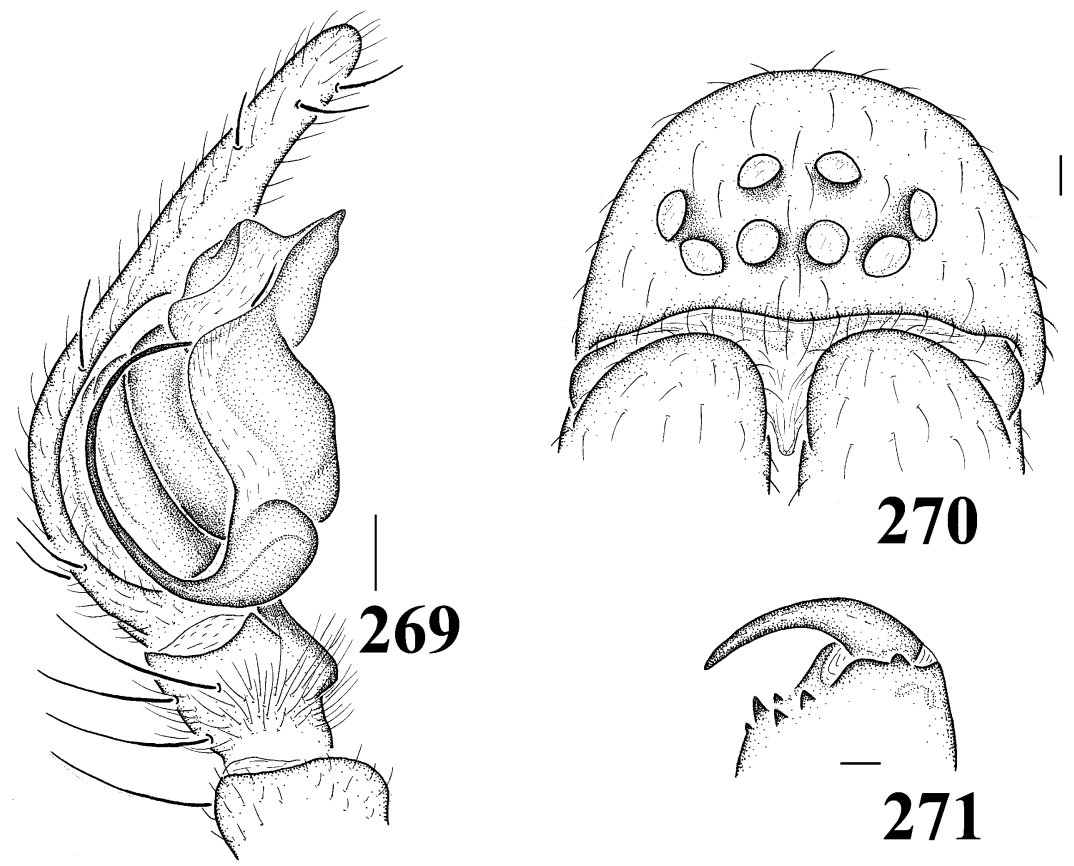

Figs. 269-271. Himalcoelotes martensi, new species. 269. Male palp, prolateral view. 270. Carapace, front view. 271. Chelicera, ventral view.

versely striated, small hood smooth (fig. 272); tarsal organ small, rounded (fig. 273), situated on distal end of tarsus, anterior of distalmost trichobothrium (fig. 274, double arrows). Trachea not examined. Examined female spinnerets not in good condition, particular PLS. Apex of ALS with 2 major ampullate gland spigots (MAP), 24 piriform gland spigots; PMS with at least 8-10 aciniform gland spigots, 2 minor ampullate gland spigots (mAP), and 2 cylindrical gland spigots (CY); PLE with at least 10 aciniform gland spigots, only 1 cylindrical gland spigot seen on observed specimen (figs. 275-277).

Other Material Examined: NEPAL: Kaski Dist., Dambush Khola near Tukche, pitfall traps, $2900 \mathrm{~m}$, October 17-November 28, 1969, 1 male (J. Martens, SMF, no. 20); Mustang Dist., forest clearing, Thaksang above Tukche, 3100-3300 m, February 27March 11, 1974, 1 female (J. Martens, SMF, no. 25); Parbat Dist., Gorapani Pass (=Ghorepani Pass), 2850-2900 m, December 1014, 1969, 1 male (J. Martens, SMF, no. 31).

Distribution: Nepal.
Himalcoelotes pirum, new species

Figures 278-281

TYPE: Female holotype (no. 139) from Marsyandi Valley, above Bagarchap, AcerQuercus mixed forest, Manang Dist., Nepal (April 13-14, 1980; J. Martens and Ausobsky), and male paratype (no. 138) from Marsyandi Valley, above Bagarchap, Acer-Quercus mixed forest, Manang Dist., Nepal (April 12-13, 1980; J. Martens and Ausobsky), in SMF, examined.

ETYMOLOGY: The specific name refers to the pear-shaped spermathecae.

Diagnosis: This species is similar to $H$. aequoreus but can be distinguished by the large atrium and the pear-shaped, less convoluted spermathecae of females (figs. 278, 279 ), and by the short cymbial furrow of males (figs. 280, 281).

DESCRIPTION: Female (holotype): Total length 10.5. Carapace 4.90 long, 3.00 wide. Abdomen 5.60 long, 4.00 wide. Eye sizes and interdistances: AME 0.16, ALE 0.21, PME 0.20, PLE 0.20; AME-AME 0.12, 

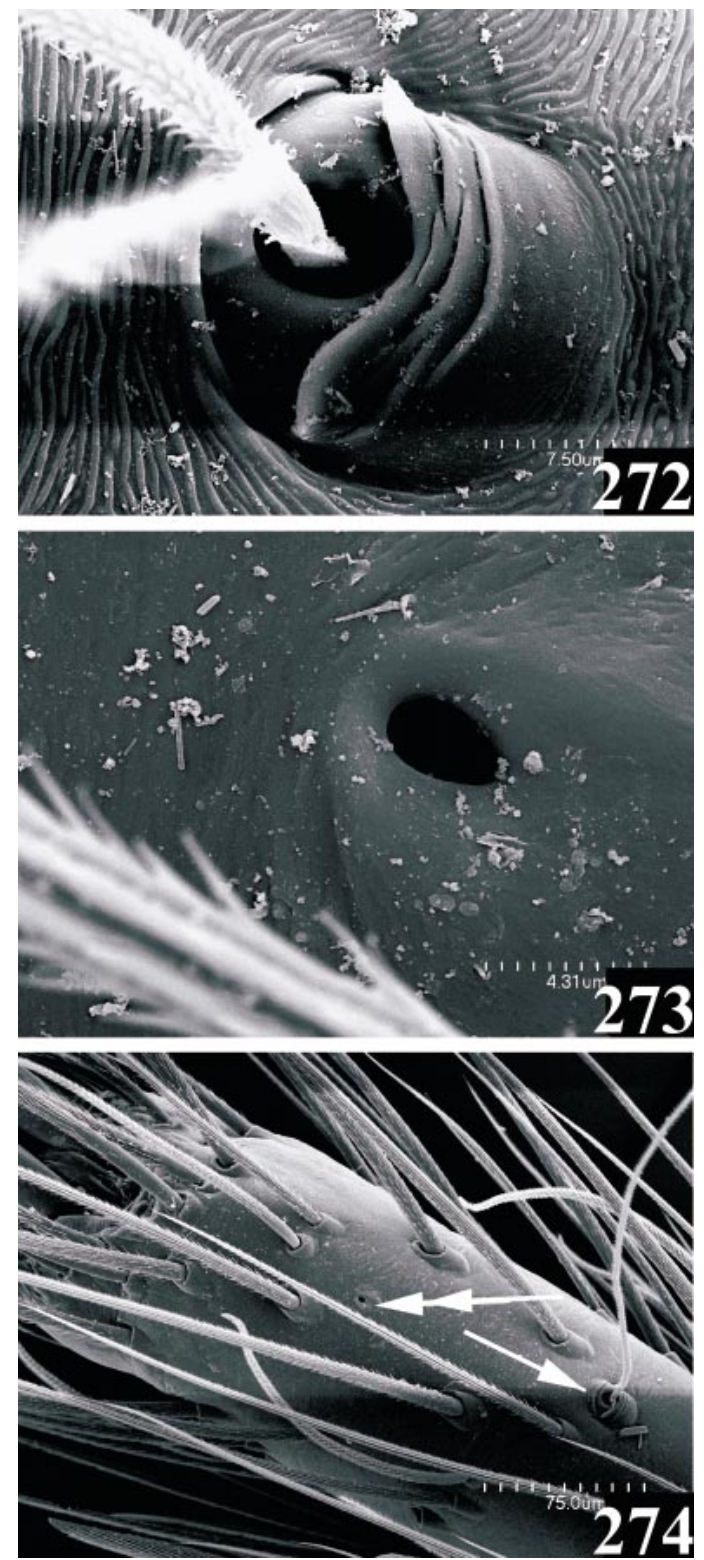

Figs. 272-274. Himalcoelotes martensi, new species. 272. Trichobothrium. 273. Tarsal organ. 274. Tarsus, showing position of tarsal organ (double arrows) and distalmost trichobothrium (single arrow).

AME-ALE 0.12, PME-PME 0.18, PME-PLE 0.25, AME-PME 0.19. Clypeus height 0.30. Leg measurements: I, 12.0 (3.36, 4.16, 2.76, $1.70)$; II, $10.7(3.06,3.60,2.50,1.58)$; III, $9.70(2.78,3.02,2.48,1.42)$; IV, 12.9 (3.44, $4.20,3.50,1.72)$. Epigynal teeth long, ante-
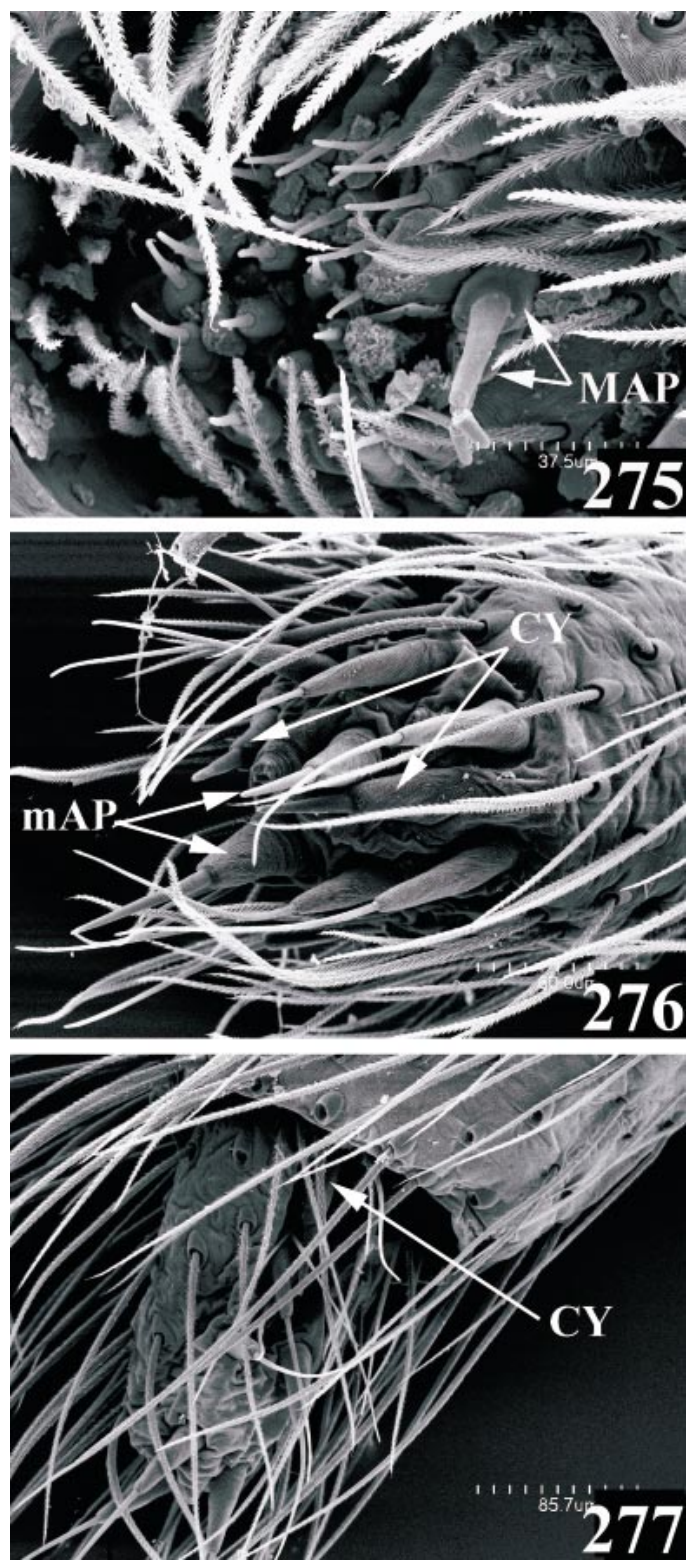

Figs. 275-277. Himalcoelotes martensi, new species, female spinnerets, ventral view, left. 275. ALS. 276. PMS. 277. PLS. Abbreviations are spelled out on p. 6 .

riorly situated, close together; atrium with apparent anterior atrial margin; copulatory ducts short, situated anteriorly between spermathecae, barely visible in dorsal view; spermathecal heads short, situated anteriorly; spermathecae broad, close together (figs. 278, 279). 

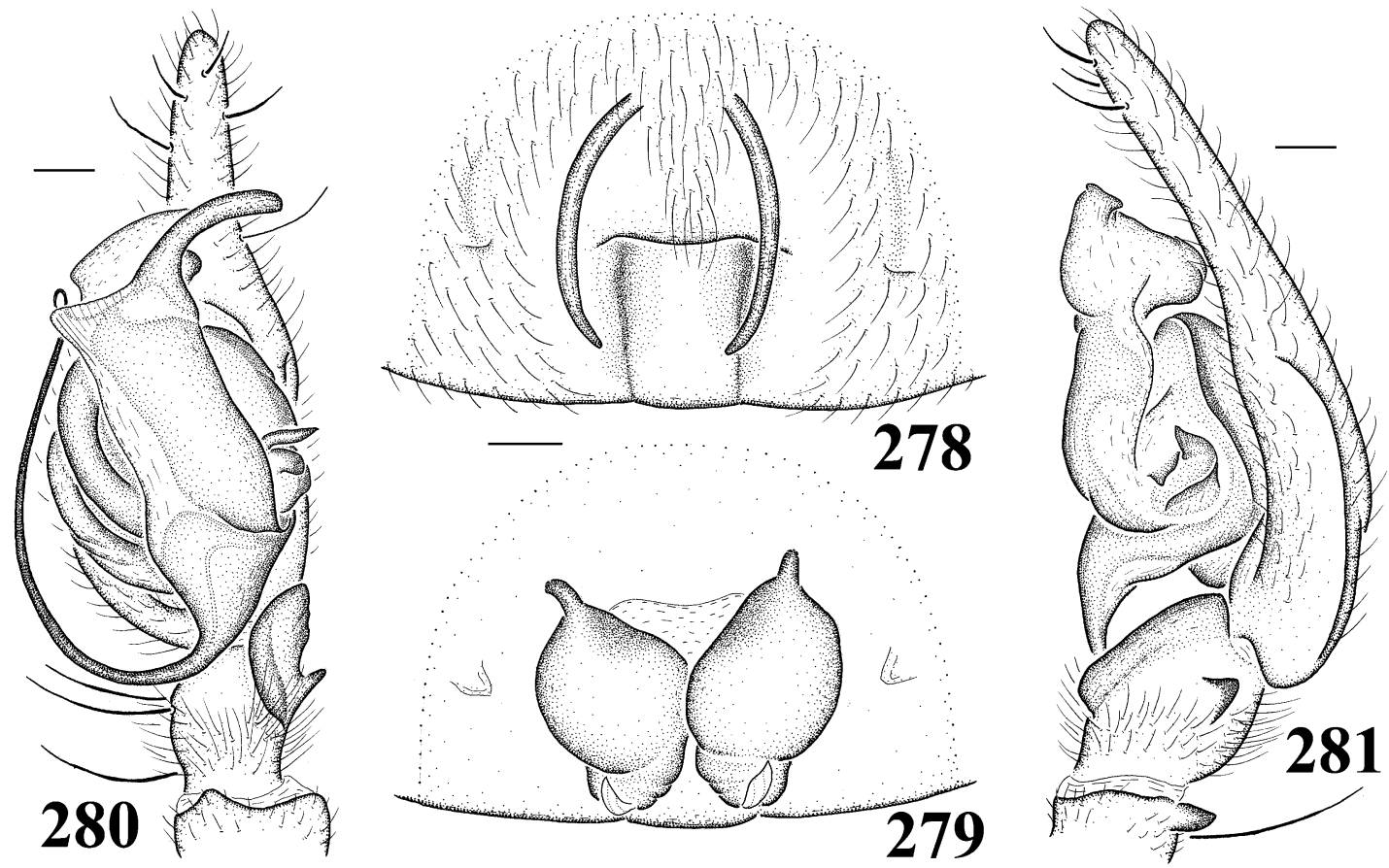

Figs. 278-281. Himalcoelotes pirum, new species. 278. Female epigynum. 279. Female vulva. 280. Male palp, ventral view. 281. Male palp, retrolateral view.

Male (paratype): Total length 9.20. Carapace 5.00 long, 3.20 wide. Abdomen 4.20 long, 3.00 wide. Eye sizes and interdistances: AME 0.19, ALE 0.23, PME 0.20, PLE 0.23; AME-AME 0.10, AME-ALE 0.07, PMEPME 0.16, PME-PLE 0.20, AME-PME 0.19. Clypeus height 0.34. Leg measurements: I, 13.8 (3.62, 4.70, 3.40, 2.06); II, 12.7 (3.40, 4.20, 3.20, 1.92); III, 9.74 (2.60, 2.96, 2.60, 1.58); IV, 14.6 (3.82, 4.60, 4.16, 2.00). Palpal patellar apophysis small; RTA broad, occupies most tibial length, slightly extended anteriorly; lateral tibial apophysis large; cymbial furrow moderately long, with dorsal edge strongly concave; conductor broad; conductor dorsal apophysis short, broad; conductor lamella moderately developed; embolus basal in origin; median apophysis small, modified (figs. 280, 281).

Other Material Examined: NEPAL: Manang Dist., Marsyandi Valley, above Bagarchap, Acer-Quercus mixed forest, 2200 m, April 12-13, 1980, 3 males (J. Martens and Ausobsky, SMF, no. 138).

DisTRIBUTION: Nepal.

\section{Himalcoelotes sherpa (Brignoli), new combination Figures 282-289}

Coelotes sherpa Brignoli, 1976: 237, figs. 11, 12 (female holotype from high altitude yak pasture, Solukhumbu Dist., Nepal, and 1 female paratype from Yaral, Solukhumbu Dist., Nepal, in ZII, examined).

Diagnosis: This species can be recognized by the absence of anterior atrial margin, the small, rounded, nonconvoluted spermathecae, and the short copulatory ducts of females (figs. 282-287), and by the long, strong patellar apophysis, the slender, bificated conductor, and the prolaterally originating embolus of males (figs. 288, 289).

DESCRIPTION: Epigynum with epigynal teeth long, widely separated; anterior atrial margin less developed, with atria widely separated; copulatory ducts short, invisible in dorsal view; spermathecal heads long, slender, situated anteriorly; spermathecae small, rounded, not convoluted (figs. 282-287).

Male with examined specimen in poor so- 

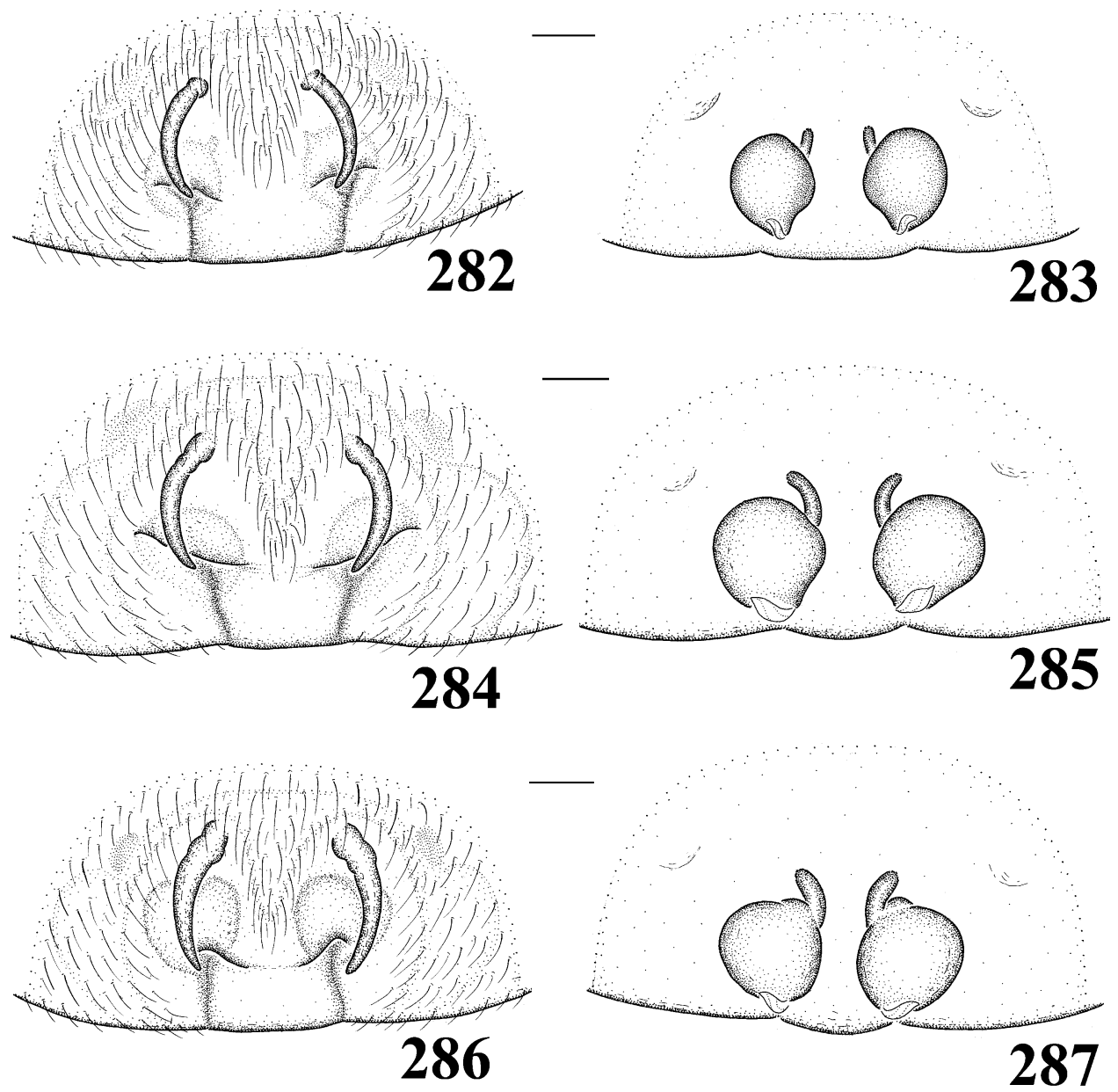

Figs. 282-287. Himalcoelotes sherpa, new species, female. 282, 284, 286. Epigynum. 283, 285, 287. Vulva.

matic condition. Palpal patellar apophysis relatively long, strong; RTA broad, with distal end extended anteriorly; lateral tibial apophysis large; cymbial furrow short; conductor slender, bifurcated; conductor dorsal apophysis short, broad; embolus prolateral in origin; median apophysis short, modified (figs. 288, 289).

MATERIAl EXAMINED: NEPAL: Ramechap Dist., Thodung near Those, $3200 \mathrm{~m}$, AbiesTsuga-rhododendron forest, September 3-7, 1970, 1 male and 1 female (J. Martens, SMF, no. 47); Taplejung Distr., Upper Simbua Khola Valley, near Tseram, 3250-3350 m, mature Abies-rhododendron forest, May 1015, 1988, 1 female (J. Martens and W. Schawaller, SMF, no. 361); Solukhumbu Dist., Ta- boche (= Taweche), yak pasture, dwarf bushes, $4550 \mathrm{~m}$, May 31, 1961, 1 female holotype (H. Janetschek, ZII, Np61-77); Solukhumbu Dist., Yaral (Pangpoche), caught at night with artificial light, 3900 m, May 25, 1961, 1 female paratype (H. Janetschek, ZII, Np61-206).

DisTRIBUTION: Nepal.

Himalcoelotes subsherpa, new species Figures 290-293

TYPES: Male holotype and female paratype from Thodung near Those, $3200 \mathrm{~m}$, Ramechap Dist., Nepal (April 3-9, 1973; J. Martens), in SMF (no. 48), examined.

ETYMOLOGY: The specific name refers to its similarity to the species $H$. sherpa. 


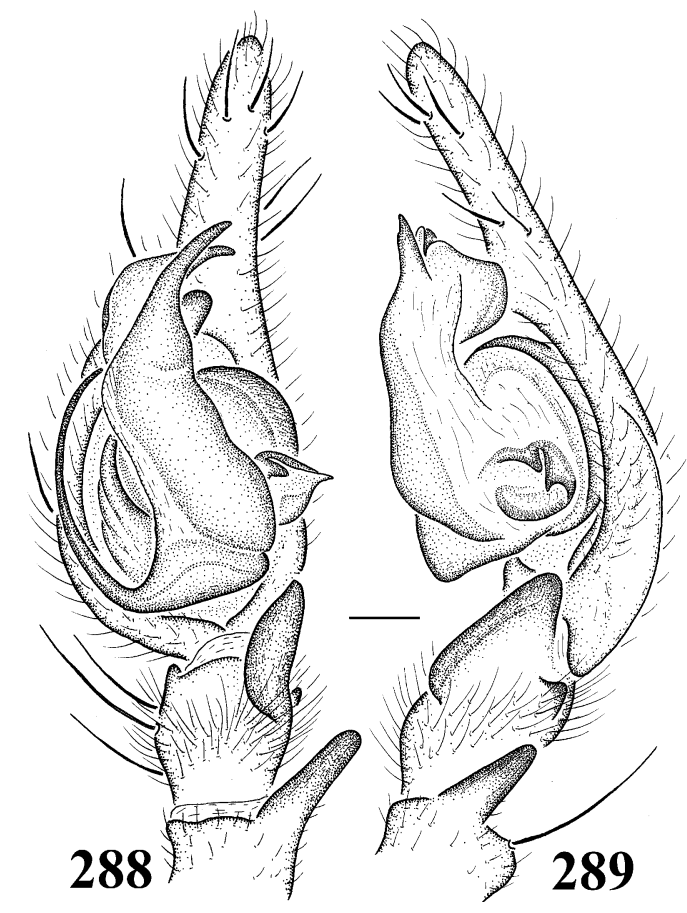

Figs. 288, 289. Himalcoelotes sherpa, new species, male palp. 288. Ventral view. 289. Retrolateral view.

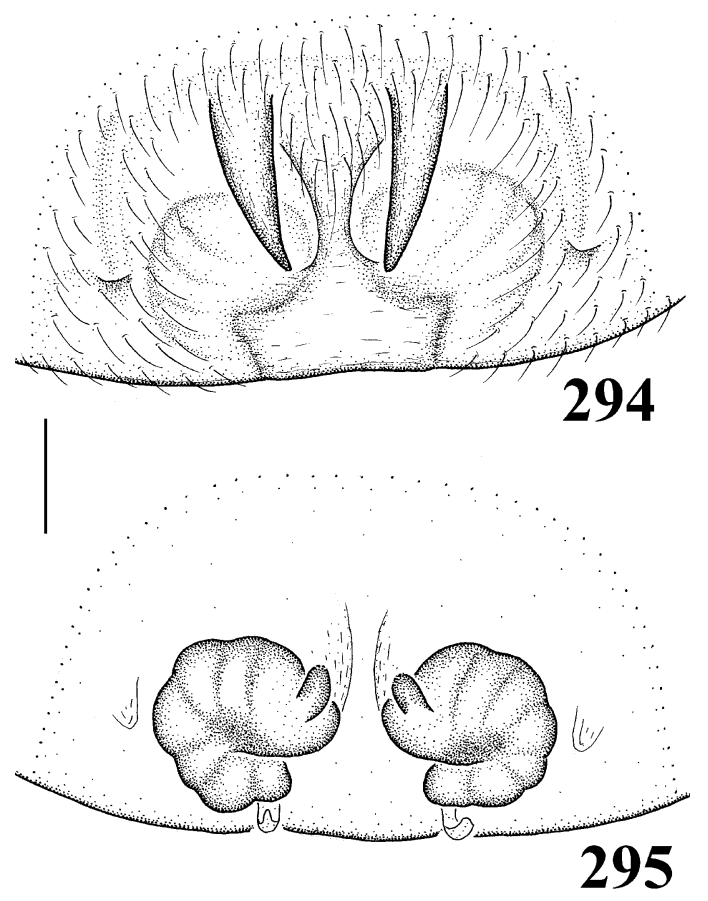

Figs. 294, 295. Himalcoelotes syntomos, new species, female. 294. Epigynum. 295. Vulva.
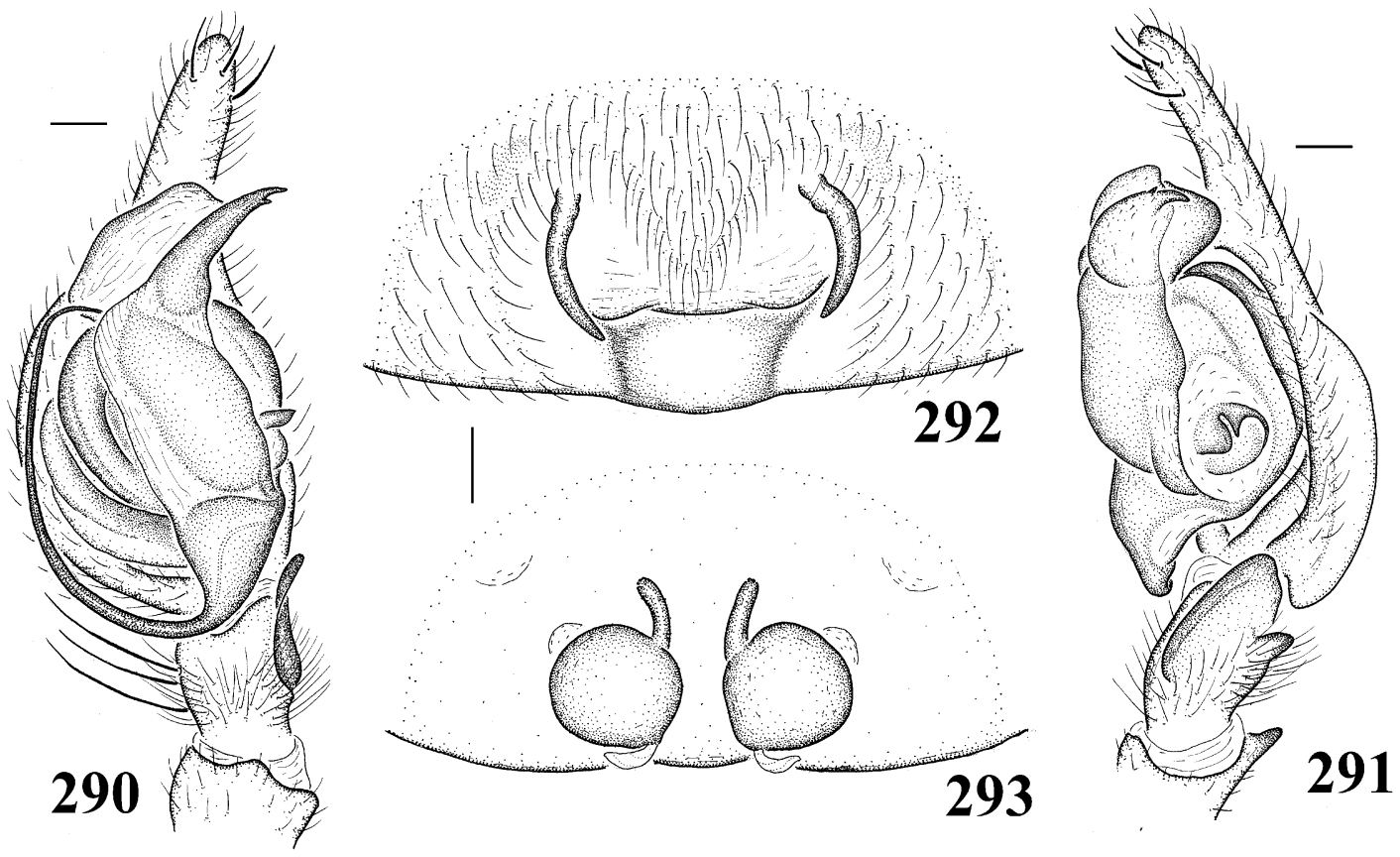

Figs. 290-293. Himalcoelotes subsherpa, new species. 290. Male palp, ventral view. 291. Male palp, retrolateral view. 292. Female epigynum. 293. Female vulva. 

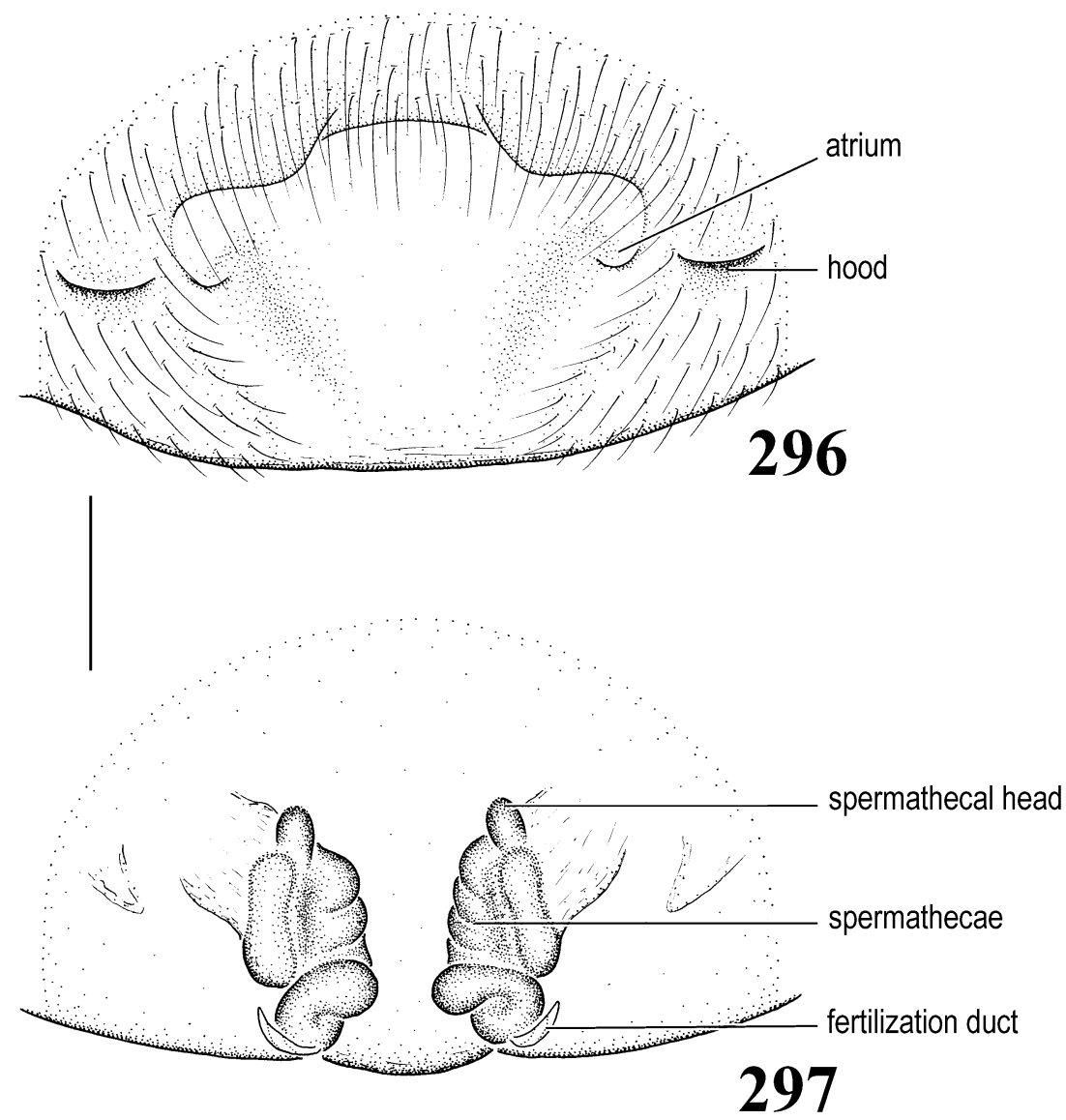

Figs. 296, 297. Leptocoelotes pseudoluniformis (Zhang, Peng and Kim), female. 296. Epigynum. 297. Vulva.

DiAGNOSIS: This new species is similar to $H$. sherpa but can be distinguished by the small patellar apophysis, the basally originated embolus, and the presence of a small tooth on distal conductor of males (figs. 290, 291), and by the differences of their atrium and the laterally originated copulatory ducts of females (figs. 292, 293).

DESCRIPTION: Male (holotype): Total length 8.80. Carapace 4.60 long, 3.20 wide. Abdomen 4.20 long, 2.80 wide. Eye sizes and interdistances: AME 0.23, ALE 0.22, PME 0.20, PLE 0.21; AME-AME 0.08, AME-ALE 0.06, PME-PME 0.13, PME-PLE 0.25, AME-PME 0.19. Clypeus height 0.26. Leg measurements: I, 15.0 (4.00, 5.00, 3.80, 2.20); II, 14.0 (3.78, 4.56, 3.66, 2.00); III, 12.7 (3.36, 4.00, 3.56, 1.78); IV, 15.8 (4.14, $5.10,4.58,2.00)$. Palpal patellar apophysis short, small; RTA broad, long, occupies most of tibia, with distal end slightly extended; lateral tibial apophysis large; cymbial furrow moderately long; conductor slender, with small tooth on distal end; conductor dorsal apophysis short, broad; conductor lamella moderately developed; embolus basal in origin; median apophysis small, modified (figs. 290, 291).

Female (paratype): Total length 11.0. Carapace 5.25 long, 3.75 wide. Abdomen 5.70 long, 4.20 wide. Eye sizes and interdistances: AME 0.26, ALE 0.28, PME 0.24, PLE 0.28; AME-AME 0.11, AME-ALE 0.10, PMEPME 0.22, PME-PLE 0.33, AME-PME 0.28. Clypeus height 0.40. Leg measurements: I, 15.3 (4.20, 5.20, 3.78, 2.12); II, 13.9 (3.84, 4.64, 3.46, 1.96); III, 12.3 (3.42, 3.98, 3.34, $1.60)$; IV, 15.8 (4.20, 5.18, 4.40, 2.02). Epi- 

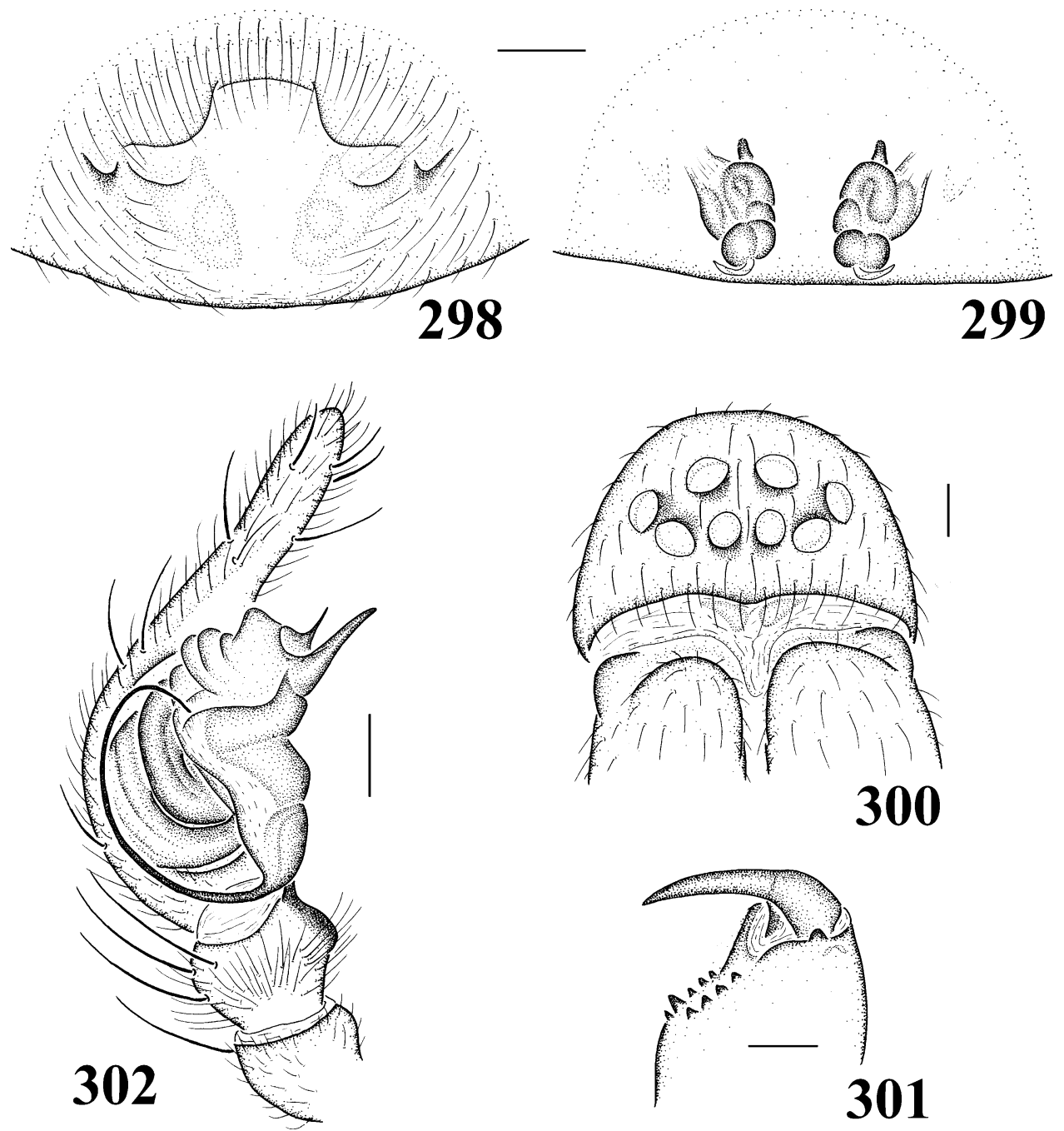

Figs. 298-302. Leptocoelotes pseudoluniformis (Zhang, Peng and Kim). 298. Female, epigynum. 299. Female, vulva. 300. Carapace, front view. 301. Chelicera, ventral view. 302. Male palp, prolateral view.

gynal teeth strong, anteriorly situated, widely separated; atrium with more or less apparent anterior atrial margin; copulatory ducts short, situated laterally, barely visible in dorsal view; spermathecal heads long, slender, situated anteriorly; spermathecae small, rounded, not convoluted (figs. 292, 293).

Other Material Examined: NEPAL: Dolakha Dist., Jiri Valley with Hanumante Mt., 2900 m, March 28-29, 1973, 1 female (J. Martens, SMF, no. 46).
DisTRIBUTION: Nepal.

Himalcoelotes syntomos, new species Figures 294, 295

TYPE: Female holotype from Kathmandu Valley, Phulchoki Mt., 2900 m, oak forest, Lalitpur Dist., Nepal (January 25-30, 1970; J. Martens), in SMF (no. 34), examined.

ETYMOLOGY: The specific name derives from the relatively short epigynal teeth. 


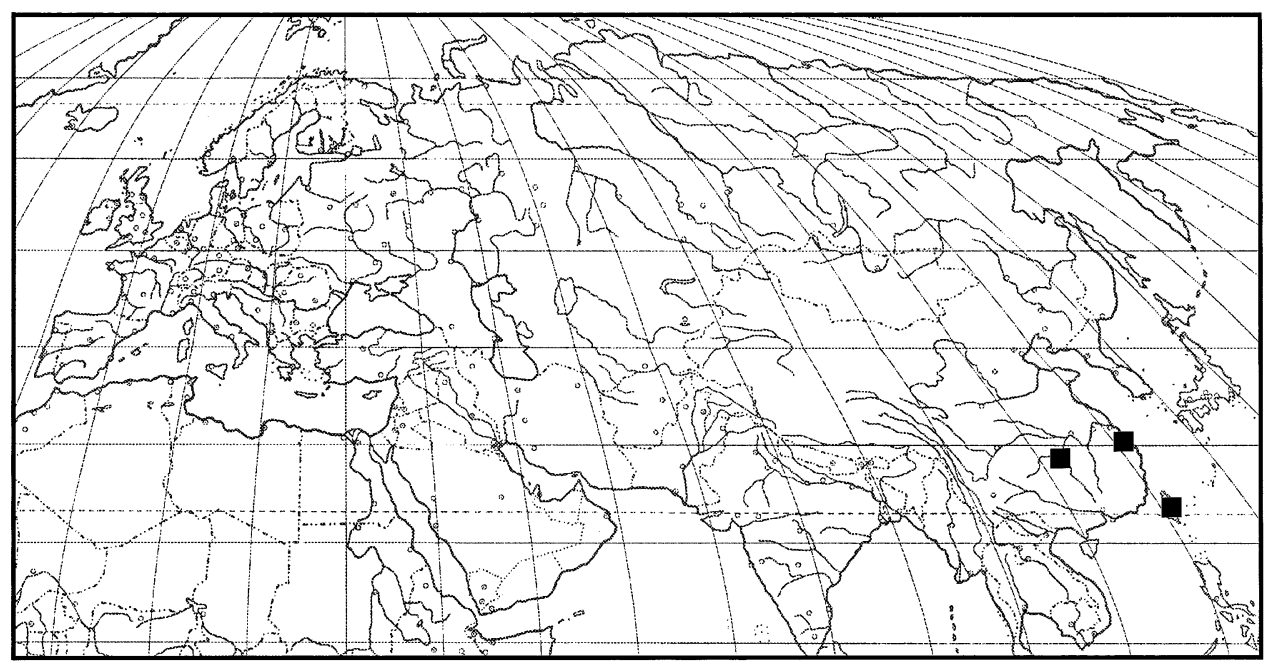

Map 11. Records of Leptocoelotes.

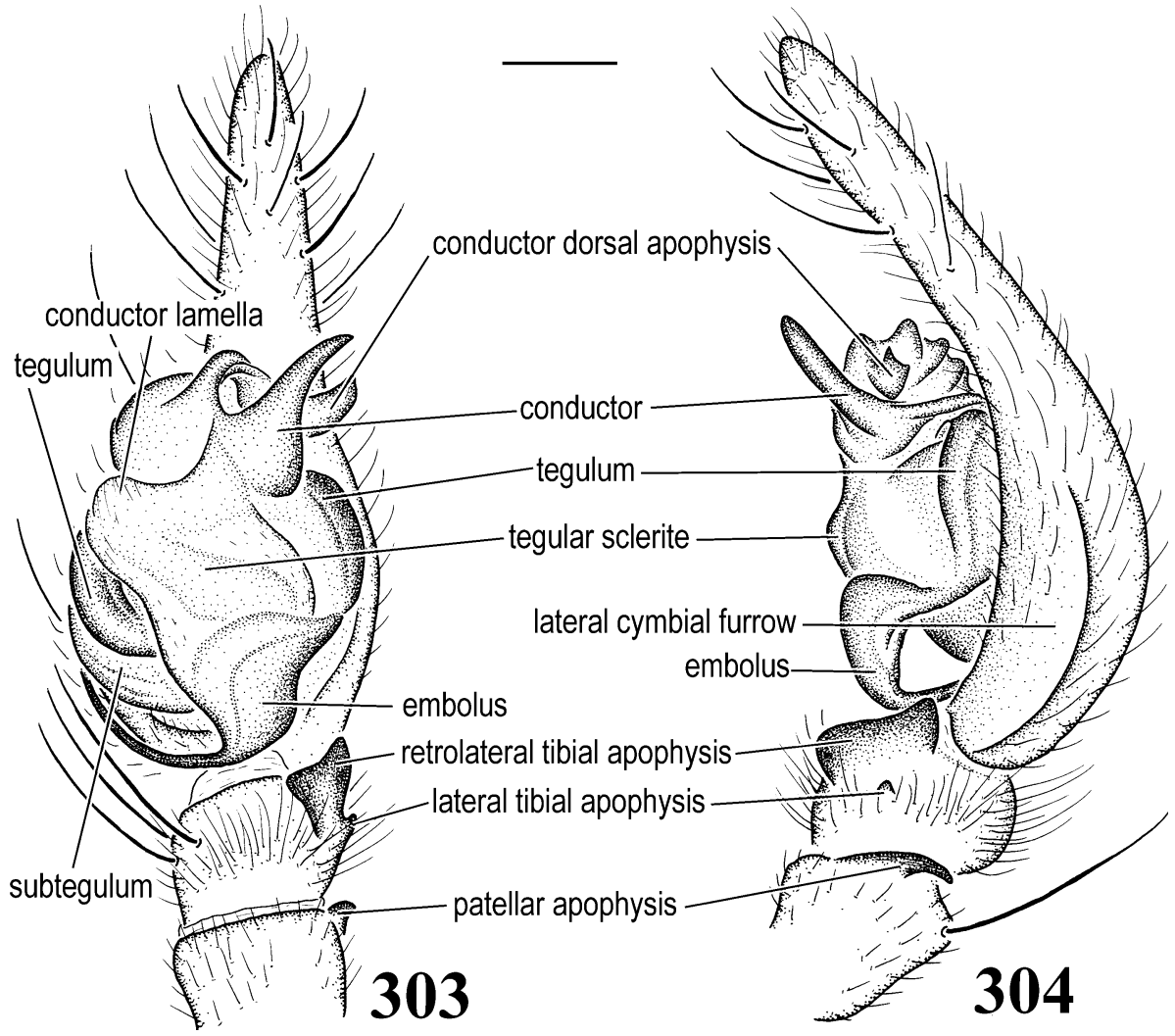

Figs. 303, 304. Leptocoelotes pseudoluniformis (Zhang, Peng and Kim), male palp. 303. Ventral view. 304. Retrolateral view. 

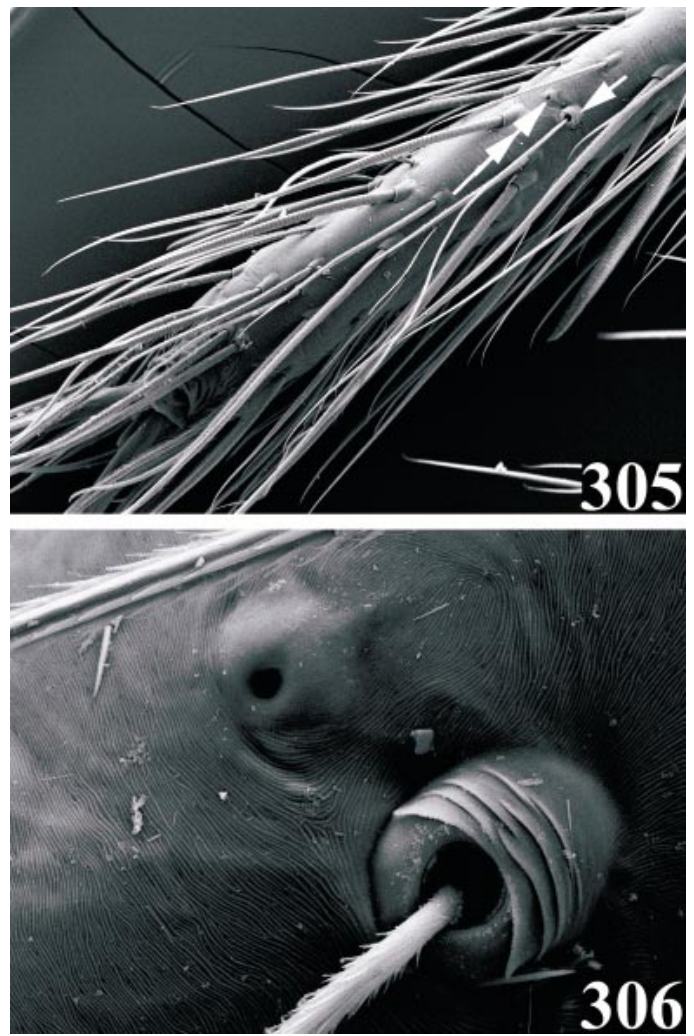

Figs. 305, 306. Leptocoelotes pseudoluniformis (Zhang, Peng and Kim), female. 305. Tarsus, showing position of trichobothria (single arrow) and tarsal organ (double arrows). 306. Trichobothrium and tarsal organ.

DiagnOSIS: Females of this species can be easily recognized by the relatively short, broad epigynal teeth, the shape of the epigynum, and the medially situated copulatory ducts (figs. 294, 295).

DESCRIPTION: Female (paratype): Total length 6.30. Carapace 3.10 long, 1.80 wide. Abdomen dried, 3.20 long, 1.80 wide. Eye sizes and interdistances: AME 0.09, ALE 0.18, PME 0.17, PLE 0.17; AME-AME 0.08, AME-ALE 0.09, PME-PME 0.09, PME-PLE 0.11, AME-PME 0.12. Clypeus height 0.15. Leg measurements: I, 7.37 (2.10, 2.49, 1.70, $1.08)$; II, 6.48 (1.92, 2.10, 1.51, 0.95); III, $6.04(1.70,1.94,1.60,0.80)$; IV, 8.37 (2.28, $2.70,2.30,1.09)$. Epigynal teeth relatively short, broad, anteriorly situated; atria more or less separated by median septum; copulatory
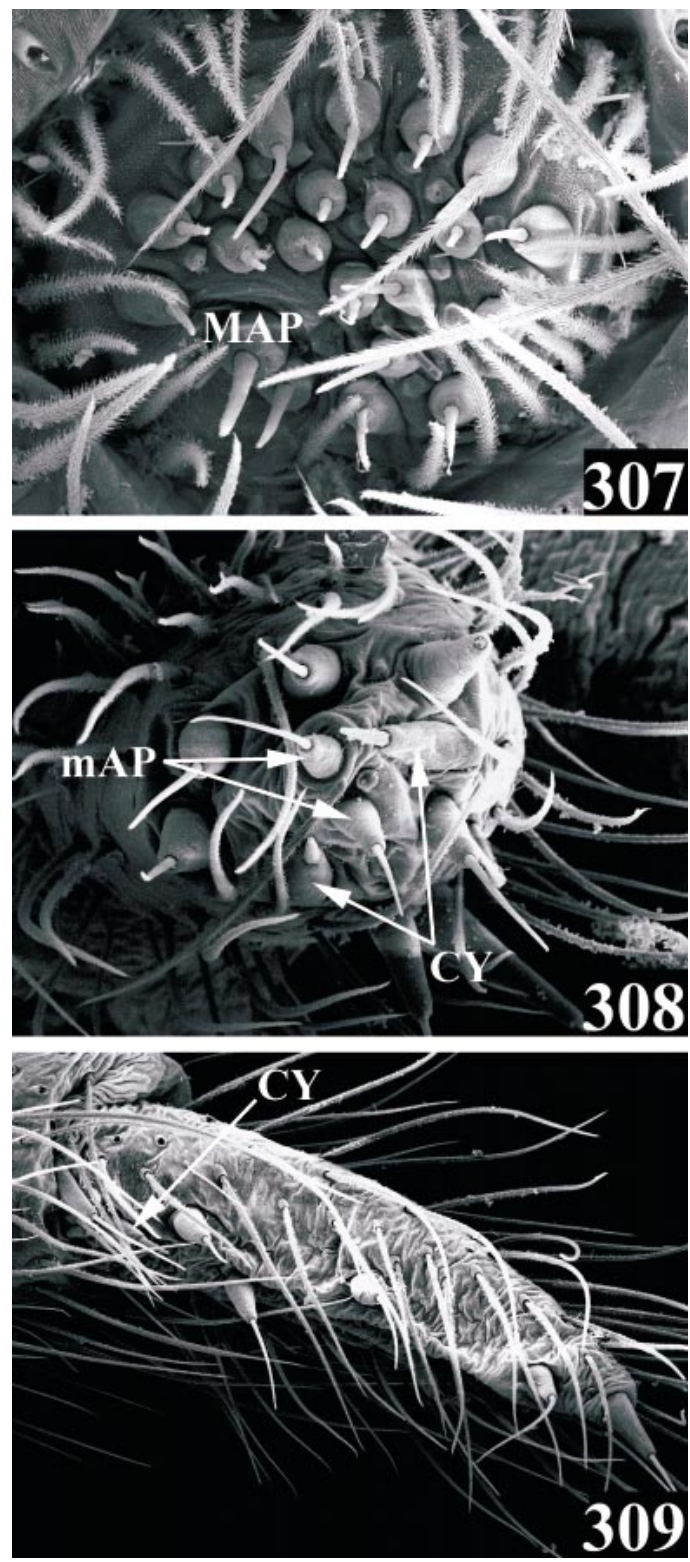

Figs. 307-309. Leptocoelotes pseudoluniformis (Zhang, Peng and Kim), female spinnerets, ventral view, left. 307. ALS. 308. PMS. 309. PLS. Abbreviations are spelled out on p. 6.

ducts short, situated mesally, anteriorly extended; spermathecal heads short, situated mesally; spermathecae broad, convoluted, wide apart posteriorly and anteriorly adjacent (figs. 294, 295).

Male: Unknown. 


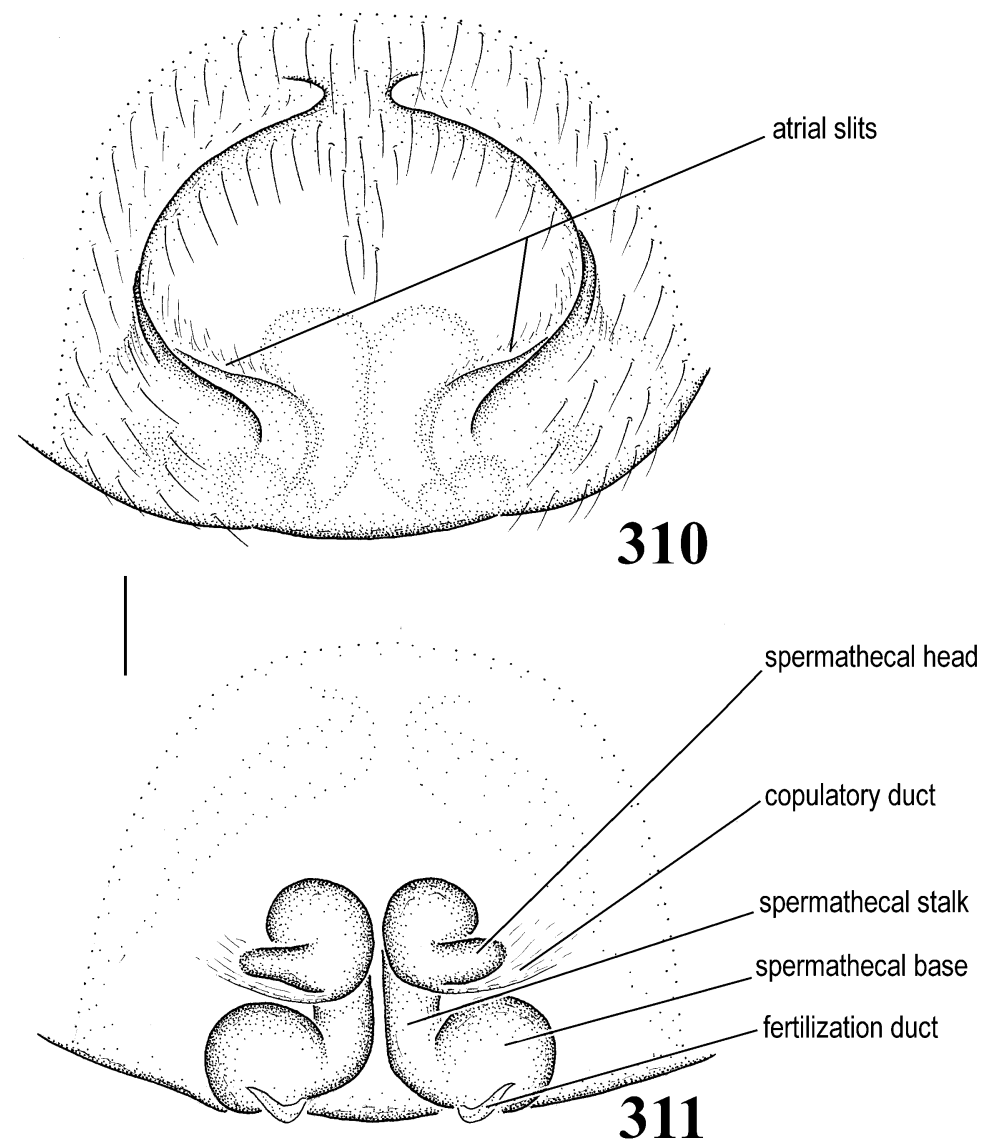

Figs. 310, 311. Longicoelotes karschi, new species, female. 310. Epigynum. 311. Vulva.

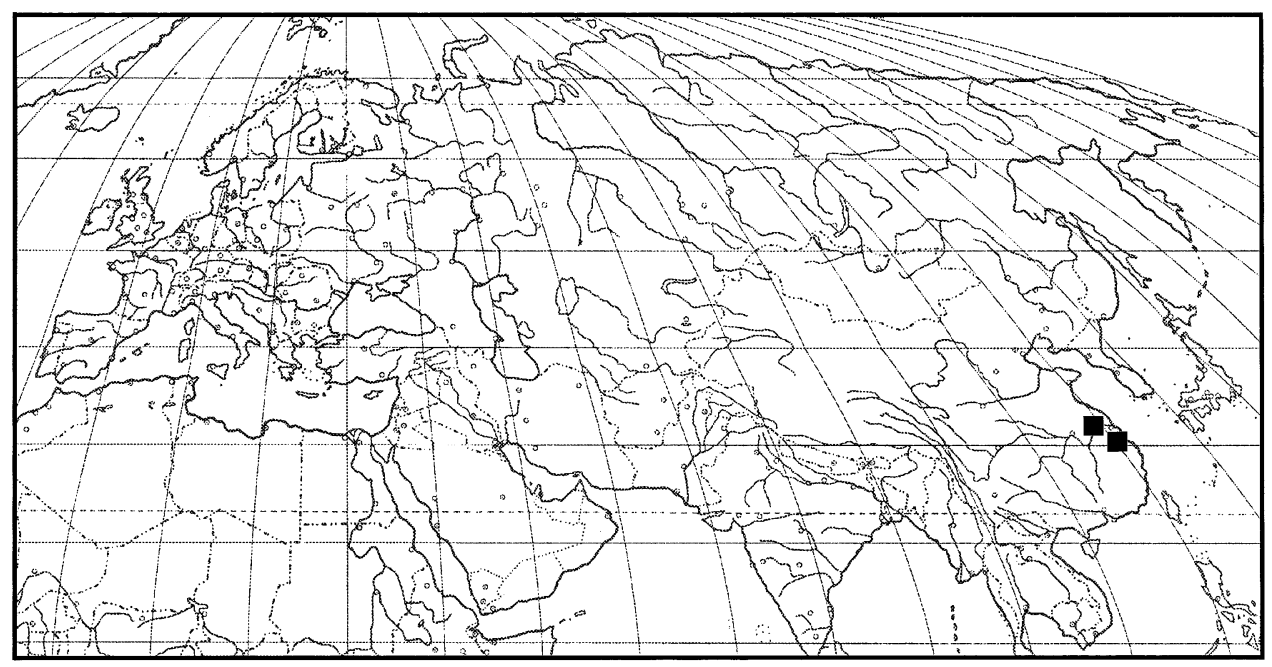

Map 12. Records of Longicoelotes. 


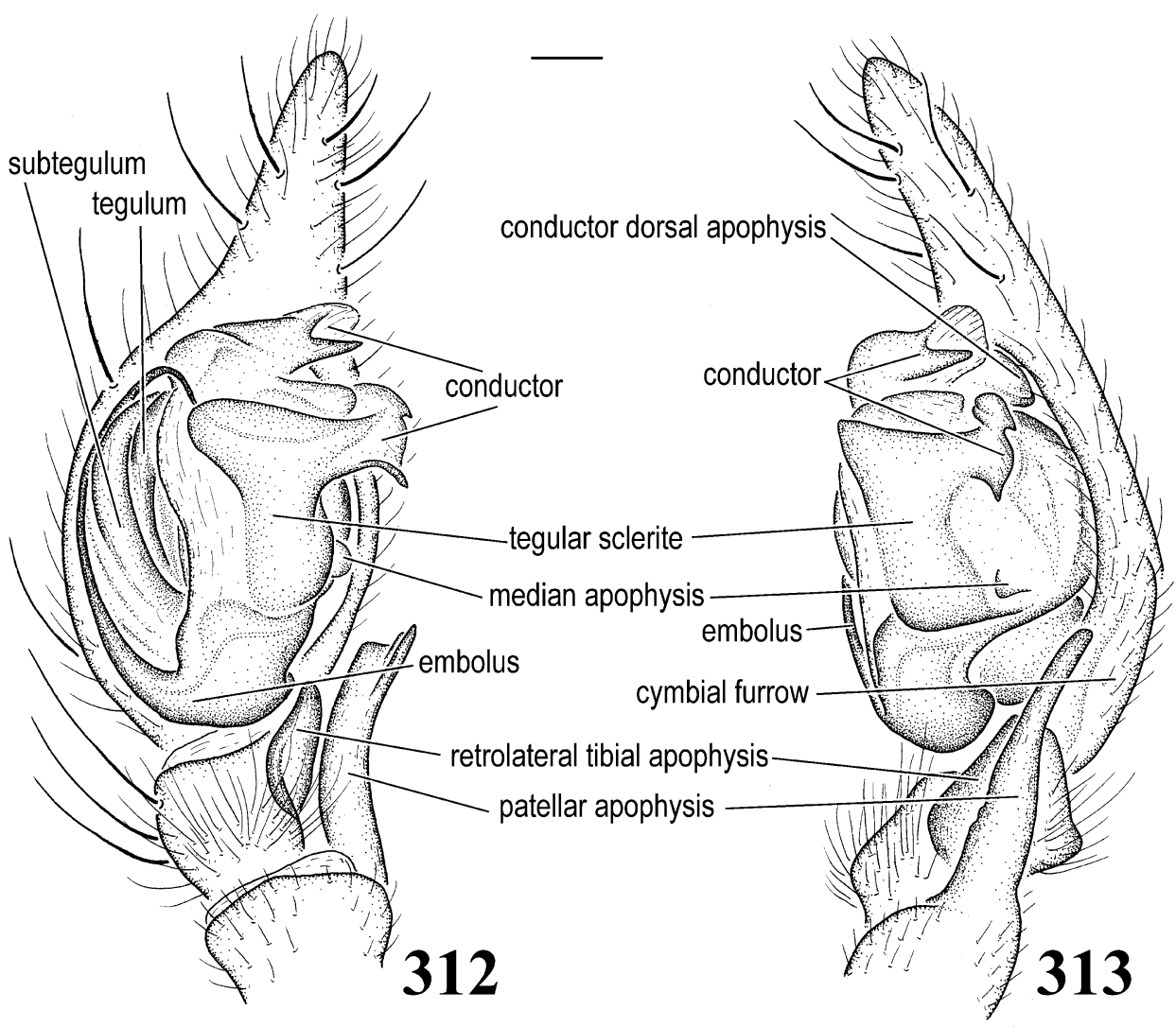

Figs. 312, 313. Longicoelotes karschi, new species, male palp. 312. Ventral view. 313. Retrolateral view, showing conductor dorsal apophysis.

Other Material Examined: None. DisTRIBUTION: Nepal.

\section{LEPTOCOELOTES, NEW GENUS}

TyPE SPECIES: Coelotes pseudoluniformis Zhang, Peng and Kim, 1998.

ETYMOLOGY: The generic name is derived from its similarity to Coelotes and the weak epigynal teeth, and is masculine in gender.

DiAGNOSIS: Females can be easily recognized by the broad but weakly sclerotized epigynal teeth, a shallow atrium, and short copulatory ducts (figs. 296-299), and males by the complex conductor, the absence of a conductor dorsal apophysis, and the absence of median apophysis (figs. 302-304). Both sexes have four to five promarginal and retromarginal teeth (fig. 301).

DESCRIPTION: See description of type species (below).
DisTRIBUTION: China (map 11).

Composition: Two species are included.

1. Leptocoelotes edentulus (Wang and Ono, 1998): female holotype from Ilan, Taiwan, in NSMT, examined. NEW COMBINATION (transferred here from Coelotes).

2. Leptocoelotes pseudoluniformis (Zhang, Peng and Kim, 1998): female holotype from Tiantong, Zhejiang, China, in HBI, examined. NEW COMBINATION (transferred here from Coelotes).

Leptocoelotes pseudoluniformis (Zhang, Peng, and Kim)

Figures 296-309

Coelotes pseudoluniformis Zhang, Peng and Kim, 1998: 293, figs. 6, 7 (female holotype from Tiantong, Zhejiang, China, in HBI, examined).

DiAGNOSIS: Female of this species can be recognized from $L$. edentus by the laterally 


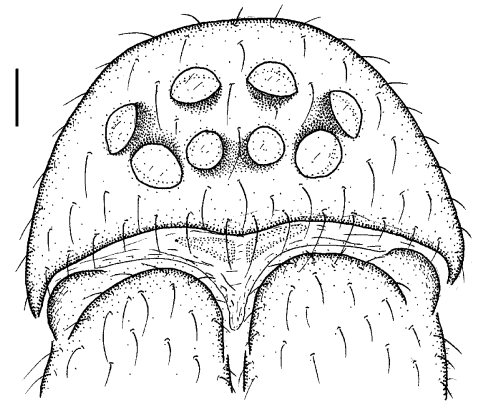

315

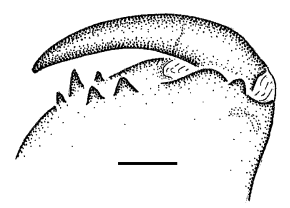

316

lateral tibial apophysis

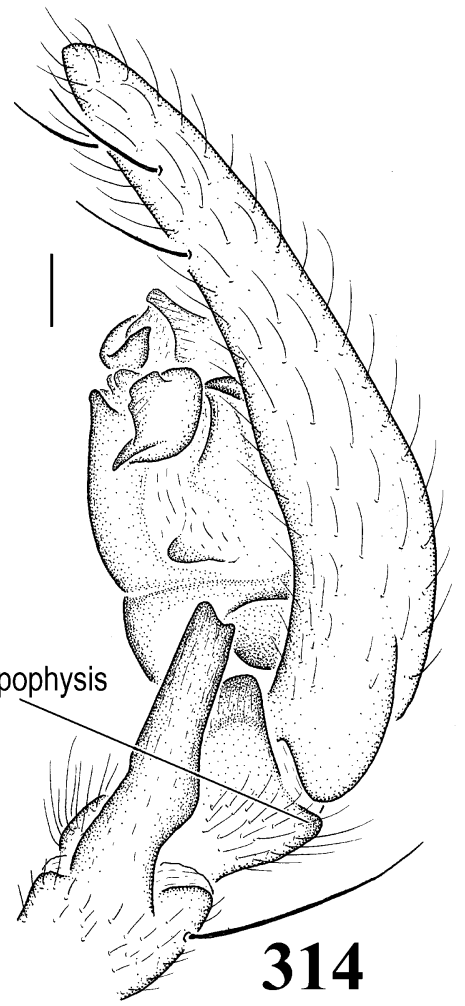

Figs. 314-316. Longicoelotes karschi, new species. 314. Male palp, retrolateral view. 315. Carapace, front view. 316. Chelicera, ventral view.

situated hoods, the anterior origin of the copulatory ducts, and the relatively elongated spermathecae (figs. 296-299), and males by the tiny lateral tibial apophysis, the strongly bifid conductor, and the absence of both a conductor dorsal apophysis and a median apophysis (figs. 302-304).

DESCRIPTION: Total length about 5.008.50. From front, anterior eye row slightly procurved, posterior row procurved; eye sizes and arrangements: AME smallest and slightly smaller than ALE, ALE subequal to PLE and PME; AME-AME slightly shorter than AME diameter, ALE-AME close together, subequal to ALE-PLE, PME-PLE subequal to PME-PME, about PME radius, AME-PME about PME radius. Clypeal height about 1.5-2 times AME diameter; chilum divided, elongated (fig. 300). Chelicerae with five promarginal and four to five retromarginal teeth (fig. 301). Labium with width slightly wider than length. Length of 1st leg patella + tibia longer than carapace length.

Tarsal organ situated away from distal end of tarsus, about same position as most distal trichobothrium (figs. 305, 306). Female spinnerets with apex of ALS with 2 major ampullate gland spigots (MAP), 19 piriform gland spigots; PMS with 1 or 2 minor ampullate gland spigots (mAP), 7 aciniform gland spigots, 2 cylindrical gland spigots (CY); PLS with about 12 aciniform gland spigots and 2 cylindrical spigots (figs. 307309).

Epigynum with epigynal teeth broad but weakly sclerotized; atrium broad but shallow, not apparent; copulatory ducts short, situated anterolaterad of spermathecae; spermathecal heads situated anterad of spermathecae; spermathecae slightly elongated, convoluted, widely separated, with indistinct bases and stalks (figs. 296-299). Male palpal patellar apophysis small, strongly curved dorsally; 



Figs. 317-319. Longicoelotes karschi, new species, female. 317. Tarsus, showing position of tarsal organ (double arrows) and distalmost trichobothrium (single arrow). 318. Trichobothrium. 319. Tarsal organ.

RTA small, with distal end extended beyond tibia; lateral tibial apophysis tiny; cymbial furrow moderately long; conductor complex; conductor dorsal apophysis absent; conductor lamella short; embolus basal in origin,
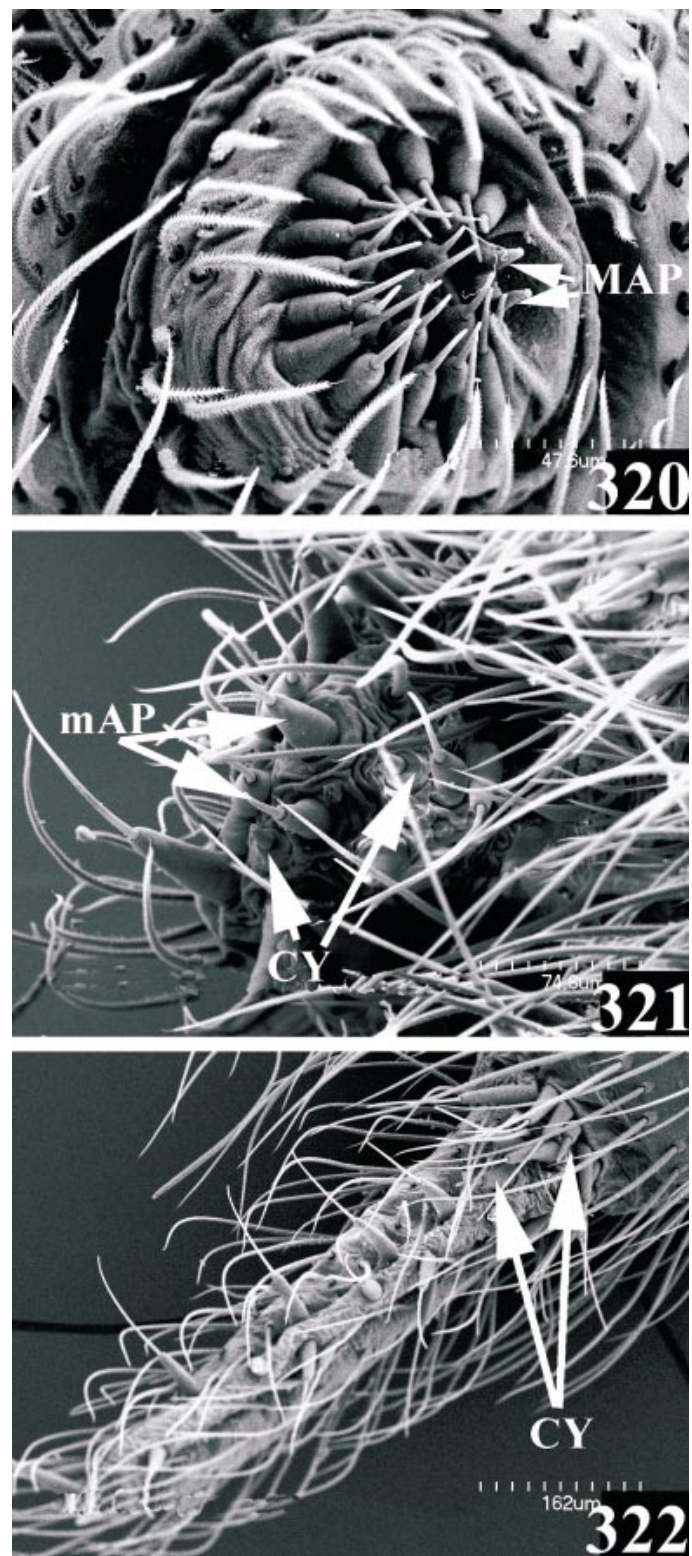

Figs. 320-322. Longicoelotes karschi, new species, female spinnerets, ventral view, left. 320. ALS. 321. PMS. 322. PLS. Abbreviations are spelled out on p. 6 .

moderately long, linear; median apophysis absent (figs. 302-304).

MAterial ExAmined: CHINA: Zhejiang: Tiantong, January 20, 1988, female holotype (Y. J. Zhang, HBI). Hunan: Changsha, Yuelushan, December 22, 1982, 2 males and 4 females (J. F. Wang, HBI). 


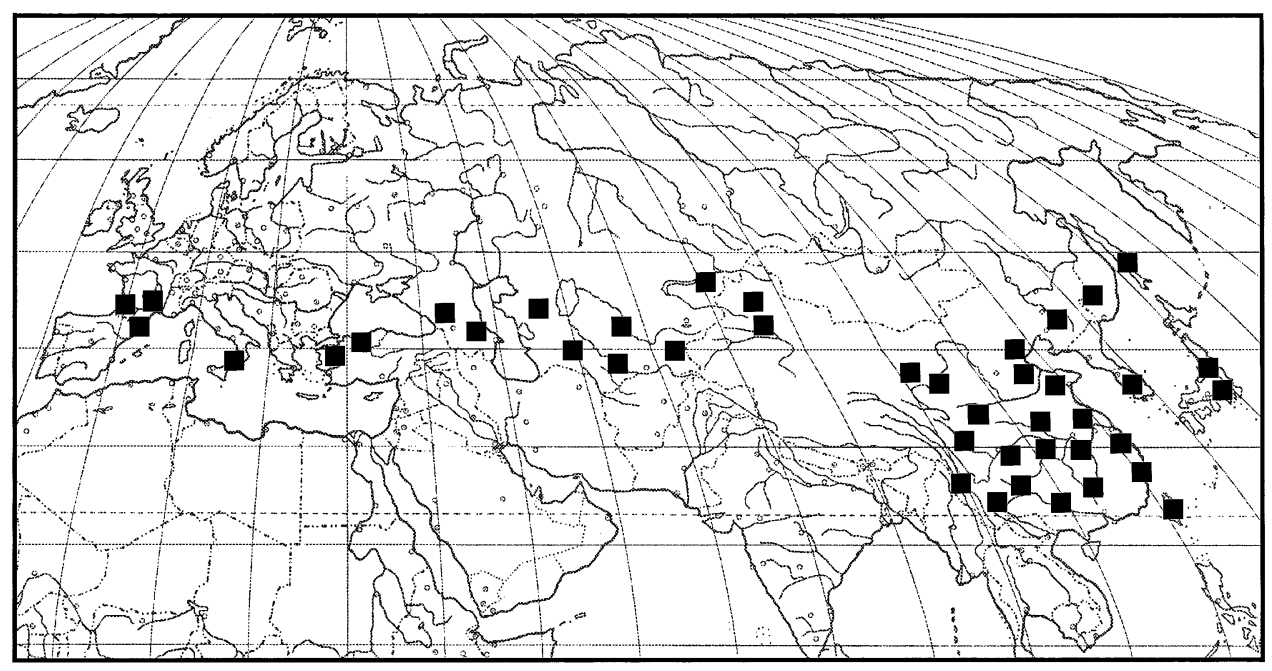

Map 13. Records of Paracoelotes.

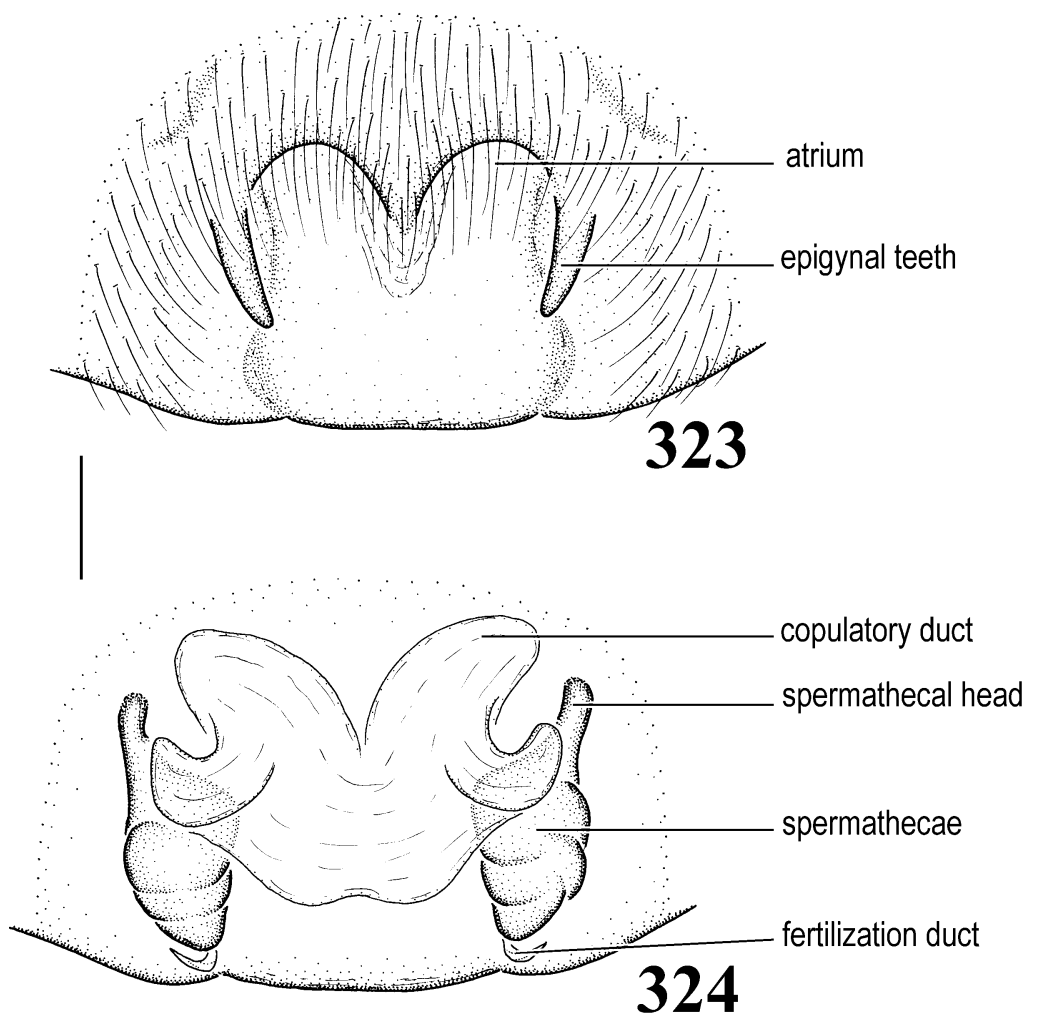

Figs. 323, 324. Paracoelotes armeniacus (Brignoli), female. 323. Epigynum. 324. Vulva. 


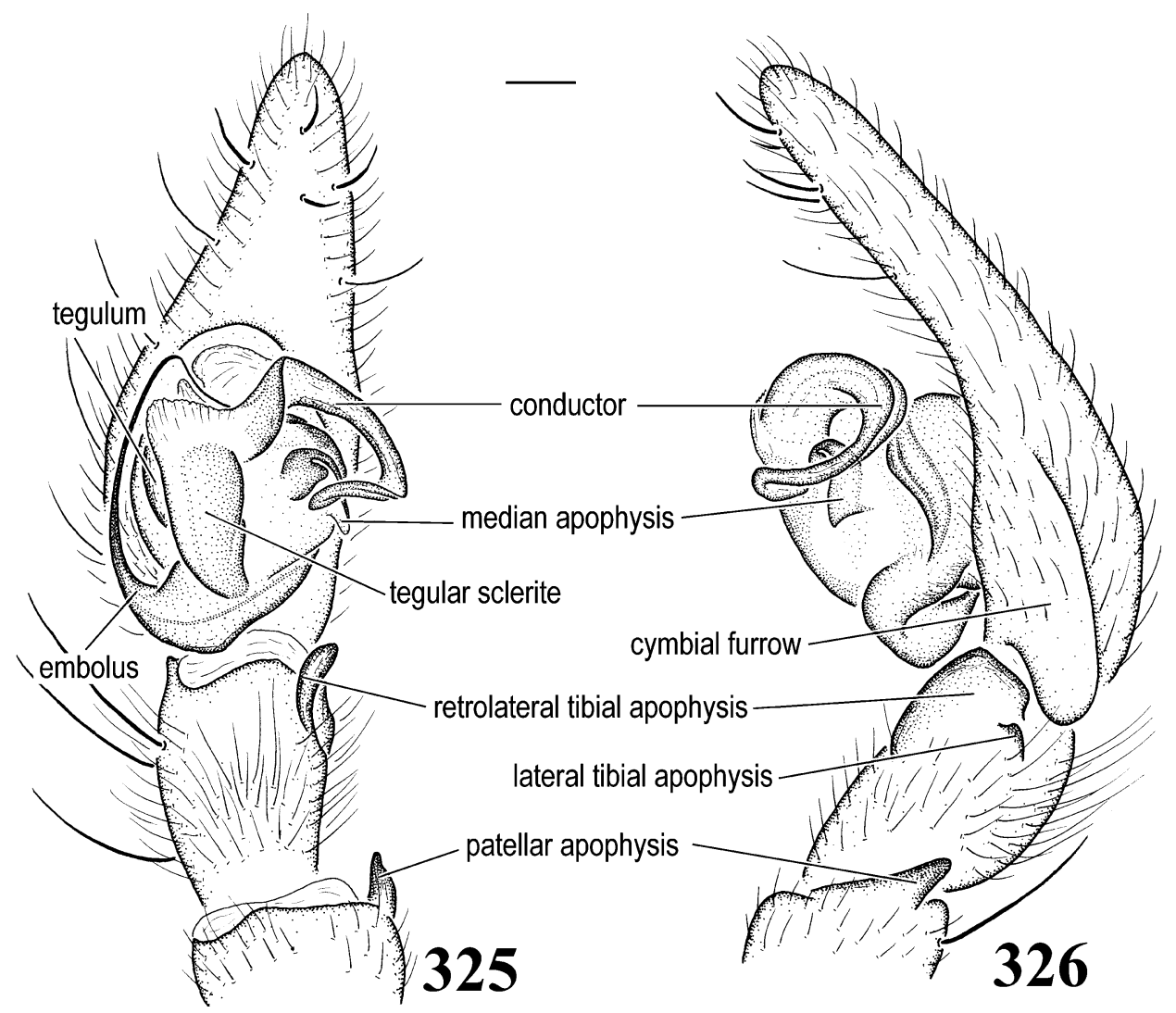

Figs. 325, 326. Paracoelotes armeniacus (Brignoli), male palp. 325. Ventral view. 326. Retrolateral view.

Distribution: China (Zhejiang, Hunan).

\section{LONGICOELOTES, NEW GENUS}

TyPE SPeCIEs: Longicoelotes karschi Wang, new species.

ETYMOLOGY: The generic name is derived from its similarity to Coelotes and to the strongly elongated patellar apophysis, and is masculine in gender.

DiAGNOSIS: Females can be easily recognized by the absence of epigynal teeth and the distinct shape of the epigynum (figs. 310, 311 ), and males by the strongly elongated patellar apophysis, the complex conductor, and the absence of median apophysis (figs. 312-314).

DESCRIPTION: See description of type species (below).

Distribution: China (map 12).
Composition: Only the type species.

Longicoelotes karschi, new species Figures 310-322

Coelotes mollendorffi: Schenkel, 1963: 280, fig. 158. - Chen and Zhang, 1991: 187, fig. 185 (misidentification).

Coelotes moellendorffi: Song et al., 1999: 376, figs. 221E, 221F, 222M, 224A (misidentification).

TYPE: Male holotype from Zijin (Purple) Mt., 350-450 m, Nanjin, China (September 13, 1997; X. P. Wang), and female paratype from same locality (October 9, 1988; P. Beron), deposited in AMNH.

DiAgNosis: This species can be easily identified by the absence of epigynal teeth, the distinct shape of the epigynum, the short copulatory ducts, and the distinct spermathe- 


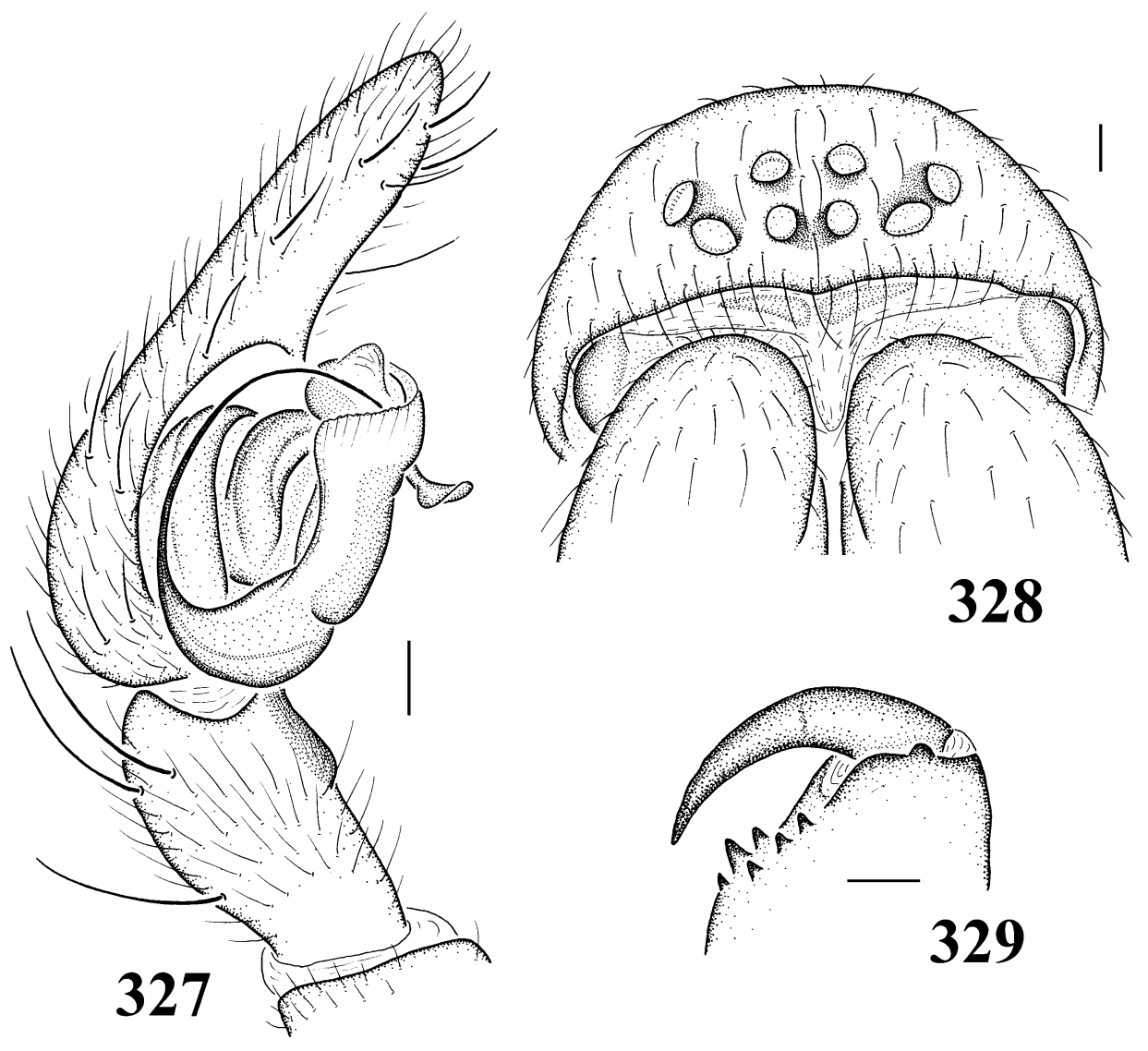

Figs. 327-329. Paracoelotes armeniacus (Brignoli). 327. Male palp, prolateral view. 328. Eyes, dorsal view. 329. Chelicera, ventral view.

cal stalks and bases of females (figs. 310, 311 ), and by the strongly elongated patellar apophysis, complex conductor, and the small median apophysis of males (figs. 312-314).

DESCRIPTION: Male (holotype): Total length 7.70. Eye sizes and interdistances: AME 0.15, ALE 0.18, PME 0.18, PLE 0.17, AME-AME 0.08, AME-ALE 0.03, ALEPLE 0.03, PME-PME 0.10, PME-PLE 0.12, AME-PME 0.15. Leg measurements: I, 13.5 (3.42, 4.46, 3.40, 2.18); II, 12.1 (3.20, 3.84, $3.10,1.92)$; III, 11.3 (3.00, 3.40, 3.26, 1.62); IV, 15.6 (4.00, 4.80, 4.60, 2.20). Male palp with patellar apophysis strongly elongated; RTA long, with distal end slightly extended beyond tibia; lateral tibial apophysis situated relatively dorsally; cymbial furrow short; conductor short, broad, bifurcated, with dor- sal one broad and ventral one slender; conductor lamella less developed; conductor dorsal apophysis broad; median apophysis small, not spoonlike; embolus basal in origin, moderately long, linear (figs. 312-314).

Female (paratype): Total length 7.80. Eye sizes and interdistances: AME 0.15, ALE 0.20, PME 0.19, PLE 0.19, AME-AME 0.10, AMEALE 0.04, ALE-PLE 0.05, PME-PME 0.11, PME-PLE 0.17, AME-PME 0.16. Leg measurements: I, 12.1 (3.24, 4.20, 2.86, 1.82); II, $10.8(3.00,3.60,2.60,1.58)$; III, 10.1 (2.80, $3.10,2.72,1.44)$; IV, 13.9 (3.60, 4.46, 4.00, 1.80). Clypeal height roughly twice AME diameter or slightly shorter; chilum divided, elongated (fig. 315). Chelicerae with three promarginal, two retromarginal teeth (fig. 316). Labium slightly wider than long. Length of fe- 

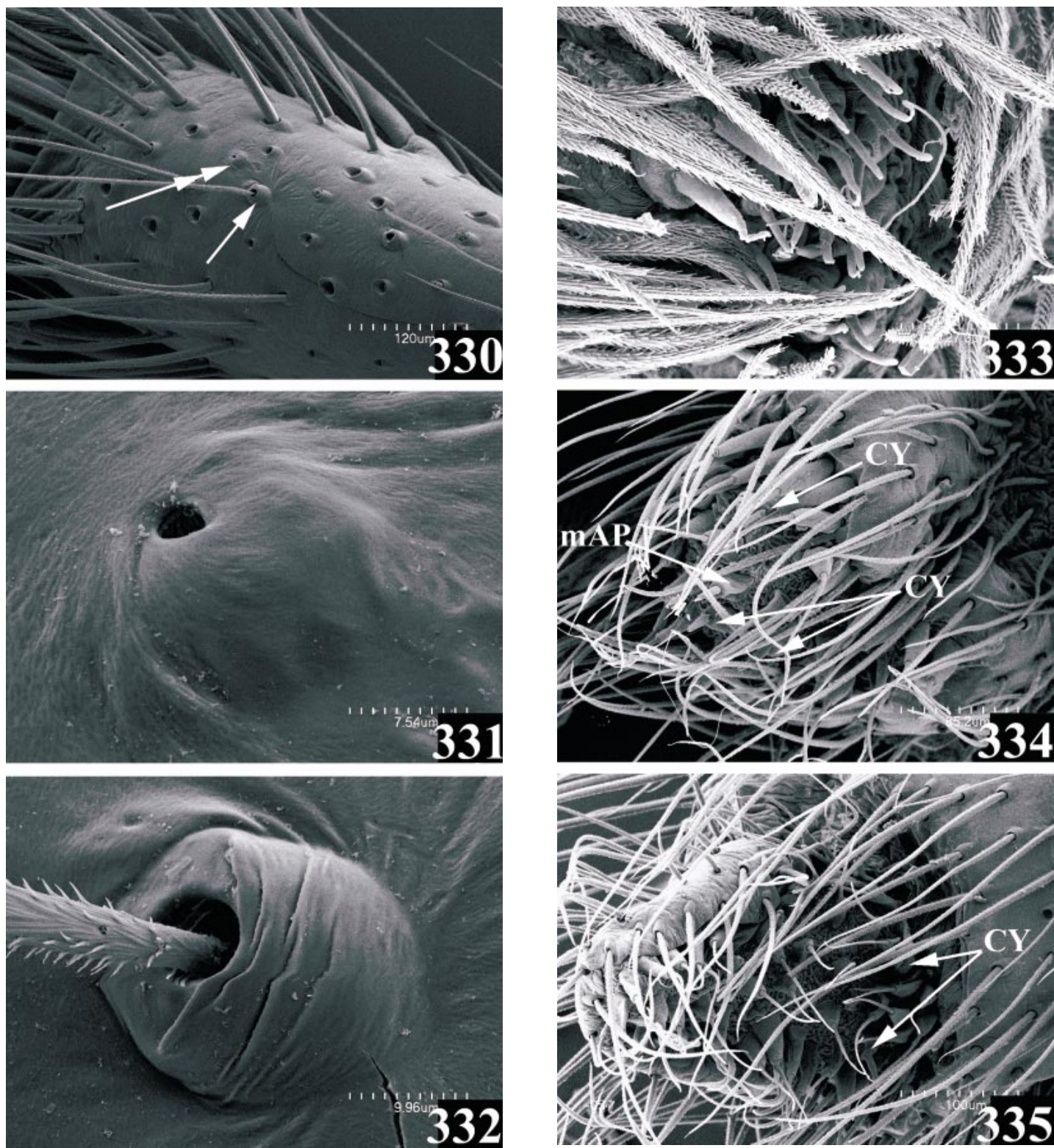

Figs. 330-332. Paracoelotes armeniacus (Brignoli), female. 330. Tarsus, showing position of tarsal organ (double arrows) and the distalmost trichobothrium (single arrow). 331. Tarsal organ. 332. Trichobothrium.

male 1st leg patella + tibia longer than carapace length. Epigynum with epigynal teeth absent; middle part of epigynum relatively elevated with clear lateral edges; atrium reduced, not apparent; copulatory ducts short; sperma-

Figs. 333-335. Paracoelotes armeniacus (Brignoli), female spinnerets, ventral view. 333. ALS, right. 334. PMS, left. 335. PLS, right. Abbreviations are spelled out on p. 6 .

thecal heads pointed laterally; spermathecae with distinct stalks and bases; spermathecal stalks situated medially, close to each other; spermathecal bases large, widely separated (figs. 310, 311).

Tarsal organ situated close to distal end of 


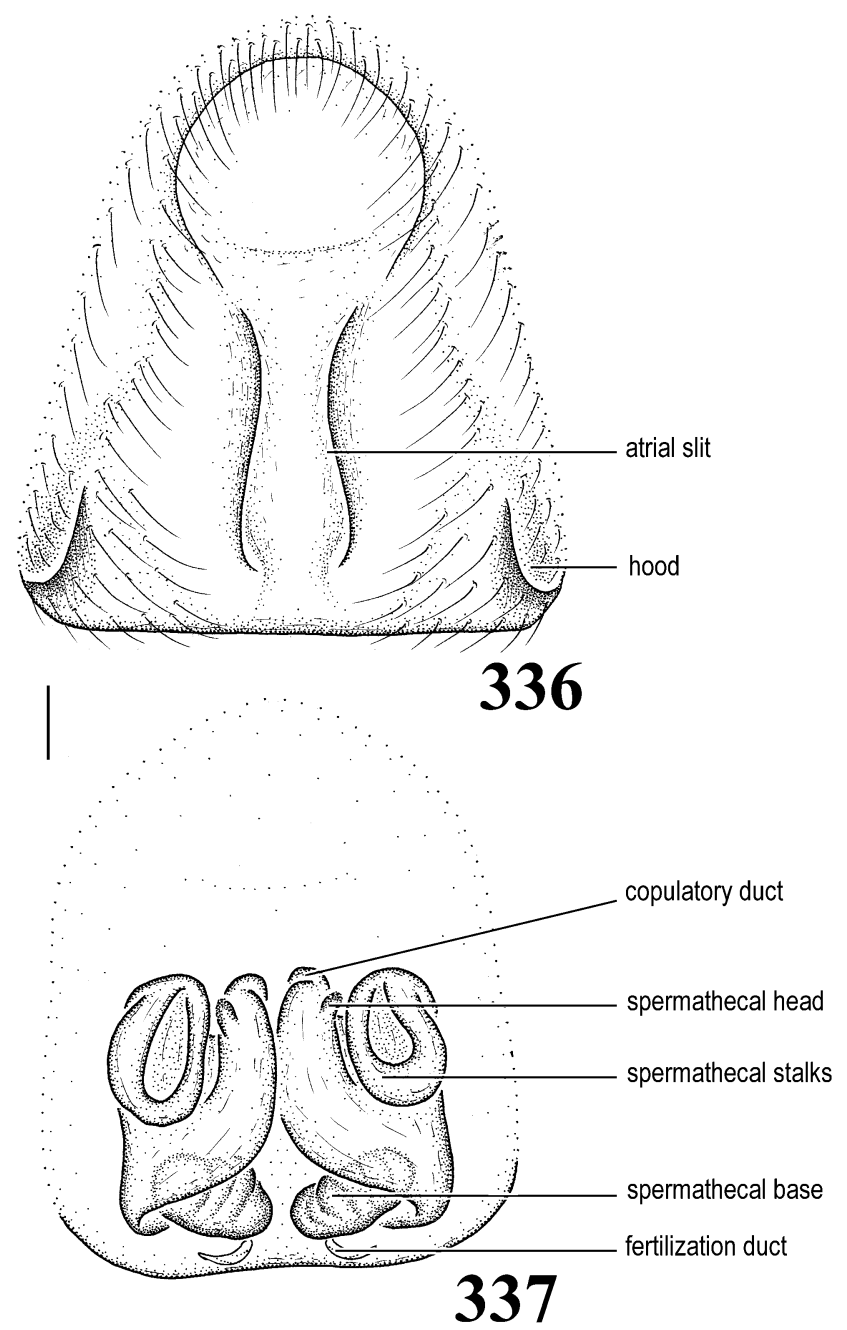

Figs. 336, 337. Platocoelotes impletus (Peng and Wang), female. 336. Epigynum. 337. Vulva.

tarsus, slightly anterior of most distal trichobothrium (figs. 317-319). Only female spinnerets examined: apex of ALS with 2 major ampullate gland spigots (MAP), about 25-31 piriform gland spigots; PMS with 1 or 2 minor ampullate gland spigots (mAP) and about 16-20 aciniform gland spigots, 2 cylindrical gland spigots (CY); PLE with about 22 aciniform gland spigots and 2 cylindrical gland spigots (figs. 320-322).

NotEs: Examination of the types of $C$. mollendorffi (3 female syntypes from Peking, China, in ZMB, examined) shows that $C$. mollendorffi is in fact a junior synonym of Paracoelotes spinivulvus (Simon, 1880).
Other Material Examined: CHINA: Zhejiang: West Tschenkiang, April 1872, 1 female (A. David, MNHN, B2011 bis); Hangtscheou, 1925, 3 females (MNHN, B2011 bis); Lin-An, October 19, 1974, 3 males and 3 females (C. D. Zhu, NBUMS).

Distribution: China (Jiangsu, Zhejiang).

\section{PARACOELOTES BRIGNOLI}

Paracoelotes Brignoli, 1982: 348. - Platnick, 1989: 425; - Platnick, 1993: 577; - Platnick, 1997: 669.

Type Species: Coelotes armeniacus Brignoli, 1978 , by original designation. 


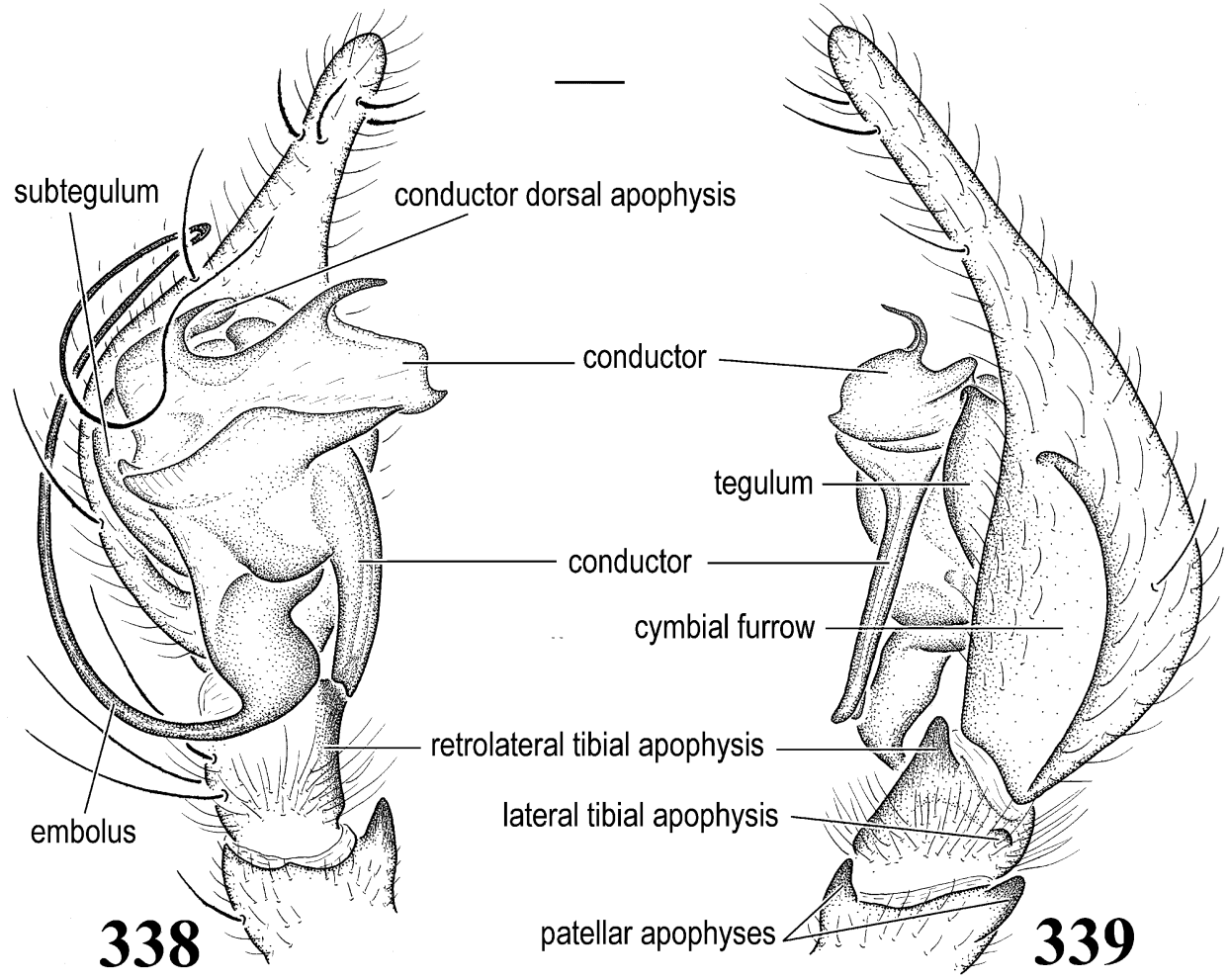

Figs. 338, 339. Platocoelotes impletus (Peng and Wang), male palp. 338. Ventral view. 339. Retrolateral view.

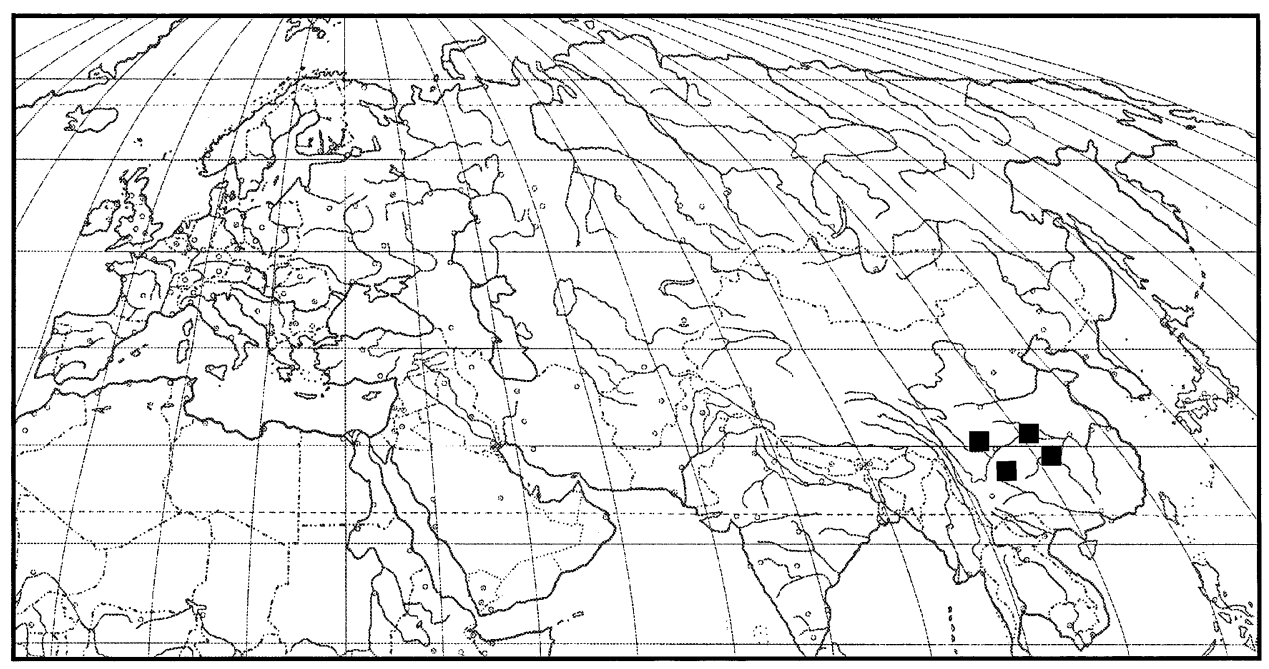

Map 14. Records of Platocoelotes. 

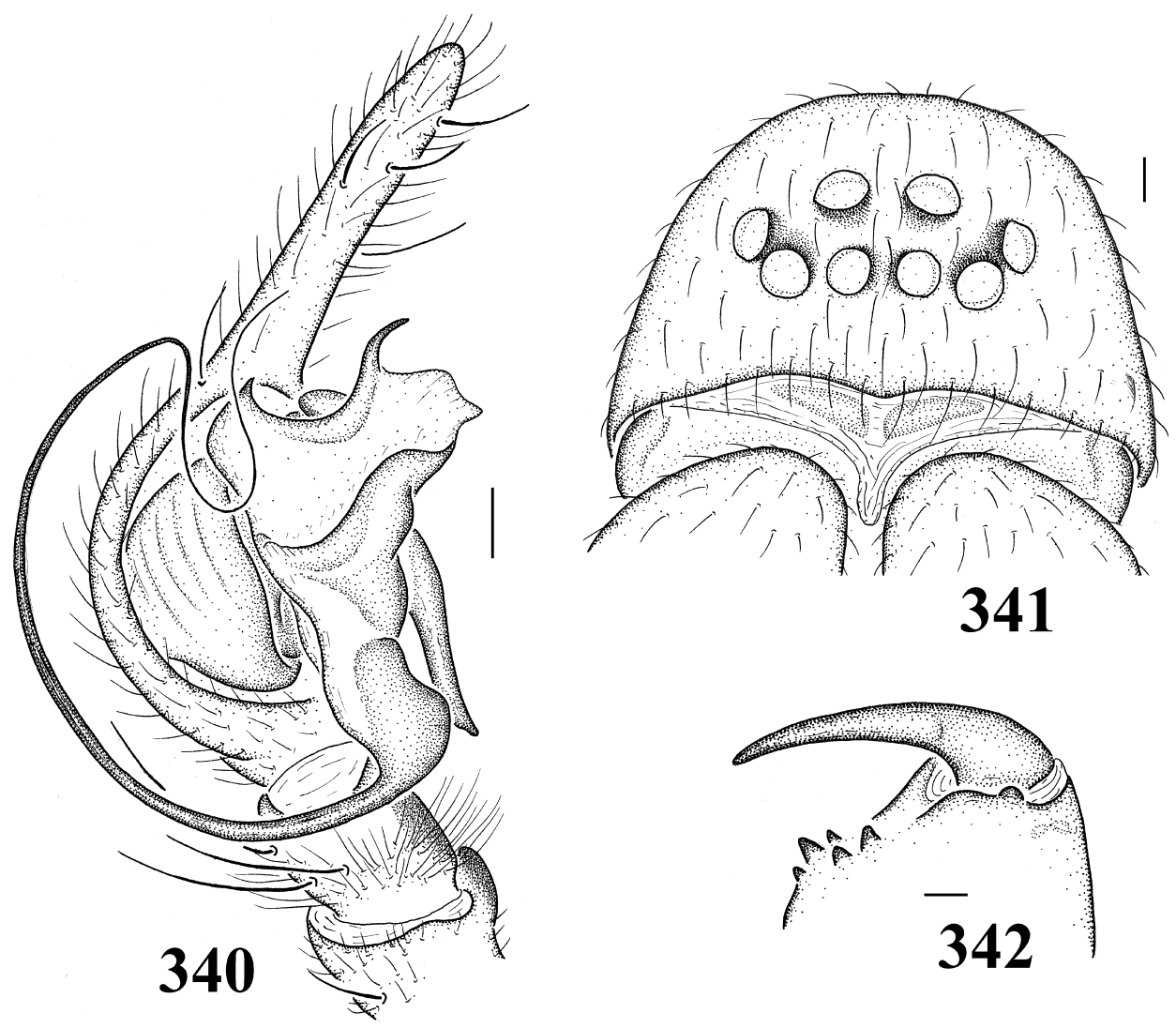

341

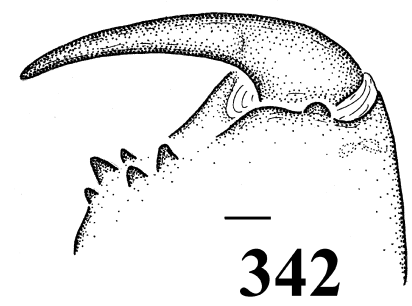

Figs. 340-342. Platocoelotes impletus (Peng and Wang), male palp. 340. Prolateral view. 341. Carapace, front view. 342. Chelicera, ventral view.

DiAgNOSIS: Females can be recognized by the large epigynal atrium, the long, laterally situated epigynal teeth, and the broad copulatory ducts (figs. 323, 324), and males by the long, looped conductor and the absence of a conductor dorsal apophysis (figs. 325-327).

DESCRIPTION: See description of type species (below).

Distribution: Europe and Asia (map 13).

Composition: The genus Paracoelotes comprises 16 species, including 5 new combinations.

1. Paracoelotes armeniacus (Brignoli, 1978): male holotype, male and female paratypes from Turkey, in MCV and MNHG, examined.

2. Paracoelotes bidens (Caporiacco, 1935): type depository unknown.

3. Paracoelotes cottarellii (Brignoli, 1978): female holotype and paratypes from Sinop, Turkey, in MHNG and MCV, exam- ined. NEW COMBINATION (transferred here from Coelotes).

4. Paracoelotes fedotovi (Charitonov, 1946): female holotype from Ishkent, Uzbekistan, depository unknown, not examined. Females from Uzbekistan, in PSU and AMNH, examined.

5. Paracoelotes garibaldii (Kritscher, 1969): female holotype from Cippo Garibaldi, Aspromonte, Italy, in MCV, examined.

6. Paracoelotes involutus (Wang et al., 1990): male and female paratypes from Xishan, Kuming, Yunnan, China, in HBI, examined. NEW COMBINATION (transferred here from Coelotes).

7. Paracoelotes luctuosus (L. Koch, 1878): type depository unknown. Males and females from East Asia, in AMNH, HBI, IZB, CAS, SMF, and ZMB, examined.

8. Paracoelotes luniformis (Zhu and Wang, 1994): female holotype, male and fe 

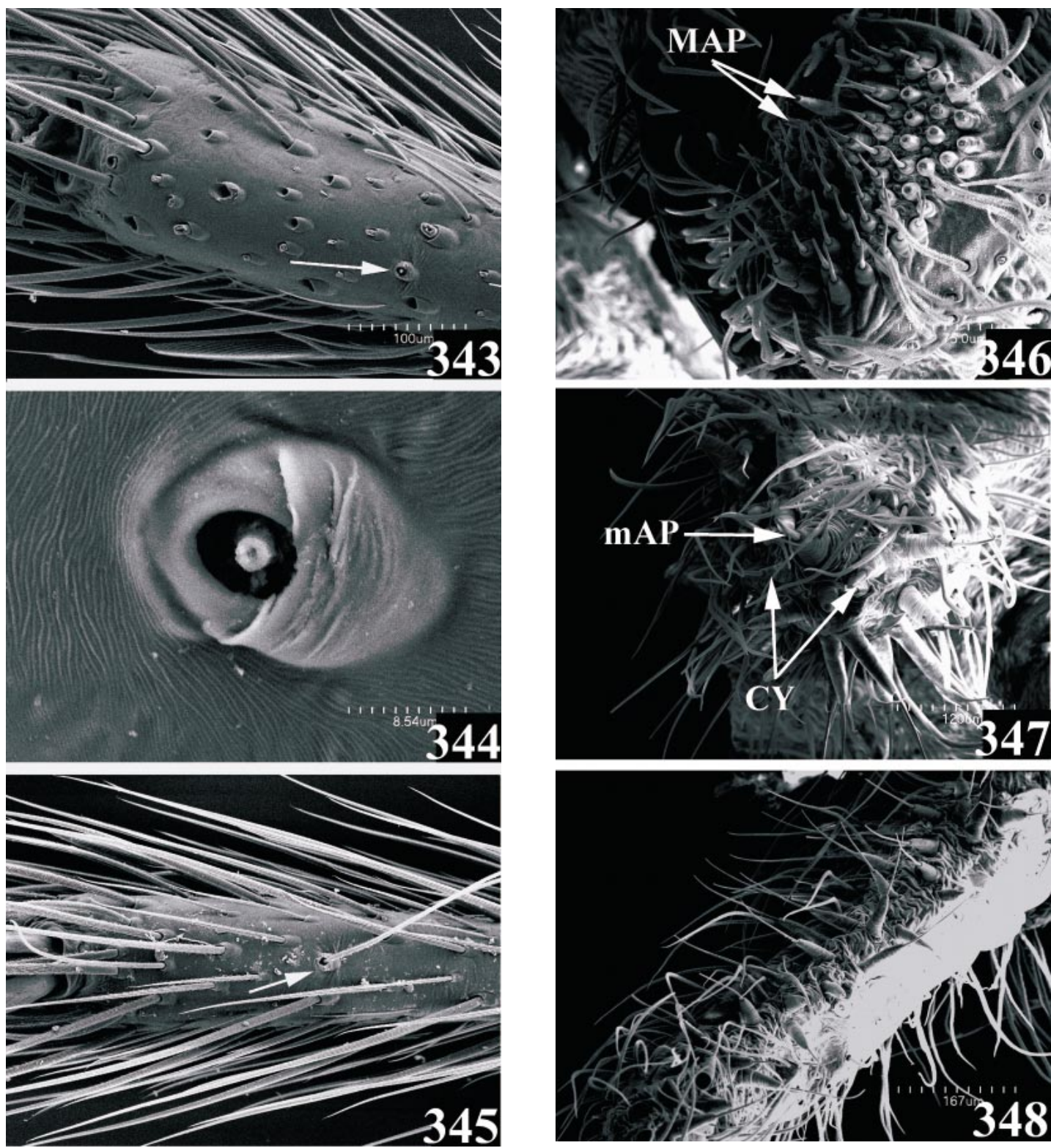

Figs. 343-345. Platocoelotes impletus (Peng and Wang). 343. Tarsus, with tarsal organ not visible. 344. Trichobothrium. 345. Tarsus (another specimen), with tarsal organ not visible.

male paratypes from Xiaguan, Yunnan, China, in NBUMS, not examined. NEW COMBINATION (transferred here from Coelotes). Males and females from Yunnan, China, in CAS and HBI, examined.

9. Paracoelotes major (Kroneberg, 1875): type depository unknown. Female specimens

Figs. 346-348. Platocoelotes impletus (Peng and Wang), female spinnerets, ventral view, right. 346. ALS. 347. PMS. 348. PLS. Abbreviations are spelled out on p. 6.

from Middle Asia, in AMNH and PSU, examined.

10. Paracoelotes pyrenaeus (Simon, 1870): male lectotype and female paralectotype from Montlouis, France, in MNHN, examined.

11. Paracoelotes segestriformis (Defour, 1820): male neotype and female paraneotype 

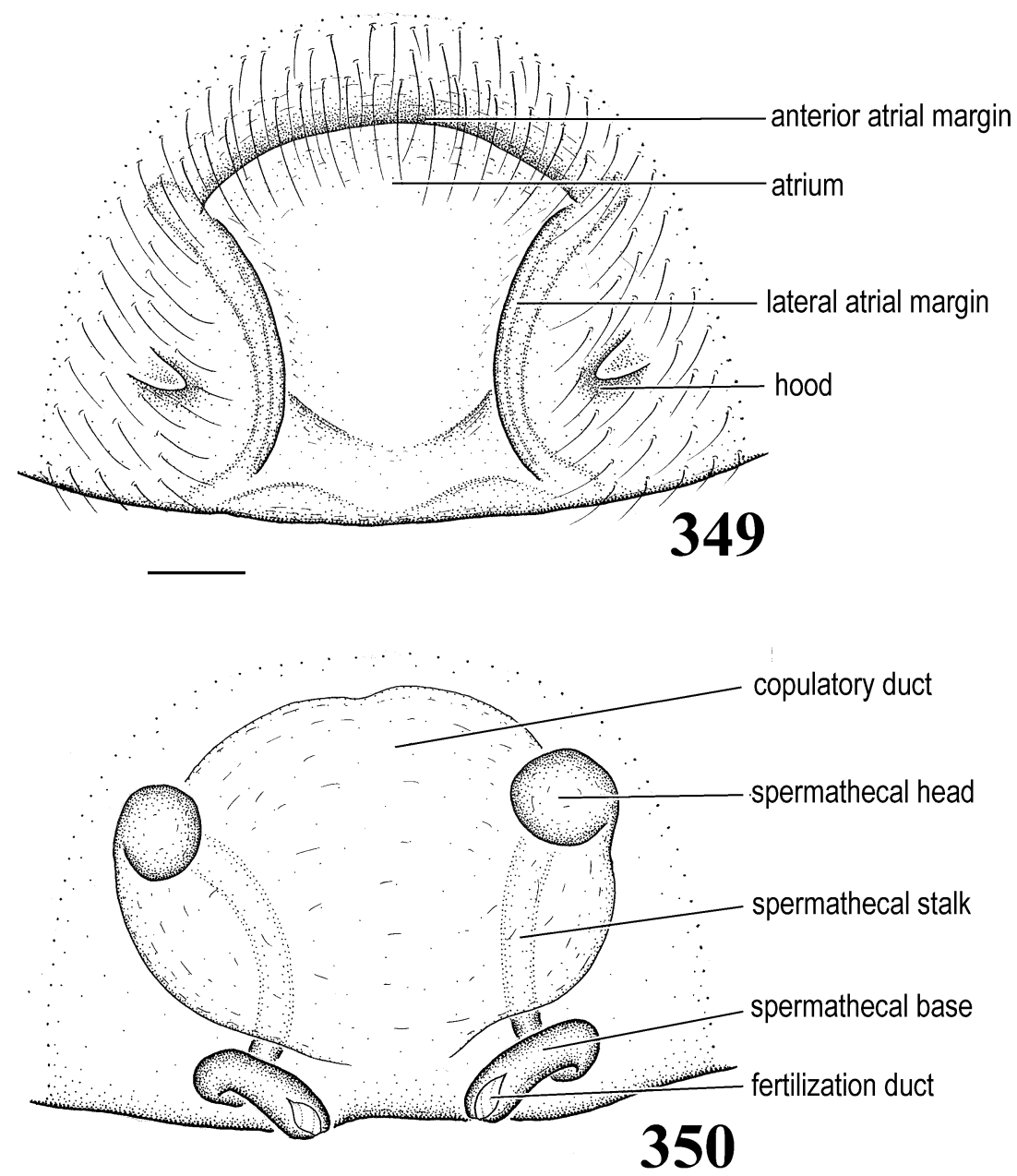

Figs. 349, 350. Robusticoelotes pichoni (Schenkel), female. 349. Epigynum. 350. Vulva.

from Haute Garonne, France, in MNHN, examined.

12. Paracoelotes spasskyi (Charitonov, 1946): male and female cotypes from Lagodeksk Natural Reserve, Khochal-Dag, Caucasus, Georgia, in PSU, examined.

13. Paracoelotes spinivulvus (Simon, 1881): female holotype and paratype from Peking, China, in MNHN, examined.

Coelotes qinlingensis Peng, Gong and Kim, 1996, with female holotype, male and female paratypes from Louguantan, Shaanxi, China, in HBI, examined, is placed as a junior synonym of $P$. spinivulvus (Simon) because of the identical male and female genitalic morphology. NEW SYNONYMY.

Cedicus mollendorffi Karsch, 1881, with 3 female syntypes from Peking, China, in ZMB, examined, is placed as a junior synonym of $P$. spinivulvus (Simon) because of the identical female genitalic morphology. NEW SYNONYMY.

Coelotes caraftensis Kishida, 1924, with types from Sakhalin, Russia, depository unknown, not examined, is placed as a junior synonym of $P$. spinivulvus (Simon). Judged by the illustrations, this species is identical with P. spinivulvus (Simon) in both male and female genitalia. NEW SYNONYMY.

Coelotes fascinatus Wang et al., 1990, with male and female paratypes from $\mathrm{Yu}$ zhong, Gansu, China, in HBI, examined, is placed as a junior synonym of $P$. spinivulvus (Simon) because of the identical genitalic morphology. NEW SYNONYMY. 


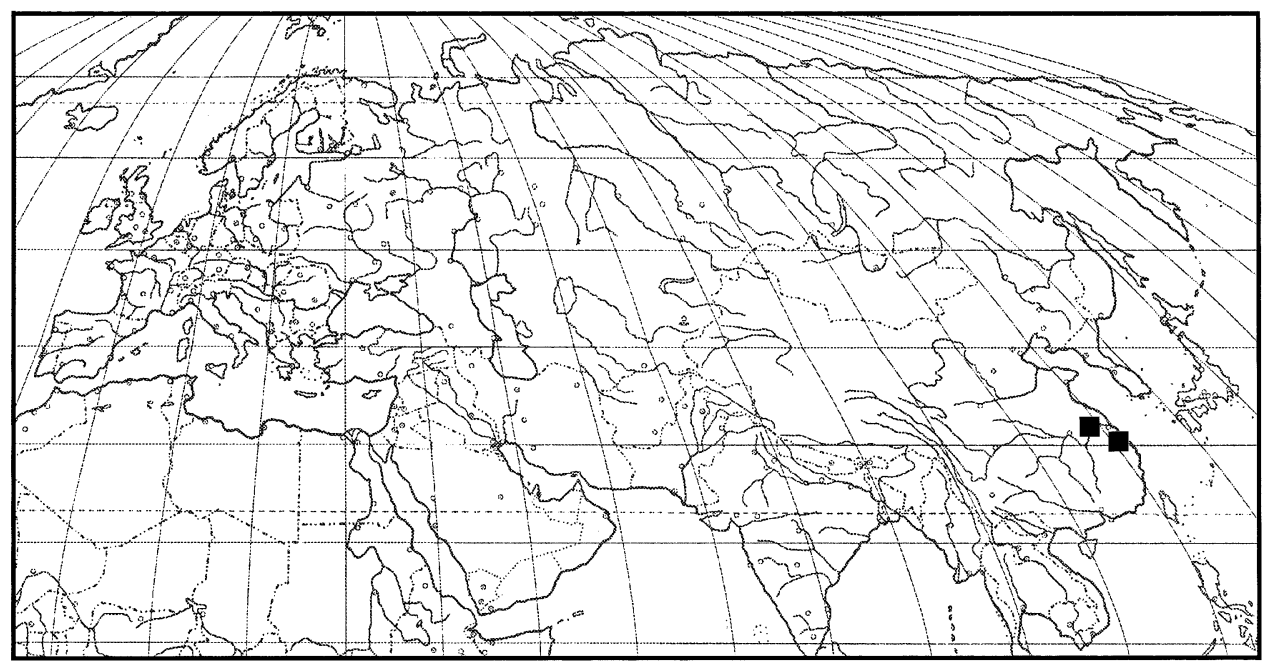

Map 15. Records of Robusticoelotes.

14. Paracoelotes taishanensis (Wang et al., 1990): male and female paratypes from Taishan, Shandong, China, in HBI, examined. NEW COMBINATION (transferred here from Coelotes).

Coelotes subluctuosus Zhu and Wang, 1994, with female holotype, male and female paratypes from Taishan, Shandong, China, in NBUMS, not examined, is placed as a junior synonym of $P$. taishanensis. Judged by the illustrations and the locality, this species is identical with $P$. taishanensis in male and female genitalia. NEW SYNONYMY.

15. Paracoelotes taiwanensis Wang and



Figs. 351, 352. Robusticoelotes pichoni (Schenkel), male palp. 351. Prolateral view. 352. Ventral view. 


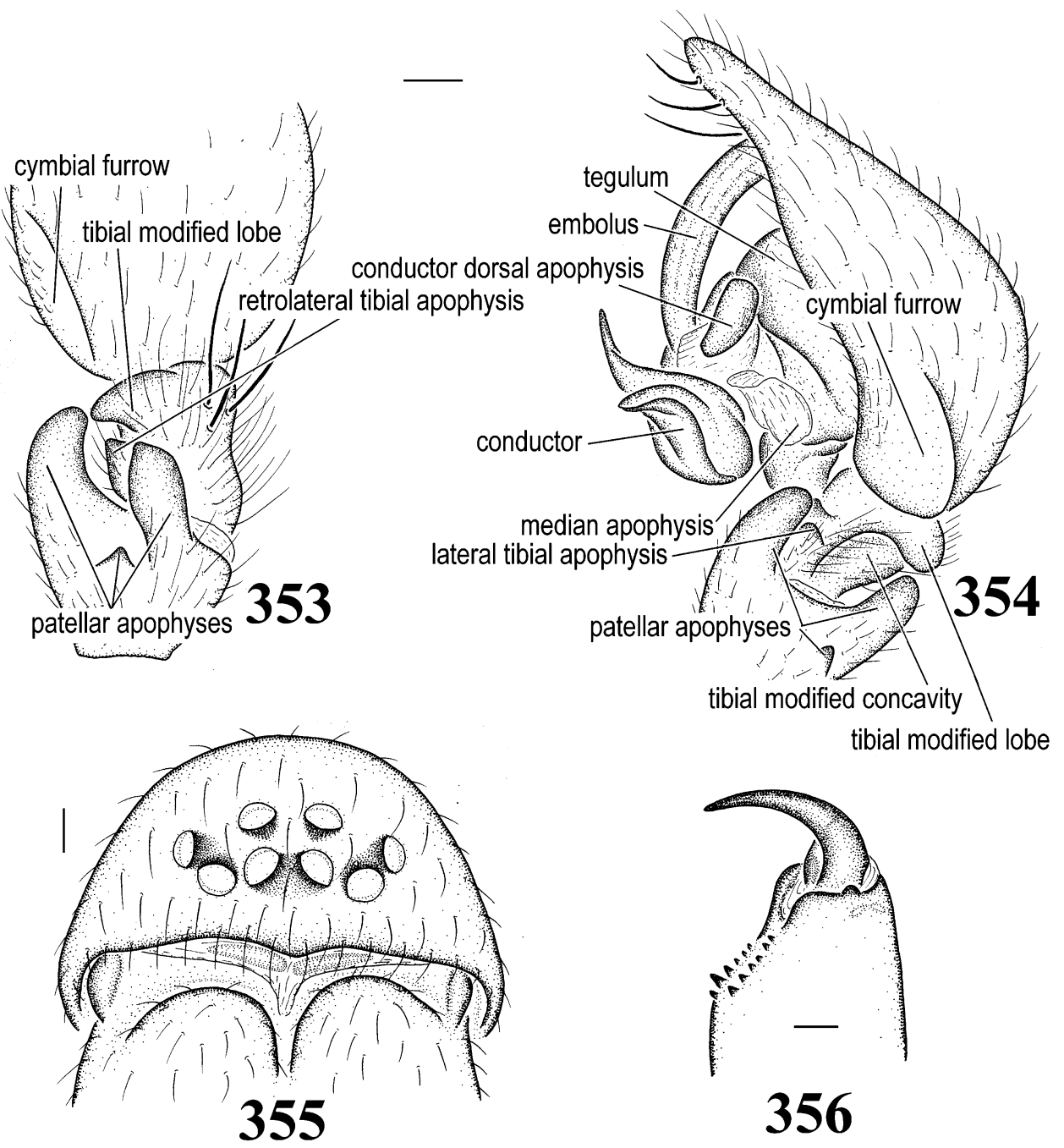

Figs. 353-356. Robusticoelotes pichoni (Schenkel), male palp. 353. dorsal view. 354. Retrolateral view. 355. Female, carapace, front view. 356. Female, chelicera, ventral view.

Ono, 1998: male holotype and female paratype from Tatachia, Nantou Hsien, Taiwan, in NSMT, examined.

16. Paracoelotes tianchiensis (Wang et al., 1990): male and female paratypes from Mt. Tianshan, Xinjiang, China, in HBI, examined. NEW COMBINATION (transferred here from Coelotes).

\section{Paracoelotes armeniacus (Brignoli) Figures 323-335}

Coelotes armeniacus Brignoli, 1978b: 527, figs. 117-123 (male holotype, male and female par- atypes from Zigana gecidi, Gumushane, Turkey, in MCV and MNHG, examined).

Paracoelotes armeniacus: Brignoli, 1982: 347.

Diagnosis: Females are similar to those of $P$. major but can be distinguished by the long spermathecal heads (fig. 324). Males resemble those of $P$. garibaldii but can be recognized by the relatively large patellar apophysis and the less extended RTA (figs. 325-327).

DESCRIPTION: Total length about 8.0014.0. From front, anterior eye row slightly procurved, posterior row procurved; eye sizes and arrangements: AME small, about 

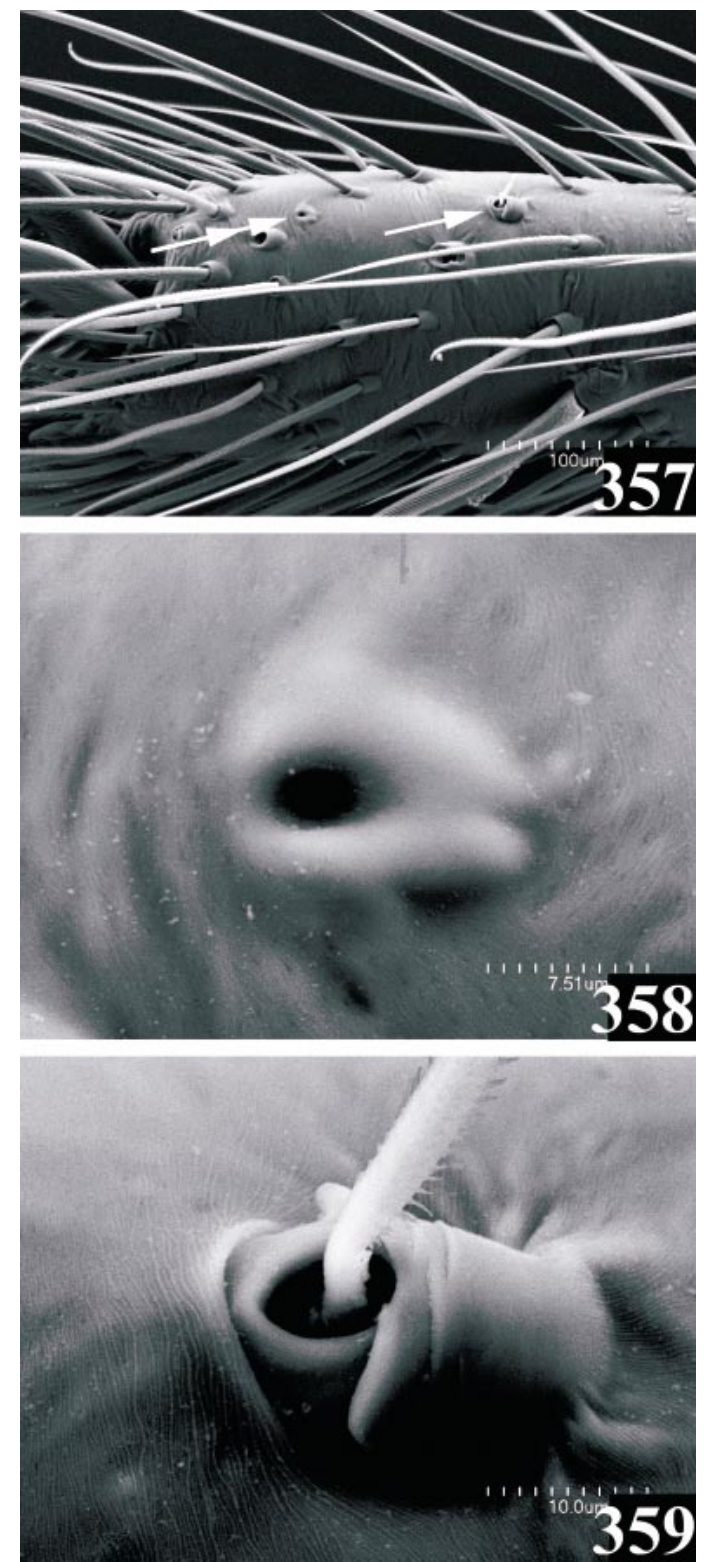

Figs. 357-359. Robusticoelotes pichoni (Schenkel), female. 357. Tarsus, showing position of tarsal organ (double arrows) and the distalmost trichobothrium (single arrow). 358. Tarsal organ. 359. Trichobothrium.

three-fourths ALE size, slightly smaller then PME, ALE largest, subequal or slightly bigger than PLE; AME may be separated by roughly their diameter or slightly less, AMEALE about AME diameter, ALE-PLE close together, PME-PLE about 1.5 PLE diameter, PME-PME slightly longer than PME diameter. Clypeal height about twice AME diameter or slightly less, covered with long, strong setae; chilum divided, hairless, long (fig. 328). Chelicerae with three promarginal, three retromarginal teeth (fig. 329).

Tarsal organ situated close to distal end of tarsus, slightly anterior of distalmost trichobothrium (figs. 330-332). Only female spinnerets examined: apex of ALS with 2 major ampullate gland spigots (MAP), at least 29 piriform gland spigots; PMS with 1 or $2 \mathrm{mi}-$ nor ampullate gland spigots (mAP), about 40 aciniform gland spigots, at least 4 cylindrical gland spigots (CY); PLS with about 40 aciniform gland spigots, 2 cylindrical gland spigots observed (figs. 333-335).

Epigynum with epigynal teeth situated anterolaterally, long; atrium large, with anterior margin extended posteriorly; copulatory ducts broad, membranous; spermathecal heads large, anteriorly situated; spermathecae longitudinally elongated, convoluted, widely separated, with indistinct bases and stalks (figs. 323, 324). Male palp with one small patellar apophysis; RTA with distal end blunt, slightly extended beyond tibia; lateral tibial apophysis small; cymbial furrow moderately long; conductor long, spiral with one apparent loop; conductor dorsal apophysis absent; conductor lamella moderately developed; embolus prolateral in origin, moderately long, linear; median apophysis spoonlike, with strongly extended distal end (figs. 325-327).

MATERIAL EXAMINED: TURKEY: Gumushane: Zigana gecidi, 2100 m, July 12, 1971, male holotype and 6 female paratypes (P. Brignoli, MNHG), Zigana gecidi, $2025 \mathrm{~m}$ alt., June 12, 1968, 3 female paratypes (P. Brignoli, MNHG); Zigana gecidi, $2100 \mathrm{~m}$, July 12, 1971, 2 male and 7 female paratypes (G. Osella, MCV); Soganli gecidi, $2400 \mathrm{~m}$, July 11, 1971, 15 females (G. Osella, MCV). Artvin: Savsat, 2450 m, July 6, 1971, 9 females (Vigna, MCV, Coll. Brignoli).

DistRIBUTION: Turkey (Gumushane, Artvin).

\section{PLATOCOELOTES, NEW GENUS}

TyPe SPecies: Coelotes impletus Peng and Wang, 1997.

ETYMOLOGY: This generic name is de- 


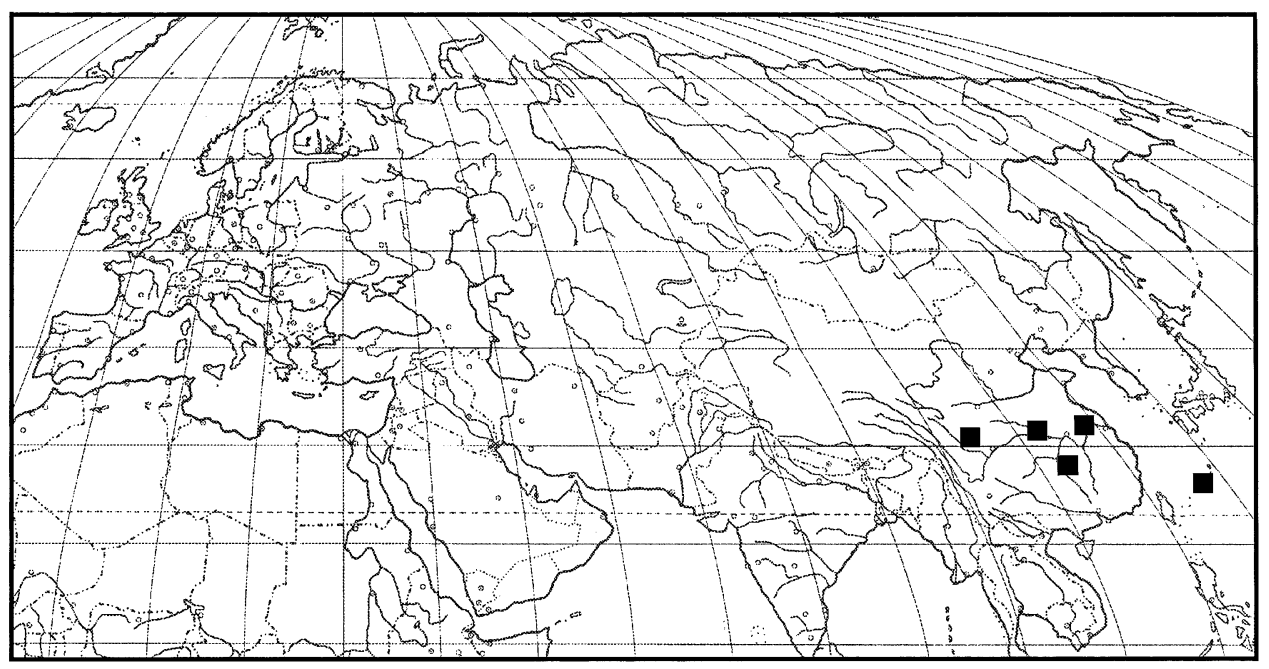

Map 16. Records of Spiricoelotes.

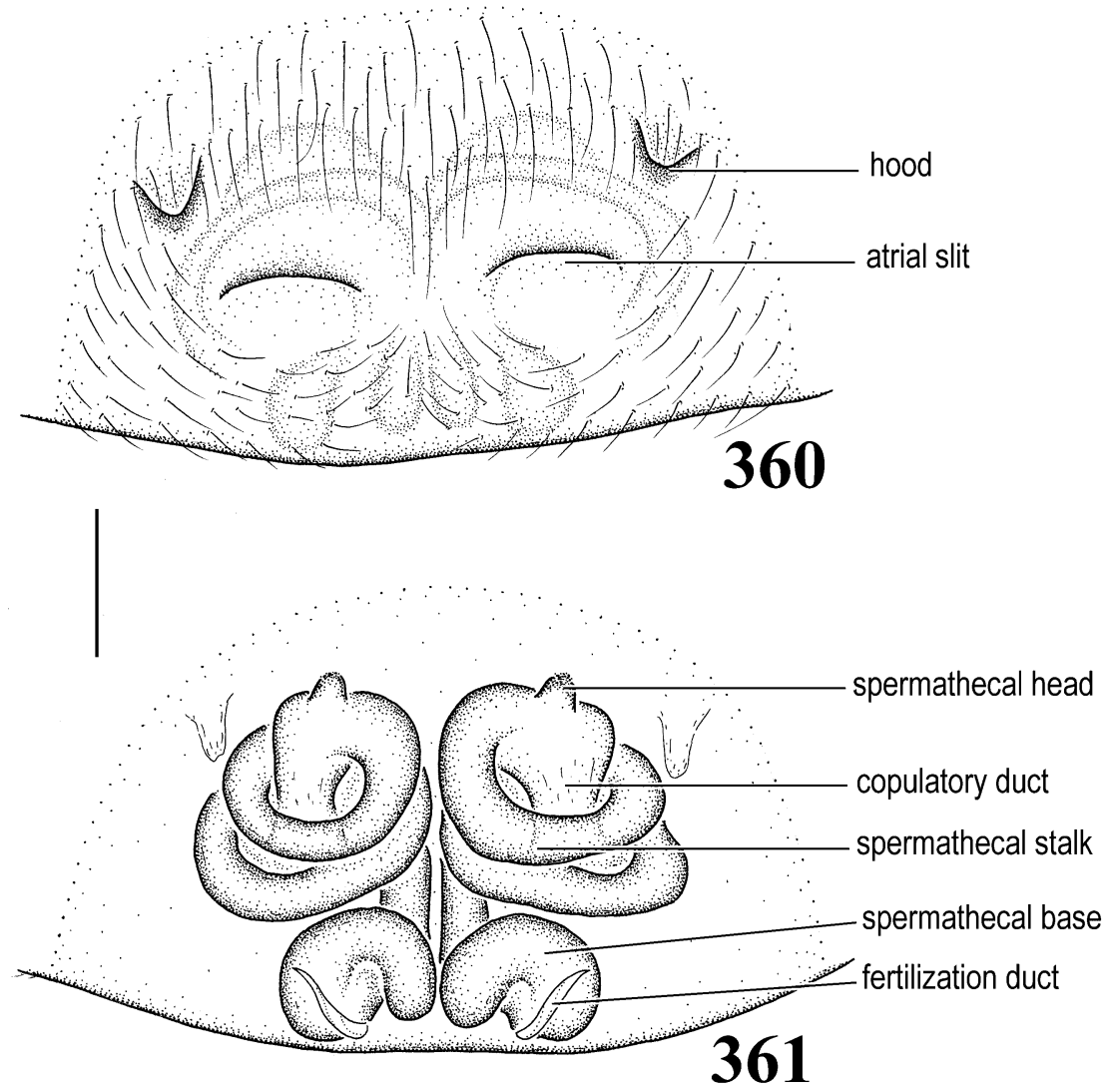

Figs. 360, 361. Spiricoelotes zonatus (Peng and Wang), female. 360. Epigynum. 361. Vulva. 

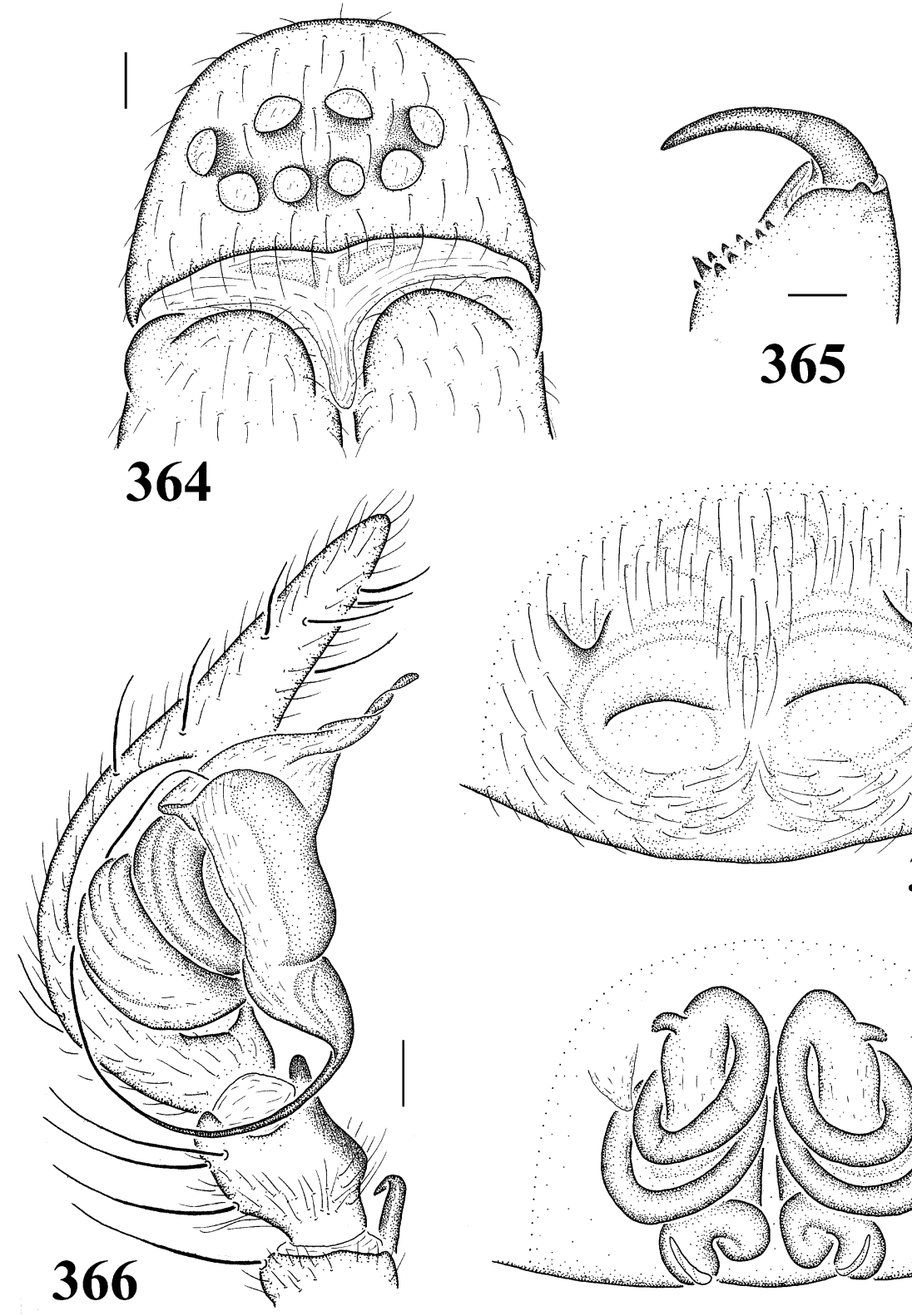

\section{5}


Figs. 362-366. Spiricoelotes zonatus (Peng and Wang). 362. Female, epigynum. 363. Female, vulva. 364. Female, carapace, front view. 365. Female, chelicera, ventral view. 366. Male palp, prolateral view.

rived from its similarity to Coelotes and to its broad epigynum, and is masculine in gender.

DiAgnOSIS: Females can be easily recognized by the absence of epigynal teeth, the presence of an anteriorly situated epigynal cavity, the deep, posteriorly situated epigynal hoods, and the long, strongly convoluted spermathecae (figs. 336, 337), and males by the presence of two patellar apophyses (one strongly reduced in some specimens), the elongated cymbial furrow, the presence of a conductor posterior apophysis, a long embolus, and the absence of a median apophysis 


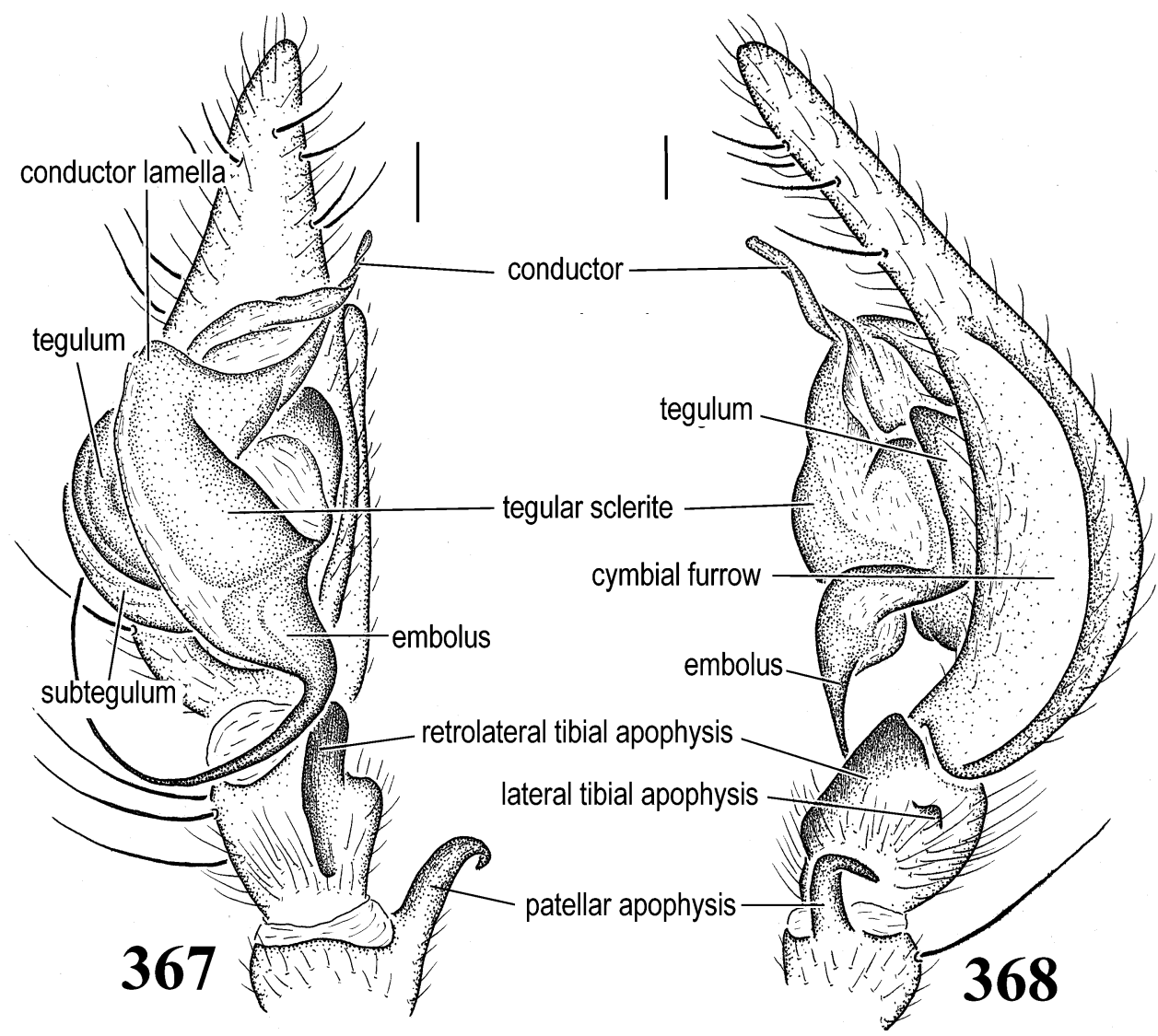

Figs. 367, 368. Spiricoelotes zonatus (Peng and Wang), male palp. 367. Ventral view. 368. Retrolateral view.

(figs. 338-340). Both sexes have 3 promarginal and 2 retromarginal cheliceral teeth (fig. 342).

DESCRIPTION: See description of type species (below).

DisTRIBUTION: China (map 14).

COMPOSITION: Four species are included in this genus.

1. Platocoelotes impletus (Peng and Wang, 1997): female holotype, male and female paratypes from Zhangjiajie, Hunan, China, in HBI, examined. NEW COMBINATION (transferred here from Coelotes).

2. Platocoelotes icohamatoides (Peng and Wang, 1997): female paratype from Naer Mt., Fenghuang, Hunan, China, in HBI, examined. NEW COMBINATION (transferred here from Coelotes).

3. Platocoelotes icohamatus (Zhu and
Wang, 1991): female holotype, male and female paratypes from Sichuan, China, in NBUMS, not examined. NEW COMBINATION (transferred here from Coelotes).

4. Platocoelotes lichuanensis (Chen and Zhao, 1998): male holotype from Lichuan, Hubei, China, in HUW, examined. NEW COMBINATION (transferred here from Coelotes).

\section{Platocoelotes impletus (Peng and Wang) Figures 336-348}

Coelotes impletus Peng and Wang, 1997: 328, figs. 11-19 (female holotype, male and female paratypes from Zhangjiajie, Hunan, China, in HBI, examined). - Song et al., 1999: 375, figs. 220D, 220E, 222H, 223K.

DiAgNosis: Females are similar to those of $P$. icohamatoides but can be distinguished by 

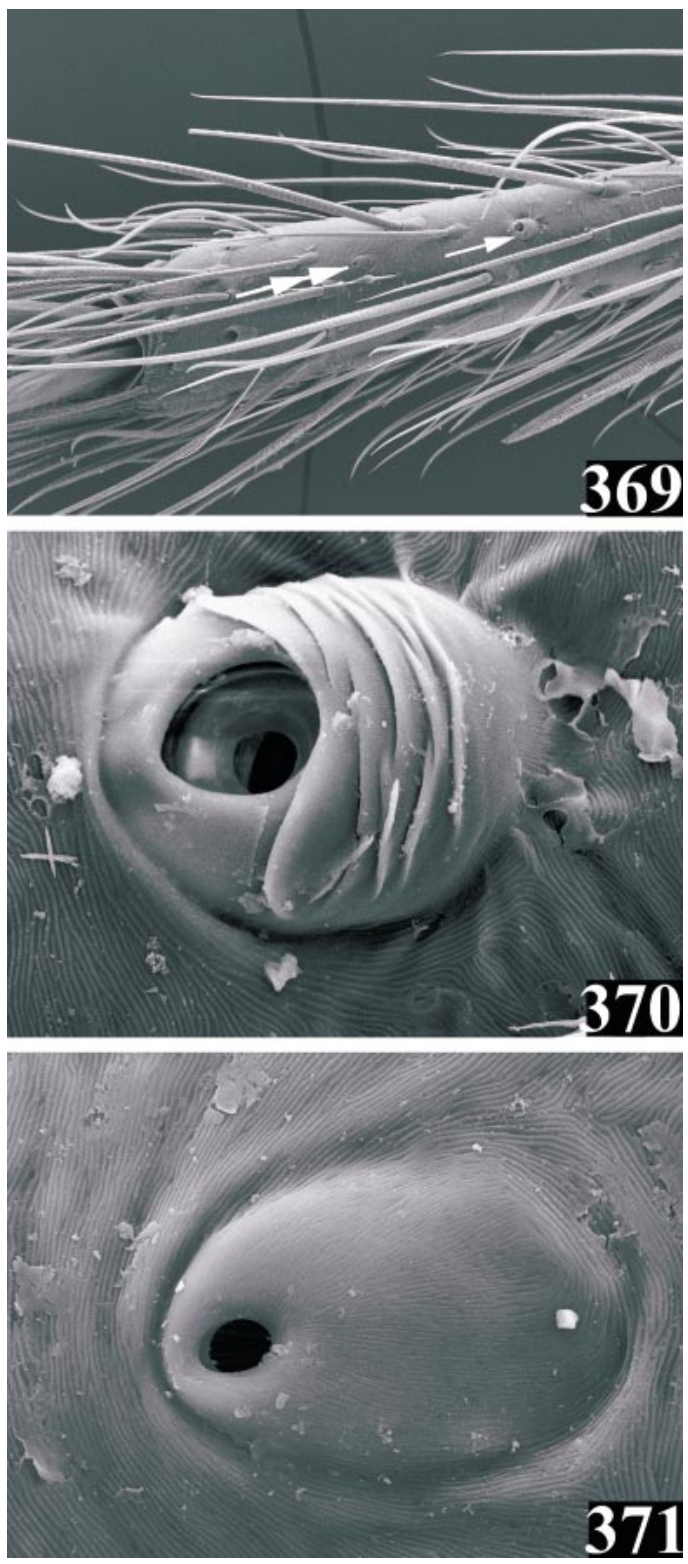

Figs. 369-371. Spiricoelotes zonatus (Peng and Wang), female. 369. Tarsus, showing position of tarsal organ (double arrows) and distalmost trichobothrium (single arrow). 370. Trichobothrium. 371. Tarsal organ.

the different shape of epigynum and the long spermathecal stalks (figs. 336, 337). Males can be distinguished from those of P. lichuanensis only by the size of the conductor apex apophyses (figs. 338-340).

DESCRIPTION: Total length 5.00-12.0.
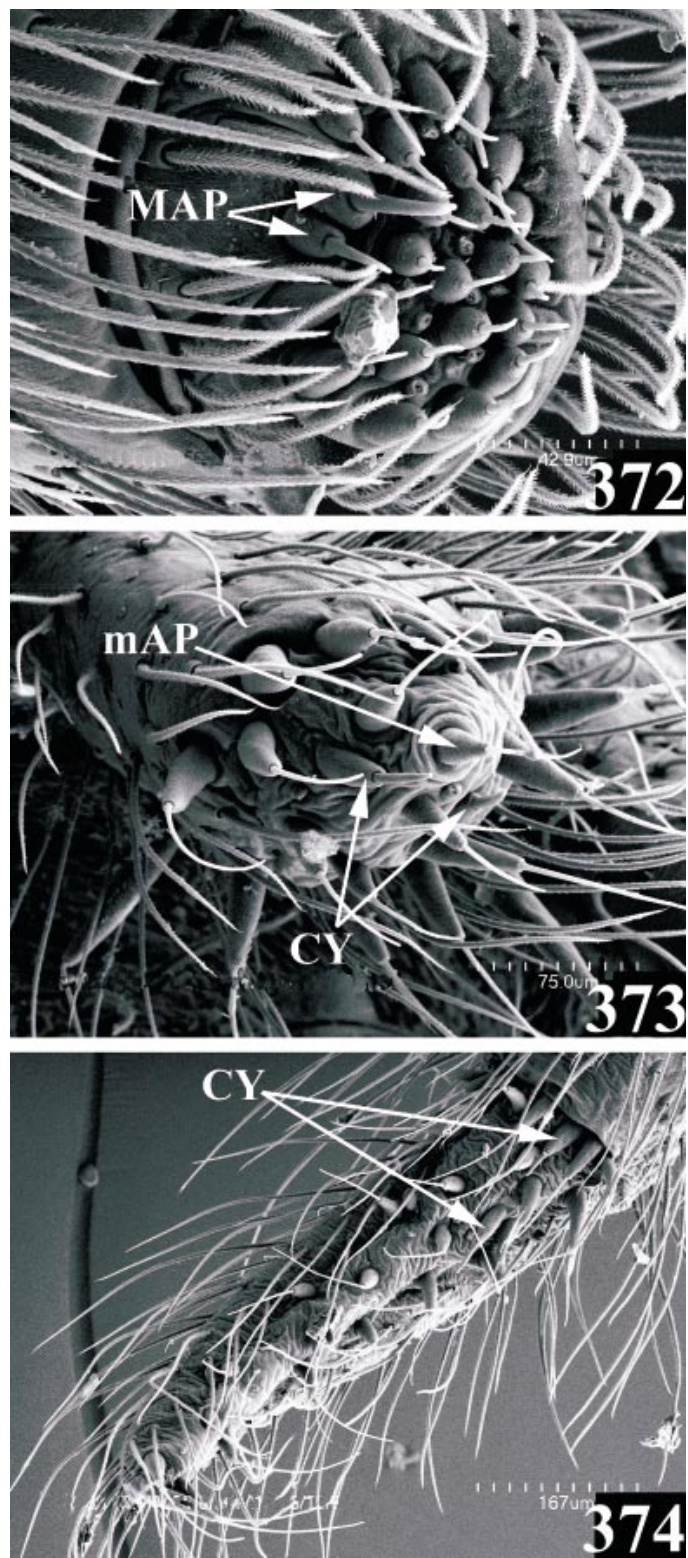

Figs. 372-374. Spiricoelotes zonatus (Peng and Wang), female spinnerets, ventral view, right. 372. ALS. 373. PMS. 374. PLS. Abbreviations are spelled out on p. 6 .

From front, anterior eye row slightly procurved, posterior row procurved; eye sizes and arrangements: AME slightly smaller than ALE, ALE subequal to PLE and PME; AME separated by roughly their radius, AME-ALE and ALE-PLE close together, PME-PLE 


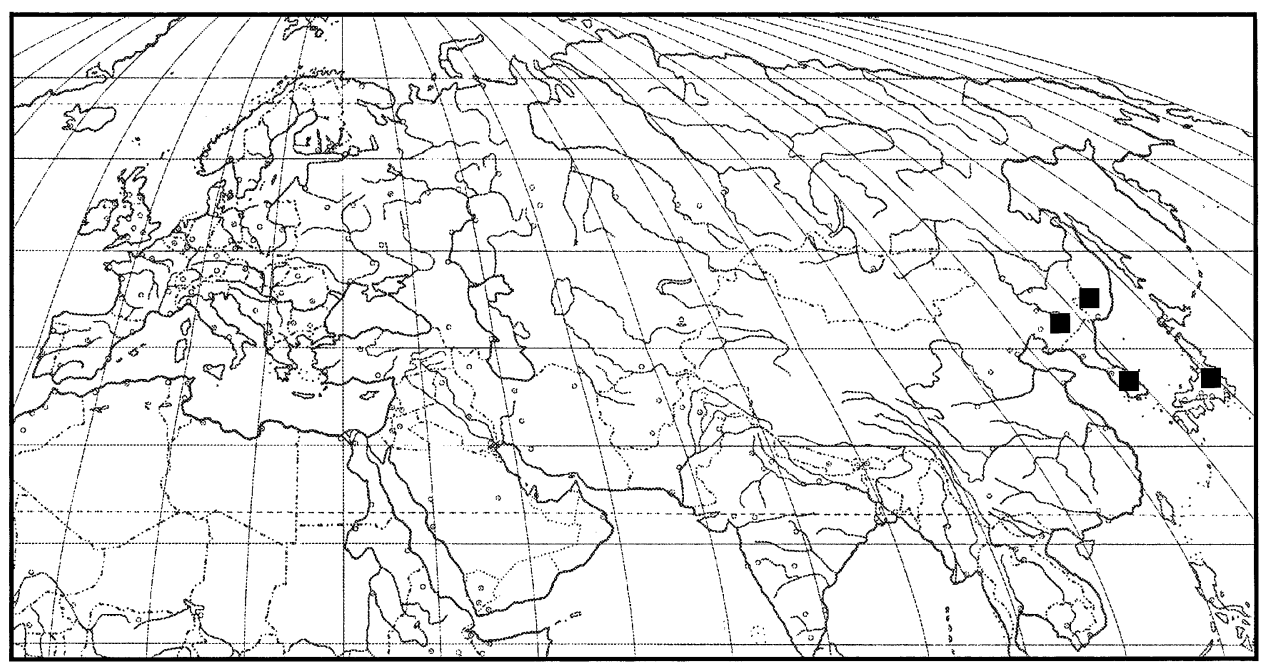

Map 17. Records of Tegecoelotes.

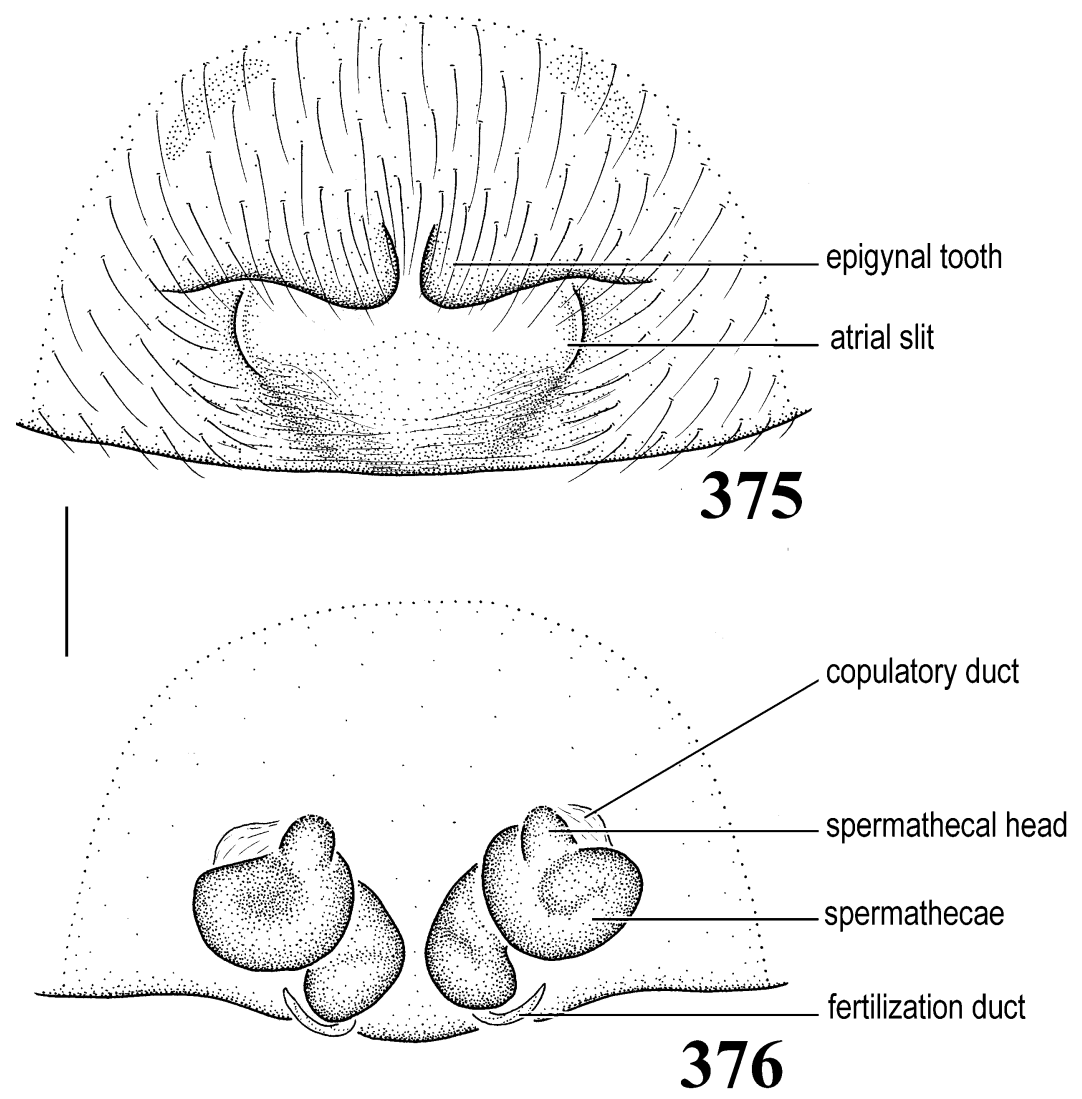

Figs. 375, 376. Tegecoelotes bicaudatus (Paik), female. 375. Epigynum. 376. Vulva. 




Figs. 377, 378. Tegecoelotes bicaudatus (Paik), male palp. 377. Prolateral view. 378. Retrolateral view.

about 1.5 PME diameter, PME-PME separated by roughly their radius. Clypeal height about twice AME diameter or slightly less; chilum divided (fig. 341). Chelicerae with three promarginal and two retromarginal teeth (fig. 342). Labium about as wide as long. Length of female 1 st leg patella + tibia longer than carapace length.

Tarsal organ not seen under SEM in examined specimens (figs. 343, 344). Further examination of other species also failed to locate tarsal organ (fig. 345). Female spinnerets examined: apex of ALS with 2 major ampullate gland spigots (MAP), 64 piriform gland spigots; PMS with at least 27 aciniform gland spigots, 2 cylindrical gland spig- ots (CY), and 1 or 2 minor ampullate gland spigots (mAP); PLS with at least 44 aciniform gland spigots, cylindrical gland spigots not clear in examined specimen (figs. 346348).

Epigynum without epigynal teeth; hoods situated posteriorly near epigastric margin; atrium longitudinally elongated, shallow; epigynum with anteriorly situated, shallow cavity; copulatory ducts short; spermathecal heads small; spermathecae with stalks strongly elongated, highly convoluted; spermathecal bases small (figs. 336, 337). Male palp with two small patellar apophyses; RTA long, occupying almost tibial length, with distal end strongly extended beyond tibia; 


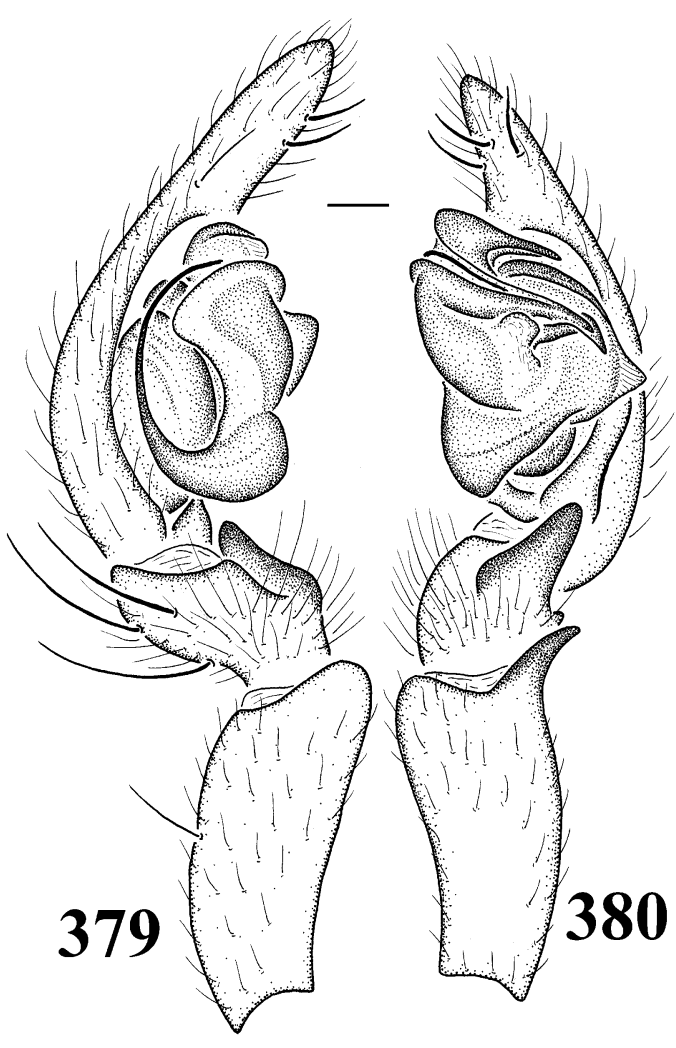

Figs. 379, 380. Tegecoelotes bicaudatus (Paik), male palp. 379. Prolateral view. 380. Retrolateral view.

lateral tibial apophysis small; cymbial furrow long, with distal end strongly extended, slightly beyond cymbium; conductor broad, with long posteriorly extended apophysis and anteriorly extended finger-like apophyses; conductor dorsal apophysis broad; conductor lamella moderately developed; embolus posterior in origin and then curved prolaterally, long, linear; median apophysis absent (figs. 338-340).

MAterial EXAMined: CHINA: Hunan: Zhangjiajie, August 18-20, 1988, 2 male and 2 female types (J. F. Wang and X. J. Peng, HBI).

DisTRIBUTION: China (Hunan).

\section{ROBUSTICOELOTES, NEW GENUS}

TyPE SPECIES: Tegenaria pichoni Schenkel, 1963.

ETYMology: The generic name is derived
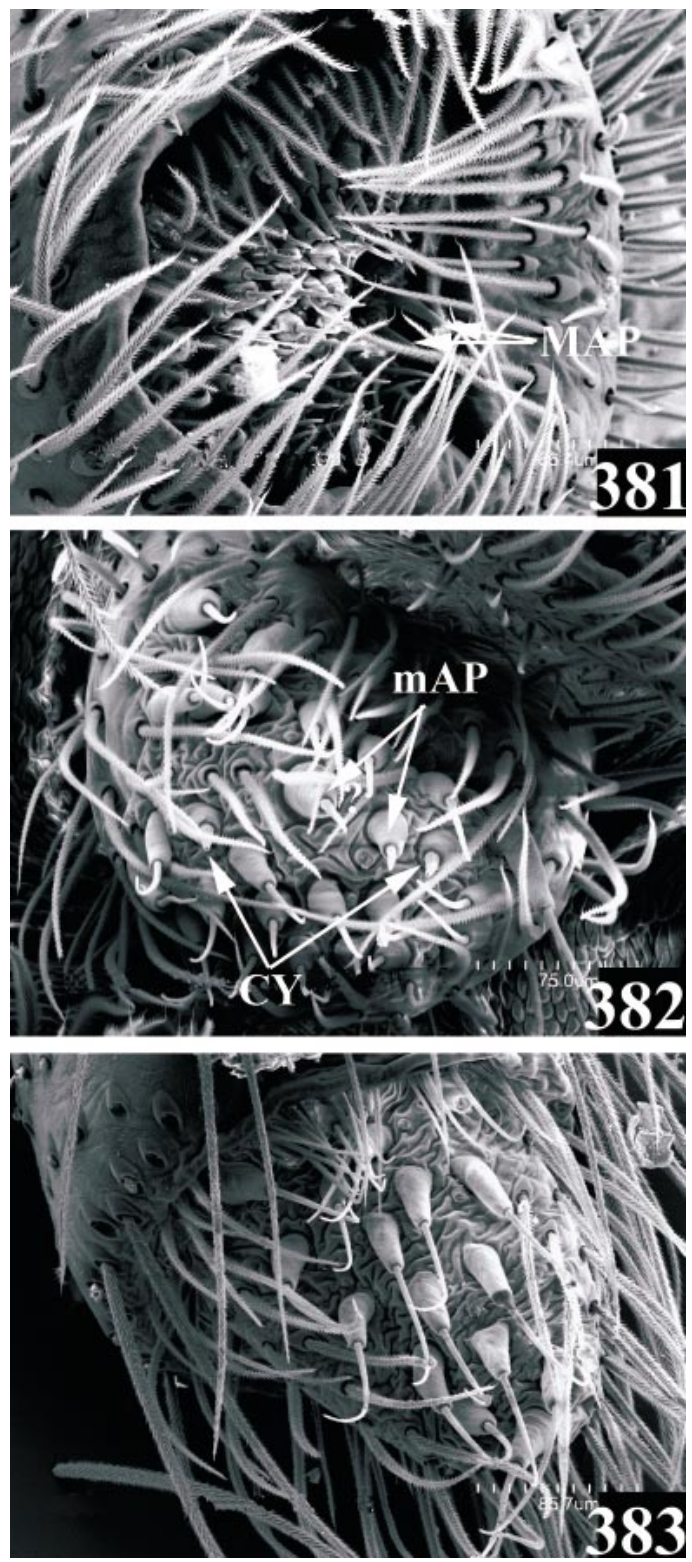

Figs. 381-383. Tegecoelotes bicaudatus (Paik), female spinnerets, ventral view. 381. ALS, left. 382. PMS, right. 383. PLS, right. Abbreviations are spelled out on p. 6.

from Coelotes and the strongly developed patellar apophyses, conductor, and embolus, and is masculine in gender.

DiAGNOSIS: Females resemble Ambanus in having a large epigynal atrium, broad copulatory ducts, and in lacking epigynal teeth, 

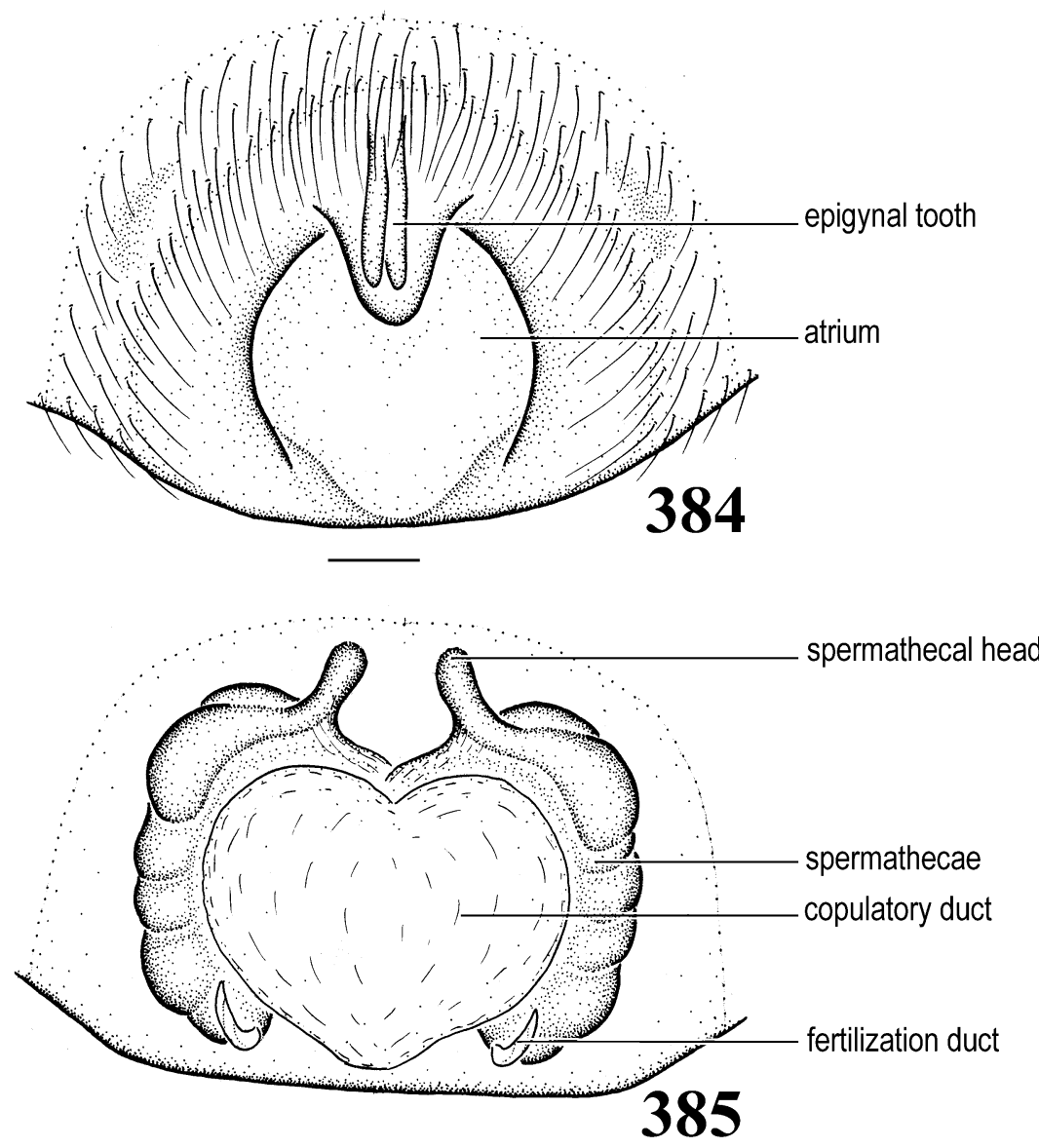

Figs. 384, 385. Tonsilla truculenta Wang and Yin, female. 384. Epigynum. 385. Vulva.

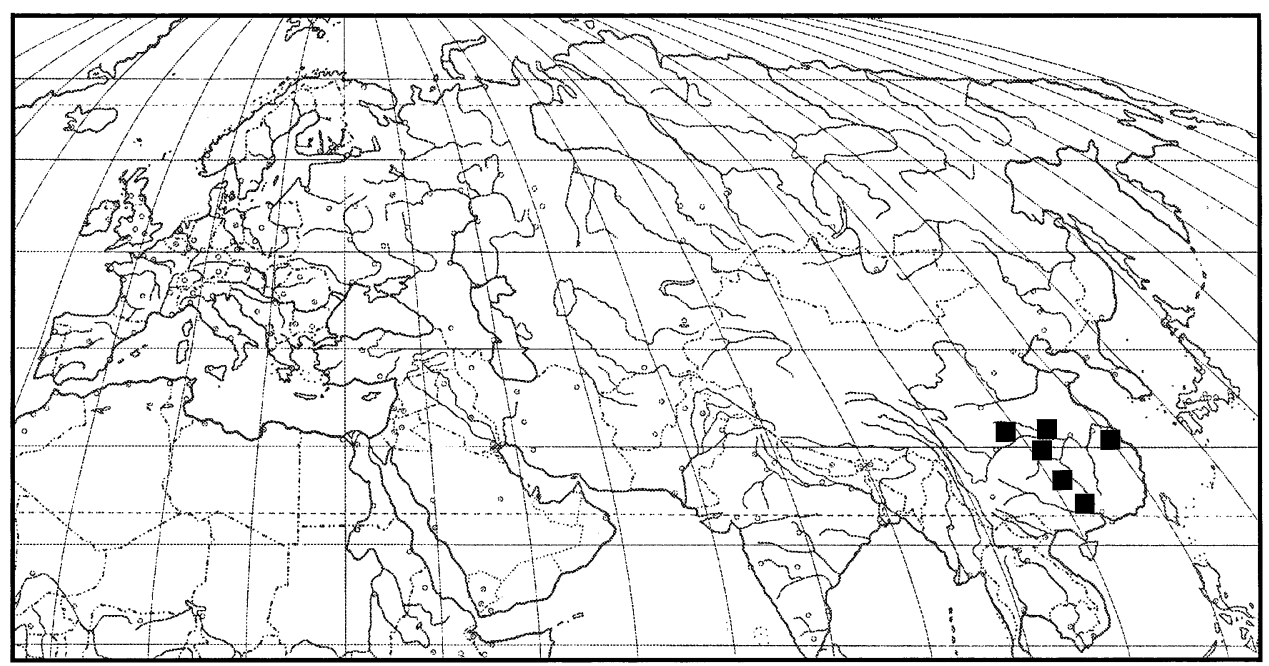

Map 18. Records of Tonsilla. 


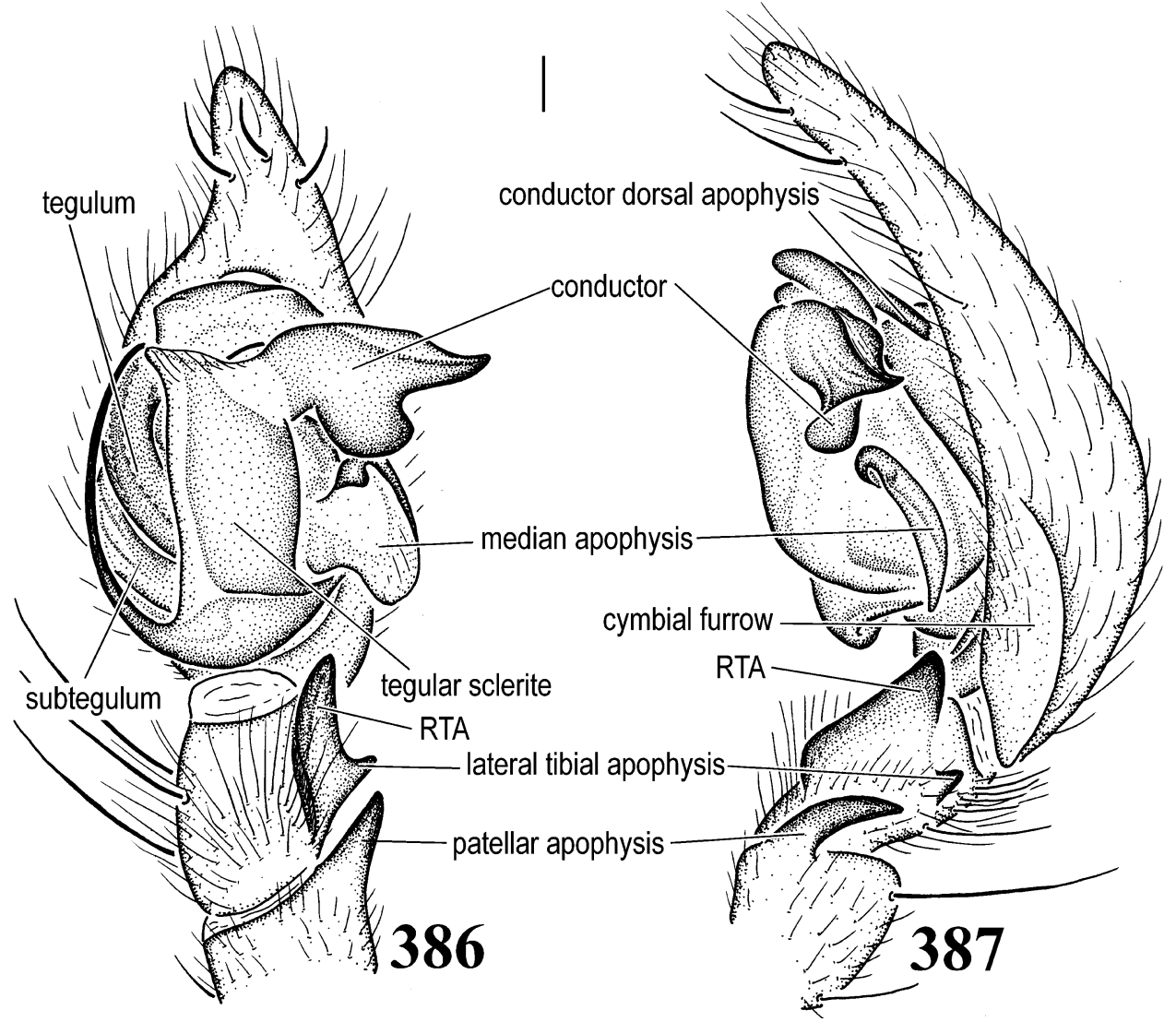

Figs. 386, $387 . \quad$ Tonsilla truculenta Wang and Yin, male palp. 386. Ventral view. 487. Retrolateral view.

but have long, anteriorly extended spermathecae (figs. 349, 350). Males can be easily recognized by the presence of three patellar apophyses and the strongly modified retrolateral surface of the palpal tibia (figs. 351354). Both sexes have six or seven promarginal and five or six retromarginal teeth (fig. 356), whereas most coelotines have three promarginal and less then four retromarginal teeth.

DESCRIPTION: See description of type species (below).

DisTRIBUTION: China (map 15).

COMPOSITION: Only $R$. pichoni.

Robusticoelotes pichoni (Schenkel), new combination

Figures 349-359

Tegenaria pichoni Schenkel, 1963: 277, fig. 157ad (1 male and 1 female type from Hangtscheou,
Tschekiang, China, in MNHN, examined). Chen and Zhang, 1991: 191, fig. 191; - Song et al., 1999: 360, figs. 209I, 209J, 209P, 209Q.

Diagnosis: This species can be easily recognized by lacking epigynal teeth, the broad atrium, the broad copulatory ducts, the large, anteriorly situated spermathecal heads, and the long, slender spermathecae of females (figs. 349,350 ), and males by having three patellar apophyses, the tibial modification, the strong, spiral, bifid conductor, the broad, membranous median apophysis, and the strong embolus (figs. 351-354).

DESCRIPTION: Total length 6.01-8.44. From front, anterior eye row slightly procurved, posterior row procurved; AME, ALE, PLE subequal or ALE slightly larger, PME slightly smaller; AME-AME about AME radius, AME-ALE and ALE-PLE close together, PME-PLE wider than their diameter, PME- 

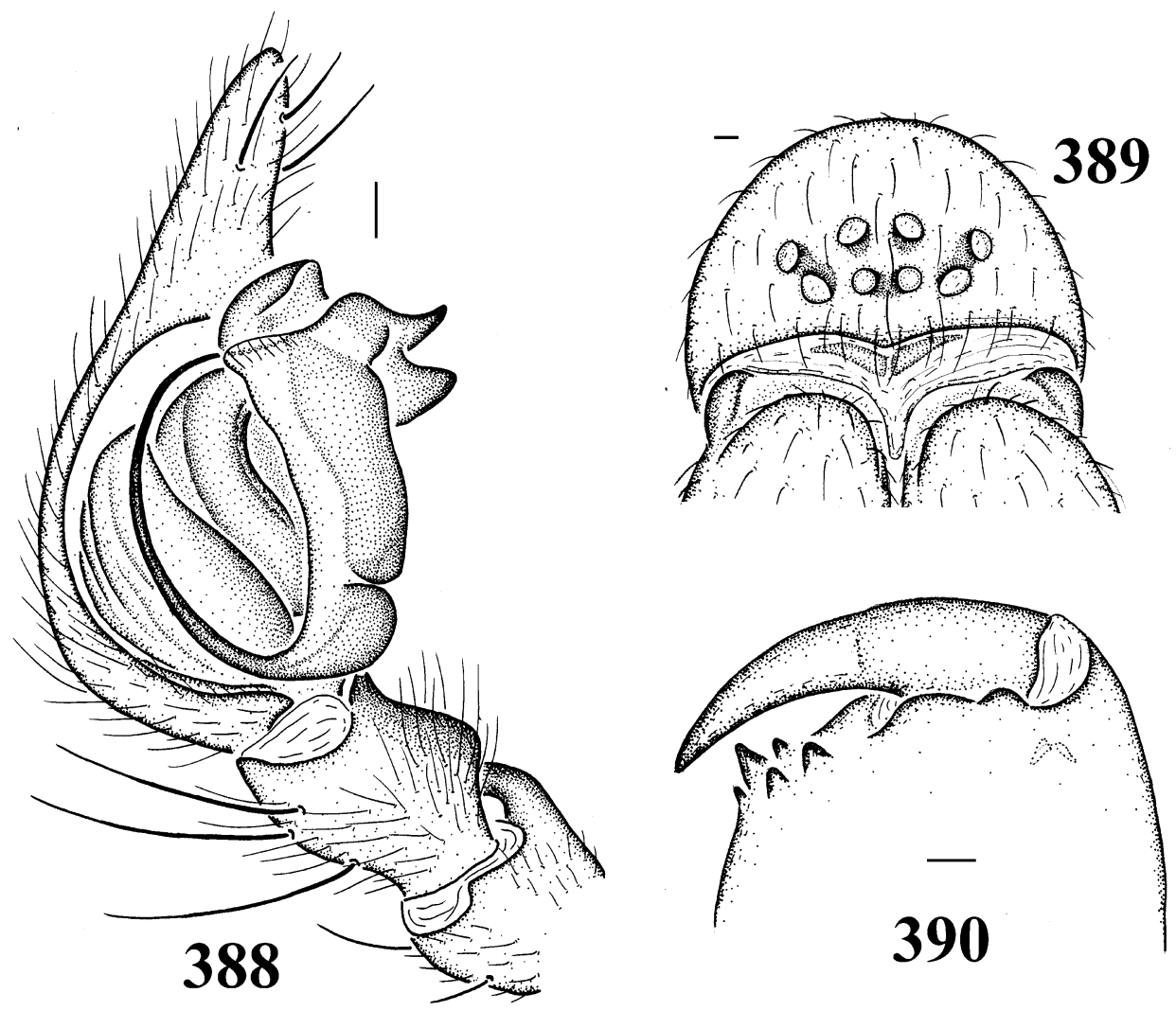

Figs. 388-390. Tonsilla truculenta Wang and Yin. 388. Male palp, prolateral view. 389. Carapace, front view. 390. Chelicera, ventral view.

PME shorter than their diameter. Clypeal height roughly twice AME diameter or slightly shorter; chilum divided, elongated (fig. 355). Chelicerae with six or seven promarginal and five or six retromarginal teeth (fig. 356). Labium slightly longer than wide. Length of female first leg patella + tibia shorter than carapace length.

Tarsal organ situated close to distal end of tarsus, anterior of distalmost trichobothrium (figs. 357-359). Spinnerets not examined.

Epigynum without teeth; atrium large, with apparent anterior and lateral atrial margins; lateral atrial margins convex toward each other; hoods posteriorly situated; copulatory duct broad; spermathecal heads large, situated anteriorly; spermathecae stalks long, slender, widely separated, not convoluted, covered by copulatory ducts in dorsal view; spermathecal bases more or less elongated, not convoluted (figs. 349, 350). Male palp with three patellar apophyses, two strong, one small; RTA short, with distal end not extended; lateral tibial apophysis present; lateral tibial surface strongly modified with lobes and concavity; cymbial furrow short; conductor strong, broad, spiral, with bifid apex; conductor lamella less developed; conductor dorsal apophysis short; median apophysis membranous, broad, not spoonlike; embolus basal in origin, strong, long (figs. 351-354).

Material Examined: CHINA: Zhejiang: Hangtscheou (Hangzhou), 1925, 1 male and 1 female type (MNHN, B-1979); Lin-an, October 19, 1974, 1 male (C. D. Zhu, NBUMS). Jiangsu: Nanking, April 1925, 1 female (C. Ping, AMNH).

Distribution: China (Zhejiang, Jiangsu).

\section{SPIRICOELOTES NEW GENUS}

Type Species: Coelotes zonatus Peng and Wang, 1997. 

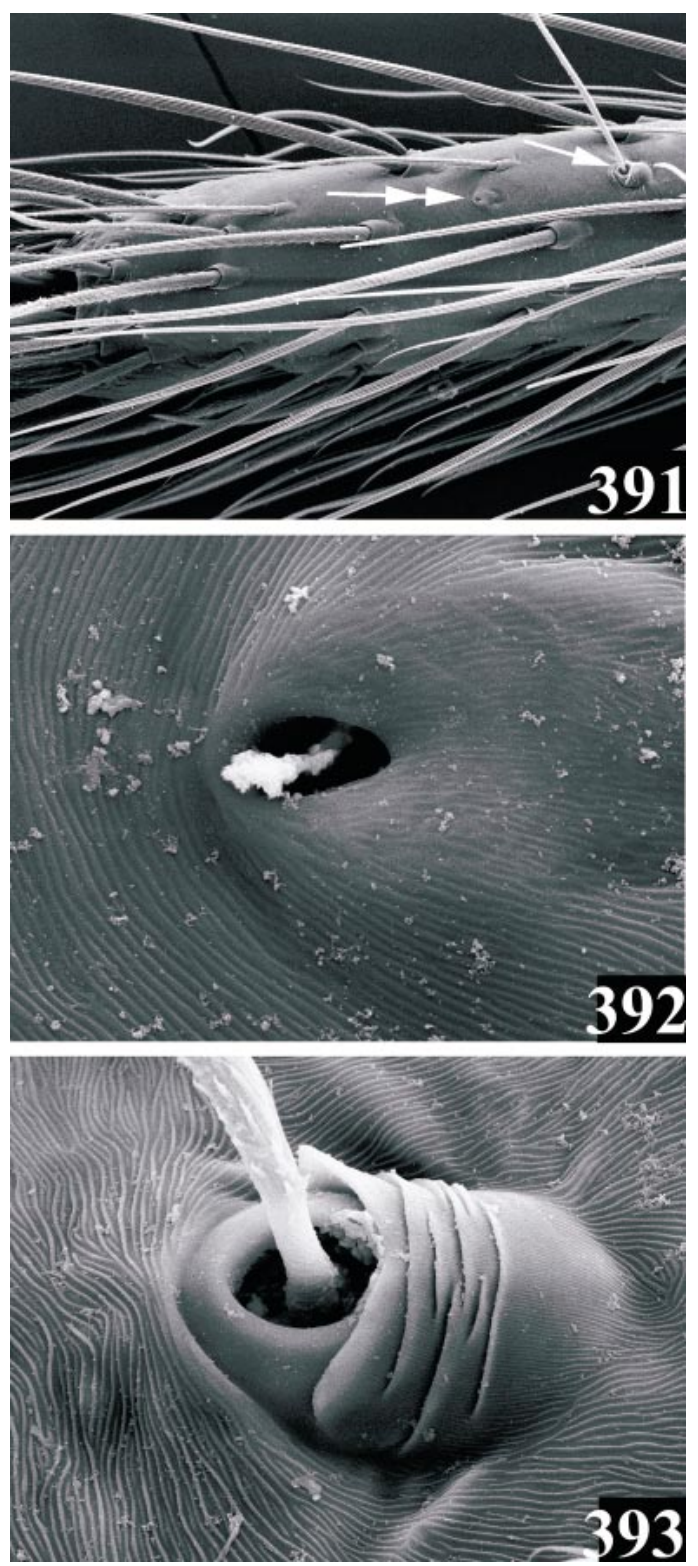

Figs. 391-393. Tonsilla truculenta Wang and Yin, female. 391. Tarsus, showing position of tarsal organ (double arrows) and the distalmost trichobothrium (single arrow). 392. Tarsal organ. 393. Trichobothrium.

ETYMology: The generic name is derived from Coelotes and the spiral conductor, the strongly spiral, convoluted spermathecae, and is masculine in gender.

DiAGNOSIS: Females can be easily recognized by the the long, strongly convoluted
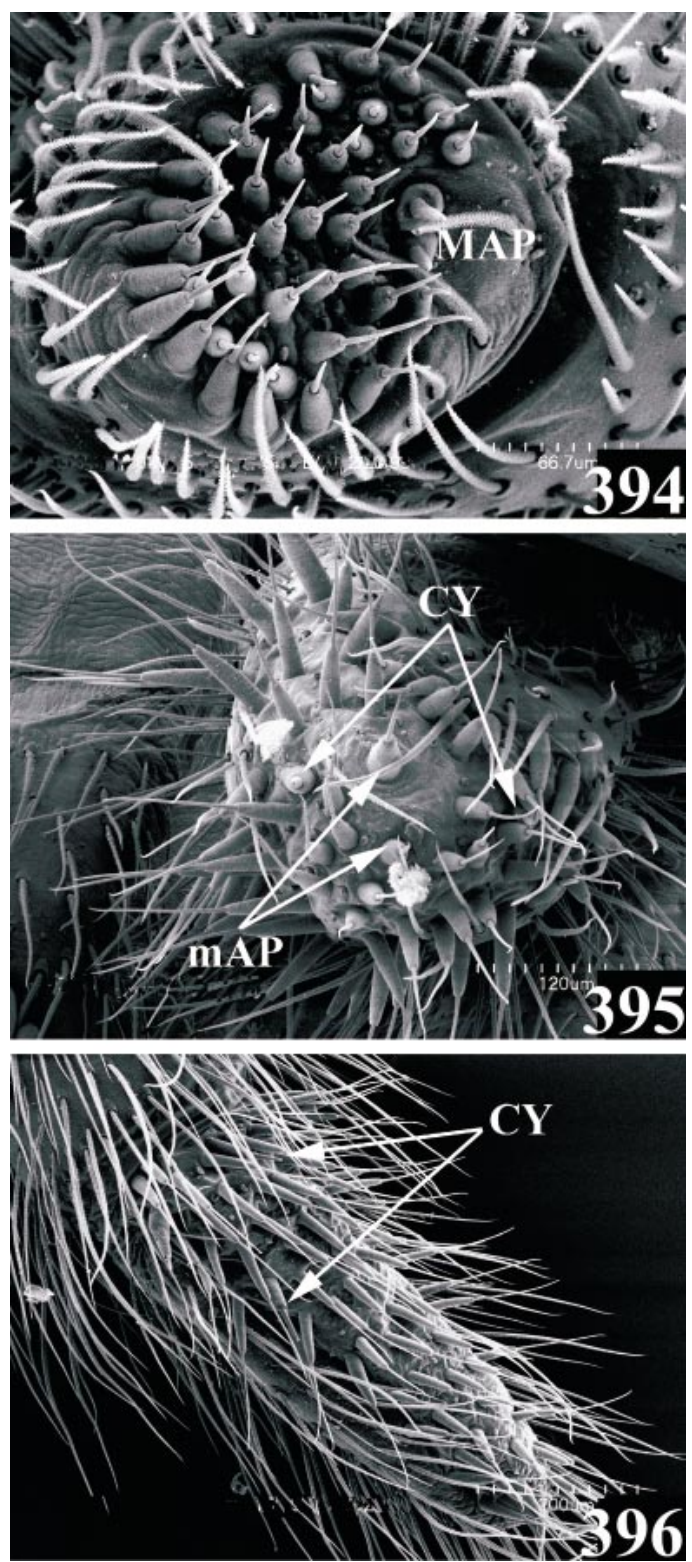

Figs. 394-396. Tonsilla truculenta Wang and Yin, female spinnerets, ventral view. 394. ALS, left. 395. PMS, right. 396. PLS, left. Abbreviations are spelled out on p. 6.

spermathecae and the absence of epigynal teeth (figs. 360, 362), males by the strongly curved patellar apophysis, the elongated cymbial furrow, the absence of a conductor dorsal apophysis, and the slender, anteriorly extended, spiral conductor (figs. 366-368). 

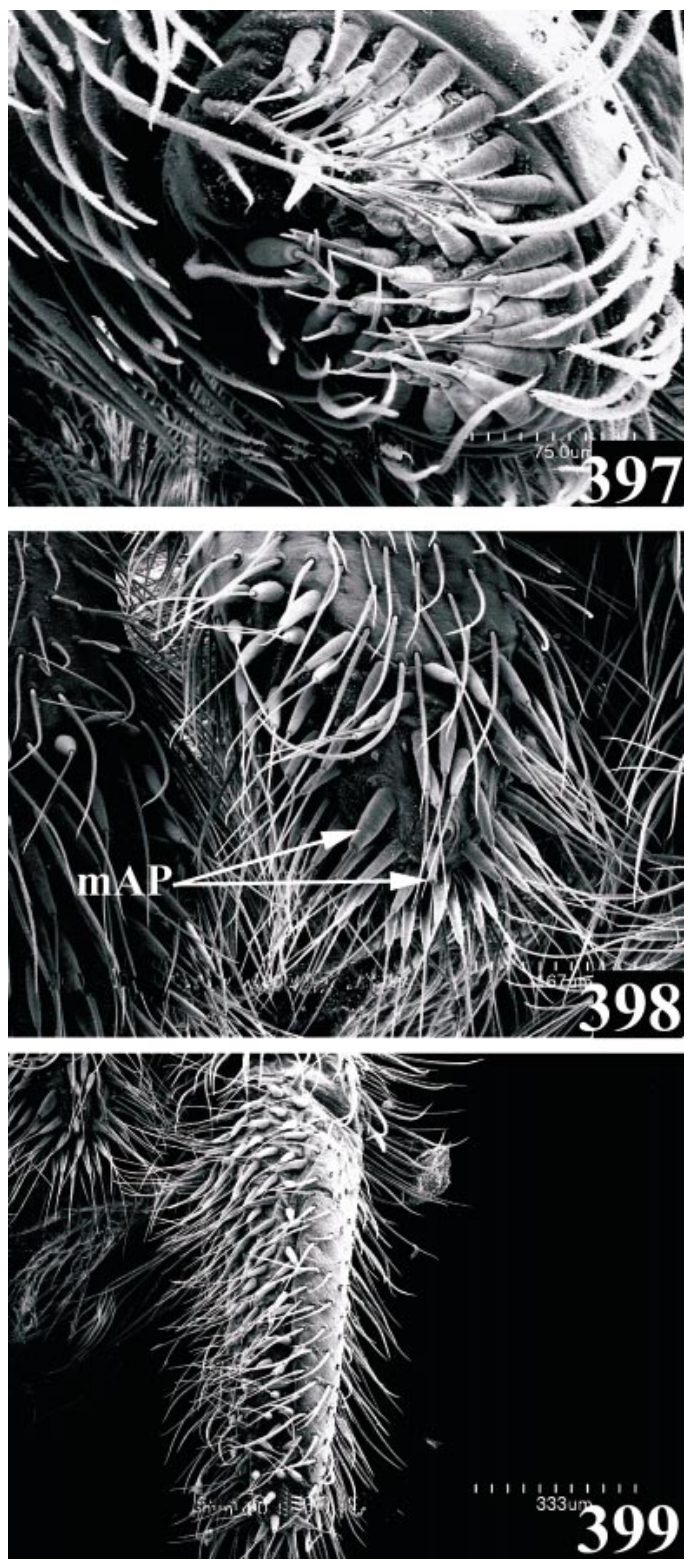

Figs. 397-399. Tonsilla truculenta Wang and Yin, male spinnerets, ventral view, right. 397. ALS. 398. PMS. 399. PLS. Abbreviations are spelled out on p. 6.

Both sexes have five teeth on both cheliceral margins (fig. 365).

DESCRIPTION: See description of type species (below).

DisTRIBUTION: China, Japan (map 16).

Composition: Two species are included in this genus.
1. Spiricoelotes urumensis (Shimojana, 1989): male holotype, male and female paratypes from Ishigaki City, Japan, in NSMT, not examined. NEW COMBINATION (transferred here from Coelotes).

2. Spiricoelotes zonatus (Peng and Wang, 1997): male and female paratypes from Changsha, Hunan, China, in HBI, examined. NEW COMBINATION (transferred here from Coelotes).

\section{Spiricoelotes zonatus (Peng and Wang) Figures 360-374}

Coelotes zonatus Peng and Wang, 1997: 331, figs. 32-36 (male and female paratypes from Changsha, Hunan, China, in HBI, examined). - Song et al., 1999: 376, figs. 220H, 220I, 222I, 223L. Coelotes laoyingensis Chen and Zhao, 1997: 89, figs. 5, 6 (female holotype and female paratype from Laoying, Wudangshan, Hubei, China, in HUW, examined). - Song et al., 1999: 388, figs. 226O, 226P, 227P, 229B. NEW SYNONYMY.

Diagnosis: This species can be distinguished from $S$. urumensis by the strong spermathecae of females (fig. 361) and the relatively short conductor of males (fig. 367).

DESCRIPTION: Total length 6.00-8.00. From front, anterior eye row slightly procurved, posterior row procurved; eye sizes and arrangements: AME smallest, slightly smaller than ALE, ALE, PLE and PME subequal; AME separated by roughly three-fourths their diameter, AME-ALE and ALE-PLE close together, PME-PLE separated by roughly three-fourths their diameter, PMEPME subequal to AME-AME or slightly longer. Clypeal height slightly longer than AME diameter; chilum divided (fig. 364). Chelicerae with five teeth on both margins (fig. 365). Labium wider than long. Length of female 1st leg patella + tibia longer than carapace length.

Tarsal organ situated anterior of most distal trichobothrium (figs. 369-371). Only female spinnerets examined: apex of ALS with 2 major ampullate gland spigots (MAP), about 23 piriform gland spigots; PMS with spigots with 1 or 2 minor ampullate gland spigots (mAP), about 20 aciniform gland spigots, 2 cylindrical gland spigots (CY); PLS with about 30 aciniform gland spigots, 2 cylindrical gland spigots (figs. 372-374). 




Map 19. Records of Urocoras.

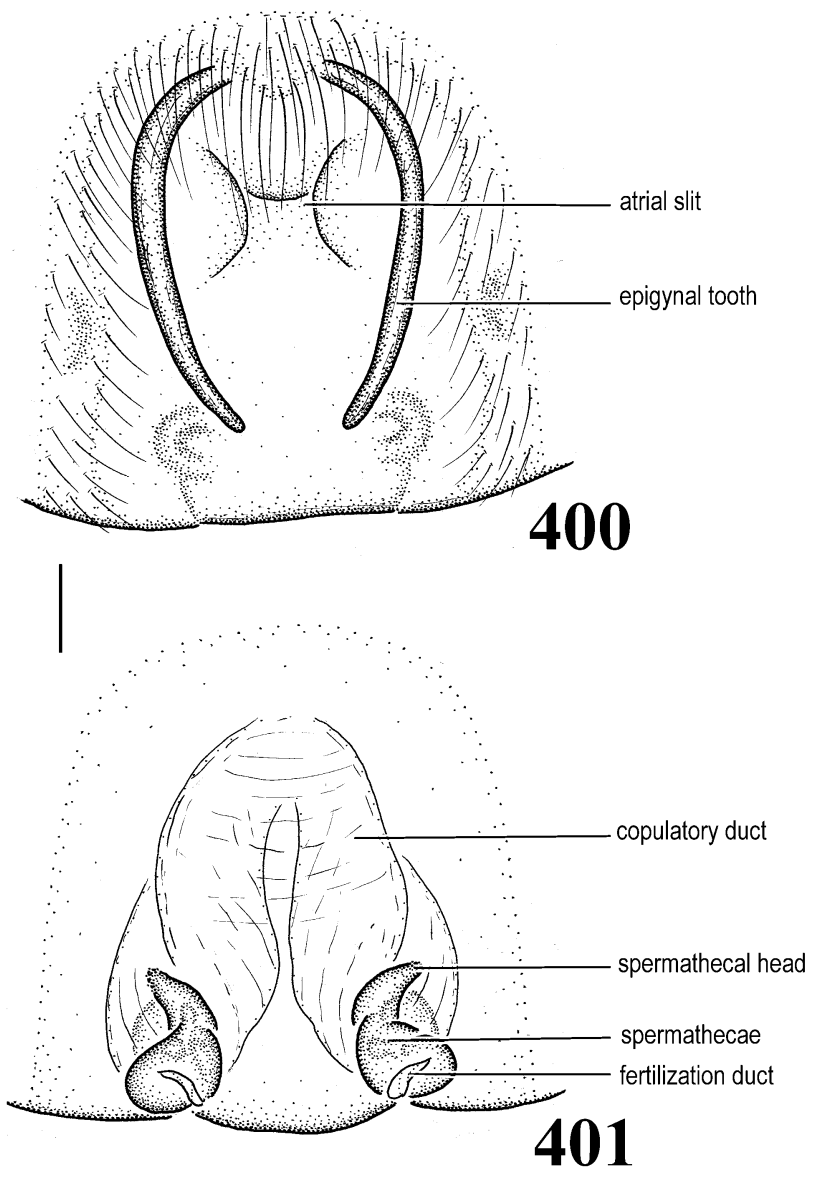

Figs. 400, 401. Urocoras longispinus (Kulczynski), female. 400. Epigynum. 401. Vulva. 




Figs. 402, 403. Urocoras longispinus (Kulczynski), male palp. 402. Ventral view. 403. Retrolateral view.

Epigynum with epigynal teeth absent; atrium reduced to atrial slits; hoods well developed; copulatory ducts short; spermathecal heads small, apparent; spermathecae with stalks long, slender, spiral twice around copulatory ducts, then down close together; spermathecae bases slightly looped (figs. 360, 361). Male palpal patellar apophysis long, with distal end sharply curved dorsally; RTA long, with distal end broad, not extend anteriorly; lateral tibial apophysis present; cymbial furrow elongated, with distal end strongly extended beyond cymbium, dorsal edge strongly concave; conductor long, slender, extended anteriorly; conductor dorsal apophysis absent; conductor lamella small; embolus posterior in origin, long, linear; median apophysis absent (figs. 366-368).

Material Examined: CHINA: Hunan: Changsha, January 7, 1985, 2 male and 2 female paratypes (J. F. Wang, HBI); Changsha, Yuelushan, June 1995, 1 female (X. P. Wang, IZB). Sichuan: Chengdu, Bao-guang Monstery, May 21, 1989, 2 males (P. Beron,
COLL. DEELEMAN-REINHOLD). Jiangsu: Nanjing, Zijin (Purple) Mt., 350-450 m, October 9, 1988, 1 female (P. Beron, COLL. DEELEMAN-REINHOLD). Hubei: Wudangshan, Laoying, May 10, 1982, female holotype and female paratype of Coelotes laoyingensis (HUW).

Distribution: China (Hubei, Hunan, Jiangsu, Sichuan).

\section{TEGECOELOTES OVTCHINNIKOV}

Tegecoelotes Ovtchinnikov, 1999: 68.

TyPE SPECIES: Coelotes bicaudatus Paik, 1976 , by original designation.

DiAGNOSIS: Females can be distinguished by the broad epigynal teeth, the reduced atrium, and the short copulatory ducts (figs. 375, 376), males by the elongated patella and the long, but not spoonlike median apophysis (figs. 377-380).

DESCRIPTION: See description of type species (below). 


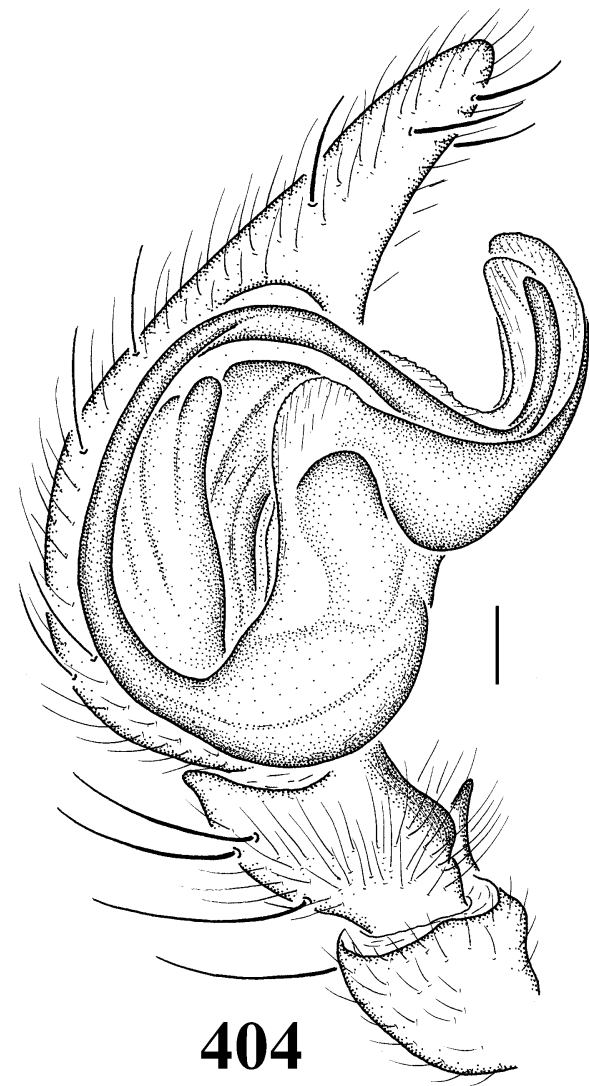

Figs. 404-406. Urocoras longispinus (Kulczynski). 404. Male palp, prolateral view. 405. Carapace, front view. 406. Chelicera, ventral view.

Distribution: China, Japan, Korea, Far eastern Russia (map 17).

COMPOSITION: Five species are included in this genus.

1. Tegecoelotes corasides (Bösenberg and Strand, 1906): male and female types from Saga, Japan, in SMF, examined. NEW COMBINATION (transferred here from Coelotes).

2. Tegecoelotes bicaudatus (Paik, 1976): female holotype from Mt. Ode, Korea, deposited in Kyungpook National University, Taegu, Korea, not examined.

3. Tegecoelotes ignotus (Bösenberg and Strand, 1906): two female types (not further designated) from Japan, in SMF, examined. NEW COMBINATION (transferred here from Coelotes).

4. Tegecoelotes michikoae (Nishikawa, 1977): female holotype, male and female paratypes from Minoo, Osaka Prefecture, Japan,
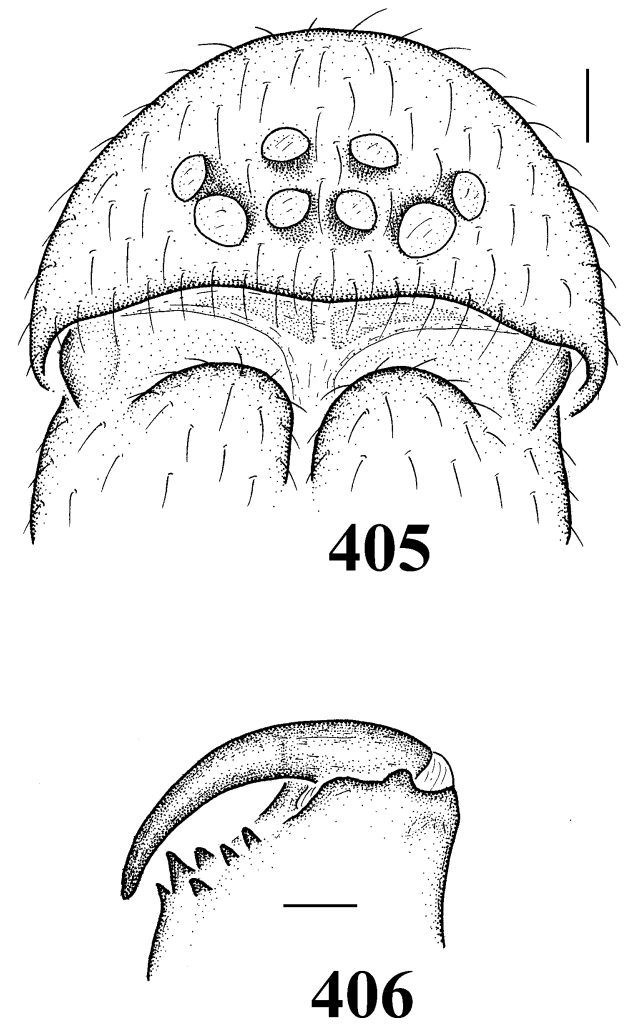

\section{6}

deposited in the Osaka Museum of Natural History, Osaka, and in the Arachnological Society of Japan, Ohtemon-Gakuin University, Osaka, Japan, not examined. NEW COMBINATION (transferred here from Coelotes).

5. Tegecoelotes muscicapa (Bösenberg and Strand, 1906): male holotype from Saga, Japan, in SMF, examined. NEW COMBINATION (valid species, transferred here from Coelotes).

\section{Tegecoelotes bicaudatus (Paik)}

Figures 375-383

Coelotes bicaudatus Paik, 1976: 81, figs. 3, 1315 (female holotype, deposited in Kyungpook National University, Taegu, Korea, not examined). - Paik, 1978: 334, figs. 147.1, 147.2.

Coelotes erraticus Nishikawa, 1983: 125, figs. 16 (male and female types from Mt. Koma-gatake, Tazawako-cho, Senboku-gun, Akita, Ja- 

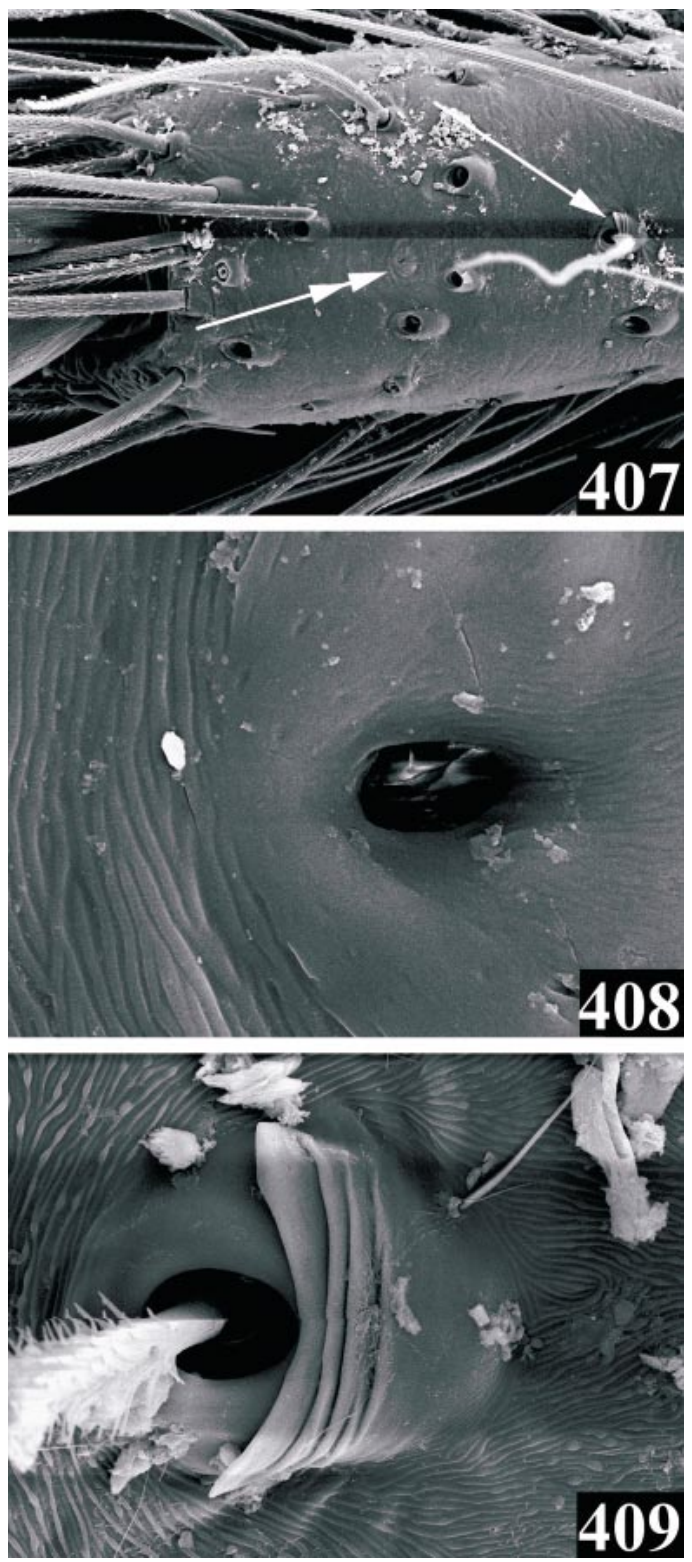

Figs. 407-409. Urocoras longispinus (Kulczynski), female. 407. Tarsus, showing position of tarsal organ (double arrows) and the distalmost trichobothrium (single arrow). 408. Tarsal organ. 409. Trichobothrium.

pan, in NSMT, not examined). - Yaginuma, 1971: 93, figs. 82.1, 82.2;-Matsuda, 1986: 88, figs. 13-16;-Yaginuma, 1986: 151, fig. $80.3-$ - Wang and Zhu, 1991: 5, figs. 13-16; - Song et al., 1999: 375, figs. 217M, 217N, 219G,
219N. First synonymized by Ovtchinnikov, 1999.

Tegecoelotes bicaudatus: Ovtchinnikov, 1999: 68, figs. 16-20. Male first described.

DiAGNOSIS: Females can be distinguished from those of $T$. corasides by the relatively narrow epigynal teeth and the small, anteriorly situated copulatory ducts (figs. 375, 376), males by the short, slender conductor and the spiral median apophysis (figs. 378, 380).

DesCription: Total length 7.00-9.50. From front, anterior eye row slightly procurved, posterior row procurved; eye sizes and arrangements: AME smallest, ALE largest, PLE and PME subequal, slightly smaller than ALE; AME-AME subequal to AME-ALE, ALE-PLE close together, PME-PLE separated by roughly their diameter or slightly longer, PME-PME separated by roughly their diameter or slightly shorter. Clypeal height slightly less than twice AME diameter; chilum divided. Chelicerae with teeth on both margins. Labium as long as wide. Length of female first leg patella + tibia longer than carapace length.

Tarsal organ of this species not examined. Tarsal organ of $T$. corasides on distal end of tarsus, anterior of distalmost trichobothrium. Female spinnerets: ALS with 2 major ampullate gland spigots (MAP), about 46 piriform gland spigots; PMS with 1 or 2 minor ampullate gland spigots (mAP), about 31 aciniform gland spigots, 2 cylindrical gland spigots (CY); PLS not fully expanded in examined specimen, with about 34 aciniform gland spigots, cylindrical gland spigots not seen (figs. 381-383).

Epigynum with broad epigynal teeth, close together; atrium reduced to atrial slit; copulatory ducts short, situated anterad of spermathecae; spermathecal heads short, broad; spermathecae with stalks extended laterally, bases small, situated medially (figs. 375, 376). Male palp with patella strongly elongated; patellar apophysis present; RTA long, with distal end strongly extended; lateral tibial apophysis present; cymbial furrow short; conductor moderately long, with apex close to apex of median apohysis; conductor dorsal apophysis slender; conductor lamella not well developed; embolus prolateral in origin, short, linear; median apophysis not spoon- 




Map 20. Records of Wadotes.

like, long, spiral, with distal end grooved and supported conductor apex; base of median apophysis membranous, slightly expanded (figs. 377-380).

MATERIAl EXAMINED: RUSSIA: Far East: S-Primorie, "Kedrovaya Pad" Reservation in a living house, June 12-December 24, 1977, 2 males and 2 females (B. P. Zakharov, SZM). SOUTH KOREA: Moon-KyangSae-Jae, August 20, 1990, 1 male (J. P. Kim, KAI).

DISTRIBUTION: Korea, far eastern Russia.

\section{TONSILLA WANG AND YIN}

Tonsilla Wang and Yin, 1992: 263. - Platnick, 1997: 671.

TyPE SPECIES: Tonsilla truculenta Wang and Yin, 1992, by original designation.

DiAGNOSIS: Females can be easily recognized by the large epigynal atrium, the posterior extension of the anterior atrial margin, the medially situated epigynal teeth, and the large copulatory ducts (figs. 384, 385), and males by the long, dorsally curved patellar apophysis and the short, lobed conductor (figs. 386-388). Both sexes have three promarginal and two retromarginal cheliceral teeth (fig. 396).

DESCRIPTION: See description of type species (below).

Distribution: Central China (map 18).

COMPOSITION: Four species are included in the genus Tonsilla.

1. Tonsilla eburniformis Wang and Yin, 1992: female holotype from Muyu, Shenlongjia, Hubei, China, in HTU, examined.

2. Tonsilla imitata Wang and Yin, 1992: female holotype from Qiayang, Hunan, China, in HBI, not examined.

3. Tonsilla truculenta Wang and Yin, 1992: female holotype, male and female paratypes from Tianzishan, Sangzhi, Hunan, China, in HEU, examined.

4. Tonsilla variegatus (Wang et al., 1990): female holotype and male paratype from Huangshan, Anhui, China, in HBI, examined. NEW COMBINATION (transferred here from Coelotes). 

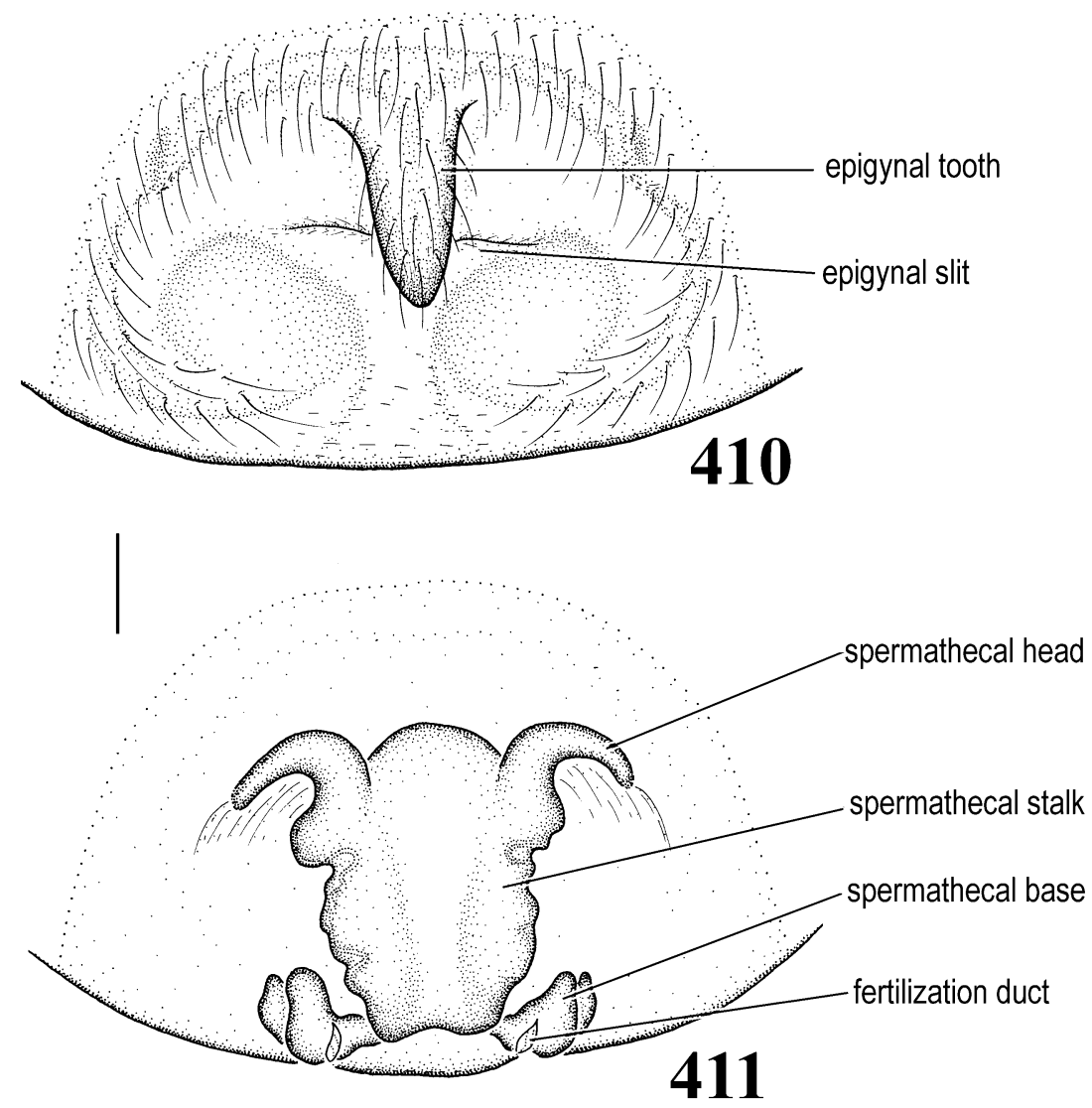

Figs. 410, 411. Wadotes daxiensis Chamberlin, female. 410. Epigynum. 411. Vulva.

\section{Tonsilla truculenta Wang and Yin}

Figures 384-399

Tonsilla truculenta Wang and Yin, 1992: 263, figs. 1-10 (female holotype, male and female paratypes from Tianzishan, Sangzhi, Hunan, China, in HTU, examined). - Song et al., 1999: 395, figs. 13I, 230I-L.

DiAgnosis: Females can be distinguished from those of $T$. eburniformis and T. imitata by the weak, overlapping epigynal teeth (figs. 384, 385). Males can be recognized by the long patellar apophysis and the broad, lobed conductor (figs. 386-399).

DESCRIPTION: Total length 7.00-16.0. From front, anterior eye row slightly procurved, posterior row strongly procurved; AME smallest, ALE subequal to PLE, PME slightly smaller than PLE; AME-AME, AMEALE subequal to AME diameter; ALE-PLE close together; PLE-PME widely separated;
PME-PME subequal to AME-AME, slightly shorter than PME diameter, AME-PME separated by roughly AME diameter or slight longer; clypeus height about twice AME diameter; chilum divided, long (fig. 389). Chelicerae with three promarginal, two retromarginal teeth (fig. 390). Labium longer than wide.

Tarsal organ situated close to distal end of tarsus, anterior of distalmost trichobothrium (figs. 391-393). Apex of ALS with 2 major ampullate gland spigots (MAP), 46-50 piriform gland spigots (PI) in both sexes; PMS with 1 or 2 minor ampullate gland spigots (mAP), about 58-71 aciniform gland spigots (AC) in both sexes, female PMS with 2 cylindrical gland spigots (CY); PLS with about 83-138 aciniform gland spigots in both sexes, 2 cylindrical gland spigots in female (figs. 394-399). 


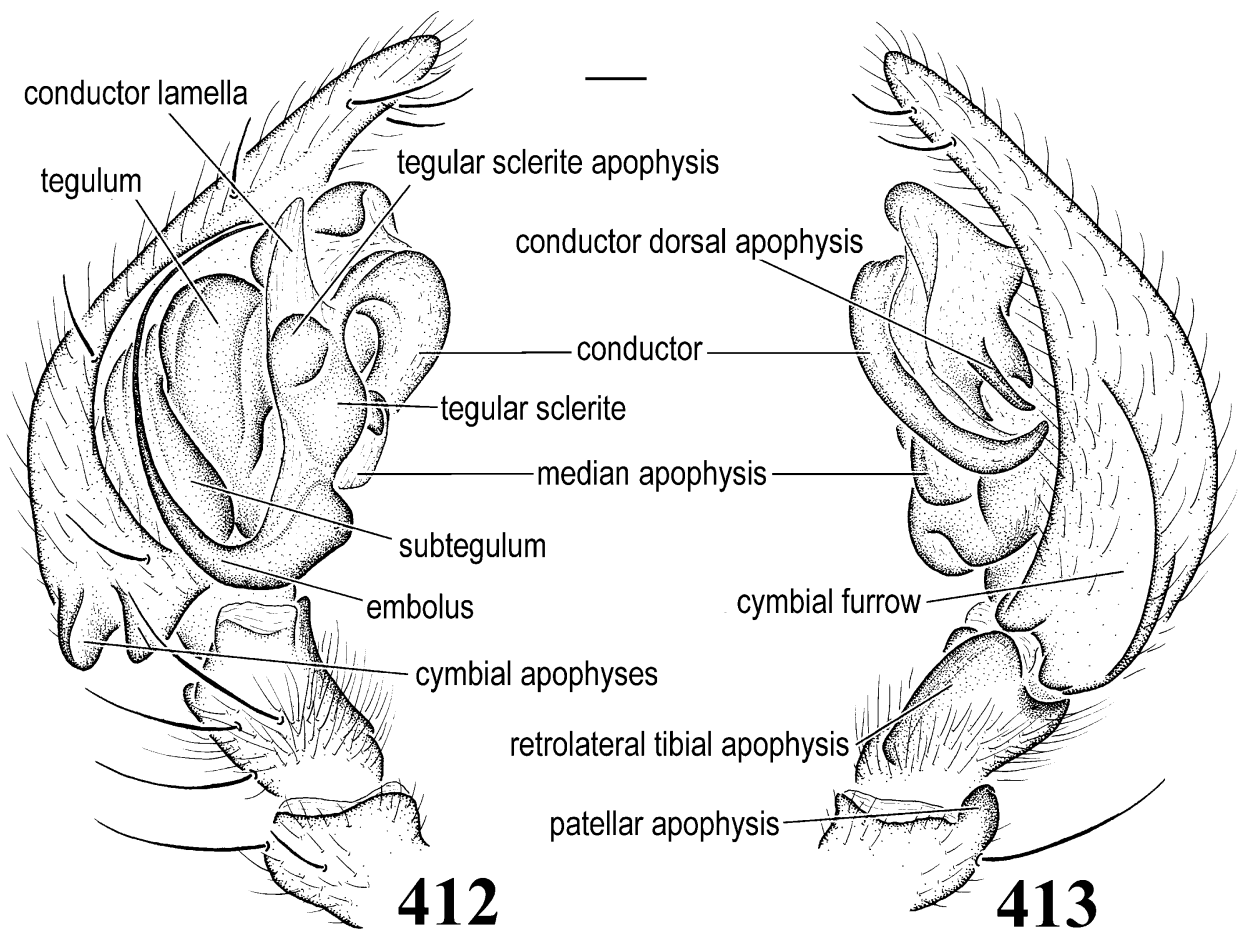

Figs. 412, 413. Wadotes daxiensis Chamberlin, male palp. 412. Prolateral view. 413. Retrolateral view.

Epigynum with slender epigynal teeth, situated close together anteromedially; atrium large; septum present; copulatory ducts broad, situated mesad of spermathecae; spermathecal heads large; spermathecae longitudially elongated, convoluted, with stalks and bases indistinct (figs. 384, 385). Male palp with long, dorsally curved patellar apophysis; RTA long, with distal end strongly extended; lateral tibial apophysis present; cymbial furrow short, with dorsal edge slightly concave; tegular sclerite present; conductor moderately long, lobed; conductor dorsal apophysis slender; conductor lamella short, less developed; embolus basal in origin, moderately long, linear; median apophysis spoonlike, with extended distal end (figs. 386-388).

MAterial ExAmined: CHINA: Hunan: Sangzhi, Tianzishan, October 27, 1989, female holotype, 1 male and 7 female paratypes (J. F. Wang, HTU).

DistRibution: China (Hunan).

\section{UROCORAS OVTCHINNIKOV}

Urocoras Ovtchinnikov, 1999: 68.

TYPE SPECIES: Coelotes longispinus Kulczyn'ski, 1887, by original designation.

DiAGNOSIS: Females can be recognized by the long epigynal teeth, the large, anteriorly extended copulatory ducts, and the small spermathecae (figs. 400, 401), and males by the absence of a conductor dorsal apophysis, the strong embolus, and the smooth, rounded median apophysis (figs. 402-404).

DESCRIPTION: See description of type species (below).

DisTRIBUTION: Eastern Europe (map 19).

COMPOSITION: Five species are included in the genus Urocoras.

1. Urocoras longispinus (Kulczyn'ski, 1897): male lectotype and female paralectotype from Hungary, in MNHN, examined.

2. Urocoras matesianus (de Blauwe, 1973): female holotype and paratype from Roccamandolfi, Matese, Abruzzi, Italy, in MCV, examined. 


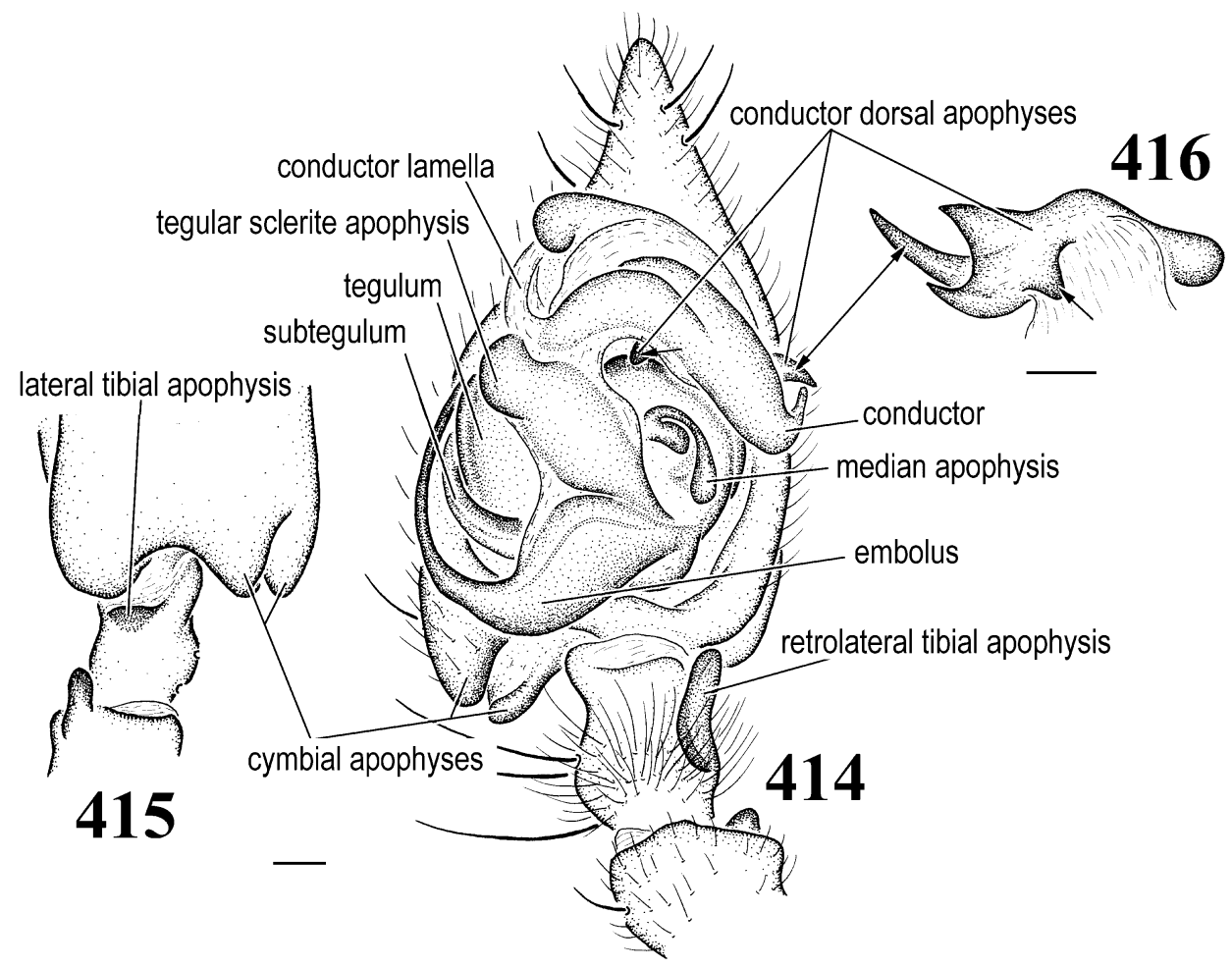

Figs. 414-416. Wadotes daxiensis Chamberlin, male palp. 414. Ventral view. 415. Dorsal view, showing cymbial apophyses. 416. Conductor dorsal apophysis, dorsal view, showing four apophyses.

3. Urocoras munieri (Simon, 1880): male holotype from Sebenino, Yugoslavia, in MNHN, examined. Female specimens from Yugoslavia and Italy examined.

4. Urocoras nicomedis (Brignoli, 1978): female holotype and paratypes from Turkey, in MHNG and MCV, examined. NEW COMBINATION (transferred here from Coelotes).

5. Urocoras phthisicus (Brignoli, 1978): female holotype from Ballidag, Kastamonu, Turkey, in MNHG, examined. NEW COMBINATION (transferred here from Coelotes).

\section{Urocoras longispinus (Kulczyn'ski) Figures 400-409}

Coelotes longispina Kulczyn'ski, 1897: 158, fig. 13 (male lectotype and female paralectotype from Hungary, in MNHN, examined).

Amaurobius longispina: Kulczyn'ski, 1906: 471, figs. 16, 39, 55 .

Coelotes longispina: Drensky, 1942: 40, fig. 5a.Heimer and Nentwig, 1991: 356, fig. 926; Loska, 1969: 107, figs. 73A, 75C, 76A.
Urocoras longispinus: de Blauwe, 1973: 58, figs. 49-53. - Ovtchinnikov, 1999: 68, figs. 21, 22.

Diagnosis: This species can be easily recognized by the strongly elongated, close epigynal teeth and the reduced atrium of females (fig. 400), and by the broad, deeply grooved conductor and the presence of a prolateral tegular sclerite apophysis in males (figs. 402-404).

DESCRIPTION: Total length about 8.009.00. From front, anterior eye row slightly procurved, posterior row procurved; eye sizes and arrangements: ALE largest, AME, PLE, PME subequal, slightly smaller than ALE; AME may be separated by roughly their radius, AME-ALE subequal to AMEAME, ALE-PLE close together, PME-PLE slightly longer than their diameters, PMEPME slightly shorter than PME diameter. Clypeal height slightly longer than AME diameter; chilum divided (fig. 405). Chelicerae with three teeth on both margins (fig. 406). Labium slightly longer than wide. 

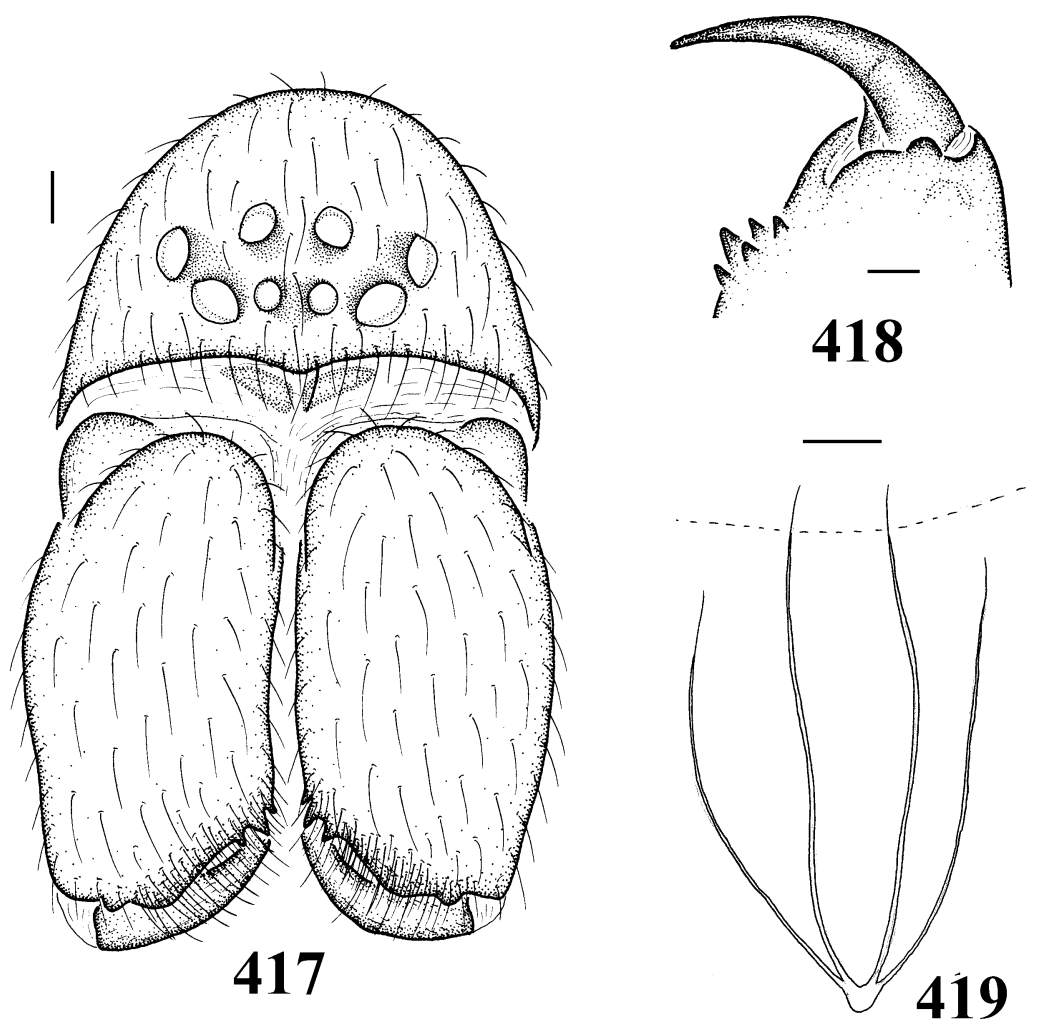

Figs. 417-419. Wadotes daxiensis Chamberlin, female. 417. Carapace, front view. 418. Chelicera, ventral view. 419. Trachea, dashed line refers to position of epigastric furrow.

Tarsal organ situated close to distal end of tarsus, slightly anterior of most distal trichobothrium (figs. 407-409). Spinnerets not examined.

Epigynal teeth strongly elongated, slightly curved; atrium reduced to atrial slit; copulatory ducts large, situated anterad of spermanthecae; spermathecal heads situated anteriorly; spermathecae small, convoluted, with stalks and bases indistinct (figs. 400, 401). Male palp with bifurcated patellar apophysis; RTA long, with distal end slightly extended beyond tibia; lateral tibial apophysis present; cymbial furrow short; tegular sclerite with prolateral apophysis; conductor long, broad; conductor dorsal apophysis absent; conductor lamella moderately developed; embolus prolateral in origin, strong; median apophysis spoonlike, with distal end smooth, not pointed (figs. 402-404).

MATERIAL EXAMINED: BULGARIA: Black Sea, Albena, August 5, 1995, 1 male (V. Po- pov, IZS). HUNGARY: male lectotype and 1 female paralectotype (E. Simon, MNHN, Bocal 2.001, tube $n$ 12.198); 1 female (CAS).

DisTRIBUTION: Bulgaria, Hungary.

\section{WADOTES CHAMBERLIN}

Wadotes Chamberlin, 1925: 120. - Brignoli, 1983: 483; - Platnick, 1989: 426; - Platnick, 1993: 581; - Platnick, 1997: 672.

TyPe SPecies: Wadotes dixiensis Chamberlin, 1925, by original designation.

DiAGNOSIS: Wadotes can be easily recognized by the single, medially situated epigynal tooth of females (fig. 410), and by the presence of proximal cymbial apophyses in males (figs. 412-416). All Wadotes species examined have small AME, and three promarginal and two retromarginal cheliceral teeth (figs. 417, 418). 
DESCRIPTION: See description of type species (below).

Distribution: Eastern North America, from Nova Scotia to Georgia (map 20).

CoMPosition: Twelve species were recognized in a recent revision (Bennett, 1987). One of them, Wadotes primus Fox, 1937, was transferred to the genus Coelotes by Wang et al. (2001) and is placed here as a member of Bifidocoelotes.

1. Wadotes bimucronatus (Simon, 1898).

2. Wadotes calcaratus (Keyserling, 1887).

3. Wadotes carinidactylus Bennett, 1987.

4. Wadotes deceptis Bennett, 1987.

5. Wadotes dixiensis Chamberlin, 1925.

6. Wadotes geogiensis Howell, 1974.

7. Wadotes hybridus (Emerton, 1889).

8. Wadotes mumai Bennett, 1987.

9. Wadotes saturnus Bennett, 1987.

10. Wadotes tennesseensis Gertsch, 1936.

11. Wadotes willsi Bennett, 1987.

\section{Wadotes dixiensis Chamberlin}

Figures 410-428

Wadotes dixiensis Chamberlin, 1925: 120 (male holotype from Lee Co., Alabama, deposited in MCZ, not found).

Wadotes bimucronatus: Muma, 1947: 5, figs. 8, 9, 20, 21, 39, 40 (misidentification).

DiAGNOSIS: Females of this species can be distinguished by the broad spermathecal duct (fig. 411), and males by the shape of conductor dorsal apophysis (figs. 413, 414, 416).

DESCRIPTION: Total length about 5.0012.0. From front, anterior eye row slightly procurved, posterior row procurved; eye sizes and arrangements: AME smallest, about half ALE size, ALE slightly bigger than PLE, PME about 1.5 AME size; AME may be separated by roughly their diameter, AME-ALE about one-half AME diameter and subequal to ALE-PLE, PME-PLE about 1.5 PME diameter, PME-PME slightly shorter than PME diameter, AME-PME about PME diameter. Clypeal height about twice AME diameter, covered with long, strong setae; chilum divided, hairless, short (fig. 417). Chelicerae with three promarginal and two retromarginal teeth (fig. 418). Labium about as long as wide, slightly notched distally. Length of female 1 st leg patella + tibia shorter than carapace length.
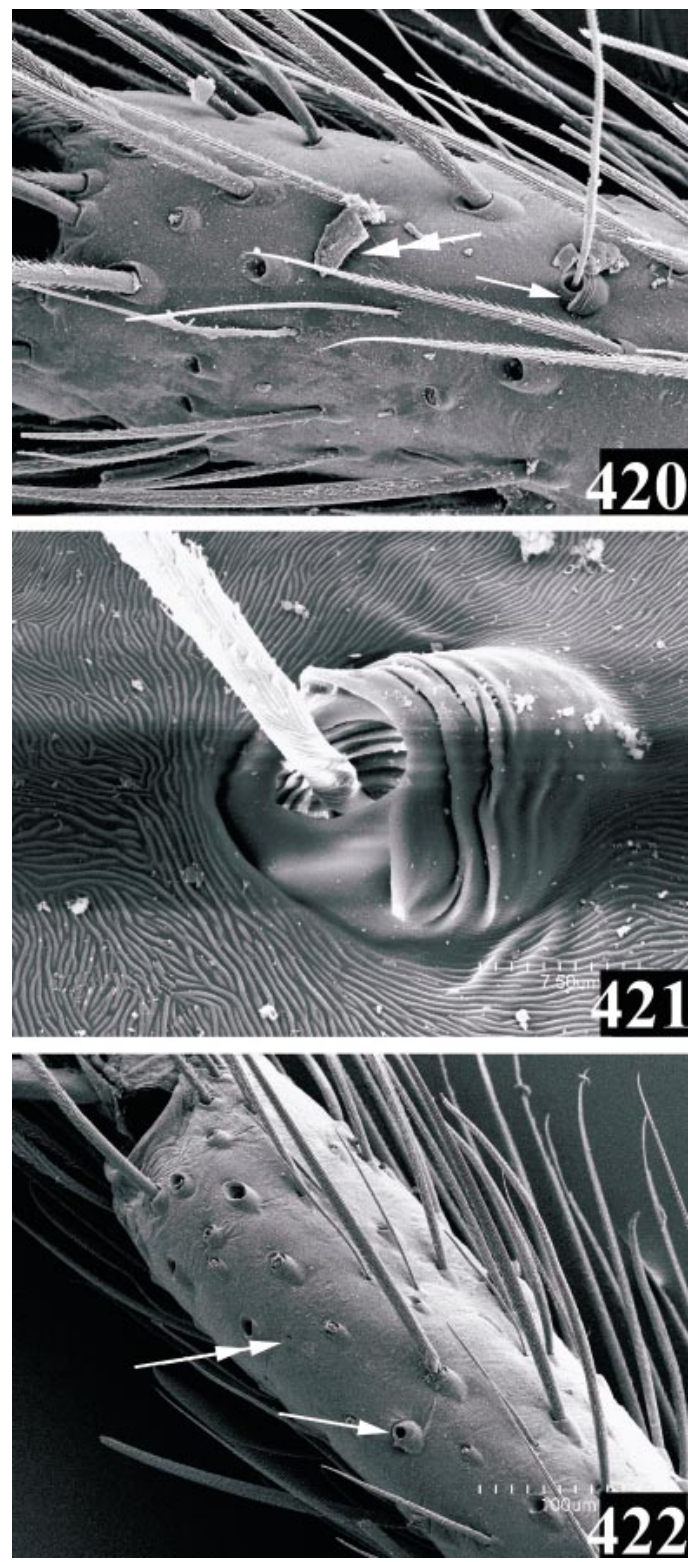

Figs. 420-422. Wadotes daxiensis Chamberlin, female. 420, 422. Tarsus, showing position of tarsal organ (double arrows) and the distalmost trichobothrium (single arrow). 421. Trichobothrium.

Tarsal organ very small, situated close to distal end of tarsus, anterior of distalmost trichobothrium (figs. 420-422). Trachea simple (fig. 419). Apex of ALS with 2 major ampullate gland spigots (MAP), 13-20 piriform gland spigots in both sexes; PMS with 1 or 2 



Figs. 423-425. Wadotes daxiensis Chamberlin, female spinnerets, ventral view, left. 423. ALS. 424. PMS. 425. PLS. Abbreviations are spelled out on p. 6.

minor ampullate gland spigots (mAP) which may be more or less reduced and 37-38 aciniform gland spigots (AC) in both sexes, 2 cylindrical gland spigots (CY) in female; PLS with about 42-48 aciniform gland spigots in both sexes and 2 cylindrical gland spigots in female (figs. 423-428).
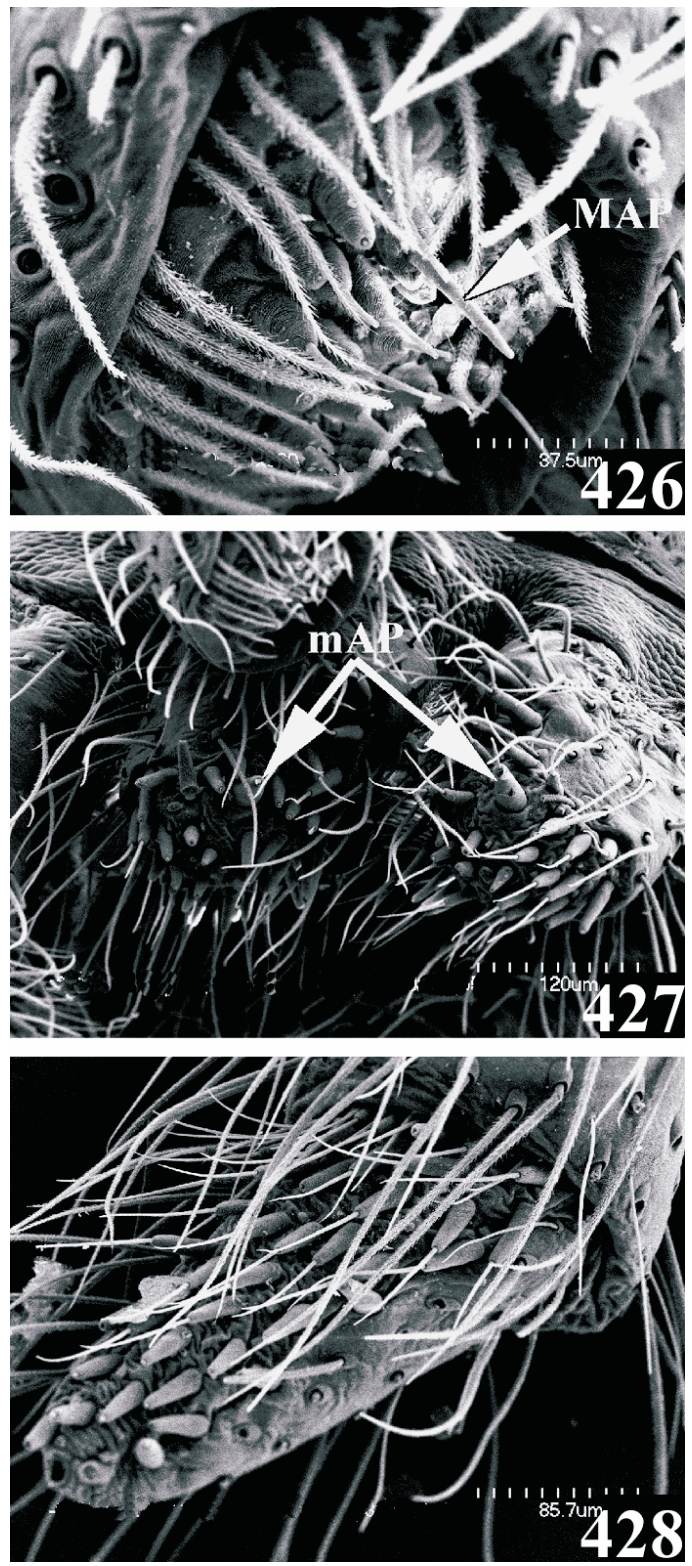

Figs. 426-428. Wadotes daxiensis Chamberlin, male spinnerets, ventral view. 426. ALS, left. 427. PMS. 428. PLS, right. Abbreviations are spelled out on p. 6 .

Female epigynum with single, median, broad epigynal tooth; atrium reduced to atrial slit; copulatory ducts short; spermathecal heads anterolaterally situated; spermathecal stalks long, enclosed in single, broad bursa; spermathecal bases small, bifid, laterally sit- 
uated (figs. 410, 411). Male palp with one patellar apophyses, short; RTA long, occupying most of tibia, but not beyond tibia; lateral tibial apophysis transversely extend, formed small concavity on distal tibia; cymbial furrow moderately long, with distinct dorsal edge; tegular sclerite with prolateral apophysis; conductor long, strong; conductor dorsal apophysis with two dorsal and ventral teeth; conductor lamella large; embolus basal in origin, moderately long, linear; median apophysis spoonlike, with distal end not sharply pointed; tegular carina not apparent (figs. 412-416).

Material Examined: USA: Alabama: Dekalb Co., DeSoto Park, December 1937, 1 male (AMNH); Colbert Co., Wolf Den Cave, September 25, 1940, 1 male (Jones and Archer, AMNH); Walker Co., Johns of Warrior River, October 20, 1912, 1 male (AMNH); Shelby Co., Oak Mt. State Park, May 9, 1947, 1 female (A. F. Archer, AMNH); Colbert Co., Spring Cave, near Maud, September 25, 1940, 1 female (Jones and Archer, AMNH); De Kalb Co., Ravine W. of Mentone, July 27, 1950, 1 female (A. F. Archer, AMNH). Georgia: Ft. Benning, November 20, 1943, 1 male (D. E. Beck, AMNH). North Carolina: Macon Co., Wayali Bald., June 18, 1950, 1 female (A. F. Archer, AMNH); Aquane, W83.39, N32.12, 1 male (AMNH); Highland, April 5, 1929, 1 male (S. C. Biship, AMNH); Coly Co., April 29, 1938, 2 females (J. H. Hubbell, AMNH); Coly Co., April 29, 1938, 1 male and 3 females (J. H. Hubbell, AMNH).

Distribution: North Carolina, Tennessee, Georgia, Alabama.

\section{ACKNOWLEDGMENTS}

This study has been possible only with the help, encouragement, and support of many individuals and institutions.

I thank Jerrold Davis, Norman Platnick, Maurice Tauber, and Quentin Wheeler, the members of my committee at Cornell University, for their continuing encouragement and advice, and for reading and commenting on the manuscript. Helpful suggestions on this research, both in systematics and in other disciplines, during my stay at Cornell University and at the American Museum of Nat- ural History (AMNH) were also provided by Kefyn Catley, Caroline Chaboo, Michael Engel, Bryan Danforth, Rick Hoebeke, Bernhard Huber, Christine Johnson, Zhiwei Liu, Michael Mcdonald, Tam Nguyen, Vladimir Ovtsharenko, Mohammed Shadab, Diana Silva, Louis Sorkin, and Boris Zakcharov.

I offer, deeply, gratitude to the reviewers. Their comments reduced the errors and made the paper more readable and useful.

A fellowship from the American Museum of Natural History supported my study as a graduate student fellow in the joint AMNHCornell University Graduate program. Fieldwork in China was supported by a L.T. Lam Award from the East Asia Program, Cornell University; the American Arachnological Society Research Fund (AAS Fund); the Rawlins Endowment from the Department of Entomology, Cornell University; and the Griswold Fund from the Department of Entomology, Cornell University.

I am especially grateful for materials loaned from the following institutions through the generosity of their curators:

AMNH American Museum of Natural History, New York. N. I. Platnick

AMNH-CU Cornell University Collection loaned to the AMNH. N. I. Platnick

BMNH The Natural History Museum, London. P. Hillyard and J. Margerison

CAS California Academy of Sciences, San Francisco. C. E. Griswold

CNC Canada National Collection of Insects and Arachnids, Ottawa. C.D. Dondale

COLL. DEELEMAN-REINHOLD Collection of C. Deeleman-Reinhold, Netherlands. C. Deeleman-Reinhold

COLL. LEMANN Collection of Zack Lemann, New Orleans, Louisiana. Z. Lemann

COLL. MAURER Collection of R. Maurer, Holderbank, Switzerland. R. Maurer

COLL. REEVES Collection of Will Reeves, Clemson University, Clemson, South Carolina. W. Reeves

COLL. WALTER Collection of J. Walter

CSO Collection of Ovtchinnikov, Bishkek, Kyrghyzstan. S. V. Ovtchinnikov

CUAC Clemson University Arthropod Collection, Clemson, South Carolina. B. Robinson

FSCA Florida State Collection of Arthropods, Gainesville, Florida. G. B. Edwards 
HBI Hunan Biological Institute, Changsha, China. X. J. Peng and C. M. Yin

HEC Hope Entomological Collections, Oxford, England. M. Akinson

HTC Hangzhou Teachers College, Hangzhou, China. Z. F. Chen

HTU Hebei Normal University, Shijiazhuang, China. M. S. Zhu

HUB Hebei University, Baoding, China. M. S. Zhu

HUW Hubei University, Wuhai, China. J. Chen and J. Z. Zhao

IRSNB Institut Royal des Sciences Naturelles de Belgique, Brussels. L. Baert

IZB Institute of Zoology, Beijing. J. Chen and D. X. Song

IZI Institute of Zoology, Innsbruck. K. Thaler

IZS Institute of Zoology, Sofia, Bulgaria. C. D. Deltshev

KAI Korean Arachnological Institute, Seoul. J. P. Kim

MCB Museo de Bergamo, Bergamo, Italy. P. Pantini

MCV Musée Civique d'Histoire Naturelle de Verone, Verona, Italy. R. Salmaso

MCZ Museum of Comparative Zoology, Harvard University, Cambridge, Massachusetts. L. Leibensperger

MEM Mississippi Entomological Museum, Mississippi, Mississippi. R. Brown

MHNG Musée d'Histoire Naturelle, Geneva. B. Hauser

MNHN Musée National d'Histoire Naturelle, Paris. C. Rollard

MZF Museo Zoologico de La Specola, Florence, Italy. S. Whitman

NBUMS Norman Bethune University of Medical Sciences, Changchun, China. J. C. Gao and C. D. Zhu

NHMB Naturhistorisches Museum Basel, Basel. A. Hänggi

NRS Naturhistoriska Riksmuseum, Stockholm. T. Kronestedt

NSMT National Science Museum, Tokyo.

H. Ono

OSU Ohio State University Collection. Cullowhee, North Carolina. F. Coyle

PSU Perm State University, Perm, Russia.

V. Efimik and S. Esyunin

SMF Senckenberg Museum, Frankfurt. M. Grasshoff and J. Martens
SZM Siberian Zoological Museum, Novosibirsk, Russia. D. V. Logunov

THU Department of Biology, Tunghai University, Taichung, Taiwan. I. M. Tso

USNM National Museum of Natural History, Smithsonian Institution, Washington, D.C. J. Coddington

VMNH Virginia Museum of Natural History, Martinsville, Virginia. R. Hoffman

WCU Western Carolina University, Cullowhee, North Carolina. F. Coyle

ZMB Museum fur Naturkunde, Zentralinstitut der Humboldt-Universitut zu Berlin, Berlin. J. Dunlop and Sh. Nawai

ZSM Zoologische Staatssammlung, München. E. Karl

ZII Zoology Institute of Innsbruck, Innsbruck. K. Thaler

\section{REFERENCES}

Banks, N. 1892. The spider fauna of the Upper Cayuga Lake Bassin. Proceedings of the Academy of Natural Sciences of Philadelphia 1892: $11-81$.

Banks, N. 1895. A list of the spiders of Long Island; with descriptions of new species. Journal of the New York Entomological Society 3: 76-93.

Banks, N. 1898. Arachnida from Baja California and other parts of Mexico. Proceedings of the California Academy of Sciences (3) 1: 205308.

Becker, L. 1896. Les Arachnides de Belgique. ( $3^{\circ}$ partie). Annales du Musée royal d'histoire naturelle de Belgique XII: 1-378.

Bennett, R. G. 1987. Systematics and natural history of Wadotes (Araneae, Agelenidae). The Journal of Arachnology 15: 91-128.

Blackwall, J. 1833. Characters of some undescribed genera and species of Araneidae. The London and Edinburgh philosophical magazine and journal of science (3) 3: 104-112, 187-197, 344-352, 436-443.

Blackwall, J. 1834. Researches in zoology. (London) (Araneae, pp. 229-433).

Blackwall, J. 1841. The difference in the number of eyes with which spiders are provided proposed as the basis of their distribution into tribes; with descriptions of newly discovered species and the characters of a new family and three new genera of spiders. The Transactions of the Linnean Society of London 18: 601-670.

Blackwall, J. 1861. A history of the spiders of Great Britain and Ireland. London. 1: 1-174.

Blauwe, R. de. 1973. Révision de la famille des 
Agelenidae (Araneae) de la région méditerranéenne. Bulletin-Institut royal des sciences naturelles de Belgique 49(2): 1-111.

Bösenberg, W. 1902. Die Spinnen Deutschlands. II-IV Zoologica (Stuttgart) 14: 97-384.

Bösenberg, W., and E. Strand. 1906. Japanische Spinnen. Abhandlungen der Senckenbergischen Naturforschenden Gesellschaft 30: 93-422.

Brignoli, P. M. 1976. Spinnen aus Nepal, III. Über einige Spinnen aus dem Himalaya, dazu Revision einiger Arten aus dem Karakorum (Arachnida, Araneae). Ergebn. ForschUnternehmens Nepal Himalaya 5: 229-253.

Brignoli, P. M. 1978a. Ergebnisse der Bhutan-Expedition 1972 des Naturhistorischen Museums in Basel. Araneae: Fam. Oonopidae, Agelenidae, hahniidae und Mimetidae. Entomologica Basiliensia 3: 31-56.

Brignoli, P. M. 1978b. Ragni di Turchia. V. Specie nuove o interessanti, cavernicole ed epigee, di varie famiglie (Araneae). Revue Suisse de Zoologie 85: 461-541.

Brignoli, P. M. 1982. On a few spiders from China (Araneae). Bulletin of the British Arachnological Society 5: 344-351.

Brignoli, P. M. 1983. A catalogue of the Araneae, described between 1940 and 1981. Manchester University Press. 719 pp.

Buchar, J. 1995. Jak je utvárěno teľo pavouka. Zivva 1995(4): 185-187.

Buchar, J., V. Duchác, K. Hûrka, and J. Lallák. 1995. Pavouci-Araneida. In Klíc K Urcování Bezobratlyćh: 104-128. Prague: Scientia.

Cambridge, F. O. P.- 1893. British spiders. British Naturalist 3: 123-170.

Cambridge, O. P.- 1873. On some new species of European spiders. Journal of the Linnean Society, London 11: 530-547.

Cambridge, O. P.- 1885. Araneida. In Scientific results of the second Yarkland mission: 1-115. Calcutta.

Catley, K. M. 1992. Super-cooling and its ecological implications in Coelotes atropos (Araneae, Agelenidae). The Journal of Arachnology 20: 58-63.

Chamberlin, R. V. 1925. Notes on North American spiders heretofore referred to Coelotes. Proceedings of the Biological Society of Washington 38: 119-124.

Charitonov, D. E. 1969. Material'i k faune paukov SSR. Uchenye Zapiski Permskogo Ordena Trudovogo krasnogo Znameni gosudarstvennogo Universiteta Imeni Alekseya Maksimovicha Gorkogo ( $=$ Memories of Sciences of Perm University. Serial: Biological) 179: 59-133.

Chen, J., J. Z. Zhao, and J. F. Wang. 1991. Two new species of the genus Coelotes from Wud- ang Mountain, China. Journal of Hubei University (Natural Sciences) 13(1): 9-12.

Chen, J., and J. Z. Zhao. 1997. Four new species of the genus Coelotes from Hubei, China (Araneae: Amaurobiidae). Acta Arachnologica Sinica 6(2): 87-92.

Chen, Z. F. 1984. Five new species of the genus Coelotes (Agelenidae) from China. Journal of the Hangzhou Normal College (Natural Science) $1: 1-7$.

Chen, Z. F., and Z. H. Zhang. 1991. Fauna of Zhejiang: Araneida. Hangzhou (China): Zhejiang Science and Technology Publishing House, 356 pp.

Chikuni, Y. 1989. Pictorial encyclopedia of spiders in Japan. Tokyo: Kaisei-sha, 310 pp.

Chrysanthus, Fr. 1965. On the identity of Coelotes atropos (Walckenaer), saxatilis (Blackwall) and terrestris (Wider). Tijdschrift voor Entomologie 108(3): 61-71.

Chyzer, C., and W. Kulczyn'ski. 1897. Araneae Hungariae. Budapestini 2: 151-366.

Comstock, J. H. 1910. The palpi of male spiders. Annals of the Entomological Society of America 3: $161-185$.

Comstock, J. H. 1912. The spider book; a manual for the study of the spiders and their near relatives, the scorpions, pseudoscorpions, whipscorpions, harvestmen and other members of the class Arachnida, found in America north of Mexico, with analytical keys for their classification and popular accounts of their habits. Garden City, NY, 721 pp.

Comstock, J. H. 1940. The spider book, revised and edited by W. J. Gertsch. Ithaca, NY: Cornell University Press, 727 pp.

Dahl, M. 1931. Spinnentiere oder Arachnoidea. Agelenidae. In Die Tierwelt Deutschlands: 24: 1-46. Jena.

Deltschev, C. D. 1990. A critical review of genus Coelotes Blackwall in Bulgaria with description of a new species. Acta Zoologica Bulgarica 40: 29-43.

Deltshev, C., and D. Dimitrov. 1996. A new Coelotes, C. brevispinus n. sp. from Bulgarian mountains (Araneae, Agelenidae). Revue Arachnologique 11: 77-79.

Drensky, P. 1915. Arachn. nouv. ou peu conn. de Bulgar. Spisanie na Bulgarskata Academiia na Naukitie 12: 141-176.

Drensky, P. 1939. Über die Identifizierung einiger Spinnenarten, die von Dr. Al. Rosca (1935 u. 1936) als neu für die Bukowina (Rumänien) beschrieben wurden. Izvestya Bulgarica Entomologica Drusestvo 10: 85-87.

Drensky, P. 1942. Die Spinnenfauna Bulgariens, V. Mitteilungen aus den Königl. Naturwissen- 
schaftlichen Instituten in Sofia-Bulgarien 15: $33-60$.

Dufour, L. 1820. Observations générales sur les arachnides et description de quelques espèces nouvelles ou peu connues. Annales générales des sciences physiques. 6: 289-306.

Emerton, I. H. 1889. New England spiders of the families Drassidae, Agelenidae and Dysderidae. Transactions of the Connecticut Academy of Arts and Sciences 8: 166-206.

Emerton, I. H. 1902. The common spiders of the United States. Boston, 225 pp.

Farris, J. S. 1969. A successive approximation approach to character weighting. Systematic Zoology 18: 374-385.

Farris, J. S. 1988. Hennig86, version 1. 5. Computer program distributed by its author. Gering, R. L. 1953. Structure and function of the genitalia in some American agelenid spiders. Smithsonian Miscellaneous Collections 121(4): $1-84$.

Goloboff, P. 1993a. Estimating character weights during tree search. Cladistics 9: 83-91.

Goloboff, P. 1993b. Nona, version 1.8. Computer program distributed by its author. Goloboff, P. 1997. Pee-Wee, version 2.6. Computer program distributed by its author.

Griswold, C. E. 1990. A revision and phylogenetic analysis of the spider subfamily Phyxelidinae (Araneae, Amaurobiidae). Bulletin of the American Museum of Natural History 196: 1206.

Griswold, C. E. 1993. Investigations into the phylogeny of the lycosoid spiders and their kin (Arachnida: Araneae: Lycosoidea). Smithsonian Contributions to Zoology 539: 1-39.

Hansen, H. J. 1882. Spindeldyr. In J. C. Schiödte (editor), Zoologia Danica. 3: 1-81, I-V. Kjöbenhavn.

Heimer, S., and W. Nentwig. 1991. Spinnen Mitteleuropas: ein Bestimmungsbuch. Berlin: Verlag Paul Parey, 543 pp.

Hentz, N. M. 1821. A notice concerning the spider, whose web is used in medicine. Journal of the Academy of Natural Sciences of Philadelphia 2: 53-55.

Hentz, N. M. 1847. Descriptions and figures of the araneides of the United States. Boston Journal of Natural History 5: 443-478.

Hentz, N. M. 1867. Supplement to the descriptions and figures of the araneides of the United States by Nicholas Marcellus Hentz (edited by S.H. Scudder, after death of Hentz). Proceedings of the Boston Society of Natural History 11: $103-111$.

Hu, J. L., and A. H. Li. 1987. The spiders collected from the fields and forests of Xizang Autonomous Region, China. (II). Agricultural In- sects, Spiders, Plant Diseases and Weeds of Xizang 2: 247-353.

Hull, J. E. 1955. British spiders: recent and amended records. Annals and Magazine of Natural History (12) 8: 49-56.

Karsch, E. 1879. Baustoffe zu einer Spinnenfauna von Japan. Verhandlungen des Naturhistorischen Vereines der Preussischen und Westfalens 36: 57-105.

Karsch, E. 1881. Chinesesche Myriapoden und Arachnoiden. Berliner entomologische Zeitschrift 25: 219-220.

Kaston, B. J. 1948. Spiders of Connecticut. Bulletin of Connecticut State Geological and Natural History Survey 70: 1-874.

Keyserling, E. v. 1881. Neue Spinnen aus Amerika. III. Verhandlungen der Zoologisch-Botanischen Gesellschaft in Wien 31: 269-314.

Keyserling, E. v. 1887. Neue Spinnen aus Amerika. VII. Verhandlungen der Zoologisch-Bostanischen Gesellschaft in Wien 37: 421-490.

Koch, L. 1855. Zur Charakteristik des Artenunterschiedes bei den Spinnen im allgemeinen und insbesondere der Gattung Amaurobius. Correspondenz-blatt des Zoologisch-mineralogischen Vereins in Regensburg 9: 158-168.

Koch, L. 1868. Die Arachnidengattungen Amaurobius, Coelotes and Cybaeus. Abhandlungen der Naturhistorischen Gesellschaft zu Nürnberg 4: 1-52.

Koch, L. 1878. Japanesische Arachniden und Myriapoden. Verhandlungen der Zoologisch-Botanischen Gesellschaft in Wien 27: 735-798.

Kroneberg, A. 1875. In A. Fedchenko's Puteshestvie v Turkestan; zoogeographicheskia Izledovania, Arachnida. Nachrichten der k. Gesellschaft der Liebhaber der Naturkunde zu Moskau 19: 1-58.

Kulczyn'ski, V. 1887. Przyczynek do tyrolskiej fauny pajeczakow. Rozprawy I sprawozdania $z$ posiedzen Wydzialu Matematyczno-Przyrodniczego Akademii Umiejetnosci W Krakowie 16: 245-356 (+ Anhang: 1-12).

Kulczyn'ski, V. 1898. Symbola ad faunam aranearum Austriae inferioris cognoscendam. Rozprawy i sprawozdania z posiedzen Wydzialu Matematyczno-Przyrodniczego Akademii Umiejetnosci W Krakowie 36: 1-114.

Kulczyn'ski, V. 1906. Fragmenta arachnologica. VII. Bulletin international de l'Academie des Sciences de Cracovie 1906: 417-476.

Kulczyn'ski, V. 1908. Araneae musei nationalis Hungarici in regionibus Indica et Australia a Ludovico Biro collectae. Annales historico-naturales Musei Nationalis Hungarici 6: 428-494.

Leech, R. E. A revision of the Nearctic Amaurobiidae (Arachnida: Araneida). Memories of Entomological Society of Canada 84: 1-182. 
Lehtinen, P. T. 1967. Classification of the cribellate spiders and some allied families, with notes on the evolution of the suborder Araneomorpha. Annales Zoologici Fennici 4: 199-468.

Levi, H. W., and O. Kraus. 1964. Amaurobius C.L. Koch, 1837 and Coelotes. Bulletin of Zoological Nomenclature 21(2): 150-153.

Locket, G. H., and A. F. Millidge. 1953. British spiders II. London: Ray Society, 449 pp.

Locket, G. H., A. F. Millidge, and P. Merrett. 1974. British Spiders III. London: Ray Society, $315 \mathrm{pp}$.

Loksa, I. 1969. Araneae I. Fauna Hungariae 97: $1-133$.

Marusik, Yu. M., and D. V. Logunov. 1991. Spiders of the superfamily Amaurobiidae from Sakhalin and Kurily Islands. Zoologicheskii Zhurnal 70(9): 87-91.

Matsuda, M. 1986. Supplementary note to "A list of spiders of the central mountain district (Taisetsuzan National Park), Hokkaido", Bulletin of the Higashi Taisetsu Museum of Natural History 8: 83-92.

Maurer, R. 1982. Zur Kenntnis der Gattung Coelotes (Araneae, Agelenidae) in Alpenländern II. Die pastor-Gruppe der italienischen und französischen Alpen-Beschreibung von C. pastor lessinensis ssp. n. Bollettino del Museo Civico di Storia Naturale di Verona 8: 165-183.

Miller, F. 1971. Pavouci-Araneida. Klic Zvireny CSSR 4: 51-306.

Muma, M. H. 1943. Common spiders of Maryland. The Natural History Society of Maryland 8: $1-179$.

Muma, M. H. 1946. North American Agelenidae of the genus Coras Simon. American Museum Novitates 1329: 1-20.

Muma, M. H. 1947. North American Agelenidae of the genus Wadotes Chamberlin. American Museum Novitates 1334: 112.

Nishikawa, Y. 1974. Japanese spiders of the genus Coelotes. Faculty of Letters, Rev. Otemon-Gakuin University 8: 174-182.

Nishikawa, Y. 1975. Preliminary notes on the geographical distribution and variation of $\mathrm{Coe}$ lotes exitialis (Araneae: Agelenidae). Faculty of Letters, Rev. Otemon-Gakuin University 9: 175-187.

Nishikawa, Y. 1983. Spiders of the genus Coelotes (Araneae, Agelenidae) from the mountains of the Tohoku District, northern Japan. Memoirs. National Science Museum, Tokyo 16: 123-136.

Nishikawa, Y. 1995. A new ground-living spider of the genus Coelotes (Araneae, Agelenidae) from Northern Vietnam. Special Bulletin of the Japanese Society of Coleopterology 4: 139142.
Ovtchinnikov, S. V. 1988. Materials on spider fauna of the superfamily Amaurobioidea of Kirghizia. Entomologicheskie issledovaniia v Kirgizii 19: 139-152.

Ovtchinnikov, S. V. 1999. On the supraspecific systematics of the subfamily Coelotinae (Araneae, Amaurobiidae) in the former USSR fauna. TETHYS Entomological Research 1: 6380.

Ovtsharenko, V. I., and V. Y. Fet. 1980. Fauna and ecology of spiders (Aranei) of Badhyz (Turkmenian SSR). Revue d'Entomologie de l'URSS. 59: 442-447.

Paik, K. Y. 1971. Korea spiders of genus Coras. Korean Journal of Zoology 14: 7-18.

Paik, K. Y. 1976. Five new spiders of genus Coelotes (Araneae: Agelenidae). Educational Journal of the Kyorbuk Taehakkyo Teachers College 18: 77-88.

Paik, K. Y. 1978. Araneae. Illustration flora and fauna of Korea 21: 1-548.

Paik, K. Y., T. Yaginuma, and J. Namkung 1969. Results of the speleological survey in South Korea 1966. 19. Cave dwelling spiders from the southern part of Korea. Bulletin of the National Science Museum 12: 795-844.

Peng, X. J., and J. F. Wang. 1997. Seven new species of the genus Coelotes (Araneae, Agelenidae) from China. Bulletin of the British Arachnological Society 10(9): 327-333.

Peng, X. J., H. M. Yan, M. X. Liu, and J. P. Kim. 1998. Two new species of the genus Coelotes (Araneae: Agelenidae) from China. Korean Arachnology 14(1): 77-80.

Petto, R. 1990. Abundance and prey of Coelotes terrestris (Wider) (Araneae, Agelenidae) in hedges. Bulletin of the British Arachnological Society 8(6): 185-193.

Platnick, N. I. 1989. Advances in spider taxonomy 1981-1987, a supplement to Brignoli's “A catalogue of the Araneae described between 1940 and 1981. "Manchester: Manchester University Press, 673 pp.

Platnick, N. I. 1993. Advances in spider taxonomy 1988-1991, with synonymies and transfers 1940-1980. New York: New York Entomological Society in association with the American Museum of Natural History, 846 pp.

Platnick, N. I. 1997. Advances in spider taxonomy 1992-1995, with redescriptions 1940-1980. New York: New York Entomological Society in association with the American Museum of Natural History, 976 pp.

Reimoser, E. 1931. Einheimische Spinnen. 6-9. Die Naturwissenschaften (Wien) 7(2): 37-41.

Roberts, M. J. 1985. The spiders of Great Britain and Ireland, Vol. I: Atypidae to Theridiosomatidae. Colchester, England: Harley Books. 
Roberts, M. J. 1995. Collins field guide: spiders of Britain and Northern Europe. London: HarperCollins, 383 pp.

Roewer, C. 1954. Katalog der Araneae 1. Bremen.

Roth, V. D. 1982. Handbook for spider identification. Portal, AZ: Published by the author.

Roth, V. D. 1985. Spider genera of North America. Gainesville, FL: American Arachnological Society.

Roth, V. D. 1994. Spider genera of North America, with keys to families and genera, and a guide to literature, 3rd ed. Gainesville, FL: American Arachnological Society, 203 pp.

Roth, V. D., and P. L. Brame. 1972. Nearctic genera of the spider family Agelenidae. American Museum Novitates 2505: 1-52.

Schenkel, E. 1923. Beitrag zur Spinnenkunde. Beiträge zur Kenntnis der Wirbellosen terrestrischen Nivalfauna der schweizerischen Hochgebirge. Verhandlungen der Naturforschenden Gesellschaft in Basel 34: 78-127.

Schenkel, E. 1925. Beitrag zur Kenntnis der Spinnenfauna von Todtmoos. Mitteilungen des Badischen Landesvereins für Naturkunde und Naturschutz e.V., Freiburg i. 1: 347-353.

Schenkel, E. 1963. Ostasiatische Spinnen aus dem Muséum d'Histoire naturelle de Paris. Mémoires du Muséum National d'Histoire Naturelle (A, Zool.) 25: 1-481.

Simon, E. 1864. Histoire naturelle des araignées (aranéides). Paris, $540 \mathrm{pp}$.

Simon, E. 1870. Aranéides noveaux ou peu connus du midi de l'Europe. Sulle specie europee della famiglia degli Attidi. Mémoires de la Société royale des sciences de Liège (2) 3: 271358.

Simon, E. 1875. Les Arachnides de France. 2: 350 pp. Paris.

Simon, E. 1876. Etudes arachnologiques. 4e mémoire. VIII. Description d'un Coelotes noveau pour la faune française. Annales de la Société Entomologique de France (5) 6: 92.

Simon, E. 1880a. Etudes arachnologiques. 11e Mémoire. XVII. Arachnides recueilles aux environs de Pékin par M. V. Collin de Plancy. Annales de la Société Entomologique de France (5) 10: 97-128.

Simon, E. 1880b. Arachnides recueilles á Sebenico (Dalmatie), par M. Munier-Chalmas. Annales de la Société Entomologique de France (5) 10(Bull.): 35-36.

Simon, E. 1891. Descr. nov. spec., in: Gasperini, R. Prilog fauni dalmatinskih pauka (Araneae et Opiliones). God. izv. vel. Realke Splitu: 31-45.

Simon, E. 1898. Histoire naturelle des araignées. 2: 193-380. Paris.

Simon, E. 1937. Les arachnides de France. Tome VI. Synopsis générale et catalogue des espèces françaises de 1'ordre des Araneae; 5 et derniére partie 6: 979-1298. Paris.

Song, D. X., M. S. Zhu, S. S. Gao, and J. D. Guan. 1993. On new species of the genera Coelotes and Tegenaria from Liaoning, China (Araneae: Agelenidae). Sinozoologia 10: 93-98.

Song, D. X., M. S. Zhu, and J. Chen. 1999. The spiders of China. Shijiazhuang, China: Hebei Science and Technology Publishing House, 640 pp.

Spassky, S. 1939. Araneae palaearcticae novae III.-Festschrift zum 60. Geburtstage von professor dr. Embrik Strand 5: 138-144.

Steinberger, K. H. 1989. Ein Beitrag zur epigaischen Spinnenfauna Karntens (Arachnida: Aranei). Carinthia II 179: 603-609.

Tretzel, E. 1961. Biologie, okologie und brutpelege von Coelotes terrestris (Wider) (Araneae, Agelenidae). Teil I: Biologie und okologie. Zeitschrift für Morphologie der Tiere 49: 658745.

Tyschchenko, V. P. 1971. Opredelitel' paukov evropejskoj casti SSSR. Leningrad, $281 \mathrm{pp}$.

Uyemura, T. 1936. On a spider, Coelotes exitialis, from Mt. Ohyama, Kanagawaken, Japan. Acta Arachnologica 1: 10-12.

Uyemura, T. 1937. How to discriminate between two related species (1). Acta Arachnologica 2: 55-64.

Walckenaer, C. A. de. 1830. Aranéides. In Faune française ou histoire naturelle générale et particulière des animaux qui se trouvent en France, constamment ou passagèrement, à la surface du sol, dans les eaux qui le baignent et dans le littoral des mers qui le bornent par Viellot, Desmarrey, Ducrotoy, Audinet, Lepelletier et Walckenaer. livr. 26: 97-175, livr. 29: 177-240. Paris.

Walckenaer, C. A. de. 1837. Histoire naturelle des Insectes. Aptères. 1: 682 pp. Paris.

Walckenaer, C. A. de. 1842. Histoire naturelle des Insectes. Aptères. 2: 549 pp. Paris.

Walckenaer, C. A. de. 1847. Histoire naturelles des Insectes. Aptères. 4: 623 pp. (Araneae, pp. 365-564). Paris.

Wang, J. F. 1994. Descriptions of a new genus and two new species of Amaurobiidae from China (Araneae). Acta Zootaxonomica Sinica 19: 281-285.

Wang, J. F., C. M. Yin, X. J. Peng, and L. P. Xie. 1990. New species of the spiders of the genus Coelotes from China (Araneae: Agelenidae). In Spiders in China: One Hundred New and Newly Recorded Species of the Families Araneidae and Agelenidae: 172-253. Changsha, China: Hunan Normal University Press.

Wang, J. F., X. J. Peng, and J. P. Kim. 1996. Two new species of the genus Coras (Araneae, Age- 
lenidae) from China. Korean Arachnology 12(2): 77-81.

Wang, J. F., and C. D. Zhu. 1991. Four new species and a new record of the genus Coelotes from China (Araneae: Agelenidae). Sichuan Journal of Zoology 10(4): 3-6.

Wang, J. F., and C. M. Yin. 1992. A new genus and three new species of funnel-web spiders from south China (Araneae: Agelenidae). Journal of the Hunan Normal University (Natural Science) 15: 263-272.

Wang, X. P., and H. Ono. 1998. The Coelotine Spiders (Araneae, Amaurobiidae) of Taiwan. Bulletin of the National Science Museum, Series A (Zoology) 24(3): 141-159.

Wang, X. P., J. C. Ran, and H. M. Chen. 1999. A new species of Mallinella from China (Araneae, Zodariidae). Bulletin of the British Arachnological Society 11: 193-194.

Wang, X. P., I. Tso, and H. Y. Wu. 2001. Three new Coelotes Spiders (Araneae, Amaurobiidae) from Taiwan. Zoological Studies 40(2): 127133.

Wiehle, H. 1963. Beiträge zur Kenntnis der deutschen Spinnenfauna III. Zoologische Jahrbücher 90: 227-298.

Wiehle, H. 1964. Spinnen aus Solvenien, II. Senckenbergiana Biologica 45: 641-652.

Wunderlich, J. 1986. Spinnenfauna gestern und heute: Fossile Spinnen in Bernstein und ihre heute lebenden Verwandten. Wiesbaden: Quelle und Meyer.

Yaginuma, T. 1960. Spiders of Japan in color. Osaka: Hoikusha, 186 pp.

Yaginuma, T. 1971. Spiders of Japan in color (enlarged and revised edition). Osaka: Hoikusha, vi +197 pp.

Yaginuma, T. 1973. Males of Coelotes exitialis and $C$. antri. Atypus 61: 25-26.

Yaginuma, T. 1986. Spiders of Japan in color (new ed.). Osaka: Hoikusha Publishing Co.

Zhang, Y. J., X. J. Peng, and J. P. Kim. 1998. Five new species of the genus Coelotes (Araneae: Agelenidae) from China. The Korean Journal of Systematic Zoology 13(4): 291-296.

Zhu, C. D. 1983. A list of Chinese spiders (revised in 1983). Journal of Norman Bethune University of Medical Sciences 9(suppl.): 1130.

\section{APPENDIX}

\section{List of Other Coelotes Species}

\section{From China}

1. Coelotes acidentatus Peng and Yin, 1998

2. C. adligansus Peng and Yin, 1998

3. C. amplilamnis Saito, 1936

4. C. amygdaliformis Zhu and Wang, 1991

5. C. argenteus Wang et al., 1990

6. C. bicultratus Chen, Zhao and Wang, 1991

7. C. bituberculatus Wang et al., 1990

8. C. brachiatus Wang et al., 1990

9. C. brunneus $\mathrm{Hu}$ and $\mathrm{Li}, 1987$

10. C. carinatus Wang et al., 1990

11. C. chaiqiaoensis Zhang, Peng and Kim, 1998

12. C. cylistus Peng and Wang, 1997

13. C. denisi Schenkel, 1963

14. C. digitusiformis Wang et al., 1990

15. C. funiushanensis $\mathrm{Hu}$, Wang and Wang, 1991

16. C. galeiformis Wang et al., 1990

17. C. guttatus Wang et al., 1990

18. C. gypsarpageus Zhu and Wang, 1991

19. C. gyriniformis Wang and Zhu, 1991

20. C. hangzhouensis Chen, 1984

21. C. huizhunesis Wang and Xie, 1988

22. C. jiangyongensis Peng, Gong and Kim, 1996

23. C. jucundus Chen, 1997

24. C. kulianganus Chamberlin, 1924
25. C. lyratus Wang et al., 1990

26. C. maculatus Zhang, Peng and Kim, 1998

27. C. magniceps Schenkel, 1936

28. C. magnidentatus Schenkel, 1963

29. C. mastrucatus Wang et al., 1990

30. C. microps Schenkel, 1963

31. C. modestus Simon, 1880

32. C. nanyuensis Peng and Yin, 1998

33. C. nariceus Zhu and Wang, 1994

34. C. ningmingensis Peng et al., 1998

35. C. noctulus Wang et al., 1990

36. C. ornatus Wang et al., 1990

37. C. palinitropus Zhu and Wang, 1994

38. C. penicillatus Wang et al., 1990

39. C. pervicax $\mathrm{Hu}$ and $\mathrm{Li}, 1987$

40. C. plancyi Simon, 1880

41. C. prolixus Wang et al., 1990

42. C. quadratus Wang et al., 1990

43. C. robustus Wang et al., 1990

44. C. rufuloides Zhang, Peng and Kim, 1997

45. C. rufulus Wang et al., 1990

46. C. saccatus Peng and Yin, 1998

47. C. septus Wang et al., 1990

48. C. shuangpaiensis Peng, Gong and Kim, 1996

49. C. streptus Zhu and Wang, 1994

50. C. strophadatus Zhu and Wang, 1991

51. C. syzygiatus Zhu and Wang, 1994

52. C. tautispinus Wang et al., 1990 
53. C. terebratus Peng and Wang, 1997

54. C. tiantongensis Zhang, Peng and Kim, 1998

55. C. triatus Zhu and Wang, 1994

56. C. triglochinatus Zhu and Wang, 1991

57. C. tryblionatus Wang and Zhu, 1991

58. C. uncinatus Wang et al., 1990

59. C. undulatus $\mathrm{Hu}$ and Wang, 1990

60. C. wangi Chen and Zhao, 1997

61. C. yadongensis ( $\mathrm{Hu}$ and $\mathrm{Li}, 1987)$

From Japan

1. Coelotes amamiensis Shimojana, 1989

2. C. cavicola (Komatsu, 1961)

3. C. decolor Nishikawa, 1973

4. C. hamamurai Yaginuma, 1967

5. C. hexommatus (Komatsu, 1957)

6. C. inabaensis Arita, 1974

7. C. kintaroi Nishikawa, 1983

8. C. kumensis Shimojana, 1989
9. C. obako Nishikawa, 1983

10. C. okinawensis Shimojana, 1989

11. C. personatus Nishikawa, 1973

12. C. tarumii Arita, 1976

13. C. tumidivulvus Nishikawa, 1980

14. C. uenoi Yamaguchi and Yaginuma, 1971

15. C. unicatus Yaginuma, 1977

From Europe

1. C. luculli Brignoli, 1978

2. C. obesus Simon, 1875

3. C. rhododendri Brignoli, 1978

4. C. simoni Strand, 1907

5. C. titaniacus Brignoli, 1977

6. C. vallei Brignoli, 1977

From Himalayas and India

1. C. simplex O. P.-Cambridge, 1885

2. C. stylifer Caporiacco, 1935

3. C. tegenarioides O. P.-Cambridge, 1885 